\title{
A PRECISE MEASUREMENT OF THE LEFT-RIGHT ASYMMETRY OF $Z$ BOSON PRODUCTION AT THE SLAC LINEAR COLLIDER
}

\author{
Ross C. King \\ Stanford Linear Accelerator Center \\ Stanford University, Stanford, CA 94309
}

September 1994

Prepared for the Department of Energy under contract number DE-AC03-76SF00515

Printed in the United States of America. Available from the National Technical Information Service, U.S. Department of Commerce, 5285 Port Royal Road, Springfield, Virginia 22161.

*Ph.D. thesis. 


\section{Abstract}

We present a precise measurement of the left-right cross section asymmetry of $Z$ boson production $\left(A_{L R}\right)$ observed in 1993 data at the SLAC linear collider. The $A_{L R}$ experiment provides a direct measure of the effective weak mixing angle through the initial state couplings of the electron to the $Z$. During the 1993 run of the SLC, the SLD detector recorded $49,392 \mathrm{Z}$ events produced by the collision of longitudinally polarized electrons on unpolarized positrons at a center-of-mass energy of $91.26 \mathrm{GeV}$. A Compton polarimeter measured the luminosity-weighted electron polarization to be $(63.4 \pm 1.3) \% . A_{L R}$ was measured to be $0.1617 \pm 0.0071$ (stat.) \pm 0.0033 (syst.), which determines the effective weak mixing angle to be $\sin ^{2} \theta_{W}^{\text {eff }}=0.2292 \pm 0.0009$ (stat.) \pm 0.0004 (syst.). This measurement of $A_{L R}$ is incompatible at the level of two standard deviations with the value predicted by a fit of several other electroweak measurements to the Standard Model. 


\section{DISCLAIMER}

Portions of this document may be illegible in electronic image products. Images are produced from the best available original document. 


\section{Acknowledgments}

First thanks go to my adviser, Morris Swartz, for knowing everything about particle physics and statistics as well, and for always having the time and patience to teach me.

Thanks also to:

Peter Rowson, Mike Woods, Mike Fero, Dave Calloway, and Bruce Schumm, for providing further instruction and camaraderie, as well as for creating and maintaining the spirit of the Compton Cowboy.

My fellow graduate students, Amit Lath and Tom Junk, for sharing their knowledge and skills during our tenure together. Special thanks to Kevin Pitts, John Yamartino, Ram BenDavid, and Rob Elia, for paving the way.

David Williams, for tips on PAW, and Suzanne Williams, for help with FRAMEMAKER, publications plots, and for the food.

And finally, thanks to my wife, Karin, for providing me with the incentive to reach my primary goal: being able to bench-press my own weight. Thanks also for the incentive necessary to finish this document. :) 


\section{Table of Contents}

Abstract

Acknowledgments $\quad$ iii

List of Tables _ $1 x$

List of Figures $\quad x$

Chapter 1: Physics Background and Motivation 1

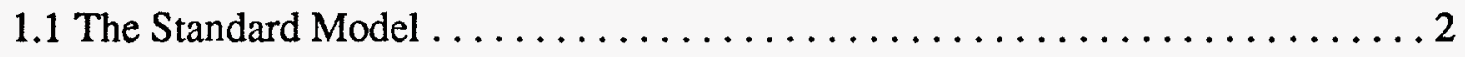

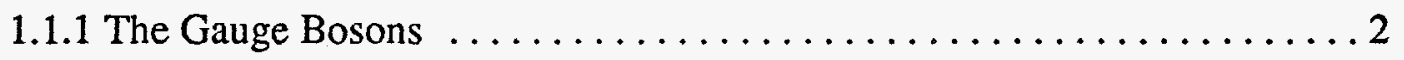

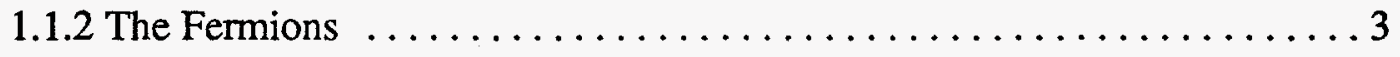

1.1.3 Spontaneous Symmetry Breaking $\ldots \ldots \ldots \ldots \ldots \ldots \ldots \ldots$

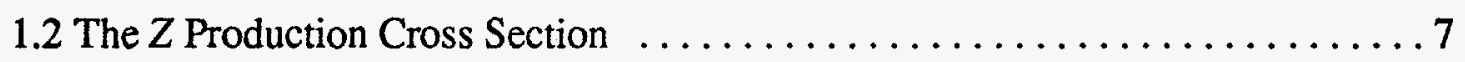

1.2.1 Defining Polarization $\ldots \ldots \ldots \ldots \ldots \ldots \ldots \ldots \ldots \ldots$

1.2.2 The Photon Exchange $\ldots \ldots \ldots \ldots \ldots \ldots \ldots \ldots \ldots \ldots \ldots \ldots$

1.2.3 The $Z$ Exchange $\ldots \ldots \ldots \ldots \ldots \ldots \ldots \ldots \ldots \ldots \ldots \ldots \ldots \ldots \ldots \ldots \ldots \ldots \ldots \ldots$

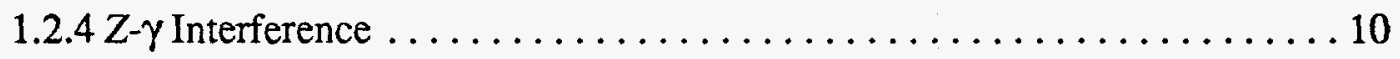

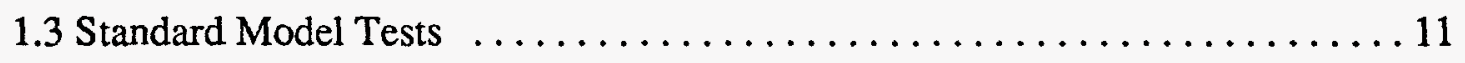

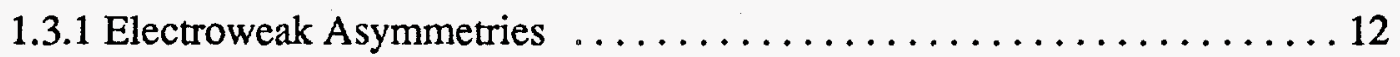

1.3.2 Width of the $Z$ Resonance . . . . . . . . . . . . . . 14

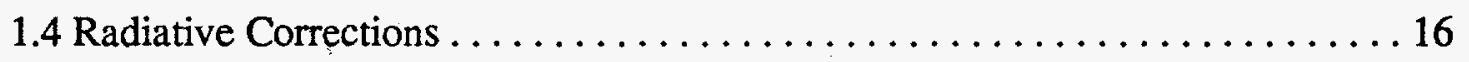




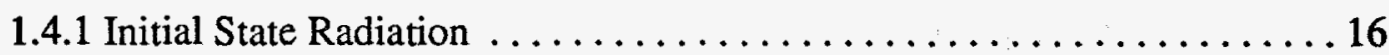

1.4.2 Oblique Corrections $\ldots \ldots \ldots \ldots \ldots \ldots \ldots \ldots \ldots \ldots \ldots \ldots \ldots \ldots$

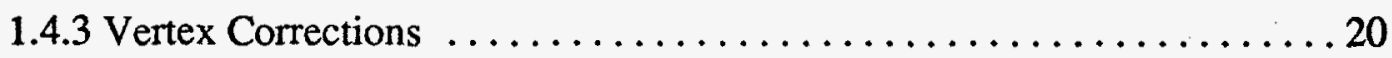

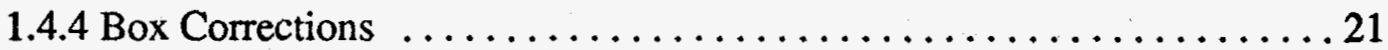

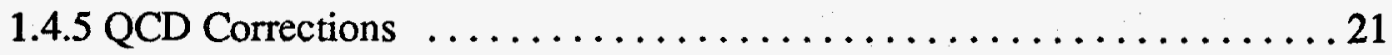

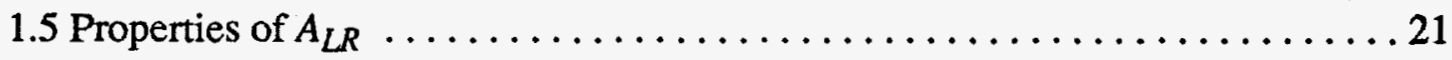

1.5.1 The Measured Left-Right Asymmetry $\ldots \ldots \ldots \ldots \ldots \ldots \ldots \ldots .22$

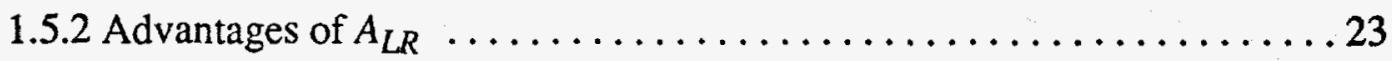

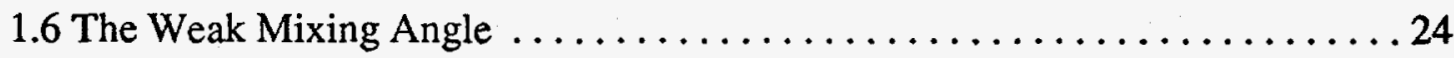

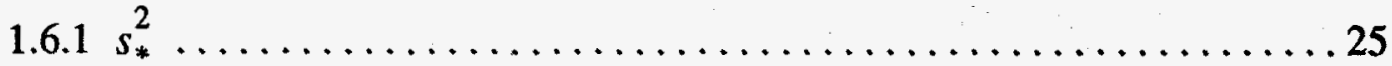

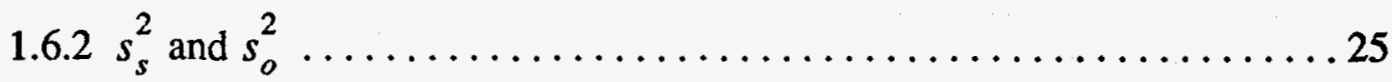

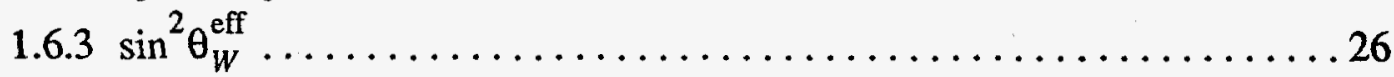

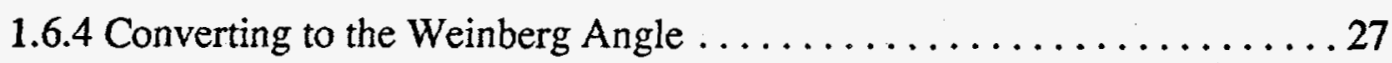

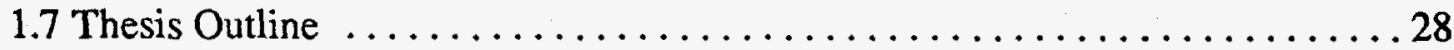

Chapter 2: Experimental Apparatus $\quad 29$

2.1 The Polarized SLC . . . . . . . . . . . . . . . . . . . . . . . 29

2.1.1 Polarized Electron Source $\ldots \ldots \ldots \ldots \ldots \ldots \ldots \ldots \ldots \ldots \ldots \ldots$

2.1.2 Flat Beam Operation ............................ 39

2.1.3 Spin Dynamics $\ldots \ldots \ldots \ldots \ldots \ldots \ldots \ldots \ldots \ldots \ldots \ldots \ldots . . . \ldots \ldots$

2.1.4 Spin Transport ..............................40 40

2.2 Møller Polarimetry .............................. 46

2.2.1 Møller Targets ................................ 48

2.2.2 The Extraction Line Møller Polarimeter $\ldots \ldots \ldots \ldots \ldots \ldots \ldots \ldots .48$

2.2.3 The Linac Møller Polarimeter ..................... 50

2.3 The Compton Polarimeter ............................. 51

2.3.1 The Compton Polarized Target $\ldots \ldots \ldots \ldots \ldots \ldots \ldots \ldots \ldots \ldots \ldots \ldots$

2.3.2 The Cherenkov Detector ........................ 58

2.3.3 The Proportional Tube Detector ......................61 


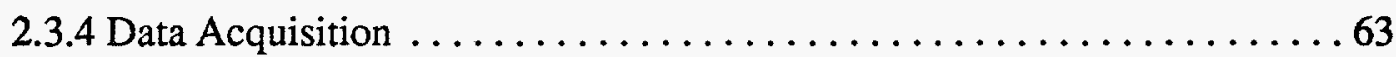

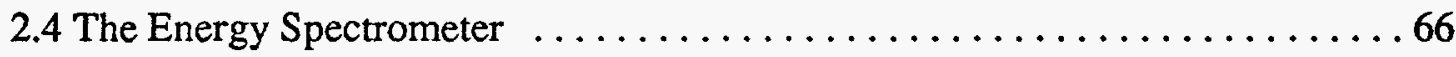

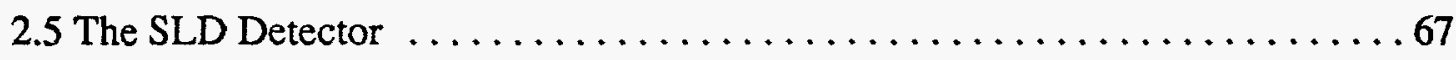

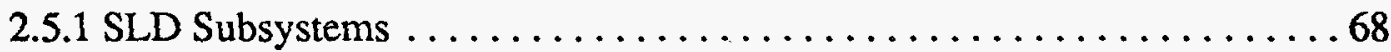

2.5.2 The Liquid Argon Calorimeter $\ldots \ldots \ldots \ldots \ldots \ldots \ldots \ldots \ldots \ldots$

Chapter 3: Compton Polarimetry 73

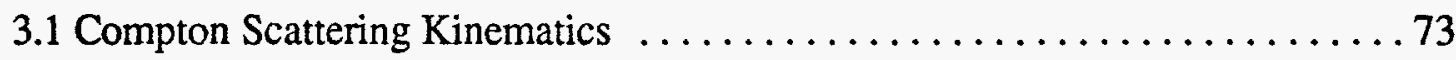

3.1.1 Laboratory Frame Scattering Kinematics . . . . . . . . . . . . . 75

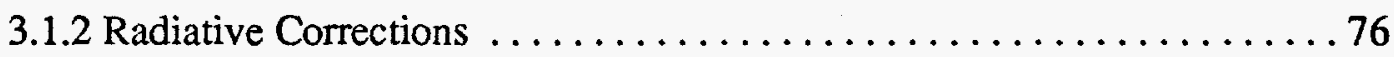

3.2 Extracting the Beam Polarization from Compton Scattering $\ldots \ldots \ldots \ldots . \ldots 77$

3.2.1 The Analyzing Bend Magnet $\ldots \ldots \ldots \ldots \ldots \ldots \ldots \ldots \ldots \ldots \ldots \ldots \ldots \ldots$

3.2.2 The Experimental Asymmetry $\ldots \ldots \ldots \ldots \ldots \ldots \ldots \ldots \ldots \ldots$

3.3 Measuring the Experimental Asymmetry $\ldots \ldots \ldots \ldots \ldots \ldots \ldots \ldots . \ldots 1$

3.3.1 Compton Asymmetries $\ldots \ldots \ldots \ldots \ldots \ldots \ldots \ldots \ldots \ldots \ldots . \ldots 1$

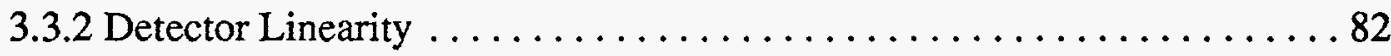

3.3.3 Electronic and Laser Noise Pickup $\ldots \ldots \ldots \ldots \ldots \ldots \ldots \ldots \ldots$

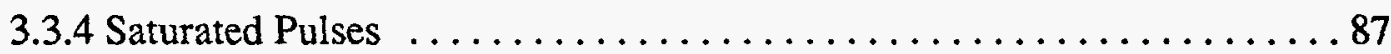

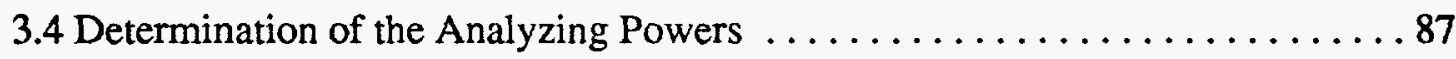

3.4.1 Endpoint Calibration . . . . . . . . . . . . . . . . . . . 88

3.4.2 Zero Asymmetry Point $\ldots \ldots \ldots \ldots \ldots \ldots \ldots \ldots \ldots \ldots \ldots$

3.4.3 The Beamstrahlung Shield $\ldots \ldots \ldots \ldots \ldots \ldots \ldots \ldots \ldots \ldots \ldots \ldots \ldots$

3.4.4 Detector Response Function $\ldots \ldots \ldots \ldots \ldots \ldots \ldots \ldots \ldots \ldots 2$

3.4.5 Interchannel Consistency . . . . . . . . . . . . . . . . 94

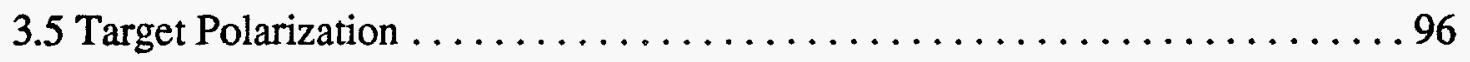

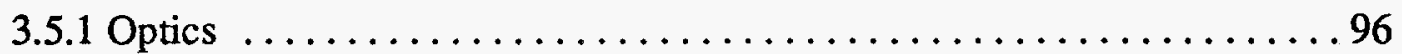

3.5.2 Pre-AUTOPOCKSCAN Analysis $\ldots \ldots \ldots \ldots \ldots \ldots \ldots \ldots \ldots . \ldots 9 . \ldots \ldots$

3.5.3 AUTOPOCKSCAN Analysis $\ldots \ldots \ldots \ldots \ldots \ldots \ldots \ldots \ldots \ldots \ldots \ldots \ldots$

3.6 Absolute Sign Determination . . . . . . . . . . . . . . . . . . . 103 
3.6.1 Sign of the Compton Scattering Asymmetry $\ldots \ldots \ldots \ldots \ldots \ldots . \ldots . \ldots . \ldots$

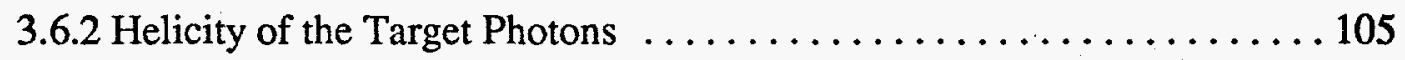

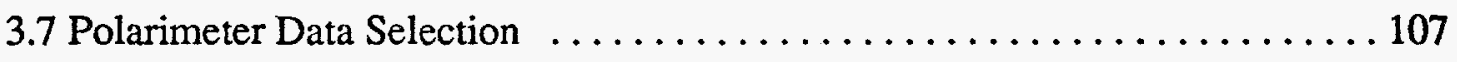

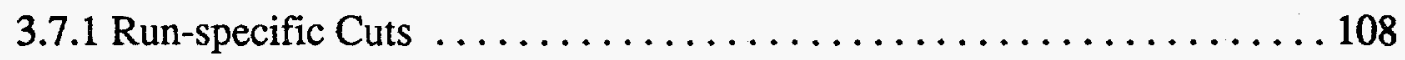

3.7.2 Channel-specific Cuts . ......................... 109

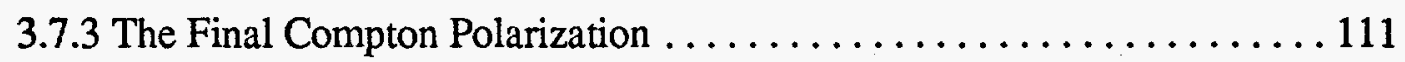

3.8 Polarimeter Systematic Errors . . . . . . . . . . . . . . . . . . 111

3.9 The Luminosity-weighted Polarization $\ldots \ldots \ldots \ldots \ldots \ldots \ldots \ldots \ldots \ldots \ldots \ldots \ldots \ldots \ldots$

\section{Chapter 4: $Z$ Event Selection and Background Estimation $\quad 114$}

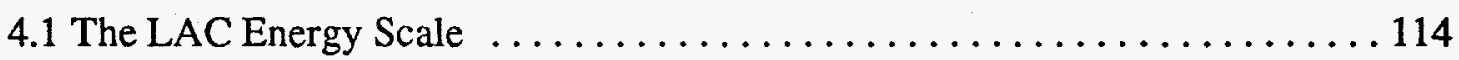

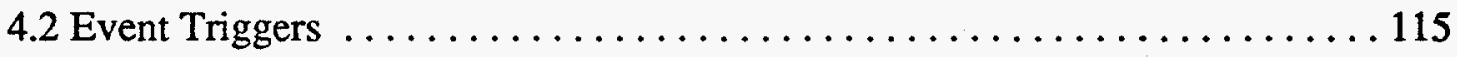

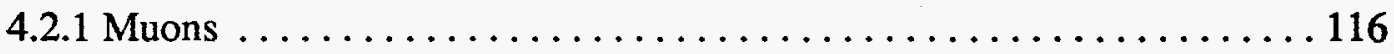

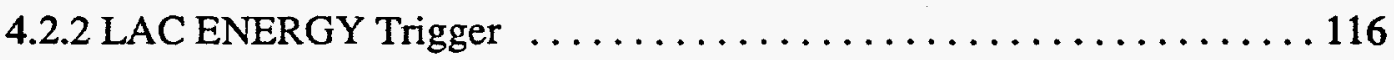

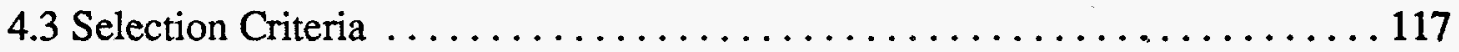

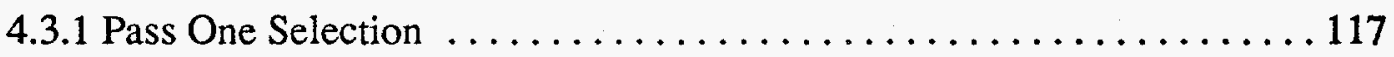

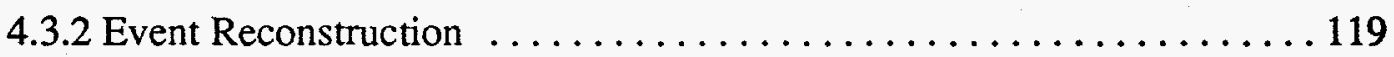

4.3.3 Pass Two Selection ............................ 119

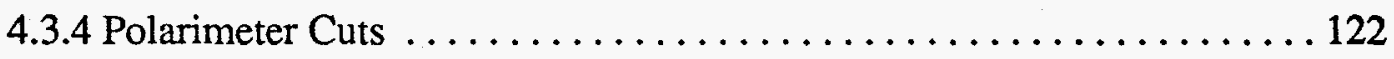

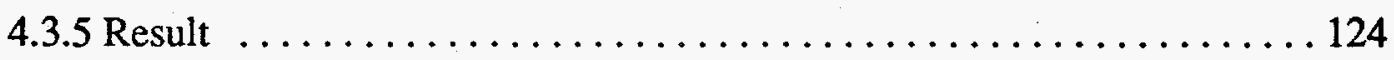

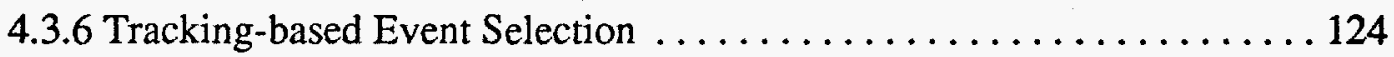

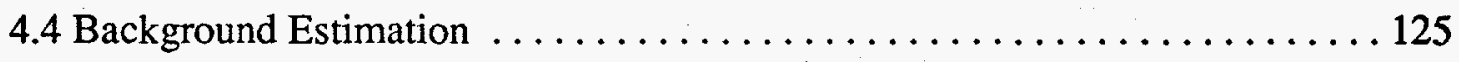

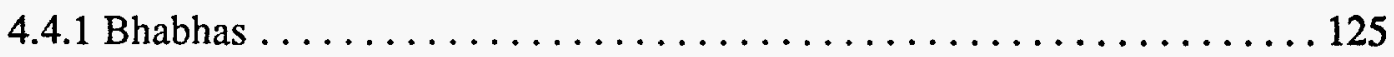

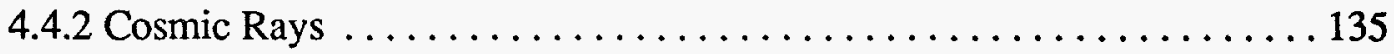

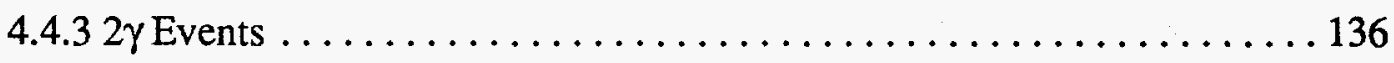

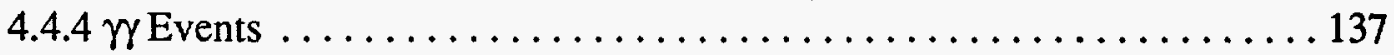

4.4.5 Beam Background Events . . . . . . . . . . . . . . . . . 137

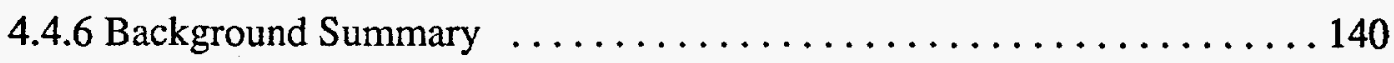

4.5 The Background Asymmetry $\ldots \ldots \ldots \ldots \ldots \ldots \ldots \ldots \ldots \ldots \ldots \ldots \ldots$ 
Chapter 5: Analysis and Results

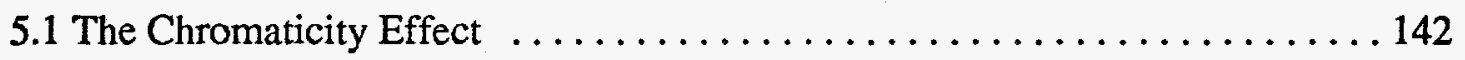

5.1.1 The Chromaticity Model ......................... 143

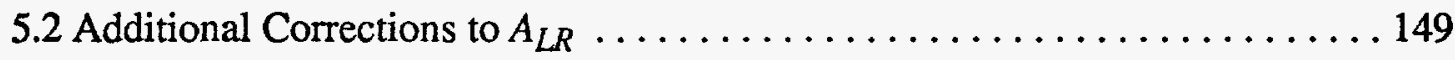

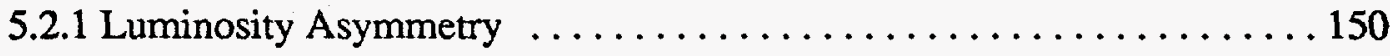

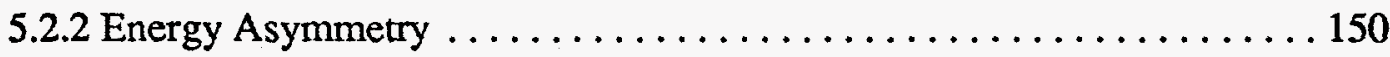

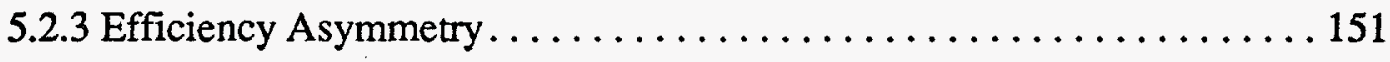

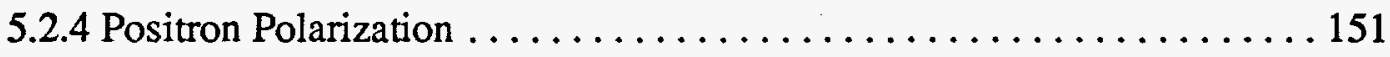

5.2 .5 Summary of Small Corrections . . . . . . . . . . . . . . . 152

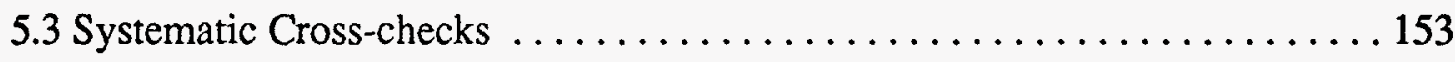

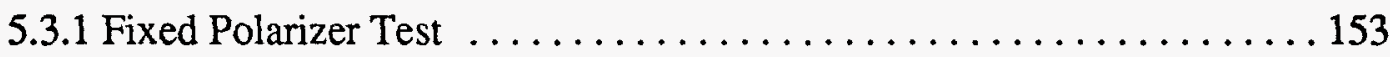

5.3 .2 Current Asymmetry Test ........................ 153

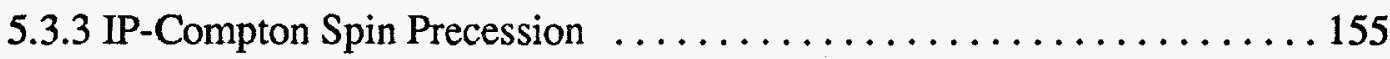

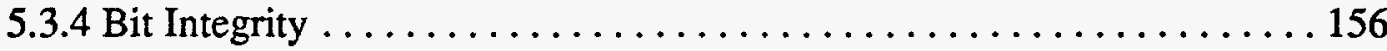

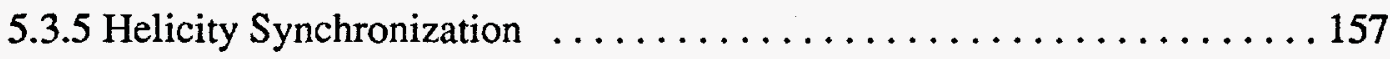

5.3 .6 Cut Bias . . . . . . . . . . . . . . . . . . . . . . 158

5.3.7 Total Systematic Uncertainty $\ldots \ldots \ldots \ldots \ldots \ldots \ldots \ldots \ldots \ldots \ldots$

5.4 Results and Comparisons ..................................... 162

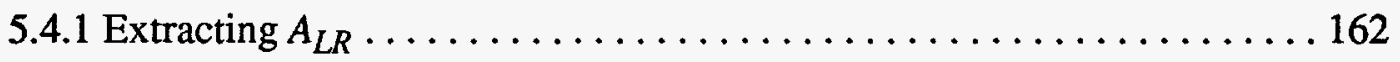

5.4.2 Extracting the Weinberg angle from $A_{L R} \ldots \ldots \ldots \ldots \ldots \ldots \ldots \ldots \ldots \ldots$

5.4 .3 Comparison with LEP data $\ldots \ldots \ldots \ldots \ldots \ldots \ldots \ldots \ldots \ldots \ldots \ldots \ldots \ldots$

5.4.4 Comparison with the Standard Model $\ldots \ldots \ldots \ldots \ldots \ldots \ldots . \ldots . \ldots . \ldots$

5.5 Future Experimental Precision . . . . . . . . . . . . . . . . . 166

$\begin{array}{ll}\text { References } & 168\end{array}$ 


\section{List of Tables}

1-1 The couplings of fermions to the $Z$ in the Standard Model $\ldots \ldots \ldots \ldots \ldots 7$

1-2 Present experimental parameterization of the Standard Model. . . . . . . . . 11

1-3 Properties of various electroweak asymmetries. . . . . . . . . . . . . . 24

2-1 Results of Linac Møller Polarimeter measurements. .............. 51

3-1 1993 Endpoint Scan Results . ..........................899

3-2 Nominal Positions and Analyzing Powers by Period $\ldots \ldots \ldots \ldots \ldots . \ldots 91$

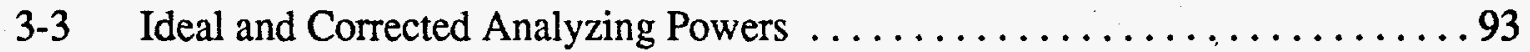

3-4 Thick and Thin Pre-radiator Comparison $\ldots . \ldots \ldots \ldots \ldots \ldots . \ldots . \ldots . \ldots 9$

3-5 Results of best fits to the Cherenkov spectrum. ................ 94

3-6 $\quad P_{y}$ measurements during the pre-AUTOPOCKSCAN era. $\ldots \ldots \ldots \ldots \ldots 100$

3-7 Inferred Electron Helicity during 1993 run $\ldots \ldots \ldots \ldots \ldots \ldots \ldots \ldots$

3-8 Total Systematic Error for Individual Channels and Combined Channels . . . 112

4-1 Results of Method 1 Bhabha background estimate: Barrel Region ........ 131

4-2 Results of Method 1 Bhabha background estimate: Endcap Region ....... 131

4-3 Summary of 1993 background estimate. .................... 140

5-1 Parameters of beam transport simulation. . . . . . . . . . . . . . . 148

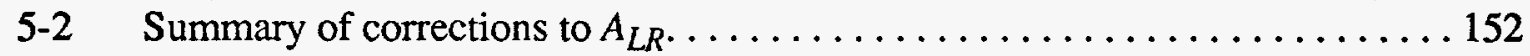

5-3 Total systematic uncertainty on the $A_{L R}$ measurement. ............ 161

5-4 Converting the observed value of $A_{L R}$ into $A_{L R}^{o} \ldots \ldots \ldots \ldots \ldots \ldots \ldots \ldots$

5-5 ZFITTER predictions for top mass and $A_{L R}$ at the $Z$ peak $\ldots \ldots \ldots \ldots 165$ 


\section{List of Figures}

1-1 Fermions in the Standard Model. .........................

1-2 Definition of the weak mixing angle. $\ldots \ldots \ldots \ldots \ldots \ldots \ldots \ldots \ldots$

1-3 Standard Model tree-level process $e^{-} e^{+} \rightarrow f \bar{f} \ldots \ldots \ldots \ldots \ldots \ldots \ldots$

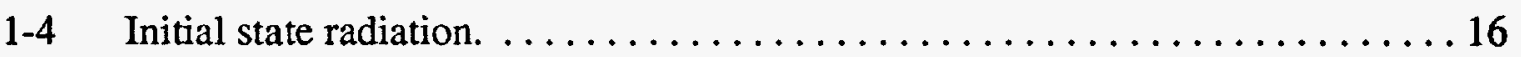

1-5 Radiative corrections to the $Z$ peak cross section. ............... 17

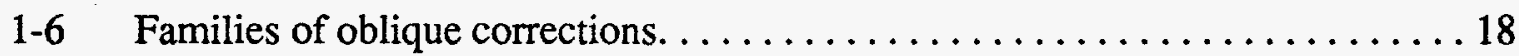

1-7 Lowest order electroweak vertex diagrams. ................. 20

1-8 Lowest order electroweak box diagrams. . . . . . . . . . . . . . 21

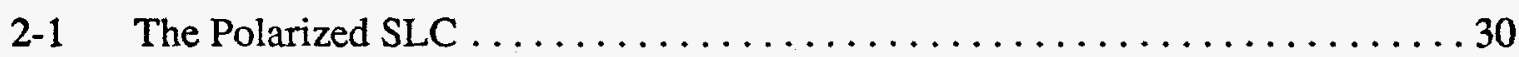

2-2 The SLC polarized electron source $\ldots \ldots \ldots \ldots \ldots \ldots \ldots \ldots \ldots \ldots \ldots \ldots \ldots \ldots \ldots \ldots \ldots$

2-3 Energy level diagram for a Gallium Arsenide photocathode $\ldots \ldots \ldots \ldots . \ldots 32$

2-4 Energy level diagram for a strained Gallium Arsenide photocathode . . . . . . 34

2-5 Measurements of various photocathode materials . . . . . . . . . . . . 35

2-6 The SLC Polarized Electron Gun $\ldots \ldots \ldots \ldots \ldots \ldots \ldots \ldots \ldots \ldots \ldots \ldots$

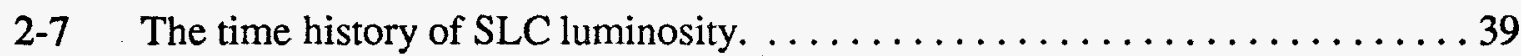

2-8 Spin rotation in the North Damping Ring $\ldots \ldots \ldots \ldots \ldots \ldots \ldots \ldots \ldots$

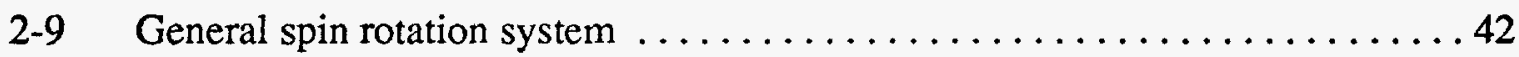

2-10 Vertical orbit and spin components over the first 23 achromat sections . . . . 44

2-11 The dependence of polarization on energy $\ldots \ldots \ldots \ldots \ldots \ldots \ldots \ldots$

2-12 The extraction line Møller polarimeter $\ldots \ldots \ldots \ldots \ldots \ldots \ldots \ldots \ldots . \ldots 49$ 


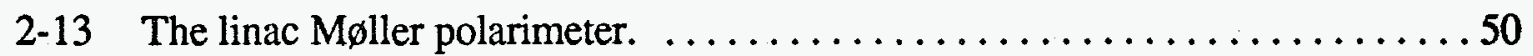

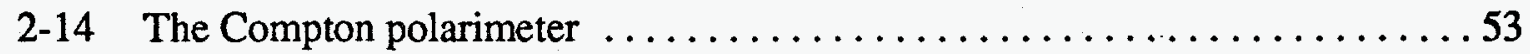

2-15 The CLS laser bench. ............................. 54

2-16 The Compton laser transport system. ................... 56

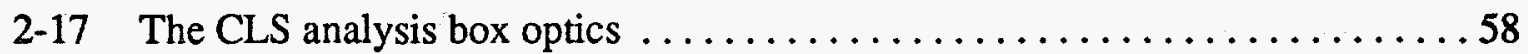

2-18 Overhead view of the Compton Cherenkov and proportional tube detectors. . . 559

2-19 The Compton proportional tube detector. . . . . . . . . . . . . . . 61

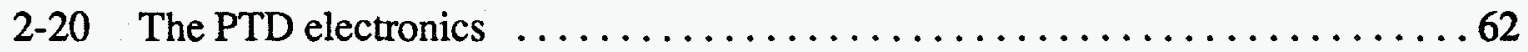

2-21 The PTD linearity from 1993 data. ...................... 63

2-22 Schematic representation of the Compton polarimeter data acquisition . . . . . 64

2-23 The electron extraction line energy spectrometer. . . . . . . . . . . . 66

2-24 Cutaway view of the SLD detector. . . . . . . . . . . . . . . . . . 68

2-25 Quadrant view of the SLD detector. ..................... 71

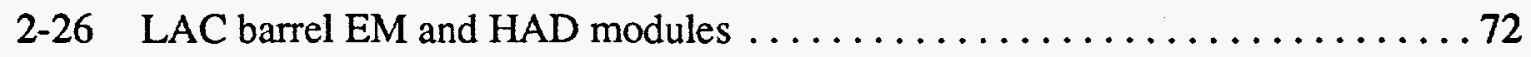

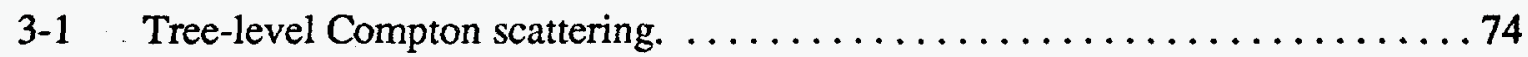

3-2 The Compton cross section and asymmetries. . . . . . . . . . . . . . 77

3-3 The analyzing bend magnet of the Compton polarimeter. ............ 78

3-4 Luminosity-weighted Compton Asymmetries ...................83

3-5 Cherenkov Channel 6 Linearity Calibration $\ldots \ldots \ldots \ldots \ldots \ldots \ldots \ldots . \ldots \ldots$

3-6 Cherenkov Channel 7 Linearity Calibration $\ldots \ldots \ldots \ldots \ldots \ldots \ldots$

3-7 A time history of the laser noise pick-up for Cherenkov channel $6 . \ldots \ldots \ldots 86$

3-8 Monte Carlo results showing the effect of saturated pulses. . . . . . . . . 88

3-9 Compton kinematic edge scan. $\ldots \ldots \ldots \ldots \ldots \ldots \ldots \ldots \ldots \ldots$

3-10 Time history of the location of the Compton zero-asymmetry point. . . . . . 90

3-11 Cherenkov detector response function for channel $7 \ldots \ldots \ldots \ldots \ldots \ldots . \ldots 9$

3-12 Comparison of the measured Compton asymmetry and the theoretical asymmetry, including the EGS detector response function. . . . . . . . . 95

3-13 Introduction of an arbitrary phase shift. $\ldots \ldots \ldots \ldots \ldots \ldots \ldots \ldots \ldots \ldots$

3-14 $P_{\gamma}{ }^{A B}$ in the Pre-AUTOPOCKSCAN era. ....................... 100 
3-15 Time history of $P_{\gamma}$ during the AUTOPOCKSCAN era. $\ldots \ldots \ldots \ldots \ldots 103$

3-16 The 1993 Compton measured polarization. ................... 113

4-1 Pass One events in EHI-ELO space . . . . . . . . . . . . . . . . 118

4-2 Pass One events in Energy versus Imbalance space $\ldots \ldots \ldots \ldots \ldots \ldots \ldots 121$

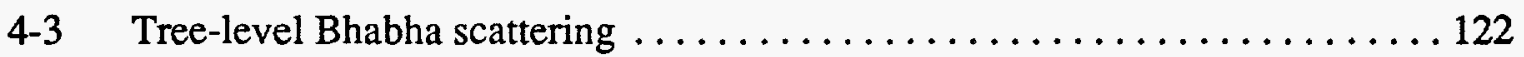

4-4 A summary of the Pass Two event selection. ................. 123

4-5 Normalized cluster multiplicity distributions for Bhabha and hadronic events. 127

4-6 Bhabha selection Method $1 \ldots \ldots \ldots \ldots \ldots \ldots \ldots \ldots \ldots \ldots \ldots . \ldots \ldots$

4-7 The distributions of EEHI1+EEHI2 and EHTOT $\ldots \ldots \ldots \ldots \ldots \ldots \ldots 130$

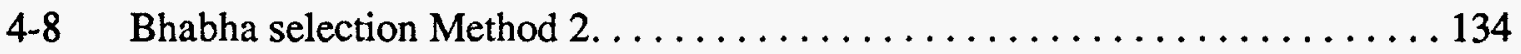

4-9 (A) The $2 \gamma$ process. (B) The $\gamma \gamma$ process. ................... 136

4-10 The Pass One data in $I$ versus $N_{\text {clus }}$ variable space. .............. 138

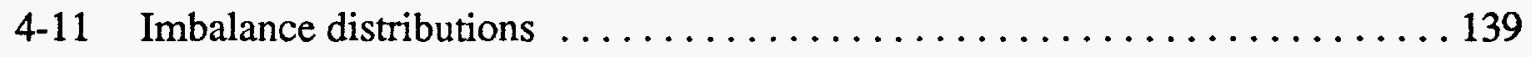

4-12 The angular distribution of the identified Bhabha sample. ............ 141

5-1 (a) $N_{e-}(E),\left(\right.$ b) $\sigma_{\mathrm{y}}(E)$, and (c) $P_{e}(E)$ in the chromaticity model. ........ 145

5-2 The position of the SL-3 collimator vs. the measured Compton polarization. . 147

5-3 Predictions of the chromaticity model. . . . . . . . . . . . . . . . 149

$5-4$ The fixed-polarizer test. . . . . . . . . . . . . . . . . . . . . 154

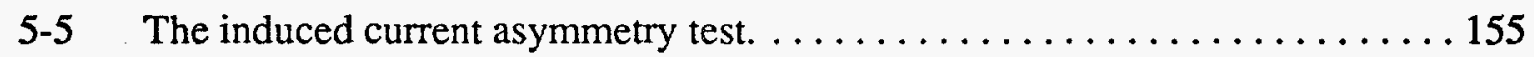

5-6 The PLS/CLS pseudo-random number generator. ............... 156

5-7 The measured asymmetry versus Pass Two cut variables . . . . . . . . . 159

5-8 The measured asymmetry versus other event variables. . . . . . . . . . 160

5-9 A comparison of LEP and SLD $\sin ^{2} \theta_{W}^{\text {eff }}$ measurements. ............ 164

5-10 The projection of electroweak observables in $S-T$ space. $\ldots \ldots \ldots \ldots \ldots 165$

5-11 Standard Model fit to electroweak observables. ............... 167

5-12 The projected error on the effective weak mixing angle. $\ldots \ldots \ldots \ldots \ldots 167$ 


\section{Chapter 1}

\section{Physics Background and Motivation}

The SLAC ${ }^{1}$ Linear Collider (SLC) is a machine designed to accelerate and collide electrons and positrons at high energy ( $50 \mathrm{GeV}$ per beam). Recent upgrades to the SLC electron source allow for the production of spin-polarized electron bunches. The use of polarized electrons at SLC opens a unique window to the world of electroweak interactions. Electroweak asymmetries in particular offer systematically clean experiments with a high sensitivity to new physics. The left-right asymmetry of $Z$ boson production, known as $A_{L R}$, provides a particularly good probe of electroweak radiative corrections. The measurement of $A_{L R}$ is the principle goal of the current SLC/SLD program [1], and a detailed description of this measurement using data recorded during 1993 is the topic of this document.

In this chapter, we will outline the theory of electroweak interactions and its predictions for the $Z$ production cross section and various related asymmetries. We will describe various tests of the theory and the advantages of $A_{L R}$ in particular. Finally, we will discuss the effect of radiative corrections, and a parameterization of these corrections that includes the effects of new physics.

1. Stanford Linear Accelerator Center 


\subsection{The Standard Model}

During the 1960's, Glashow [2], Salam [3], and Weinberg [4] constructed a new model of particle interactions that unified the weak and electromagnetic forces. This model is now known as the Standard Model of Electroweak Interactions, or simply the Standard Model, as it will be referred to throughout this document. This theory is concerned with the unification of the electromagnetic and weak nuclear forces (to be distinguished from Quantum Chromodynamics, or QCD, the theory of the strong nuclear force). The Standard Model has so far proven to be very successful, predicting, among other things, the existence of a massive neutral intermediate vector boson, the $Z$ boson.

\subsubsection{The Gauge Bosons}

The Standard Model is a non-Abelian gauge theory based on the local invariance of the $S U(2) \otimes U(1)$ gauge groups [5]. Each gauge group generator has a field or set of fields associated with it:

$$
\begin{gathered}
W_{\mu}^{1}, W_{\mu}^{2}, W_{\mu}^{3} \text { for } S U(2) \\
B_{\mu} \text { for } U(1)
\end{gathered}
$$

where each field corresponds to a massless gauge boson. Each group generator also has an associated conserved charge. The hypercharge, $Y$, is the conserved charge of the $U(1)$ group, and the weak isospin components, $T^{\alpha}$, are the conserved charges of the $S U(2)$ group. The coupling constant for the $S U(2)$ group is $g$, and the coupling constant for the $U(1)$ group is $g^{\prime} / 2$ (the factor of $1 / 2$ is chosen to simplify later expressions).

The Lagrangian $(\mathcal{L}$ ) for the gauge fields is

$$
\mathcal{L}_{\text {gauge }}=-\frac{1}{4} F_{\mu v}^{c} F^{c \mu v}-\frac{1}{4} f_{\mu v} f^{\mu v}
$$

where the field strength tensors $F$ and $f$ are defined by

$$
\begin{gathered}
F_{\mu v}^{c}=\partial_{v} W_{\mu}^{c}-\partial_{\mu} W_{v}^{c}+g \varepsilon_{a b c} W_{\mu}^{a} W_{\nu}^{b} \\
f_{\mu v}=\partial_{\nu} B_{\mu}-\partial_{\mu} B_{v}
\end{gathered}
$$


In these expressions, $\partial_{\mu}=\partial / \partial x^{\mu}$, and $\varepsilon_{a b c}$ is the completely anti-symmetric Levi-Chivita tensor.

\subsubsection{The Fermions}

The gauge fields of the Standard model interact with two classes of fermions, the quarks and the leptons. These particles occur in left-handed isospin doublets, and right-handed isospin singlets, as illustrated in figure 1-1. For every fermion shown, there also exists an anti-fermion with opposite charge and handedness.

$\begin{array}{lllll}Y=-1 & Y=1 / 3 & Y=-2 & Y=4 / 3 & Y=-2 / 3 \\ {\left[\begin{array}{l}v_{e} \\ e\end{array}\right]_{L}} & {\left[\begin{array}{l}u \\ d\end{array}\right]_{L}} & {[e]_{\mathbb{R}}} & {[u]_{\mathbb{R}}} & {[d]_{\mathbb{R}}} \\ {\left[\begin{array}{l}v_{\mu} \\ \mu\end{array}\right]_{L}} & {\left[\begin{array}{l}c \\ s\end{array}\right]_{L}} & {[\mu]_{\mathrm{R}}} & {[c]_{\mathrm{R}}} & {[s]_{\mathrm{R}}} \\ {\left[\begin{array}{l}v_{\tau} \\ \tau\end{array}\right]_{L}} & {\left[\begin{array}{l}t \\ b\end{array}\right]_{L}} & {[\tau]_{\mathrm{R}}} & {[t]_{\mathrm{R}}} & {[b]_{\mathrm{R}}}\end{array}$

Figure 1-1: Fermions in the Standard Model.

The hypercharges for each particle are as labelled above each column. The third component of weak isospin, $T^{3}$, is $+(1 / 2)$ for the upper elements of the isodoublets, and $-(1 / 2)$ for the lower elements. The isosinglets have $T=0$, which means they do not couple to the $W$ fields. If the neutrinos are massive, there exists an isosinglet neutrino $\left(v_{R}\right)$ state which does not couple to the gauge fields.

The interaction between the fermions and the gauge fields is dictated by the symmetries of the gauge groups and is given by the following Lagrangian:

$$
\mathcal{L}_{f}=R_{f} i \gamma^{\mu}\left(\partial_{\mu}+\frac{i g^{\prime}}{2} Y B_{\mu}\right) R_{f}+L_{f} i \gamma^{\mu}\left(\partial_{\mu}+\frac{i g^{\prime}}{2} Y B_{\mu}+\frac{i g}{2}\left[\vec{\tau} \cdot \vec{W}_{\mu}\right]\right) L_{f}
$$


where $R_{f}=\frac{1}{2}\left(1+\gamma_{5}\right) f$ and $L_{f}=\frac{1}{2}\left(1-\gamma_{5}\right) f$ are general fermion isosinglets and isodoublets, $\gamma^{\mu}$ are the Dirac matrices, and $\vec{\tau}$ is the generator of $S U(2)$ rotations, normally represented by the Pauli matrices.

\subsubsection{Spontaneous Symmetry Breaking}

One difficulty of the theory at this point in its development is that the gauge bosons are all massless. This is all well and good for electromagnetism, but the weak nuclear force is observed to have a finite range which implies that a massive boson is exchanged. Another problem is that global $S U(2)$ invariance prevents us from writing down any mass terms for our fermions, which are of course observed to have mass.

The ingenious solution to this problem was suggested by Higgs (for whom the Higgs boson is named) and others [6]. Weinberg and Salam applied these ideas by introducing a complex doublet of scalar fields

$$
\varphi \equiv\left[\begin{array}{c}
\varphi^{+} \\
\varphi^{0}
\end{array}\right]
$$

which transforms as and $S U(2)$ isodoublet with hypercharge $Y_{\varphi}=1$. We then add some new terms to the overall Lagrangian, first

$$
L_{\text {scalar }}=\left(D^{\mu} \varphi\right)^{\dagger}\left(D_{\mu} \varphi\right)-\kappa^{2}\left(\varphi^{\dagger} \varphi\right)-|\beta|\left(\varphi^{\dagger} \varphi\right)^{2}
$$

where $\kappa$ and $\beta$ are dimensional parameters and the covariant derivative $D_{\mu}$ is defined by

$$
D_{\mu}=\partial_{\mu}+\frac{i g^{\prime}}{2} Y B_{\mu}+\frac{i g}{2}\left[\vec{\tau} \cdot \vec{W}_{\mu}\right]
$$

In addition, we wish to couple the scalar field (the Higgs field) to the fermions, which is accomplished through a Yukawa-type interaction Lagrangian:

$$
\mathcal{L}_{\varphi, f}=-\lambda_{f}\left[R_{f}\left(\varphi^{\dagger} L_{f}\right)+L_{f}\left(\varphi R_{f}\right)\right]
$$

where $\lambda_{f}$ is an arbitrary dimensionless constant. This interaction is invariant under $S U(2) \otimes U(1)$ transformations and is a Lorentz scalar. 
Now we assume $\kappa^{2}<0$ and expand the Higgs field about the minimum of the scalar potential, $v=\sqrt{-\kappa^{2} /|\beta|}$ :

$$
\varphi=\left\langle\varphi_{0}\right\rangle+\left[\begin{array}{c}
0 \\
\eta / \sqrt{2}
\end{array}\right]=\left[\begin{array}{c}
0 \\
v / \sqrt{2}
\end{array}\right]+\left[\begin{array}{c}
0 \\
\eta / \sqrt{2}
\end{array}\right]=\left[\begin{array}{c}
0 \\
(v+\eta) /(\sqrt{2})
\end{array}\right]
$$

where $\left\langle\varphi_{0}\right\rangle$ is the vacuum expectation value of the scalar field, which breaks both the isospin and hypercharge symmetries, and $\eta$ is the perturbation of the vacuum. The only remaining symmetry in the theory is the strange combination of operators

$$
\left(T^{3}+\frac{1}{2} Y\right)\left\langle\varphi_{0}\right\rangle=Q\left\langle\varphi_{0}\right\rangle=0
$$

where $Q$ is now identifiable as the electric charge operator. This is the essence of the spontaneous-symmetry breaking process: the original gauge symmetries are broken, leaving only the physically observed conserved quantity, the electric charge.

Expanding various terms in the Lagrangian about the vacuum expectation value of the Higgs field has very interesting results:

$$
\mathcal{L}_{\text {scalar }}=\frac{1}{2}\left(\partial^{\mu} \eta\right)\left(\partial_{\mu} \eta\right)-\kappa^{2} \eta^{2}+\frac{v^{2}}{8}\left[g^{2}\left|W_{\mu}^{1}-i W_{\mu}^{2}\right|^{2}+\left(g^{\prime} B_{\mu}-g W_{\mu}^{3}\right)^{2}\right]
$$

plus interaction terms. The $\eta$ field has become the physical manifestation of the Higgs boson with mass $m_{H}^{2}=-2 \kappa^{2}$. We further identify the charged gauge fields

$$
W_{\mu}^{ \pm} \equiv \frac{W_{\mu}^{1} \mp W_{\mu}^{2}}{\sqrt{2}}
$$

with a mass of $m_{W^{ \pm}}=g v / 2$, and a neutral gauge field

$$
Z_{\mu}=\cos \theta_{W} W_{\mu}^{3}-\sin \theta_{W} B_{\mu}
$$

where we have introduced the weak mixing angle, $\theta_{W}$, which is related to the original gauge coupling constants as shown in figure 1-2. 


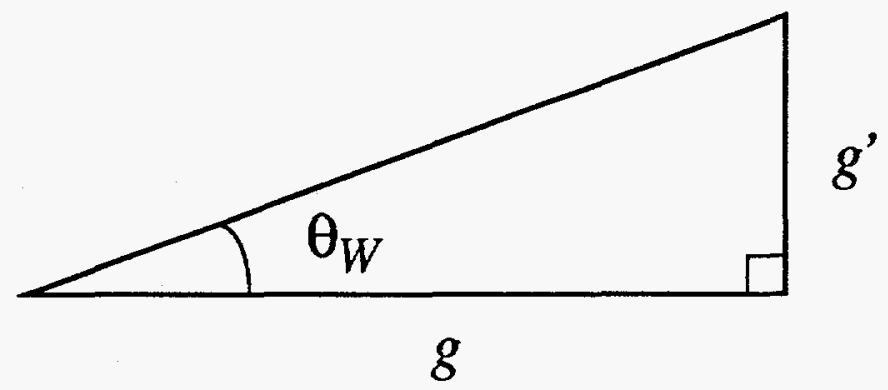

Figure 1-2: Definition of the weak mixing angle.

This neutral field has a mass $m_{Z}=m_{W} / \cos \theta_{W}$. The orthogonal combination of the original gauge fields yields an additional neutral field

$$
A_{\mu}=\sin \theta_{W} W_{\mu}^{3}+\cos \theta_{W} B_{\mu},
$$

which is massless. $A_{\mu}$ may now be identified as the vector potential of the photon field.

The Yukawa potential of equation (1.7) now has the form

$$
\mathcal{L}_{\varphi, f}=\frac{-\lambda_{f} v}{\sqrt{2}} \bar{f} f+\frac{-\lambda_{f} \eta}{\sqrt{2}} \bar{f} f
$$

which introduces a mass term for the fermions, as well an interaction term for fermions with the physical Higgs, $\eta$. Unfortunately, the constants $\lambda_{f}$ are arbitrary and offer no real insight into the question of fermion mass.

The interaction Lagrangian now has two distinct parts: the charged-current part and the neutral current part. The charged-current interaction for leptons has the following form:

$$
\mathcal{L}_{\text {charged }}=\frac{-g}{2 \sqrt{2}}\left[\nabla_{f} \gamma^{\mu}\left(1-\gamma_{5}\right) f W_{\mu}^{+}+\bar{f} \gamma^{\mu}\left(1-\gamma_{5}\right) v_{f} W_{\mu}^{-}\right]
$$


and the neutral-current interaction for leptons, expressed in terms of electroweak operators, is given by

$$
\mathcal{L}_{\text {neutral }}=g \sin \theta_{W} f \gamma^{\mu} f A_{\mu}-g \sin \theta_{W} \bar{f} \gamma^{\mu}\left[\frac{\left(1-\gamma_{5}\right)}{\sin 2 \theta_{W}} T_{f}^{3}-\frac{2 \sin ^{2} \theta_{W}}{\sin 2 \theta_{W}} Q_{f}\right] f Z_{\mu}
$$

This interaction can be compactly written as

$$
\mathcal{L}_{\text {neutral }}=e \bar{f} \gamma^{\mu} f A_{\mu}-e \bar{f} \gamma^{\mu}\left[v_{f}-a_{f} \gamma_{5}\right] f Z_{\mu}
$$

where we have identified the electric charge, $e=g \sin \theta_{W}$, and the vector $\left(v_{f}\right)$ and axial vector $\left(a_{f}\right)$ coupling constants are defined as follows

$$
\begin{aligned}
& v_{f}=\left(T_{f}^{3}-2 \sin ^{2} \theta_{W} Q_{f}\right) / \sin 2 \theta_{W} . \\
& a_{f}=T_{f}^{3} / \sin 2 \theta_{W}
\end{aligned}
$$

The values of these constants for all fermions in the theory are listed in table 1-1.

\begin{tabular}{|c|c|c|}
\hline Fermion & $a_{f} \cdot \sin 2 \theta_{W}$ & $v_{f} \cdot \sin 2 \theta_{W}$ \\
\hline \hline$v_{e}, v_{\mu}, v_{\tau}$ & $\frac{1}{2}$ & $\frac{1}{2}$ \\
\hline$e^{-}, \mu^{-}, \tau^{-}$ & $-\frac{1}{2}$ & $-\frac{1}{2}+2 \sin ^{2} \theta_{W}$ \\
\hline$u, c, t$ & $\frac{1}{2}$ & $\frac{1}{2}-\frac{4}{3} \sin ^{2} \theta_{W}$ \\
\hline$d, s, b$ & $-\frac{1}{2}$ & $-\frac{1}{2}+\frac{2}{3} \sin ^{2} \theta_{W}$ \\
\hline
\end{tabular}

Table 1-1: The vector and axial-vector couplings of fermions to the $Z$ in the Standard Model

\subsection{The $Z$ Production Cross Section}

At this point we wish to demonstrate what physics can be explored with a polarized electron/positron collider. The Standard Model provides the tools necessary to calculate the cross section for the process $e^{-} e^{+} \rightarrow f \bar{f}$, which is diagrammed in figure 1-3. The special 
case of $e^{-} e^{+}$final states is excluded, because this necessitates the addition of a $t$-channel photon exchange diagram, a complication we wish to avoid.
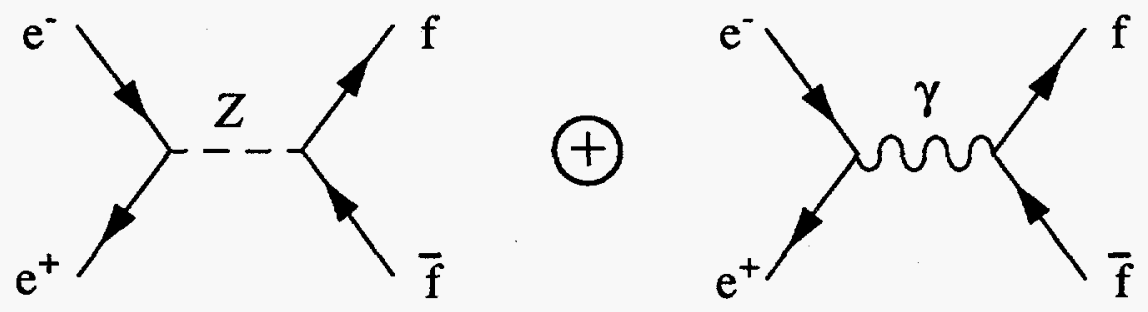

Figure 1-3: Standard Model tree-level process $e^{-} e^{+} \rightarrow f \bar{f}$.

There are three parts to the total cross section: the pure photon exchange, the pure $Z$ exchange, and the interference term, which we consider in turn. However, we must first define the beam polarization, $P$, in order to include the effects of polarized electrons in the $Z$ cross section in a meaningful way.

\subsubsection{Defining Polarization}

We define the parameter $P(\hat{n})$ for a group of fermions as

$$
P(\hat{n})=\frac{N(\text { spins parallel to } \hat{n})-N(\text { spins antiparallel to } \hat{n})}{N(\text { spins parallel to } \hat{n})+N(\text { spins antiparallel to } \hat{n})} \text {. }
$$

The polarization of the group is $P(\hat{s})$, where $\hat{s}$ is the direction for which $P(\hat{n})$ is maximized. The longitudinal polarization, $P_{z}$, of the bunch is equal to $P(\hat{z})$, and the transverse polarization is then $P_{t}=\sqrt{[P(\hat{S})]^{2}-[P(\hat{z})]^{2}}$. Right-handed particles (particles whose spins are aligned with their momenta) have $P_{z}=+1 ;$ Left-handed particles have $P_{z}=-1$.

\subsubsection{The Photon Exchange}

The photon exchange term is given by

$$
\left.\frac{d \sigma}{d \Omega}\right|_{\gamma}=\frac{\alpha^{2}}{4 s}\left[1+\cos ^{2} \theta\right] Q_{f}^{2}
$$


where $\alpha$ is the fine-structure constant, equal to the electric charge-squared divided by $4 \pi$, $s$ is equal to the center-of-mass energy-squared, and $\theta$ is the polar angle and $Q_{f}$ the electric charge of the emergent fermion.

At the $Z$ pole $\left(s=m_{Z}^{2}\right.$ ) the photon exchange cross section is approximately 800 times smaller than the $Z$-exchange. The photon exchange is a parity-conserving process that dilutes any electroweak asymmetry and must be accounted for in a precision measurement.

\subsubsection{The $Z$ Exchange}

Given our definition of polarization, the complete polarization-dependent $Z$ pole cross section in the limit of light final state fermions $(\beta \rightarrow 1)$ is

$$
\begin{aligned}
& \left.\frac{d \sigma}{d \Omega}\right|_{Z}=\frac{\alpha^{2}}{4} \frac{s}{\left(s-m_{Z}^{2}\right)^{2}+m_{Z}^{2} \Gamma_{Z}^{2}} \times \\
& \left\{\left(1-P_{z}^{+} P_{z}^{-}\right)\left[\left(1+c^{2}\right)\left(v_{e}^{2}+a_{e}^{2}\right)\left(v_{f}^{2}+a_{f}^{2}\right)+8 c v_{e} a_{e} v_{f} a_{f}\right]\right. \\
& +\left(P_{z}^{+}-P_{z}^{-}\right)\left[2\left(1+c^{2}\right) v_{e} a_{e}\left(v_{f}^{2}+a_{f}^{2}\right)+4 c\left(v_{e}^{2}+a_{e}^{2}\right) v_{f} a_{f}\right] \\
& \left.+P_{t}^{+} P_{t}^{-} \cos \Phi\left(1-c^{2}\right)\left(v_{e}^{2}-a_{e}^{2}\right)\left(v_{f}^{2}+a_{f}^{2}\right)\right\}
\end{aligned}
$$

where $\alpha$ is the electromagnetic fine structure constant, $s$ is the square of the center of mass collision energy, $\Gamma_{Z}$ is the resonant width of the $Z$, and $c$ is the cosine of the polar angle of the outgoing fermion. The superscripts "-" and "+" refer to the electron and positron beams, respectively. The subscripts " $z$ " and " $t$ " refer to longitudinal and transverse polarization components, respectively. The angle $\Phi$ is defined by $\Phi=2 \phi-\phi^{-}-\phi^{+}$, where $\phi$ is the azimuthal angle of the outgoing fermion, and $\phi^{ \pm}$are the azimuthal angles of the electron and 
positron transverse polarizations. Equation (1.21) simplifies somewhat in the case of longitudinal electron polarization only:

$$
\begin{aligned}
& \left.\frac{d \sigma}{d \Omega}\right|_{Z}=\frac{\alpha^{2}}{4} \frac{s}{\left(s-m_{Z}^{2}\right)^{2}+m_{Z}^{2} \Gamma_{Z}^{2}} \times \\
& \left\{\left(1+c^{2}\right)\left(v_{e}^{2}+a_{e}^{2}\right)\left(v_{f}^{2}+a_{f}^{2}\right)+8 c v_{e} a_{e} v_{f} a_{f}\right. \\
& \left.-P_{e}\left[2\left(1+c^{2}\right) v_{e} a_{e}\left(v_{f}^{2}+a_{f}^{2}\right)+4 c\left(v_{e}^{2}+a_{e}^{2}\right) v_{f} a_{f}\right]\right\}
\end{aligned}
$$

where the longitudinal polarization of electrons is now denoted as $P_{e}$, a definition that will be used throughout this document. We can integrate equation (1.22) over the symmetric detector acceptance limit $x$ (a symmetric $\cos \theta$ limit) to find

$$
\begin{aligned}
\sigma_{Z} & =\left[\frac{\alpha^{2} s}{4}\right] \frac{\left(v_{f}^{2}+a_{f}^{2}\right)\left(v_{e}^{2}+a_{e}^{2}\right)}{\left(s-m_{Z}^{2}\right)^{2}+m_{Z}^{2} \Gamma_{Z}^{2}} F(x) \times\left[1-P_{e} \cdot \frac{2 v_{e} a_{e}}{\left(v_{e}^{2}+a_{e}^{2}\right)}\right] . \\
& =\sigma_{\text {unp }}\left[1-P_{e} A_{e}\right]
\end{aligned}
$$

We will show that the asymmetry term $A_{e}$ is equivalent to $A_{L R}$ in section 1.3.1. The geometric factor $F(x)$ is given by

$$
F(x)=4 \pi\left(x+\frac{x^{3}}{3}\right)
$$

\subsubsection{Z- $\gamma$ Interference}

The $Z-\gamma$ interference term is given by

$$
\begin{aligned}
& \left.\frac{d \sigma}{d \Omega}\right|_{Z \gamma}=\frac{-Q_{f} \alpha^{2}}{2}\left(1-\frac{m_{Z}^{2}}{s}\right) \frac{s}{\left(s-m_{Z}^{2}\right)^{2}+m_{Z}^{2} \Gamma_{Z}^{2}} \times \\
& \left\{\left(1+c^{2}\right) v_{e} v_{f}+2 c a_{e} a_{f}-P_{e}\left[\left(1+c^{2}\right) v_{f} a_{e}+2 c v_{e} a_{f}\right]\right\}
\end{aligned}
$$


which vanishes rigorously at the $Z$ pole. However, due to the effects of initial state radiation, no real experiment takes place at exactly the $Z$ pole energy. Therefore the interference diagram, which does have parity-violating terms, must be taken into account for precision electroweak measurements.

\subsection{Standard Model Tests}

It is the goal of electroweak physics to test the Standard Model to the limits of experimental precision. However, no single measurement is sufficient for this task.

\begin{tabular}{|c|c|c|}
\hline Parameter & Standard Model Dependence & Value \\
\hline \hline$\alpha$ & $g g^{\prime} / 4 \pi\left(g^{2}+g^{\prime 2}\right)$ & $1 / 137.0359895(61)[7]$ \\
\hline$G_{F}$ & $1 /\left[\sqrt{2} v^{2}\right]$ & $1.16637(2) \times 10^{-5} \mathrm{GeV}^{-2}[7]$ \\
\hline$m_{Z}$ & $\sqrt{\left(g^{2}+g^{\prime 2}\right) v^{2} / 4}$ & $91.190(4) \mathrm{GeV}[8]$ \\
\hline
\end{tabular}

Table 1-2: Present experimental parameterization of the Standard Model.

At tree level, low-energy $(\sqrt{s} \leq 100 \mathrm{GeV})$ electroweak phenomena depend on three parameters of the Standard Model: the gauge coupling constants $g$ and $g$, and the vacuum expectation value of the Higgs field, $v / \sqrt{2}$. These parameters have been accurately specified by the measurements listed in table 1-2.

However, beyond tree-level, higher order (loop-level) corrections involve summations over all heavy particles (heavy meaning $\mathrm{m}^{2} / \mathrm{s}$ for a given particle is not negligible). Therefore, electroweak observables are sensitive to the Standard Model Higgs boson mass $\left(m_{H}\right)$ and the top quark mass $\left(m_{t}\right)$, as well as to heavy physics beyond the Standard Model. At present, the Higgs mass is unknown, and the top mass is only weakly specified [9]. As a result of the loop-level dependence on unknown parameters, measuring a single observable does not test the Standard Model. To make a meaningful test, we must measure several quantities with different dependences on $m_{t}$ and $m_{H}$. 
There are two possible approaches to such a test. One is to simultaneously fit all measurements to the model and use this fit to estimate the unknown parameters. The goodness of fit is then taken as an indicator of the validity of the model. The problem with this approach is that it is not particularly sensitive to the new physics, which may manifest itself in only a few observables. The second approach involves parameterizing deviations from the Standard Model in a model-independent way, and including such parameters in the measurement fits. This is a more direct method for searching for new physics, and will be discussed further in section 1.4.2.

To reiterate: $A_{L R}$ does not on its own test the Standard Model or make any predictions about the unknown parameters such as the top quark mass. It must be considered in context with other measurements in order to draw conclusions about the Standard Model or new physics. Therefore we present an overview of some other electroweak measurements in order to provide this context.

\subsubsection{Electroweak Asymmetries}

The structure of the polarized $Z$ cross section allows for various probes of the fermion coupling constants. By measuring cross section asymmetries, one avoids the systematic errors associated with the measurement of an absolute cross section. There are many asymmetry possibilities, some of which make use of polarized initial states.

\section{General Fermion Asymmetry}

One of the basic asymmetries is the left-right $Z$ production asymmetry for fermions. At the $Z$ pole, we rigorously define the general fermion asymmetry, $A_{f}$, as

$$
A_{f} \equiv \frac{\sigma\left(f_{L} \bar{f} \rightarrow Z\right)-\sigma\left(f_{R} \bar{f} \rightarrow Z\right)}{\sigma\left(f_{L} \bar{f} \rightarrow Z\right)+\sigma\left(f_{R} \bar{f} \rightarrow Z\right)}=\frac{2 v_{f} a_{f}}{v_{f}^{2}+a_{f}^{2}}
$$

where $\sigma\left(f_{L[R]} \bar{f} \rightarrow Z\right)$ is the total cross section for the given tree-level process at the $Z$ pole when the incident fermion is left[right]-handed. This particular combination of coupling constants appears again and again in electroweak physics. 


\section{The Left-Right Asymmetry}

The left-right asymmetry of $Z$ boson production is measured by observing the final states from the process $e^{+} e^{-} \rightarrow Z \rightarrow f \bar{f}$, and forming the number asymmetry between states created with left-handed electrons $\left(P_{e}=-1\right)$ and right-handed electrons $\left(P_{e}=1\right)$ [10]:

$$
A_{L R}=\frac{\sum_{f} \int_{-x_{f}}^{x_{f}} \frac{d \sigma}{d c}\left(P_{e}=-1\right) d c-\sum_{f} \int_{-x_{f}}^{x_{f}} \frac{d \sigma}{d c}\left(P_{e}=1\right) d c}{\sum_{f} \int_{-x_{f}}^{x_{f}} \frac{d \sigma}{d c}\left(P_{e}=-1\right) d c+\sum_{f} \int_{-x_{f}}^{x_{f}} \frac{d \sigma}{d c}\left(P_{e}=1\right) d c}
$$

where $c$ is the cosine of emergent fermion polar angle, $x_{f}$ is the limit of the detector acceptance for a given fermion final state, and we sum over all fermion final states (excepting $\left.\mathrm{e}^{+} \mathrm{e}^{-}\right)$. Substitution of (1.22) reduces this expression to

$$
A_{L R}=\frac{2 v_{e} a_{e}}{v_{e}^{2}+a_{e}^{2}}=A_{e}
$$

Thus the left-right asymmetry $A_{L R}$ is equivalent to $A_{f}$ defined in equation (1.26), when the initial state fermions are electrons and positrons. The beauty of the $A_{L R}$ measurement is now apparent. The final states couplings have dropped out of the asymmetry, as well as any dependence on detector acceptance.

\section{Forward-Backward Asymmetries}

In order to appreciate the properties of $A_{L R}$, one should consider at some other electroweak asymmetries that do not involve polarized electrons. Examination of the $Z$ cross section indicates some interesting angular dependence even when $P_{e}=0$, namely the terms dependent on $\cos \theta$. This indicates that final state distributions are not symmetric in the forward and backward hemispheres. The forward-backward asymmetry is defined by [11]

$$
A_{F B}^{f}=\frac{\int_{0}^{1} \frac{d \sigma}{d c} d c-\int_{-1}^{0} \frac{d \sigma}{d c} d c}{\int_{-1}^{1} \frac{d \sigma}{d c} d c}=\frac{3}{4} A_{e} A_{f}
$$


The value of this asymmetry varies from $\approx 1 \%$ for charged leptons to $\approx 9 \%$ for $d$-type quarks. It is experimentally easiest to measure $A_{F B}$ for leptons; however, these quantities are the least sensitive to $\sin ^{2} \theta_{W}$. The forward-backward asymmetry for quarks has higher sensitivity, but many more experimental difficulties in terms of final state identification, residual backgrounds, and QCD corrections. The $b$-quark final state offers high sensitivity and reasonable identification through vertexing, but is affected by uncertainties in $b-\bar{b}$ mixing.

\section{Tau Polarization Asymmetry}

The fact that fermions produced from $Z$ decays tend to be highly polarized can be exploited to form the fermion polarization asymmetry [11]

$$
P_{f}(\cos \theta)=\frac{\frac{d \sigma}{d \Omega}\left(f_{L}\right)-\frac{d \sigma}{d \Omega}\left(f_{R}\right)}{\frac{d \sigma}{d \Omega}\left(f_{L}\right)+\frac{d \sigma}{d \Omega}\left(f_{R}\right)}=\frac{2 A_{e} \cos \theta+A_{f}\left(1+\cos ^{2} \theta\right)}{\left(1+\cos ^{2} \theta\right)+2 A_{e} A_{f} \cos \theta}
$$

which, when integrated over symmetric azimuthal acceptance, eliminates the dependence on the initial state couplings to yield

$$
P_{f}=A_{f} .
$$

The Standard Model $\tau$-lepton decays via a pure V-A current into low multiplicity final states and is the most obvious candidate for a polarimeter. In principle, the angular distributions of the various tau decay modes are functions of $P_{\tau}$, and one determines the polarization by fitting to these distributions. In practice, the cross section and acceptance for this process is statistically limited, and the identification of the final states and backgrounds makes for a non-trivial analysis with many systematic effects to consider.

\subsubsection{Width of the $Z$ Resonance}

In addition to the electroweak asymmetries described thus far, there are many more electroweak observables that one could include in a Standard Model fit. For instance, the mass of the $W$ boson, extracted from transverse lepton mass distributions in proton-antiproton colliders, or the total hadronic cross section of the $Z$ boson, extracted from 
measurements of the $Z$ lineshape. We present details about one such observable, $\Gamma_{Z}$, because it is one of the most precisely measured and is also somewhat orthogonal to $A_{L R}$ in terms of sensitivity to new physics.

The width of the $Z$ resonance, $\Gamma_{Z}$, appears in the $Z$ propagator term of equation (1.21), and eliminates the singularity in the cross section at $s=m_{Z}^{2}$. This width has a tree-level dependence on the Standard Model parameters and the particle content of the theory. The total width is the sum of the partial widths, $\Gamma_{f \bar{f}}$, for the decay into each fermion-antifermion final state:

$$
\Gamma_{Z}=\sum_{f} \Gamma_{f f}=\frac{G_{F} m_{Z}^{3}}{24 \sqrt{2} \pi} \sum_{f} C_{f}\left[v_{f}^{2}+a_{f}^{2}\right]
$$

The constant $C_{f}$ is defined up to first-order corrections by

$$
C_{f}=\begin{array}{rr}
1+\frac{3 \alpha}{4 \pi} Q_{f}^{2} & \text { for leptons } \\
3 \cdot\left[1+\frac{3 \alpha}{4 \pi} Q_{f}^{2}+\frac{\alpha_{s}}{\pi}\right] & \text { for quarks }
\end{array}
$$

where $\alpha_{s}$ is the strong QCD coupling constant.

The determination of $\Gamma_{Z}$ (along with $m_{Z}$ ) is made by measuring the cross section for the process $e^{+} e^{-} \rightarrow Z \rightarrow f \bar{f}$ as a function of center-of-mass energy, and fitting the results to the theoretical $Z$ lineshape, with $\Gamma_{Z}$ among the free parameters. This method is insensitive to the absolute energy and normalization, but is sensitive to relative energy and luminosity of each scan point. The present best determination of $\Gamma_{Z}$ comes from a combined measurement of the LEP ${ }^{1}$ collaborations [8]

$$
\Gamma_{Z}=2.4969 \pm 0.0038 \mathrm{GeV}
$$

which probes the Standard Model with a sensitivity comparable to that of the $A_{L R}$ measurement presented in this document.

1. The LEP (Large Electron Project) collaboration consists of four detector experiments, ALEPH, DELPHI, L3, and OPAL at the international laboratory CERN in Geneva, Switzerland. 


\subsection{Radiative Corrections}

There are two principle types of radiative corrections: those involving the emission of real particles from the initial state, called initial state radiation (or sometimes external radiation), and those involving the emission and absorption of virtual particles, called the virtual corrections. Of the virtual corrections, we will discuss three distinct types: the oblique corrections, the vertex corrections, and box corrections.

\subsubsection{Initial State Radiation}

Electrons and positrons in the initial state have some probability for emitting photons before interacting with each other, as illustrated in figure 1-4. This has the effect of lowering the effective center-of-mass energy, which changes the total cross section [12]. In addition, the contribution of the $Z-\gamma$ interference term is also a function of energy. As a result, the measured left-right asymmetry is also affected by initial state radiation.
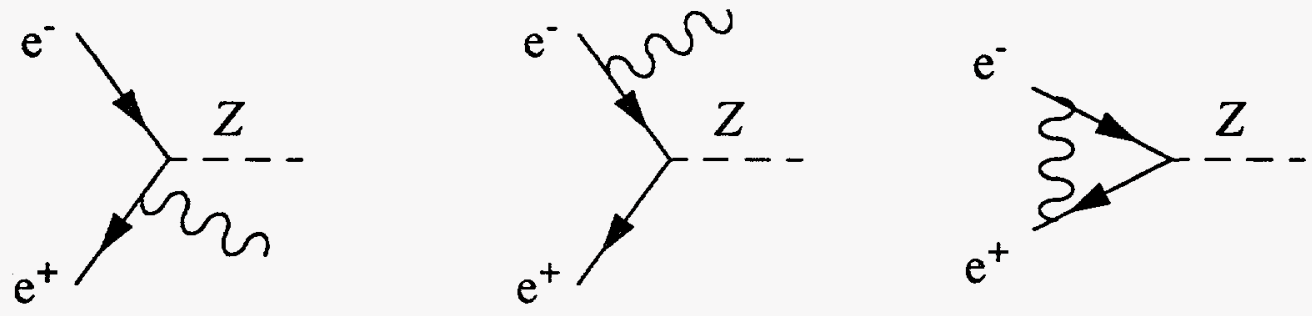

Figure 1-4: Initial state radiation.

The effects of initial state radiation on the observed cross section are incorporated by using the electron structure function [13], $D_{e}(x, s)$, which is the probability that the electron will retain a fraction $x$ of its initial momentum in a collision at the uncorrected center-ofmass energy, $s$. Then the effective center of mass energy is given by $s^{\prime}=x_{1} x_{2} s$ and the observed cross section is

$$
\sigma_{\text {observed }}=\int_{0}^{1} D_{e}\left(x_{1}, s\right) d x_{1} \int_{0}^{1} D_{e}\left(x_{2}, s\right) \sigma_{\text {tree }}\left(x_{1} x_{2} s\right) d x_{2}
$$


At tree level, the function $D_{e}(x, s)$ was calculated by Bonneau and Martin [14], which leads to a $-29 \%$ correction to the tree-level cross section, shown in figure 1-5. Such a large correction suggests that higher order terms are called for [15]

$$
\begin{gathered}
D_{e}(x)=\beta(1-x)^{\beta-1}\left(1+\frac{3}{4} \beta\right)-\frac{\beta}{2}(1-x) \\
\text { where } \quad \beta=\frac{\alpha}{\pi}\left(\log \frac{s}{m_{e}^{2}}-1\right)
\end{gathered}
$$

The effect of this correction is also shown in figure 1-5.

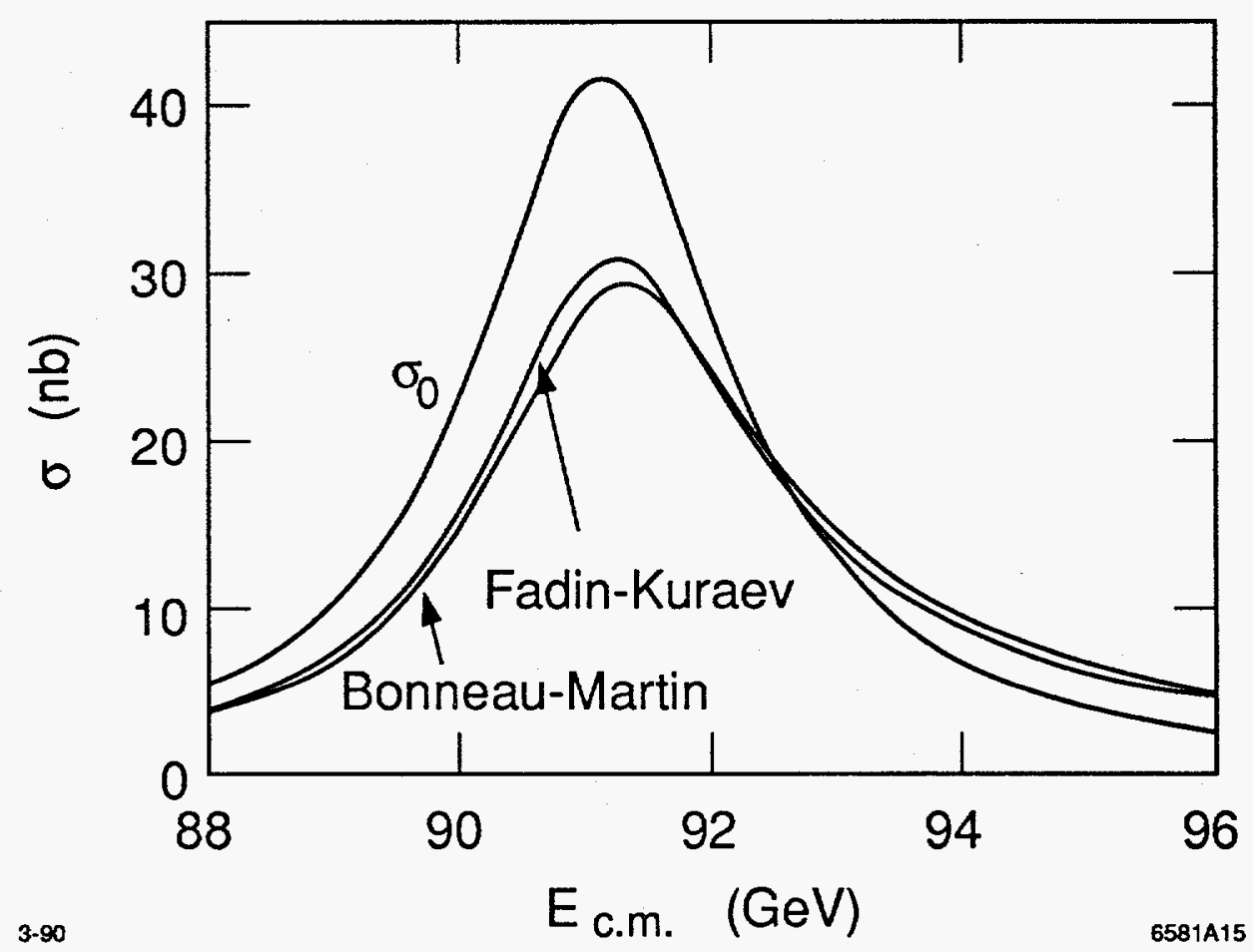

Figure 1-5: Radiative corrections to the $Z$ peak cross section.

\subsubsection{Oblique Corrections}

Vacuum polarizations affect the interactions by modifying the gauge boson propagators (and thereby the coupling constants). This is why they are referred to as oblique corrections, as compared to the direct (vertex and box) corrections which modify the form of the inter- 
actions themselves. The oblique corrections have the largest effect on electroweak asymmetries, changing $A_{L R}$ on the order of $100 \%$ through the "running" of the coupling constants (that is, the evolution of the effective couplings as a function of the momentum transfer in the interaction) [16].

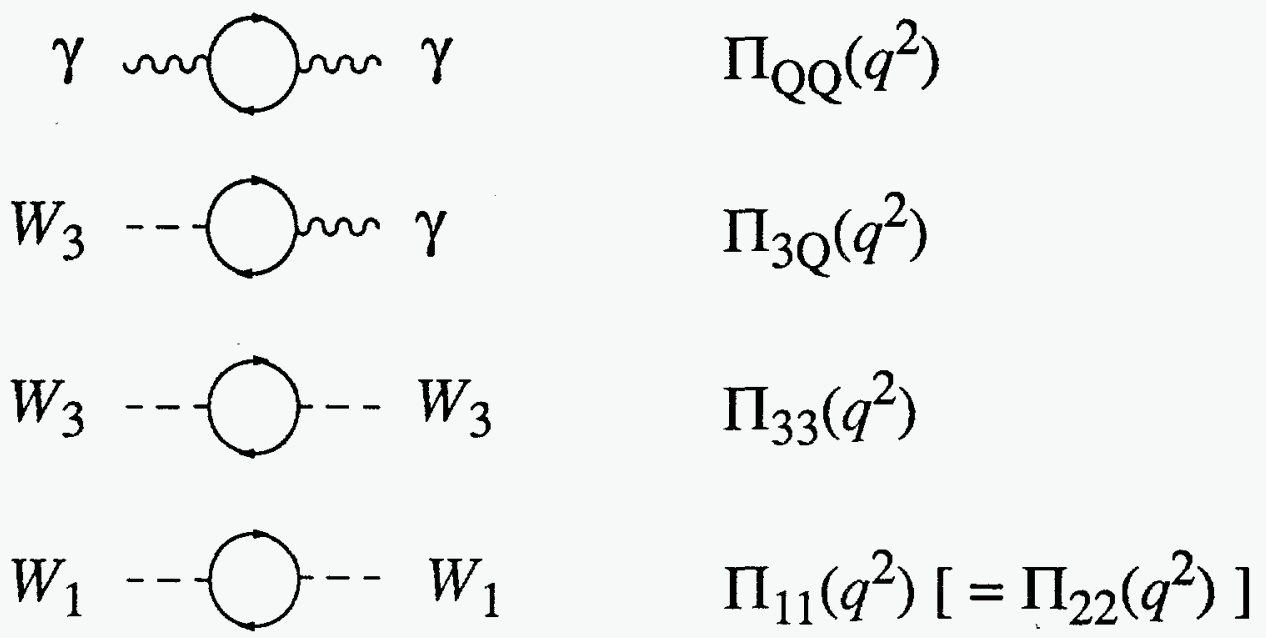

Figure 1-6: Families of oblique corrections.

\section{The S,T,U Parameterization of Oblique Corrections}

This discussion follows the review of Peskin and Takeuchi [17]. The oblique corrections can be expressed as combinations of the basic propagator correction functions (the $\Pi$ functions) shown in figure 1-6. Each $\Pi$ function depends on a loop summation over all massive particles in the theory. These functions can furthermore be expressed as Taylor expansions about $q^{2}=0$ :

$$
\begin{aligned}
& \Pi_{Q Q}\left(q^{2}\right) \approx q^{2} \Pi_{Q Q}^{\prime}(0) \\
& \Pi_{3 Q}\left(q^{2}\right) \approx q^{2} \Pi_{3 Q^{\prime}}(0) \\
& \Pi_{33}\left(q^{2}\right) \approx \Pi_{33}(0)+q^{2} \Pi_{33}(0) \\
& \Pi_{11}\left(q^{2}\right) \approx \Pi_{11}(0)+q^{2} \Pi^{\prime}{ }_{11}(0)
\end{aligned}
$$


where $\Pi^{\prime}$ is equal to $d \Pi / d q^{2}$ in these expressions. The QED Ward Identity assures that $\Pi_{Q Q}(0)=\Pi_{3 Q}(0)=0$. These expansions are valid only to the order of $\left(m_{Z} / m_{N}\right)^{2}$, where $m_{N}$ is the energy scale of any new physics phenomena.

Under this scheme the manifestations new physics through the oblique corrections are encompassed by six parameters. Three independent precision measurements, listed in table 1-2, fix three of these functions, leaving three undetermined parameters.

On their own, many of the $\Pi$ functions contain ultraviolet divergences. However, the comparison of physical observables always involves the difference of these functions. It is therefore reasonable to define the following independent combinations, which are all ultraviolet finite, as the remaining three parameters in electroweak interactions:

$$
\begin{aligned}
S & \equiv \frac{4 e^{2}}{\alpha}\left[\Pi_{33}^{\prime}(0)-\Pi_{3 Q}^{\prime}(0)\right] \\
T & \equiv \frac{e^{2}}{\alpha s^{2} c^{2} m_{Z}^{2}}\left[\Pi_{11}(0)-\Pi_{33}(0)\right] \\
U & \equiv \frac{4 e^{2}}{\alpha}\left[\Pi_{11}^{\prime}(0)-\Pi_{33}^{\prime}(0)\right]
\end{aligned}
$$

where $s$ and $c$ are the sine and cosine of the Weinberg angle.

Reference values of observables can be calculated for the Standard Model (that is, by including only Standard Model particles), given an assumption of the top quark mass $\left(m_{t}\right)$ and the Higgs mass $\left(m_{H}\right)$. It is then assumed that deviations from the model are the result of new heavy physics entering through small oblique corrections. Then the observable $O^{i}$ can be expressed as Standard Model reference value expanded to first order in $S, T$ and $U$, that is

$$
O^{i}=\left.O^{i}\left(m_{t}, m_{H}\right)\right|_{\text {Standard Model }}+a^{i} S+b^{i} T+c^{i} U
$$

where the expansion coefficients $a, b$, and $c$ are theoretically determined for each observable. Note that these coefficients are independent of $m_{l}$ and $m_{H}$. A compilation of such coefficients is found in Reference [17]. 
A general system for testing the Standard Model through oblique corrections is available through the scheme described above. It is particularly easy to discuss the case of neutral current observables (all those described in this chapter with the exception of the $W$ mass), which are independent of the $U$ parameter. Therefore, the measurement and error for a given neutral current observable defines a confidence band in $S-T$ space, with a slope equal to the ratio of the expansion coefficients, $-a^{i} / b^{i}$. The combination of measurements with different expansion coefficients then defines a region in $S-T$ space, which may be consistent with the Standard Model $(S=T=0)$, or some non-zero values of $S$ and $T$, which would be indicative of new physics. We will use this system to compare the SLD $A_{L R}$ measurement to the Standard Model in Chapter 5.
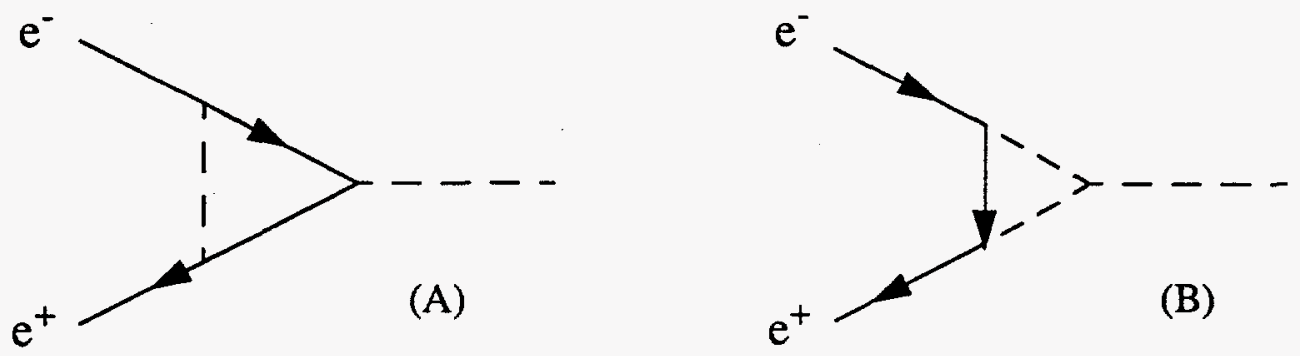

Figure 1-7: Lowest order electroweak vertex diagrams.

\subsubsection{Vertex Corrections}

Examples of vertex correction diagrams are illustrated in figure 1-7. The dashed lines can represent photons, $Z s$ or $W s$. These diagrams have an extremely small effect on the leftright asymmetry, on the order of $2 \%$ or less. Still, this level of precision is likely to become important in the near future of $A_{L R}$ measurements.

Vertex corrections are often divided into two classes: the so-called universal corrections, which are generic to all fermion species, and the non-universal corrections, which are dependent on the fermion flavor. Exactly which diagrams belong to which set is to some extent a matter of convention. Some renormalization schemes, such as that of Kennedy and 
Lynn [18], include a particular set of universal vertex corrections as well as oblique corrections in the definition of the weak mixing angle, a point that is amplified in section 1.6.
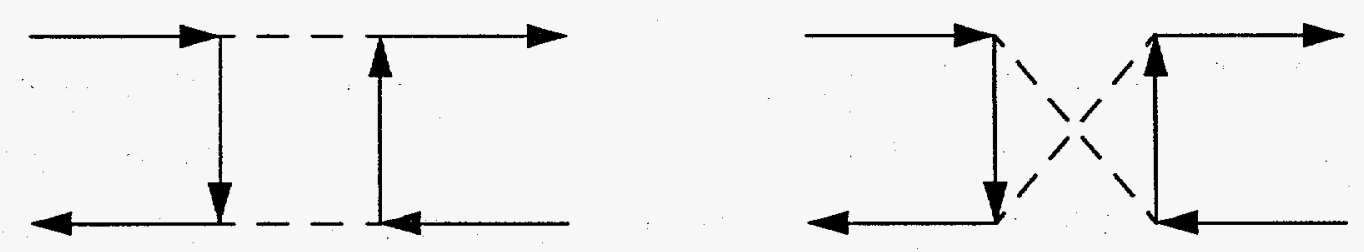

Figure 1-8: Lowest order electroweak box diagrams.

\subsubsection{Box Corrections}

Examples of box diagrams are illustrated in figure 1-8. The dashed lines can represent photons, $Z \mathrm{~s}$ or $W \mathrm{~s}$. Corrections to $A_{L R}$ arising from such diagrams are less than $0.5 \%$ and are negligible in this analysis.

\subsubsection{QCD Corrections}

All of the corrections discussed so far arise from the electroweak sector. Corrections arising from $\mathrm{QCD}$ effects are generally ignored. It has been shown that all $\mathrm{QCD}$ radiative corrections to the left-right asymmetry are suppressed to all orders by at least one factor of $\alpha$ [19]. Such corrections are therefore at least an order of magnitude smaller than electroweak corrections. Furthermore, effects arising from final state interactions and fragmentation rigorously cancel in the asymmetry, up to the level of the box diagrams. Even in box diagrams, QCD only enters in at the level of $10 \%$ (the factor of $\alpha_{\mathrm{s}}$ ) compared to the electroweak corrections.

\subsection{Properties of $A_{L R}$}

Now that we have discussed the necessary background, we describe how the left-right asymmetry is measured experimentally, and discuss the advantages of the measurement. 
In this section and throughout this document we will refer to the left-right asymmetry of some quantity $Q$ as $A_{Q}$, defined by

$$
A_{Q} \equiv \frac{\left(Q_{L}-Q_{R}\right)}{\left(Q_{L}+Q_{R}\right)}
$$

where $Q_{L}$ refers to the quantity $Q$ associated with left-handed electrons, and $Q_{R}$ refers to the quantity associated with right-handed electrons. For instance, the polarization asymmetry is the difference between the polarization of left-handed $\left(P_{L}\right)$ and right-handed $\left(P_{R}\right)$ electron bunches, normalized to the sum of those polarizations, and is denoted by $A_{P}$.

\subsubsection{The Measured Left-Right Asymmetry}

Given the form of the cross section in equation (1.23), the expected number of lefthanded $Z$ productions, $N_{L}$, and the expected number of right-handed $Z$ productions, $N_{R}$, over some period of running, $T$, is

$$
\begin{aligned}
& N_{L}=\varepsilon_{L} \sigma_{\text {unp }} \int_{0}^{T} L_{L}(t)\left[1+P_{L}(t) A_{L R}\right] d t \\
& N_{R}=\varepsilon_{R} \sigma_{\text {unp }} \int_{0}^{T} L_{R}(t)\left[1-P_{R}(t) A_{L R}\right] d t
\end{aligned}
$$

where $\varepsilon$ is the detection efficiency, a function of the detector acceptance assumed to be constant in time. $L$ is the luminosity, and $P$ is the longitudinal electron polarization, both of which change with time. The unpolarized cross section $\sigma_{\text {unp }}$ is defined in equation (1.23). If we assume that the $A_{Q}$ are zero, that is, $\varepsilon_{L}=\varepsilon_{R}, L_{L}=L_{R}=L$, and $P_{L}=P_{R}=P_{e}$, it follows from (1.41) that the measured number asymmetry, $A_{N}$, is

$$
A_{N} \equiv \frac{\left(N_{L}-N_{R}\right)}{\left(N_{L}+N_{R}\right)}=\left\langle P_{e}\right\rangle A_{L R}
$$


Corrections to the $A_{Q}=0$ assumption are discussed in Chapter 5. The luminosity-weighted average polarization $\left\langle P_{e}\right\rangle$ is defined by

$$
\left\langle P_{e}\right\rangle=\frac{\int_{0}^{T} L(t) P_{e}(t) d t}{\int_{0}^{T} L(t) d t}
$$

Throughout this document we will used the symbol $\langle Q\rangle$ to denote the luminosity-weighted time average of some quantity $Q$.

It then follows from equation (1.42) that the precision to which $A_{L R}$ is experimentally determined is given by

$$
\delta A_{L R}=\sqrt{\frac{1}{\left\langle P_{e}\right\rangle^{2} N}\left(1-\left\langle P_{e}\right\rangle^{2} A_{L R}^{2}\right)+A_{L R}^{2}\left(\frac{\delta\left\langle P_{e}\right\rangle}{\left\langle P_{e}\right\rangle}\right)^{2}}
$$

It is clear from this relation that the statistical error on $A_{L R}$ is a strong function of the electron polarization, and that the uncertainty in the polarization measurement is the dominant systematic error in the experiment.

\subsubsection{Advantages of $A_{L R}$}

A comparison of the properties of $A_{L R}$ and unpolarized asymmetry measurements is shown in table 1-3. It is clear that $A_{L R}$ carries a number of advantages. Compared to the forward-backward asymmetry for leptons, it is a relatively large asymmetry with a higher sensitivity to the weak mixing angle. Compared to the forward-backward asymmetry for $b$ quarks and the tau polarization measurement, $A_{L R}$ is statistically advantageous, making use of a larger fraction of the data sample. Additionally, when compared to the other measurements, $A_{L R}$ is very clean in terms of systematic effects arising from backgrounds, acceptance, efficiency, and mixing. 


\begin{tabular}{|c|c|c|c|c|}
\hline Property & $A_{L R}^{\text {meas }}$ & $A_{F B}^{l}$ & $A_{F B}^{b}$ & $P_{\tau}$ \\
\hline \hline Asymmetry & $0.13 \cdot \mathrm{P}_{\mathrm{e}}$ & 0.013 & 0.091 & 0.13 \\
\hline Sensitivity to $\sin ^{2} \theta_{W}^{e f f}$ & $7.9 \cdot \mathrm{P}_{\mathrm{e}}$ & 1.5 & 5.6 & 7.9 \\
\hline Fraction of usable $Z$ decays & 0.96 & 0.12 & 0.19 & 0.04 \\
\hline efficiency acceptance & 0.90 & $0.7-0.8$ & 0.10 & 0.30 \\
\hline Systematic Errors & & & & no \\
\hline \hline beam polarization & yes & no & no & yes \\
\hline efficiency acceptance & no & yes & yes & yes \\
\hline backgrounds & no & no & yes & no \\
\hline B mixing & no & no & yes & $2 \%$ \\
\hline electroweak interference & $2 \%$ & $100 \%$ & $5 \%$ & no \\
\hline
\end{tabular}

Table 1-3: Properties of various electroweak asymmetries.

\subsection{The Weak Mixing Angle}

It is desirable to compare various electroweak asymmetry measurements by converting the observations into a value of a single electroweak parameter. The sine-squared of the weak mixing angle has become the standard of choice over the years; however, there are many definitions of $\sin ^{2} \theta_{W}$, and one must be careful when making such comparisons.

As a reminder, we point out that the fundamental, or "bare" value of $\sin ^{2} \theta_{W}$ is defined in terms of the gauge coupling constants, as illustrated in figure 1-2. Thus the bare quantity, denoted as $s^{2}$, is given by

$$
s^{2} \equiv \frac{g^{\prime^{2}}}{g^{\prime^{2}+g^{2}}}, \quad c^{2}=1-s^{2}
$$

In the same way that we cannot measure the bare electric charge experimentally, neither can we directly measure the bare Weinberg angle. 


\subsection{1 $s_{*}^{2}$}

It has been shown that the effects of the oblique corrections and a particularly defined set of universal vertex corrections can be absorbed into the coupling constants and propagators of the electroweak matrix elements, without altering the form of the interactions themselves [18]. In this case, the neutral current Lagrangian is given by

$$
\mathcal{L}_{\text {neutral }}=e_{*} \bar{f} \gamma^{\mu} f A_{\mu}-\frac{e_{*}}{s_{*} c_{*}} \bar{f} \gamma^{\mu}\left[\left(1-\gamma_{5}\right) T_{f}^{3}-s_{*}^{2} Q_{f}\right] f Z_{\mu}
$$

from which it follows that the left-right asymmetry, now corrected for all orders of vacuum polarization, is

$$
A_{L R}\left(q^{2}\right)=\frac{2\left[1-4 s_{*}^{2}\left(q^{2}\right)\right]}{1+\left[1-4 s_{*}^{2}\left(q^{2}\right)\right]^{2}} .
$$

This quantity is essentially what is directly measured by experiment, to within the small non-universal vertex corrections and box corrections.

\subsection{2 $s_{s}^{2}$ and $s_{o}^{2}$}

One definition of the electroweak mixing angle in terms of measured quantities is suggested by Sirlin [20]:

$$
s_{s}^{2} \equiv 1-\left(\frac{M_{W}}{M_{Z}}\right)^{2}
$$

This quantity has the disadvantage in being related to the $W$ mass, which is not one of the best-measured electroweak parameters. The currently most precise measurement [21] determines the Sirlin angle to be

$$
s_{s}^{2}=0.2256 \pm 0.0047
$$


A more accurate determination of the mixing angle is suggested by [22]

$$
\left(\sin 2 \theta_{W}\right)_{o}^{2}=\left[\frac{4 \pi \alpha_{*_{0}}\left(m_{Z}^{2}\right)}{\sqrt{2} G_{F} m_{Z}^{2}}\right]=s_{o}^{2}\left(1-s_{o}^{2}\right)
$$

where $\alpha_{*_{0}}$ is the running electromagnetic coupling, renormalized by oblique corrections to the photon propagator, accounting only for known quarks and leptons. This value is calculated to be [23]

$$
\alpha_{*_{o}}\left(m_{Z}^{2}\right)^{-1}=128.80 \pm 0.12
$$

which, when combined with the values given in table 1-2, yields a value

$$
s_{o}^{2}=0.23135 \pm 0.00031
$$

This extremely accurate reference value may now serve as a basis for detecting new heavy physics through oblique radiative corrections. Of particular interest:

$$
s_{*}^{2}-s_{o}^{2}=\left.\left(s_{*}^{2}-s_{o}^{2}\right)\right|_{\text {Standard Model }}+\frac{\alpha}{c^{2}-s^{2}}\left[\frac{1}{4} S-s^{2} c^{2} T\right]
$$

\subsection{3 $\sin ^{2} \theta_{W}^{\text {eff }}$}

The most logical choice of definition for experimentalists is the effective weak mixing angle, $\sin ^{2} \theta_{W}^{\text {eff }}$. The effective mixing angle is strictly defined at the $Z$ pole, such that

$$
A_{L R}^{0}=A_{L R}\left(m_{Z}^{2}\right) \equiv \frac{2\left[1-4 \sin ^{2} \theta_{W}^{\mathrm{eff}}\right]}{1+\left[1-4 \sin ^{2} \theta_{W}^{\mathrm{eff}}\right]^{2}}
$$

and

$$
\sin ^{2} \theta_{W}^{\text {eff }} \equiv \frac{1}{4}\left(1-\frac{v_{e}^{\text {eff }}}{a_{e}^{\text {eff }}}\right)
$$


where the electron weak coupling constants $v_{e}$ and $a_{e}$ have been renormalized to the energy scale of the $Z$ mass to from effective coupling constants. The effective mixing angle implicitly includes the oblique corrections and universal vertex corrections, as well as the nonuniversal electron vertex corrections. The effects of initial state radiation are not and cannot be included in a general definition, because they depend on detector acceptance. Therefore each experiment must account for initial state radiation, and the electroweak interference that accompanies a real measurement away from the $Z$ pole, when converting an observation into a value of $\sin ^{2} \theta_{W}^{\text {eff }}$.

The relationship between the effective mixing angle and other mixing angle definitions is made explicit in the following equations:

$$
\begin{aligned}
\sin ^{2} \theta_{W}^{\text {eff }} & =\left(1+\Delta \kappa_{e}\right) s_{s}^{2}\left(m_{Z}^{2}\right) \\
& =\left(1+\Delta \kappa^{\text {univ }}+\Delta \kappa_{e}^{\text {non-univ }}\right) s_{s}^{2}\left(m_{Z}^{2}\right) \\
& =\left(1+\Delta \kappa_{e}^{\text {non-univ }}\right) s_{*}^{2}\left(m_{Z}^{2}\right)
\end{aligned}
$$

where $\Delta \mathrm{\kappa}_{e}$ is the weak form factor associated with the electron/positron vertex which parameterizes the difference between $s_{s}^{2}$ and $\sin ^{2} \theta_{W}^{\text {eff }}$. This form factor is calculated to have a Standard Model value of $\Delta \kappa_{e}=0.0226$ [24]. It can be further broken down into universal and non-universal parts, $\Delta \kappa^{\text {univ }}$ and $\Delta \kappa^{\text {non-univ }}$. As discussed in the previous section, the universal part is absorbed into the definition of $s_{*}^{2}$. The remaining non-universal part is small, with a calculated Standard Model value of $\Delta \kappa^{\text {non-univ }} \approx 0.003$ [25]. Hence the value of $\sin ^{2} \theta_{W}^{\text {eff }}$ is substantially different from that of $s_{s}^{2}$ but very close to that of $s_{*}^{2}$.

\subsubsection{Converting to the Weinberg Angle}

The machinery used to convert a measurement of $A_{L R}$ into $\sin ^{2} \theta_{W}^{\text {eff }}$ is the software package ZFITTER [24]. The result of many man-years of effort, ZFITTER is a flexible program that calculates Standard Model cross sections and asymmetries by various methods. The primary method employs analytic formulae with higher order corrections, including the oblique, vertex, and box diagram corrections discussed above, to first order. This is the 
method used for the Standard Model fits presented in this document. Other methods use model-independent approaches based on effective couplings or partial decay widths.

In addition, ZFITTER includes the effects of initial state radiation, either employing no cuts on the bremsstrahlung photon phase space, or a cut on the maximum allowed energy of a bremsstrahlung photon, or simultaneous cuts on the energy and acollinearity of the final state fermions.

\subsection{Thesis Outline}

The remainder of this thesis deals with the experimental aspects of the $A_{L R}$ measurement.

Chapter 2 describes the hardware necessary to create, transport, and collide polarized electrons and positrons, as well as the hardware components of the Møller and Compton polarimeters and the SLD detector.

Chapter 3 concentrates the details of Compton scattering kinematics, the Compton polarimeter analysis and associated systematic errors, and presents the resulting average luminosity-weighted polarization.

Chapter 4 describes the analysis of SLD data, the event selection criteria, and the background estimation, and gives the result for the measured $Z$ asymmetry.

Chapter 5 discusses the corrections to the measured polarizations and asymmetries (in particular the chromaticity correction) and the various systematic cross-checks that were made, and gives the result for $A_{L R}$ and the associated estimate of systematic errors. This result is then converted into a measurement of $\sin ^{2} \theta_{W}^{\text {eff }}$ and compared to other experiments and the Standard Model. 


\section{Chapter 2}

\section{Experimental Apparatus}

Given the definition of $A_{L R}$, it is clear that we require certain experimental apparatus in order to carry out the measurement, specifically:

- A source of polarized electrons

- An electron accelerator and spin transporter

- A beam polarization monitor

- $\mathrm{A} Z$ boson detector

The fulfillment of these requirements is met in turn by the SLAC Linear Collider (SLC), the Møller and Compton polarimeters, and the SLD detector. The hardware components and general operation of the SLC, the SLD detector, and the polarimeters are the subjects of this chapter.

\subsection{The Polarized SLC}

The SLAC Linear Collider is the world's first linear $\mathrm{e}^{+} \mathrm{e}^{-}$collider, producing head-on collisions of electrons and positrons accelerated in the SLAC linear accelerator (or linac). These collisions result in the production of massive resonant particles such as the $Z$ boson. The SLC is also the first and only accelerator to produce $Z$ bosons with polarized electron beams [26]. 


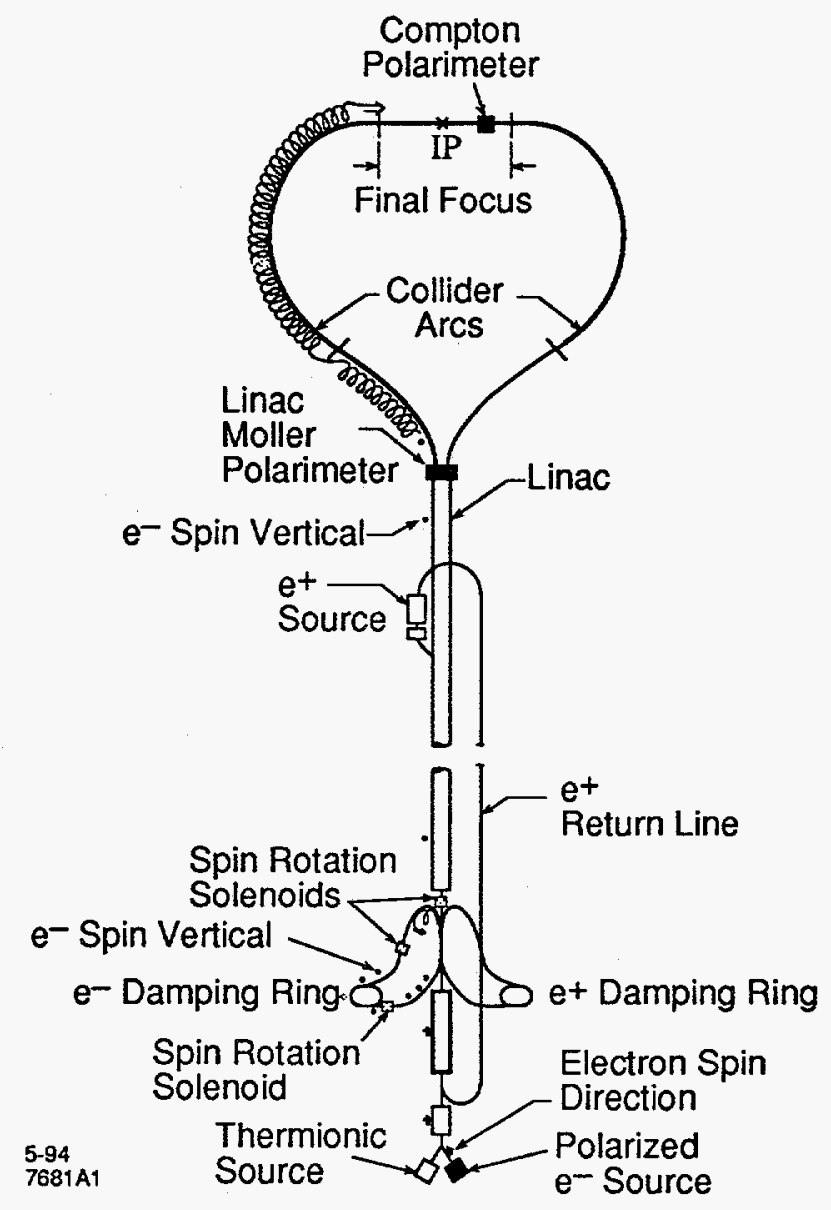

Figure 2-1: The Polarized SLC

The following brief overview of SLC operation refers to figure 2-1. Detailed discussions of SLC components appear in later sections.

Longitudinally polarized electrons are created by photoemission from a strained gallium arsenide cathode in the polarized electron source and injected into the linac. The electrons are diverted into the north damping ring, where they are stored for one machine cycle $(8.3 \mathrm{~ms})$ with vertical spin orientation. Electrons are then extracted from the damping ring and accelerated in the linac to approximately $46 \mathrm{GeV}$. A Møller polarimeter at the end of the linac may be intrusively inserted for diagnostic polarization measurements at this point. Otherwise, electrons are bent in the north arc and undergo some spin precession and polarization loss before being focussed in the final focus region and colliding with the 
positron bunch at the SLD interaction point. Collision products are measured by the SLD detector. The electron bunch then continues past the interaction point and collides with the Compton polarimeter polarized target. The products from this collision are scattered in to the Compton Cherenkov detector, which provides a continuous monitor of the longitudinal electron polarization. The main electron bunch continues down the arc, where it finally encounters the energy spectrometer magnets before entering the electron dump. Synchrotron photons from the spectrometer magnets are detected in the Wire Imaging Synchrotron Radiation Detector, which provides a continuous precision beam energy measurement.

\subsubsection{Polarized Electron Source}

Polarized electron beams have been in use at SLAC since the spring of 1992. The SLAC Linear Collider injector requires that two 2 ns pulses of $4.5-5.5 \times 10^{10}$ electrons, separated by $61 \mathrm{~ns}$, to be produced at $120 \mathrm{~Hz}$ [27]. These specifications are met by the Polarized Electron Source (PES), which consists of a diode-type electron gun in which electrons are extracted from a gallium-arsenide photocathode by a laser operating near the semiconductor band gap energy. A schematic representation of the PES is shown in figure 2-2. A discussion of the main PES components follows.

\section{Gallium-Arsenide (GaAs) Photocathodes}

It has been long known that polarized electrons can be extracted by photoemission from a semiconductor surface [28]. Figure 2-3 shows an energy level diagram for gallium-arsenide. The solid lines indicate transitions induced by right-handed photons and the dotted lines indicate transitions induced by left-handed photons. The numbers in circles are the relative Clebsh-Gordon coefficients for each transition.

From this diagram, we see that photons with an energy $1.52 \mathrm{eV} \leq E_{\gamma} \leq 1.86 \mathrm{eV}$ only excite transitions from the $P_{3 / 2}$ level of the valence band. If we further assume that the photons are right-handed (that is to say, they have positive helicity, or their angular momentum and momentum are parallel), only two transitions are possible: the $P$ state with $m_{j}=-3 / 2$ can make a transition to the $S$ state with $m_{j}=-1 / 2$, and the $P$ state with $m_{j}=-1 / 2$ can make a transition to the $S$ state with $m_{j}=+1 / 2$. The former transition ejects an electron with spin 


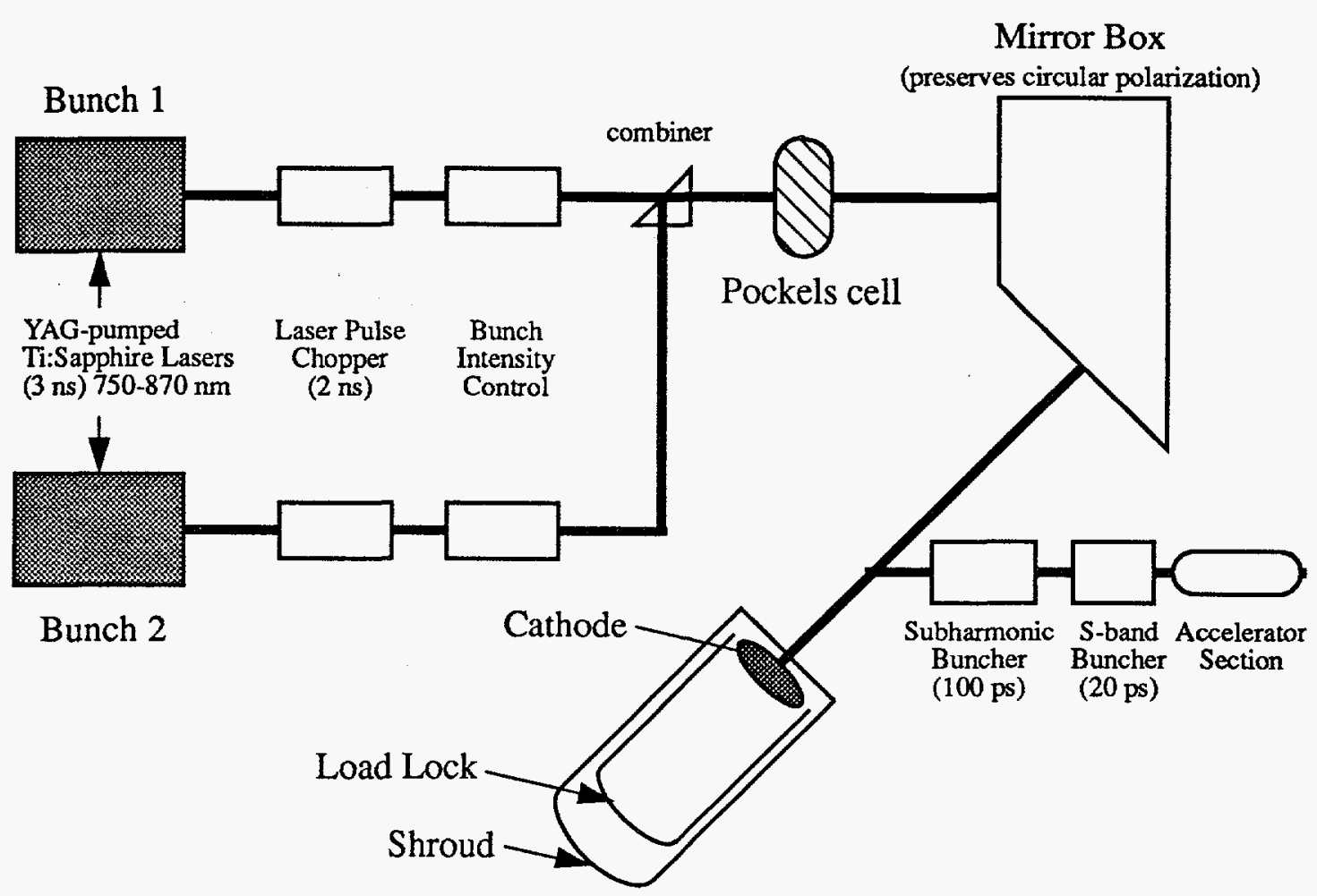

Figure 2-2: The SLC polarized electron source
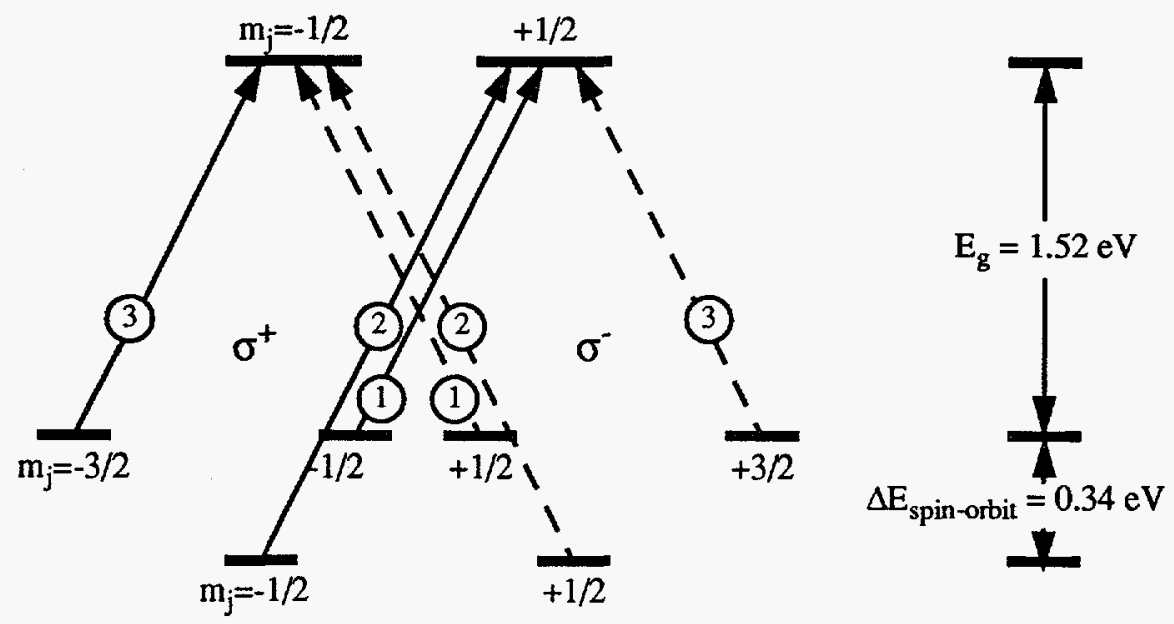

Figure 2-3: Energy level diagram for a Gallium Arsenide photocathode 
antiparallel to the incident photon direction (that is, parallel to its ejected momentum, or right-handed), and has a probability three times greater than the latter transition, which ejects left-handed electrons. In other words, the absorption of right-handed photons produces a preferentially right-handed electron bunch, with a maximum polarization given by

$$
P_{\max }=\frac{3-1}{3+1}=50 \%
$$

The symmetry of the energy levels shows that left-handed photons produces a bunch of lefthanded electrons with the same polarization.

Of course, all that has been shown at this point the that we can create polarized electrons in the conduction band of GaAs. In normal GaAs, the energy gap between the conduction band and the free electron state (referred to as the work function of the material) is on the order of $2.5 \mathrm{eV}$, and even under the large electric fields applied in the gun, pure GaAs is a poor photoemitter.

Photoemission quality is quantified by the quantum efficiency or $Q E$ of a material, which is the probability that an electron will be emitted when a photon is incident on the material surface.

It turns out that a surface application of cesium serves to bring the work function to zero or even negative, vastly increasing the $Q E$ of the cathode. During normal source operation, in order to activate the highest quantum efficiency, the cathode is heated to $610^{\circ} \mathrm{C}$ for one hour, cooled to room temperature, and then treated with Cs until the photocurrent peaks. To fix the cycle, the cathode is treated with a codeposition of $\mathrm{Cs}$ and $\mathrm{NF}_{3}$.

We have seen that in normal Gallium arsenide, the energy degeneracy in the $P_{3 / 2}$ level makes $50 \%$ the theoretical upper maximum achievable polarization. If there were some way to break this degeneracy, then the theoretical maximum polarization would be $100 \%$. It turns out that the use of a strained lattice cathode, added to the polarized source in 1993 and one of the keys to the success of the run, does just that. 


\section{Strained GaAs Cathodes}

The SLC strained-lattice cathode was manufactured by growing a thin layer of gallium arsenide on a gallium arsenide phosphide substrate. The substrate layer has a smaller lattice spacing constant than the gallium arsenide, and the resulting mismatch breaks the $P_{3 / 2}$ degeneracy in the valence band. The 1993 cathode consists of a 0.3 micron layer of GaAs on a 2.5 micron substrate. The resulting energy level diagram is shown in figure 2-4.
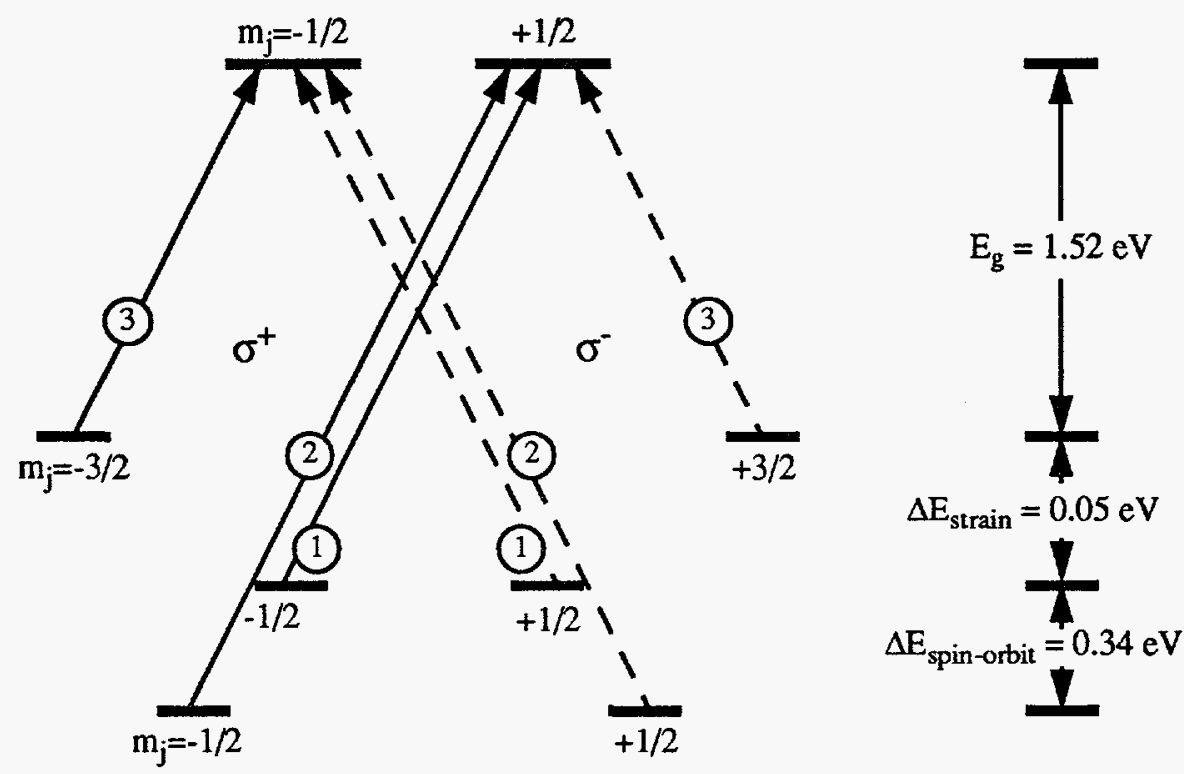

Figure 2-4: Energy level diagram for a strained Gallium Arsenide photocathode

The energy difference between the $m_{j}= \pm 3 / 2$ and $m_{j}= \pm 1 / 2$ levels in the $P$ state is very small, $\Delta \mathrm{E}_{\text {strain }}=0.05 \mathrm{eV}$. Because this value is so small, and because it is a challenge to grow crystals wherein the strain is evenly distributed, it is difficult to achieve the theoretical maximum polarization. Also, the extracted electron polarization is a sensitive function of the photon wavelength. Nevertheless, polarizations substantially higher than $50 \%$ are obtained. Figure 2-5 shows measurements of various cathode types. Bulk gallium arsenide was the material used in the 1992 run.

Unfortunately, this data cannot be taken as a direct measure of the source polarization $P_{e}^{\text {source }}$, because the Mott Polarimeter used for the measurement was calibrated with a 


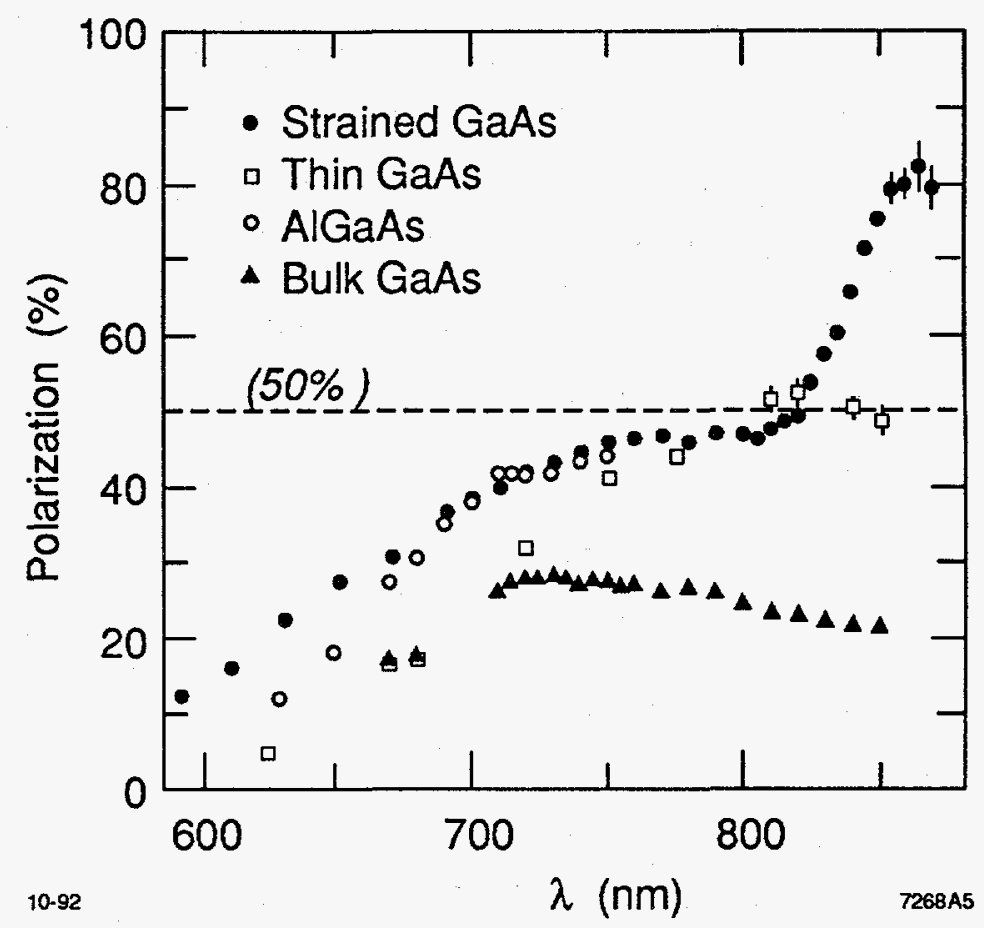

Figure 2-5: Measurements of various photocathode materials

Møller polarimeter, which is now known to have scale errors associated with atomic binding energy effects in the Møller target [29]. Nevertheless, the dramatic increase in the polarization yield as a result of the strained cathode is evident. A new cathode installed in 1994 has already been shown to produce polarization at the level of $80 \%$, measured by the well-calibrated Compton polarimeter, so SLC/SLD can look forward to even more statistical power in future running.

\section{The PES Light Source (PLS)}

In 1992, a flashlamp pumped dye laser was used to illuminate the bulk $\mathrm{GaAs}$ photocathode. However, the use of strained GaAs cathodes requires higher pulse energies (greater than $50 \mu \mathrm{J}$ ) because the thickness of the strained layer is actually less than the penetration depth for the operating photon energies, and quantum efficiency is thereby inherently lower. Strained GaAs cathodes also require longer wavelengths for optimal polarization. 
Referring to figures 2-3 and 2-4 we see that a bulk GaAs can be illuminated by energies as high as $1.86 \mathrm{eV}$, or wavelengths as short as $667 \mathrm{~nm}$, whereas strained GaAs cannot go higher than $1.57 \mathrm{eV}$, or lower than $790 \mathrm{~nm}$. There were in fact no commercial lasers available with the required specifications, so the necessary system was developed at SLAC.

The only commercially available solid state laser material which operates over the required wavelength range at the required power and repetition rate is titanium-doped sapphire $\left(\mathrm{TI}^{+3}: \mathrm{Al}_{2} \mathrm{O}_{3}\right)$, and the $1993 \mathrm{PLS}$ was designed around this material. Two Ti-sapphire resonant cavities, each operating at $120 \mathrm{~Hz}$, are both pumped by two frequency doubled Nd:YAG lasers, each operating at $60 \mathrm{~Hz}$. The first Ti-sapphire laser runs is at $864 \mathrm{~nm}$ as extracts electrons for collision. The second runs at $707 \mathrm{~nm}$ and extracts electrons for the positron source. A complex feedback system reduces the output jitter of the lasers to less than $3 \%$ RMS [27].

Both beams pass through the circularly polarizing Pockels cell at the same voltage setting. A Pockels cell consists of a $\mathrm{KD}^{*} \mathrm{P}^{1}$ crystal sandwiched between two plates that generate an electric field along the longitudinal (or $\mathrm{z}$ ) axis of the crystal. When light passes through the crystal, a phase shift is generated between field components projected along the fast ( $\mathrm{x}$ ) axis and the slow (y) axis. This phase shift is proportional to the voltage applied to the plates. Thus the Pockels cell acts as a variable phase shift generator. A given Pockels cell has a unique voltage at which it operates as a quarter wave plate, called the quarterwave voltage (QWV), for a given frequency of light. When $+\mathrm{QWV}$ is applied to the source Pockels cell, the source laser is circularly polarized with one handedness. When -QWV is applied to the cell, the source laser is circularly polarized with the opposite handedness (which handedness is created depends on the cell alignment and must be calibrated experimentally).

The sign of the source Pockels cell QWV is chosen randomly on a pulse-by-pulse basis, which controls the helicity of the source laser, which in turn controls the helicity of the extracted electrons. Thus the helicity of the beam pulse is chosen randomly, and this information is transmitted and incorporated into the SLD data acquisition. The helicity bits are

1. Potassium Dideuterium Phosphate 
transmitted on three redundant systems: the KVM (Klystron Veto Module) system, the Mach line (direct signal wires from the PLS to SLD), and the PMON (Polarization MONitor) system. The helicity bit transmission has been rigorously tested (see section 5.3).

Higher currents are required in the second electron bunch, in order to extract enough positrons from the positron source to match the first electron bunch. For this reason the second Ti-sapphire laser operates at a lower wavelength, in order to increase the quantum efficiency and avoid the charge limit effect (see below). Therefore, the $n$th machine pulse (and $n$th PLS Pockels cell voltage setting) has two electron pulses associated with it, a polarized bunch for collisions, and a bunch for the positron source, with a low but unknown polarization. Here is the important point: even if the second electron bunch retains some polarization information, and if this information were somehow imparted to the positrons created by this bunch, this would still have no effect on the $A_{L R}$ experiment. This is because the positrons extracted by the $n$th unpolarized electron bunch are used to collide with the $(n+1)$ th polarized electron bunch whose polarization state is completely uncorrelated with the $n$th bunch (since the polarization of any bunch is chosen randomly). Therefore, any effect stemming from residual positron polarization of this type rigorously vanishes when averaged over time.

\section{The Electron Gun}

The SLC polarized electron gun, shown in figure 2-6, employs a conventional diode design. Dúring operation, the cathode potential is $-120 \mathrm{kV}$, which draws a space-charge limited current of 8.9 amperes, or $1.1 \times 10^{11}$ electrons in a $2 \mathrm{~ns}$ bunch. However, as discussed in the next section, this is not the limiting factor on current from the PES.

The high voltage end of the gun is connected to the so-called load lock, which allows a new photocathode to be installed in the gun without affecting the gun's ultra-high vacuum [30]. The load lock system provides three major advantages over the previous system. First, without the load lock system, the gun must be brought to atmosphere in order to change cathodes, and then re-baked each time with the cathode in place. This degrades both the high voltage performance of the gun and the quantum efficiency of the cathode. The second advantage of the load lock system is that the time required to load a new photocathode is 


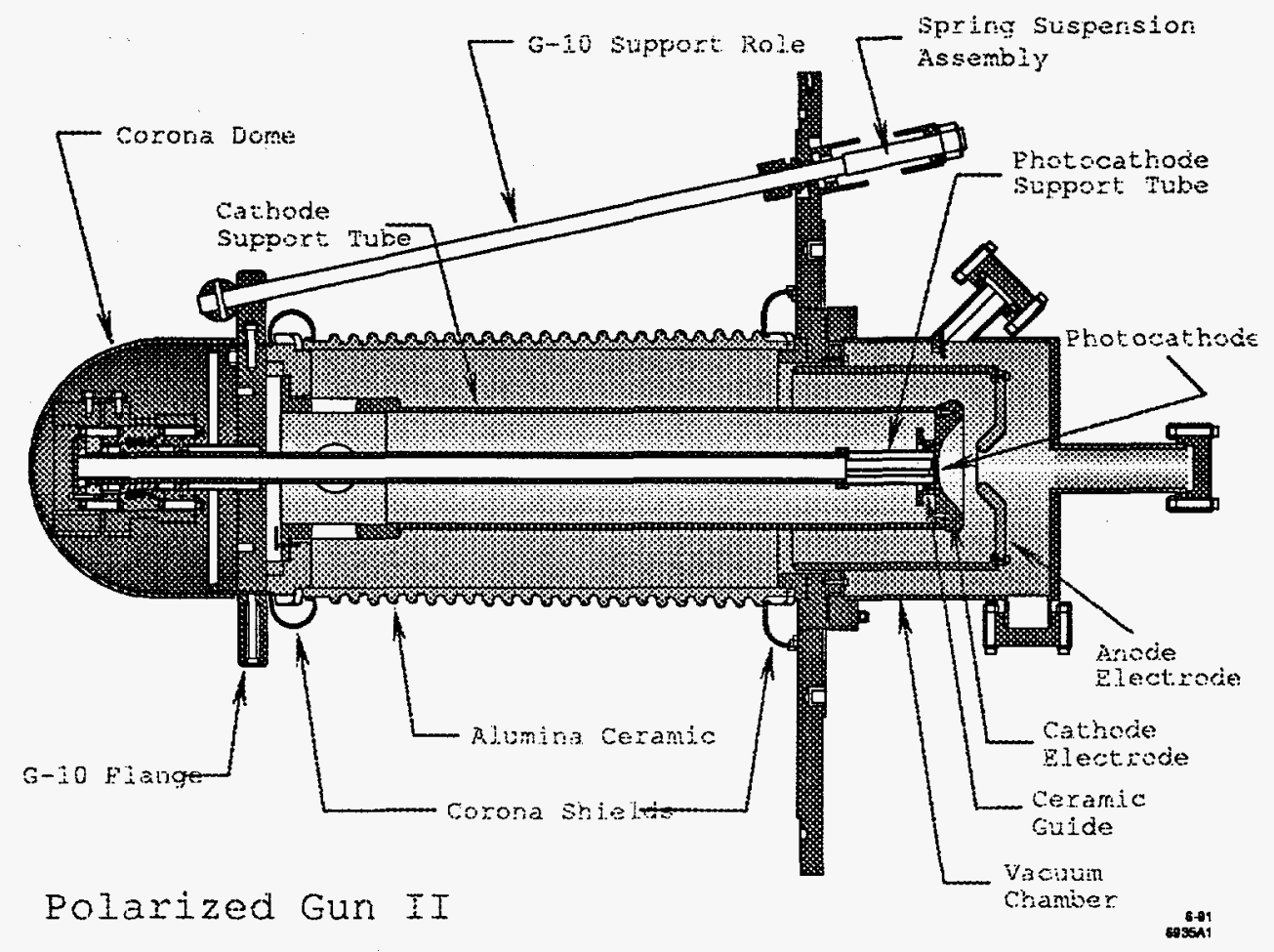

Figure 2-6: The SLC Polarized Electron Gun

reduced from three weeks to a matter of hours. Finally, the cathode is easily removed for high voltage processing of the gun electrodes.

\section{The Charge Limit}

For low source laser currents, the number of electrons extracted from the source is proportional to the laser pulse energy. However, the dependence becomes strongly non-linear at higher wavelengths and pulse energies, and the number of extracted electrons eventually saturates around $7 \times 10^{10}$ electrons per pulse. This number is well below the inherent space charge limit of the gun, which is estimated at $1.1 \times 10^{11}$ electrons per pulse [31].

The exact cause of the charge limit effect has yet to be determined, although it is hypothesized to be a result of charge trapping at the cathode surface. However, the effect carries a hidden advantage: by operating in the charge limit regime of the photocathode response, effects arising from intensity jitter in the electron beam are minimized. 


\subsubsection{Flat Beam Operation}

The SLC was designed to operate with round beams, in which the horizontal and vertical beam emittance are equal $\left(\varepsilon_{\mathrm{x}}=\varepsilon_{\mathrm{y}}\right)$. This mode of operation facilitates optical matching in beam lines with cross-plane coupling elements such as the solenoidal spin rotator magnets and the SLC arcs. However, storage rings naturally produce flat beams, in which $\varepsilon_{\mathrm{x}} \gg$ $\varepsilon_{y}$, and the collision of flat beams produces higher luminosity.

Tests in 1992 demonstrated the technical feasibility of flat beam operation in the SLC [32]. A number of new accelerator techniques were required [33], not the least of which was a new method of spin rotation, which is discussed in section 2.1.4. Flat beam operation was begun early in the 1993 run, and within a short time the peak luminosity of the previous running cycle was reached and then surpassed. Figure 2-7 shows the time history of SLC luminosity for 1991 through 1993 . The increase in 1993 is due primarily to the success of flat beam operation. The integrated luminosity for 1993 was approximately $1.77 \mathrm{pb}^{-1}$ [34].

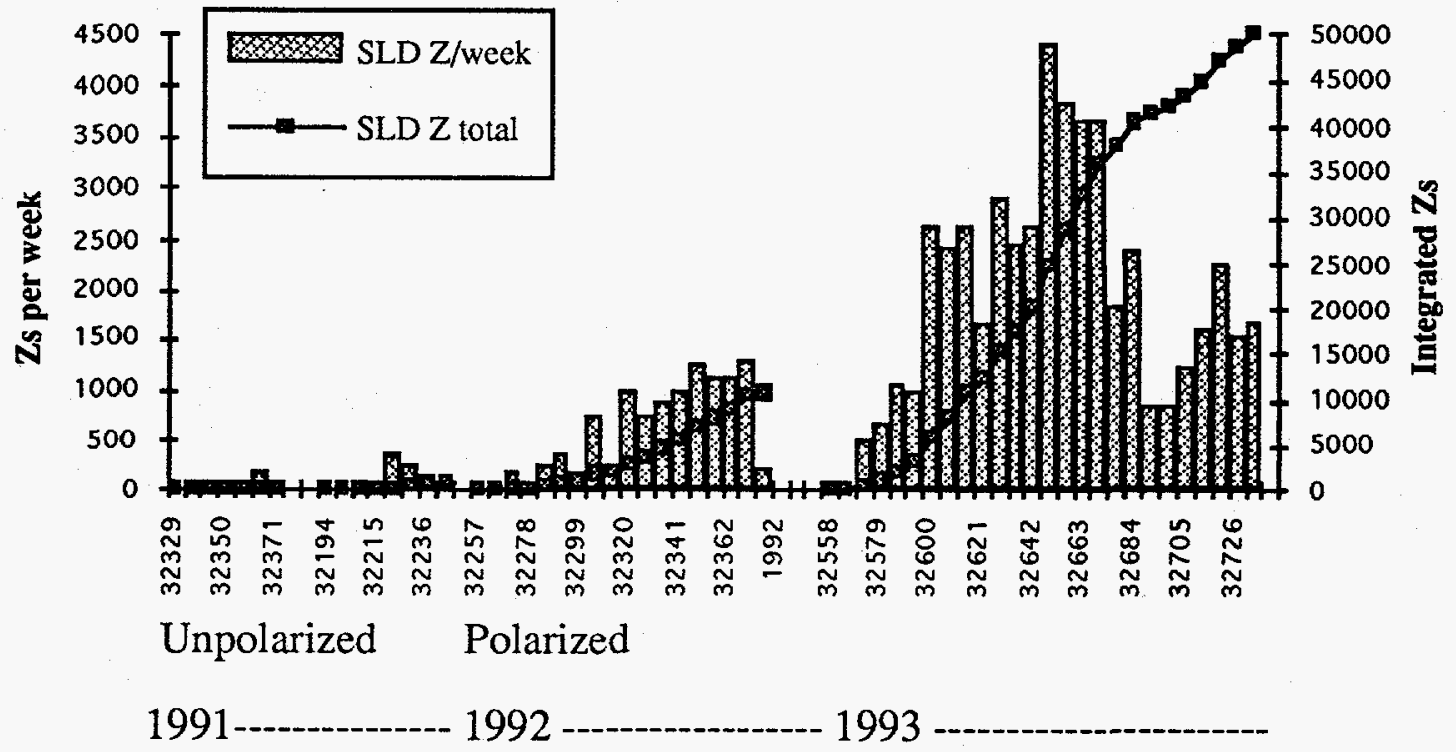

Figure 2-7: The time history of SLC luminosity. 


\subsubsection{Spin Dynamics}

It is of course not sufficient merely to have a source of polarized electrons. We also require a method of maintaining and manipulating the spin in the accelerator. In order to describe spin transport in the SLC, we must first discuss some general principles of electron spin dynamics.

The precession of electron spins in magnetic fields is described fully by the BMT equations [35]. In the limit of planar motion through transverse magnetic fields, the spin precession angle of the electrons, $\theta_{s}$, is directly related to the momentum precession angle of the electrons, $\theta_{b}$, by the following equation

$$
\theta_{s}=\gamma\left(\frac{g-2}{2}\right) \theta_{b}
$$

where $(g-2) / 2=1.163 \times 10^{-3}$ is the anomalous magnetic moment of the electron, and $\gamma$ is the Lorentz factor, which is equal to $E_{b} / m_{e}$ in the limit of large beam energy, $E_{b}$. The combination of these two coefficients is known as the spin tune, $(v)$ which has a value on the order of 100 at the full SLC energy.

In the case of solenoidal magnetic fields, the spin precession angle is given by

$$
\theta_{s}=\frac{e L(\vec{B} \times \hat{s})}{m_{e} c \gamma}
$$

where $L$ is the length of the magnet, $B$ is the solenoidal field strength and $\hat{s}$ is the spin direction unit vector. Note that a solenoidal field precesses only transverse spin components.

\subsubsection{Spin Transport}

The main experimental goal regarding spin transport in the SLC is to maximize the longitudinal polarization at the SLD interaction point. This translates into three SLC operational goals. First, spins must be oriented vertically in the North Damping Ring in order to prevent loss of polarization. Second, spin orientation must be controlled at the IP. Finally, spin diffusion (defined below) in the North Arc must be minimized. 


\section{The North Damping Ring}

Horizontal spin components are randomized in the damping ring, where small energy differences are magnified hundreds of times by the spin precession about the vertical axis. Therefore it is essential that the spin vector be vertical when electrons enter the damping ring. Polarized electrons are extracted from the source cathode with a longitudinal polarization $P_{e}$ source and injected into the linac (point 1 on figure 2-8). The electrons have an energy of $1.19 \mathrm{GeV}$ when they enter the linac-to-ring (LTR) extraction line. The bend angle of the LTR section precesses the electron spins according to equation (2.2). This bend angle was chosen to rotate the spin vector exactly $540^{\circ}$, for the design energy of $1.21 \mathrm{GeV}$. However, this energy mismatch only results in a $0.8 \%$ loss of polarization. For all practical purposes, the bend rotates the longitudinal spin vector $[0,0,1]$ into a transverse spin vector $[1,0,0]$. After this point (point 2 on the figure), the electrons pass through the LTR solenoid which rotates the spin vector into the vertical orientation $[0,1,0]$ (point 3 ), as dictated by equation (2.3).

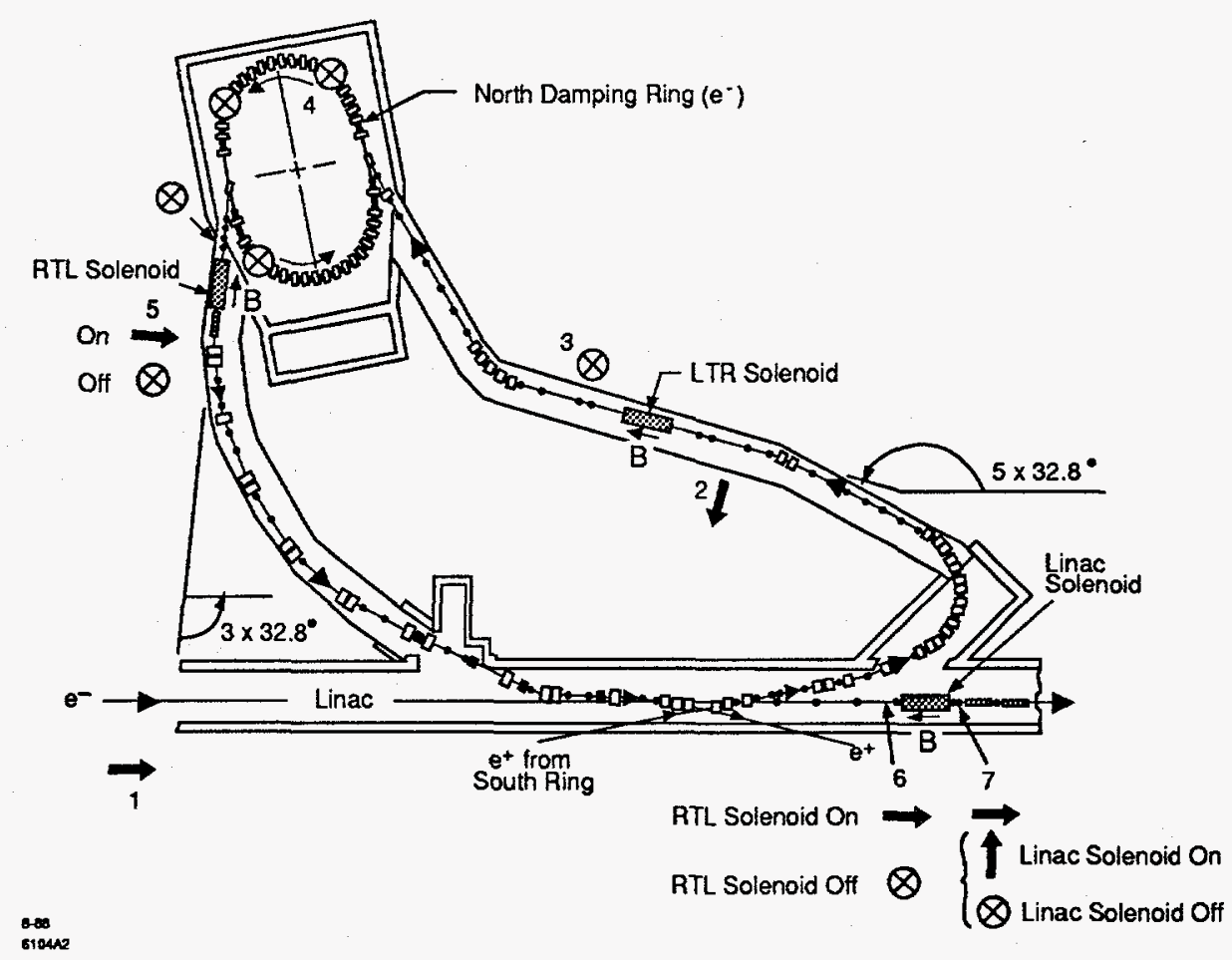

Figure 2-8: Spin rotation in the North Damping Ring 
After being cooled (cooling is the process of reducing the energy spread of the electron bunch through synchrotron radiation damping) for $8.3 \mathrm{~ms}$, the electrons are extracted from the damping ring with the flat beam profile $\left(\varepsilon_{\mathrm{x}} \approx 9 \cdot \varepsilon_{\mathrm{y}}\right)$ and accelerated down the linac to their full energy ( $46 \mathrm{GeV}$ ). The RTL and linac solenoids are turned off during normal running, in order to avoid rotating the flat beam profile, and the spin vectors remain in the vertical orientation. At the end of the linac, the bunch has a transverse polarization denoted by $P_{e}^{\text {linac }}$, which differs from $P_{e}^{\text {source }}$ by less than $1.0 \%$.

\section{Spin Rotation}

A spin rotation system is required to manipulate the spin orientation from vertical at the end of the linac to longitudinal at the IP. A general spin rotation system must induce controlled rotations about two orthogonal axes. One possible implementation of such a system is shown in figure 2-9.

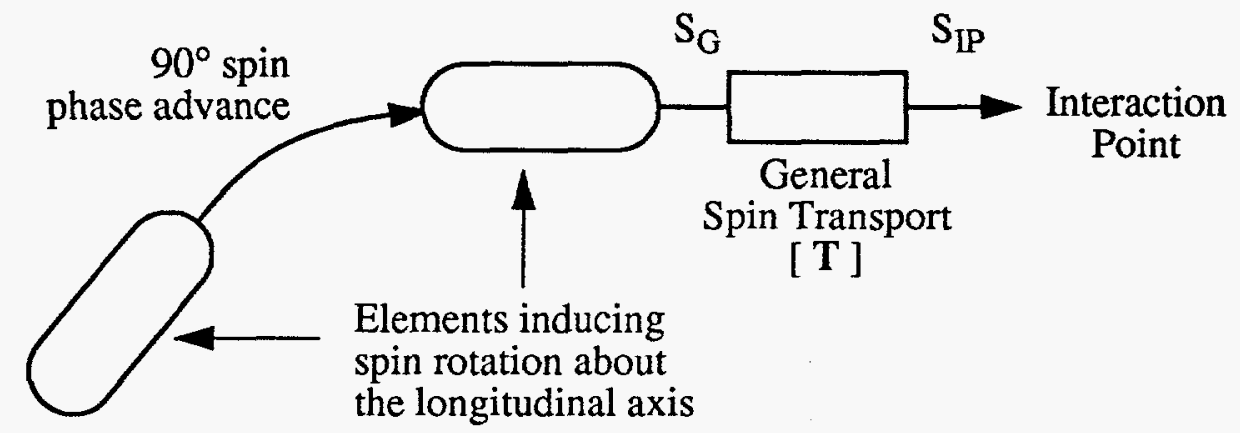

Figure 2-9: General spin rotation system

The purpose of the general spin rotation system is to set the spin vector at the interaction point, $S_{I P}$, equal to $(0,0,1)$, that is, to a purely longitudinal spin state. This involves setting the spin at some point in the machine, $S_{G}$, such that the spin precession in the rest of the machine, characterized by the transport matrix $T$, produces the desired $S_{I P}$. If one can make two arbitrary rotations about two orthogonal axes, one can satisfy this relation $\mathbf{T} \cdot \mathbf{S}_{\mathrm{G}}=$ $(0,0,1)$ for an arbitrary $\mathbf{T}$. The first element of the general spin rotator makes one arbitrary rotation. Then, after allowing normal spin transport to induce a $90^{\circ}$ spin phase advance and 
thereby rotating to an orthogonal axis, the second element performs the second arbitrary rotation, creating the desired $\mathrm{S}_{\mathrm{G}}$.

In 1992, the ring-to-linac (RTL) and linac solenoids were used to generate the rotations about the longitudinal axis, and the designed bend angle of the RTL extraction line served to introduce the necessary $90^{\circ}$ spin advance between theses two elements. However, as mentioned previously, the use of flat beams greatly increases the difficulty of optical matching through solenoidal field magnets. At least eight skew quadrupole magnets would be required in order to preserve emittance through such a field. Therefore a new spin rotation technique was invented: the use of the SLC arc as a spin rotator [36].

At the peak $Z$ production energy, $E_{b}=45.6 \mathrm{GeV}$, the spin tune and the vertical betatron oscillation frequency in the SLC arcs are nearly identical. This means that, although the momentum procession in a betatron oscillation cancels (the electrons move up and down a great deal, but always end up back in the same place), the spin precession does not; rather, it slowing accumulates as a net rotation about the longitudinal axis. This point is illustrated in figure 2-10.

At the beginning of the achromat, the spin is longitudinal. If the spin tune and betatron oscillation frequency were not equal, any spin precession resulting from the first half of one betatron oscillation would not add coherently with that in the second half, and on average there would be no buildup of the $y$ spin component. However, as a result of the resonance condition, there is in fact a coherent addition of spin precessions and a steady buildup of the $y$ component of spin.

In the arc rotator technique, two overlapping adjustable closed vertical orbit bumps take the place of the solenoids in the old rotation scheme. The bump settings are optimized by fitting a function to a nine-point grid search in bump space. Measurements confirm that the maximum polarization attainable at the IP by the solenoid rotator technique is duplicated by the arc rotator technique [37].

The electron bunch enters the north arc, which is composed of 23 achromats, each of which consists of twenty combined-function dipole magnets, with a polarization $P_{e}^{\text {linac }}$. Each achromat bends the momentum by $10.27^{\circ}$, which implies $1086^{\circ}$ of spin rotation about 


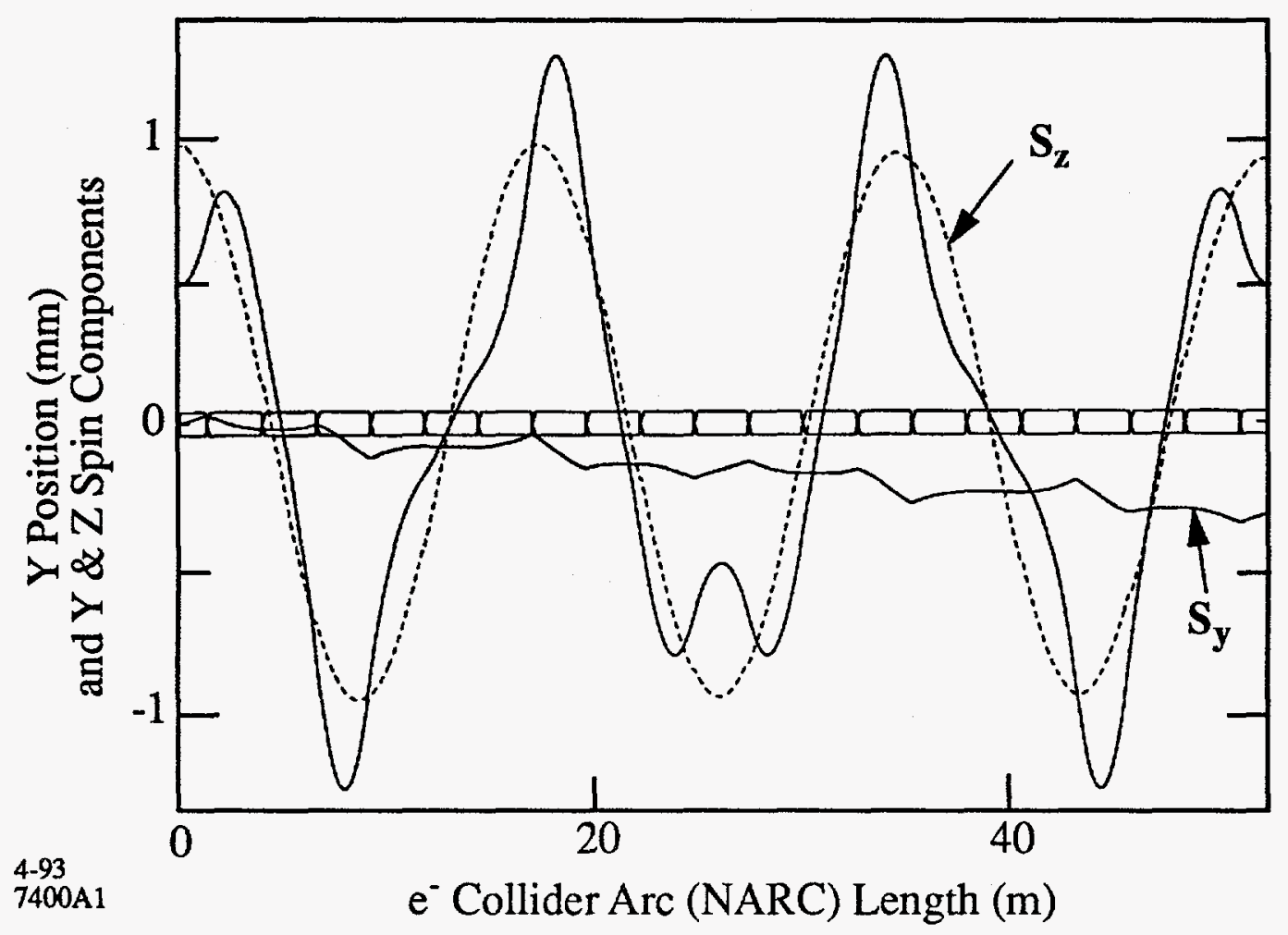

Figure 2-10: Vertical orbit and spin components over the first 23 achromat sections of the arc. The particle is launched with a vertical offset of $0.5 \mathrm{~mm}$ and longitudinal spin.

the vertical axis. Each achromat also has $1080^{\circ}$ of vertical betatron phase advance (the resonant condition). The spin is maintained vertically through the arc, until the final six achromats, wherein two large amplitude closed betatron oscillations (the spin-bump rotation system) precess the spin into the longitudinal direction. The electrons enter the final focus region with a polarization $P_{e}$, which is less than $P_{e}^{\text {linac }}$ because of the spin diffusion effect in the arcs.

\section{Spin Diffusion}

In the SLC arc, the spin tune is on the order of 100 , and therefore small differences in energy have a large effect on the spin precession. We have adopted the term spin diffusion in order to differentiate this process from "depolarization," a term which implies a com- 
plete loss of polarization information. Polarization is not irretrievably lost during spin diffusion, as we will illustrate in the following example:

Suppose the SLC electron arc consisted of perfectly uniform positive and negative vertical magnetic fields, and that the electrons are $100 \%$ longitudinally polarized. In the first section of the arc (the reverse-bend), the electron momentum is rotated exactly $\pi / 2$ radians. Following equation (2.2), the spin precesses approximately 26 times. However, the beam has some energy spread, typically $0.2 \%$ RMS, and off-energy electrons will have different precession angles. The precession angle is correlated with the energy except for the small effect of synchrotron radiation induced randomization. The precession difference $\Delta \theta$ is approximated by: $\Delta \theta=v \cdot(\Delta \mathrm{E} / \mathrm{E})=0.21$ radians. Thus the polarization of the beam at this point is about $98 \%$. After the second section of the arc, the central electrons are again rotated $\pi / 2$ radians, in the opposite direction. The polarization at this point is again $100 \%$ ! The spin diffusion has been cancelled by an equal and opposite momentum precession, and in this sense polarization is not truly lost. Diffusion occurs again through the final section of the arc, leaving $P_{e} \approx 0.98 \cdot P_{e}^{\text {linac. }}$.

Of course, the real SLC arc is not quite so simple. The arc is not perfectly planar, and the spin, which starts vertically, precesses about all three axes in a non-commuting manner. Fortunately, we directly measure the dependence of spin on energy through experiments with very low energy spread beams, where $\Delta \mathrm{E} / \mathrm{E}<0.1 \%$. Generation of such narrow energy spreads requires low current running $\left(\approx 10^{10} \mathrm{e}^{-} / \mathrm{pulse}\right)$ that is not suitable for normal physics operation. A special machine experiment was run [37], during which the central energy of the nearly monoenergetic beam was scanned, and polarization dependence on energy was measured by the Compton polarimeter. Results from this experiment are shown in figure 2-11.The data were fit to the function

$$
P_{z}^{i}=P_{2}^{C} \cdot \cos \left[2 \pi N \cdot \frac{\left(E^{i}-E_{o}\right)}{45.56}\right]
$$

where $P_{2}^{i}$ is the measured polarization and $E^{i}$ the measured energy of the $i$ th scan point, $P_{z}{ }^{C}$ was the optimized polarization measured by the Compton polarimeter at the nominal 
energy, $E_{o}$, and $N$ is the effective number of spin rotations in the arc x-z plane. The fit results give $N=17.9 \pm 0.4$, an number smaller than the 26 rotations predicted by the simple planar model, as expected, since the spin has a significant vertical component throughout the arc. This mode of operation has the advantage of lowering spin diffusion loss and also reducing the polarization-energy correlation, an important property which is discussed further in chapter 5 . The loss of polarization due to spin diffusion is approximately $1.4 \%$ for a Gaussian energy spread of $0.15 \%$.

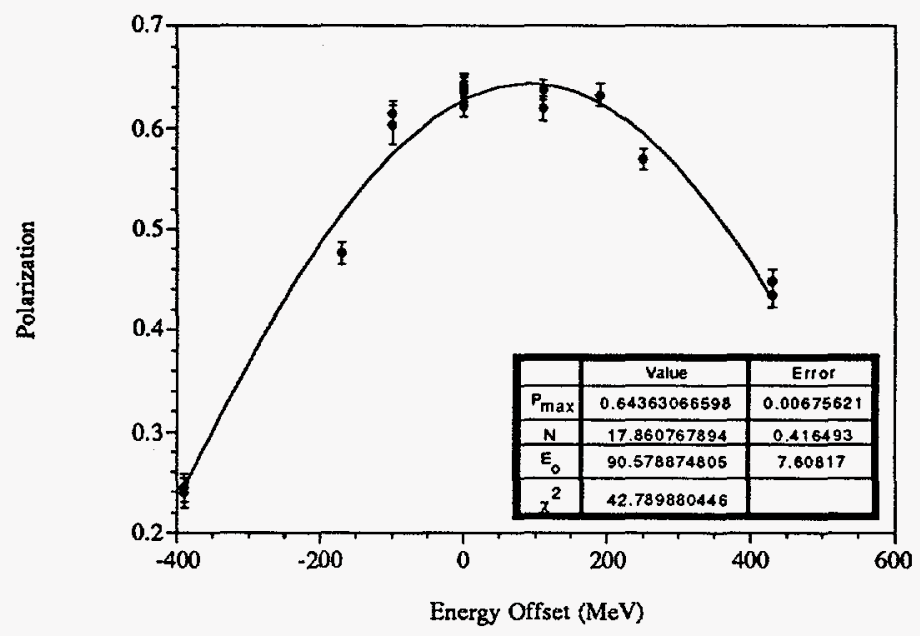

Figure 2-11: The dependence of polarization on energy

\subsection{Møller Polarimetry}

In the $A_{L R}$ experiment, it is of course necessary to monitor the polarization of the electron beam. There are two methods employed to do this, both taking advantage of spin-dependent QED cross-sections. One method is Møller (electron-electron) scattering, and the other is Compton (electron-photon) scattering. Each system requires a polarized target and a detector for the scattering products. The combination of target, detector, and data acquisition system is referred to as a Polarimeter.

Møller polarimetry is based on the scattering of electrons off of polarized atomic electrons in a magnetized target [38]. In the case where the center-of-mass energy of the two 
electron system, $s$, is much greater than the electron mass squared, the tree-level cross section for the scattering process in the center-of-mass frame is given by

$$
\frac{d \sigma}{d \Omega}=\frac{\alpha^{2}}{s} \cdot \frac{\left(3+\cos ^{2} \theta\right)^{2}}{\sin ^{4} \theta}\left[1-P_{B}^{z} P_{T}^{z} A^{z}(\theta)-P_{B}^{t} P_{T}^{t} A^{t}(\theta) \cos \left(2 \varphi-\phi_{B}-\phi_{T}\right)\right]
$$

where $\alpha$ is the fine structure constant, $\theta$ is the center-of-mass frame scattering angle, and $\varphi$ is the azimuthal angle of the scattered electrons. The subscripts $B$ and $T$ refer to the beam and target, respectively; $P^{2}$ is the longitudinal polarization, $P^{t}$ is the transverse polarization, and $\phi$ is the azimuth of the transverse polarization vector. Finally, $A^{z}(\theta)$ and $A^{t}(\theta)$ are the longitudinal and transverse asymmetry functions, given by

$$
\begin{aligned}
& A^{z}(\theta)=\frac{\left(7+\cos ^{2} \theta\right) \sin ^{2} \theta}{\left(3+\cos ^{2} \theta\right)^{2}} \\
& A^{t}(\theta)=\frac{\sin ^{4} \theta}{\left(3+\cos ^{2} \theta\right)^{2}}
\end{aligned}
$$

These asymmetry functions are maximized at a scattering angle of $90^{\circ}$ in the center-ofmass frame, and approach zero in the forward and backward scattering limits.

In a general Møller polarimeter, the electron beam strikes a magnetically polarized target. A collimator downstream of the target defines the angular range (and thereby the momentum range) and azimuth of the scattered electrons that continue towards the detector. A dipole magnetic field decouples the momentum and scattering angle, and further separates the scattered electrons from the main beam, reducing detector backgrounds. The Møller electrons then appear in the detector as an elastic scattering peak. The signal asymmetry in a given detector channel, $A_{N}$, is formed by the observed number of Møller electrons, $N_{M}$, for positive and negative beam/target polarization products such that

$$
\begin{aligned}
A_{N} & =\frac{N_{M}\left(+P_{T} P_{B}\right)-N_{M}\left(-P_{T} P_{B}\right)}{N_{M}\left(+P_{T} P_{B}\right)+N_{M}\left(-P_{T} P_{B}\right)} \\
& =-P_{B}^{z} P_{T}^{z} a^{z}-P_{B}^{t} P_{T}^{t} a^{t} \cos \left(2 \varphi-\phi_{B}-\phi_{T}\right)
\end{aligned}
$$


where the longitudinal and transverse analyzing powers, $a^{2}$ and $a^{t}$, are complex convolutions of the detector acceptance, the momentum distribution of the target electrons, and the asymmetry functions defined in (2.6).

There were originally two Møller polarimeters designed for the SLC, the Linac Møller and the Extraction Line Møller. Each of these designs employs the same target material and target assembly.

\subsubsection{Møller Targets}

Both polarimeters use permendur ${ }^{1}$ foils of various thicknesses and orientations as targets. The magnetization density of a foil is measured in the laboratory, and the target polarization, corrected for spin-orbit effects, is extracted from this measurement. A typical target is around $8 \%$ polarized when placed in a saturating magnetic field. The foil polarization measurement is the limiting systematic uncertainty in Møller polarimetry, introducing a relative error of approximately $3 \%$.

Within either polarimeter, five target foils are mounted on an aluminum target holder whose position relative to the electron beam can be changed remotely. A set of three Helmholz coils generate a saturating field of 120 Gauss in any direction around the target. All analyzed data makes use of longitudinal foils, canted $20^{\circ}$ with respect to the beam, saturated by longitudinal magnetic fields. By switching the sign of the magnetic field between runs, systematic effects related to background and current asymmetries are minimized.

\subsubsection{The Extraction Line Møller Polarimeter}

The original Extraction Line Møller (XLM) polarimeter design (figure 2-12 A) was found to be incompatible with the system of energy spectrometer magnets. Electrons that lose energy through bremsstrahlung in the Møller target are dispersed by the vertical bend magnet of the spectrometer and strike apertures, flooding the downstream area (including the detector) with background radiation. Various shielding arrangements proved ineffective, and the project was shelved in 1991.

1. Permendur (actually Va2-permendur) is an alloy, consisting of $49 \%$ Iron, $49 \%$ Nickel, and $2 \%$ Vanadium, with high magnetic susceptibility. 
During the summer of 1993, an apparently unresolvable difference between the Compton and Linac Møller polarimeter measurements prompted a revived interest in the XLM. A new design, shown in figure 2-12 B, was proposed, wherein the energy spectrometer magnets are turned off, the WISRD itself is removed, and the Møller scattered electrons are separated from the main beam by a septum magnet.

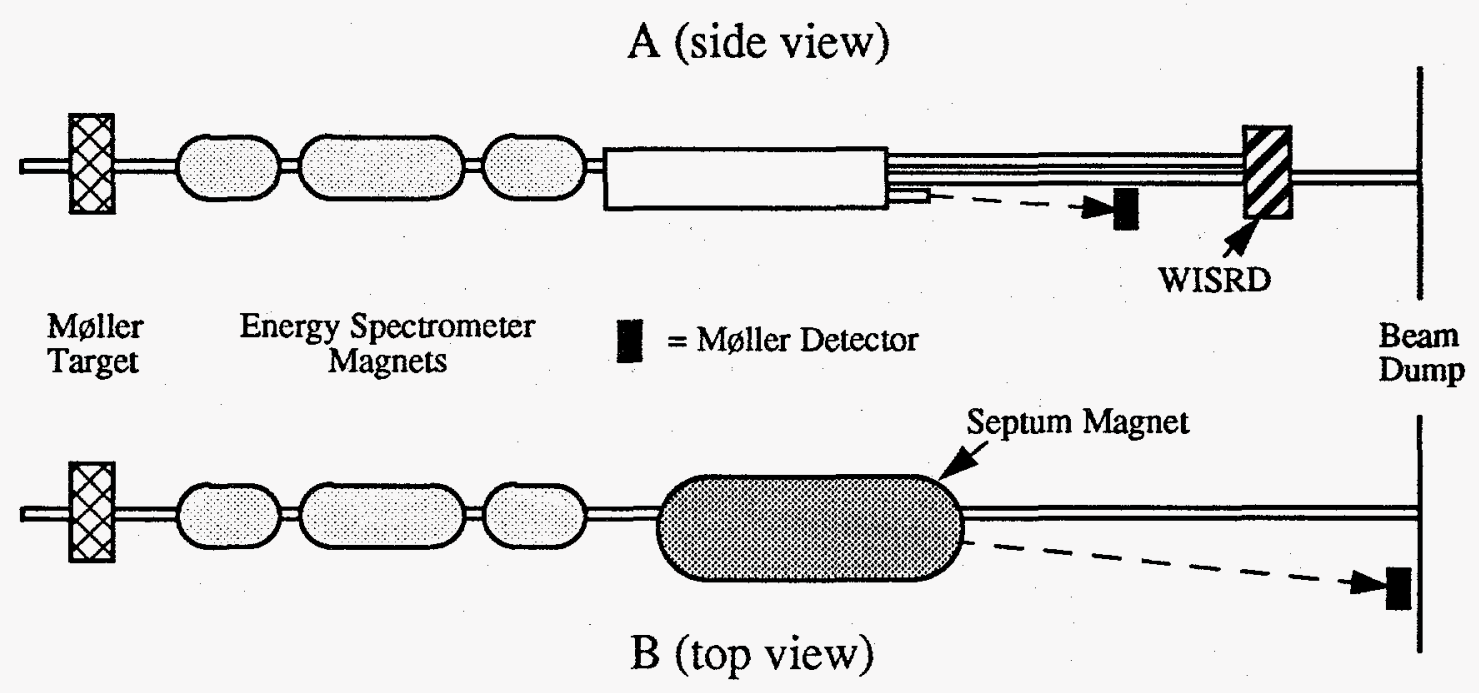

Figure 2-12: The extraction line Møller polarimeter (A) The original layout. (B) The 1993 layout.

A massive concerted effort commissioned the XLM in just over three weeks. Unfortunately, aperture problems limited the usefulness of the data taken with the new system. However, during the course of XLM data analysis, an essential aspect of Møller polarimetry was realized. The atomic binding energy of the target electrons has a large (10-15\%) effect on the scattering peak which is apparent in detectors with high angular resolution [29]. This so-called "Levchuk Effect," turns out to be the key to the reconciliation of the polarization measurements at SLAC. This effect was applied to the analysis of the Linac Møller Polarimeter, described in the next section. 


\subsubsection{The Linac Møller Polarimeter}

The Linac Møller Polarimeter (LMP) is located at the end of the linac in the old PEP extraction line, and is used to make diagnostic measurements before the electron beam enters the north arc. It is an intrusive measurement, as the 40B1 septum magnet must be turned on in order for electrons to reach the LMP target, and the linac solenoid must be turned on in order to measure longitudinal polarization.

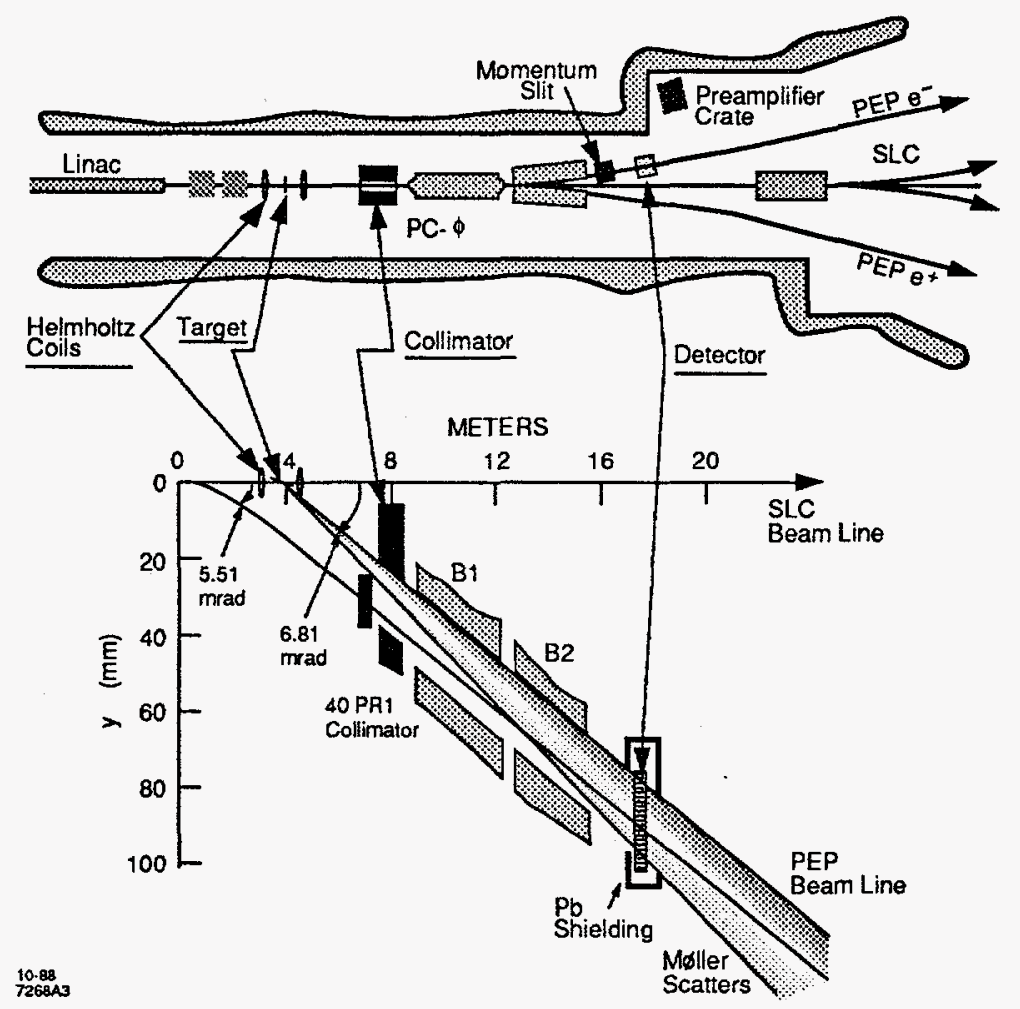

Figure 2-13: The linac Møller polarimeter.

The collimator shown in figure 2-13 (labelled PC-0) selects the azimuth of scattered electrons. The scattering plane of the collimator is chosen to be perpendicular to the bending plane of the magnet in order to decouple the scattering angle from the momentum. A horizontal aperture located $3.54 \mathrm{~m}$ downstream of the effective bend center selects electrons in the momentum range $14.5 \pm 0.5 \mathrm{GeV}$. This corresponds to a central center-of-mass 
scattering angle of $112^{\circ}$. This operating point is slightly away from the peak asymmetry angle of $90^{\circ}$, but is less sensitive to background contamination from radiative nuclear scattering (the Bethe-Heitler process). The scattered electrons then appear on a 64 channel position-sensitive detector as a kinematic scattering peak on top of a smooth background. The detector itself consists of a two radiation length tungsten/lead radiator in front of a silicon strip detector. The scattering angle resolution of the detector is about 27 microradians.

Dedicated running with the LMP took place on eight different occasions during the 1993 run. An analysis of these runs [38], taking into account the Levchuk effect, yields the results listed in table 2-1.

\begin{tabular}{|c|c|c|c|}
\hline Experiment & $\begin{array}{c}\text { Beam } \\
\text { Energy } \\
(\mathrm{GeV})\end{array}$ & $\begin{array}{c}\text { Target } \\
\text { Thickness } \\
\text { (microns) }\end{array}$ & $\begin{array}{c}\text { Longitudinal } \\
\text { Polarization } \\
(\%)\end{array}$ \\
\hline \hline 1 & 46.6 & 49 & $62.9 \pm 1.8$ \\
\hline 2 & 46.6 & 49 & $67.9 \pm 1.9$ \\
\hline 3 & 40.6 & 49 & $65.4 \pm 2.6$ \\
\hline 4 & 46.6 & 156 & $69.2 \pm 2.4$ \\
\hline 5 & 46.6 & 49 & $67.3 \pm 2.3$ \\
\hline 6 & 46.6 & 49 & $65.9 \pm 2.1$ \\
\hline 7 & 46.6 & 49 & $63.4 \pm 2.3$ \\
\hline 8 & 46.6 & 49 & $65.6 \pm 2.5$ \\
\hline
\end{tabular}

Table 2-1: Preliminary results of Linac Møller Polarimeter measurements.

The average of all LMP measurements is $(65.8 \pm 2.7) \%$ where the error is primarily systematic. This result is in agreement with the special Compton experiments with narrow energy spread that also measure $P_{e}^{\text {linac }}$.

\subsection{The Compton Polarimeter}

The primary system for monitoring beam polarization is the Compton Polarimeter, which consists of a polarized photon target in the form of a circularly polarized laser beam, an ana- 
lyzing bend magnet, two Compton-scattered electron detectors, and a data acquisition system. A brief overview of the polarimeter will be presented, followed by detailed descriptions of the polarimeter components.

The Compton Polarimeter is represented schematically in figure 2-14. Electrons travel 33 meters from the SLD interaction point and reach the Compton interaction point (CIP) before encountering any dipole fields and therefore before any spin precession occurs. Therefore the longitudinal component of spin measured by the Compton should be the same at both points (refer to section 5.3.3 for further discussion).

The Compton polarized target consists of monoenergetic, circularly polarized photons created in a frequency-doubled Nd:YAG laser. The laser is housed in a building on the south side of the main collider hall (the "laser shack"). The beam is steered down a vent shaft and in to the SLC vacuum by a series of mirrors, and focussed at the Compton interaction point by a lens.

At the CIP, the beam electrons $\left(E_{b}=45.64 \mathrm{GeV}\right)$ collide with the target photons $\left(E_{\gamma}=\right.$ $2.33 \mathrm{eV}$ ), a collision comparable to a freight train colliding with a fruit-fly. The maximum laboratory scattering angle of Compton electrons is $9.1 \mu \mathrm{rad}$, which is smaller than the beam divergence. As a result, the scattering products do not separate from the main beam until they pass through the analyzing bend magnet, which actually consists of two magnets (SB1 and B1) whose effective bend center is approximately $3.6 \mathrm{~m}$ upstream of the polarimeter detectors.

The spin-dependent cross-section for the electron-photon scattering process has the following form

$$
\left(\frac{d \sigma}{d E}\right)_{\text {Compton }}=\left(\frac{d \sigma}{d E}\right)_{\text {unpolarized }}\left[1-P_{\gamma} P_{e}^{z} A_{e \gamma}^{z}(E)\right]
$$

where $E$ is the scattered electron energy, $P_{\gamma}$ and $P_{e}$ are the photon and electron polarizations, and $A_{e \gamma}^{z}(E)$ is the longitudinal asymmetry function. The complete details of Compton scattering are discussed in chapter 3 . The analyzing bend magnet converts the energy dependence of this cross section into a spatial dependence across the faces of the Compton 


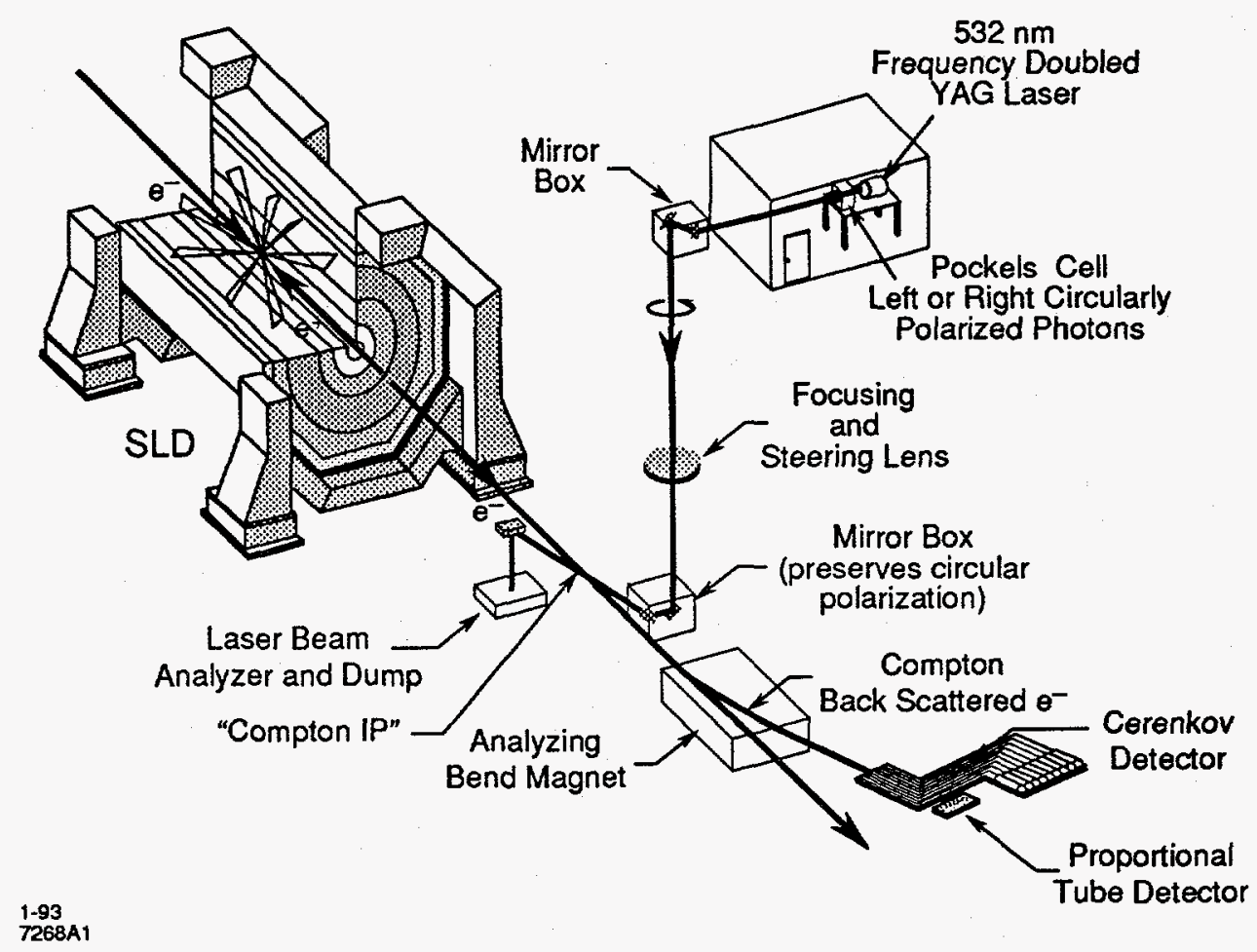

Figure 2-14: The Compton polarimeter

detectors. The detectors observe an energy dependent asymmetry spectrum, and the measurement of the observed counting asymmetry in a given detector channel allows the determination of $P_{e}$, given the target polarization $P_{\gamma}$

The total cross-section for this process is large (hundreds of millibarns). We estimate on the order of one thousand Compton electrons are produced each beam crossing during which the target laser is fired.

\subsubsection{The Compton Polarized Target}

The polarized target of the Compton Polarimeter consists of $2.33 \mathrm{eV}$ photons. These photons are created in the resonant cavity of a Spectra Physics GCR-11 frequency-doubled Nd:YAG (Neodymium:Yttrium-Aluminum-Garnet) laser. The solid YAG rod is pumped into an excited state by flash lamps, and resulting atomic transitions produce $1064 \mathrm{~nm}$ photons. The photons leave the laser cavity in short $(8 \mathrm{~ns})$ high-power $(150 \mathrm{~mJ})$ bursts at a rate 
of $10.91 \mathrm{~Hz}$ (every 11 th SLC beam crossing). This rate was chosen in order to sample all 120 accelerator time slots and to avoid any harmonic of the line frequency, and thus to avoid any timing-related biases. This means that the Compton background is sampled ten times for each signal point. After leaving the cavity, the beam passes through a frequencydoubling crystal, which uses a nonlinear optics phenomenon to combine two $1024 \mathrm{~nm}$ photon states into a single $532 \mathrm{~nm}$ state. The frequency-doubled beam passes through and infrared separator to assure that there is no low-energy photon contamination.

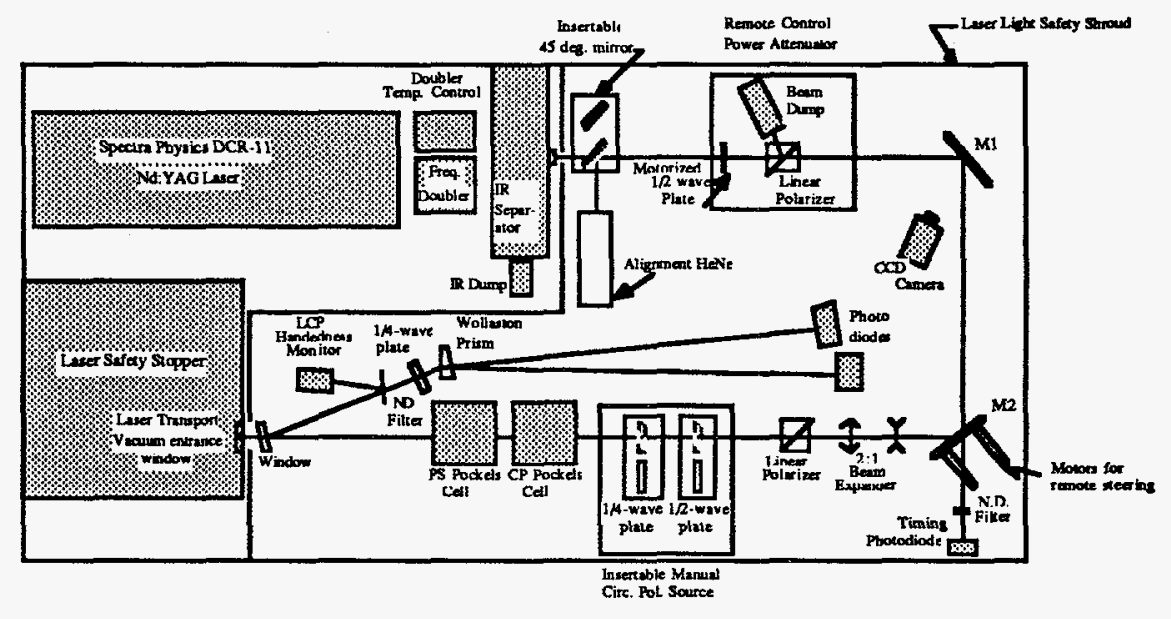

Figure 2-15: The CLS laser bench.

Referring now to figure 2-15, the frequency-doubled beam first passes through a remotely adjustable half-wave plate which acts as a power attenuator. The beam is then reflected off of two mirrors and, even though the beam exits the laser head already highly polarized, it is nevertheless passed through an additional optical element in order to assure $100 \%$ linear polarization. The beam is then expanded by a Galilean-type lens configuration, in order to minimize energy density (and therefore damage) on optical components of the laser transport system. The linearly polarized light then passes through two Pockels cells.

The first cell is referred to as the Circular Polarization Pockels Cell (CP PC), because when the linear polarized light passes through it, it becomes almost completely circularly 
polarized. The principle axes of this cell are tilted by $45^{\circ}$ with respect to the linear polarization of the incident light. The second Pockels cell is referred to as the Phase Shift Pockels cell (PS PC) because its primary effect is to compensate for phase shifts introduced by the beam optics and transport system between the laser bench and the Compton interaction point. The principle axes of this cell are either parallel or perpendicular to the axes defined by the initial linear light. Thus the retarding axes of the PS Pockels cell are aligned $45^{\circ}$ with respect to the axes of the CP Pockels cell.

Because the optical elements of the transport line introduce arbitrary phase shifts and phase rotations, the Pockels cell voltages were scanned continually throughout the run. There were two types of scans: the photodiode scan and the Compton asymmetry scan. Photodiode scans were made once per hour and took approximately 1.2 minutes each. The scans look at the degree of extinction it is possible to create in the CP analysis photodiodes. This allows us to characterize the amount of unpolarized light in the photon beam, which was found to be typically $0.5 \%$. The Compton asymmetry scans on the other hand compared the Compton asymmetry measure for various Pockels cell high voltage settings. In other words, the photodiode scans told us the maximum achievable circular polarization, while the electron polarization scans told us how far the nominal PC voltages were from obtaining this maximum polarization. This number was found to be typically 50-80 volts, corresponding to nominal photon polarization of approximately $99 \%$ (0.5\% lost to unpolarized light and $0.5 \%$ lost to uncompensated phase shifts in the transport optics). The analysis of the laser circular polarization will be discussed completely in chapter 3 .

Returning to the discussion of the laser transport (now referring to figure 2-16), the expanded beam enters the transport system through a vacuum window (labelled "LTL ENT W"). In the full transport system, the laser beam passes through a total of four vacuum windows, all of which were measured to have negligible birefringence at vacuum and atmospheric pressures. A reflection from a pick-off plate is directed into the laser bench 


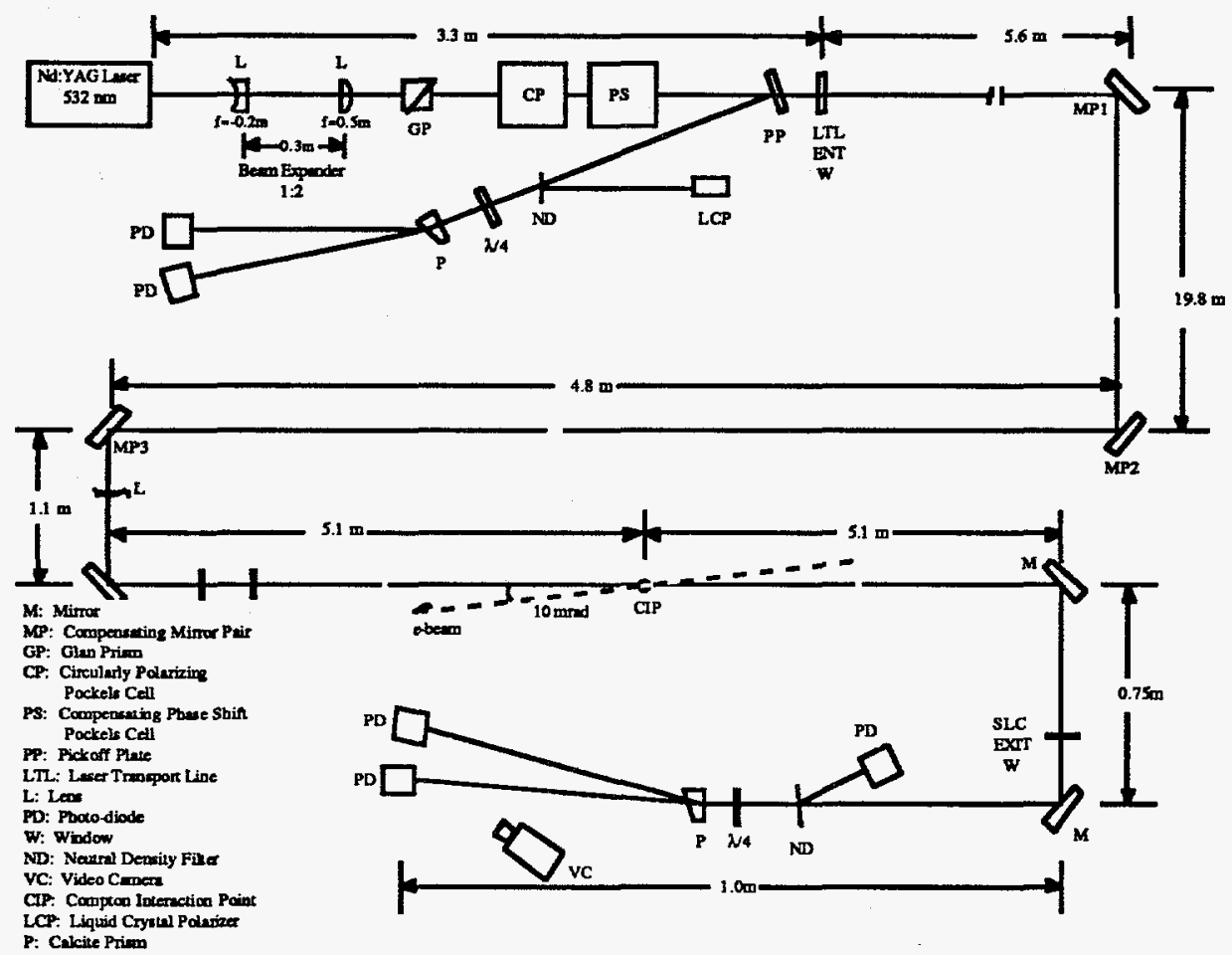

Figure 2-16: The Compton laser transport system.

$\mathrm{CP}$ analysis optics. These photodiodes monitor the circular polarization of the light, $P_{\gamma}$ as it enters the transport system through the following relation

$$
P_{\gamma}=\frac{2 \sqrt{R}}{1+R}
$$

where $R$ is the ratio of the signals in the two photodiodes.

In addition, a reflection into the Liquid Crystal Polarizer (LCP) photodiode provides a monitor of the handedness of the circular polarization. Recall at this point that the particle physics convention for handedness is opposite to the optics convention. The optics convention is based on the direction of rotation of the electric field vector of the light wave front. Particle physicists naturally prefer to consider a right-handed photon to have its angular momentum pointing in the direction of its travel (and thus follows the "right-hand-rule"). 
In this convention, right-handed photons and right-handed electrons have the same momentum characteristic. The particle physics convention is adhered to throughout this document. Note that there are two reflections which ensures that the handedness going into the LCP is the same as that entering the transport line.

The transport system itself was at first evacuated, then later run with nitrogen, and finally filled with 3-5 psi overpressure of helium, in order to minimize optics damage. 1993 running was plagued by optics damage; much more so than 1992. For example, the YAG rod itself and the Q-switch optics were both replace once during the 1993 run; neither had any problems during the 1992 run. This was probably due to degradation of the resonant cavity, which caused "hot-spots" (points of very high energy density) to appear in the laser beam profile. Such hot spots quickly react with any impurities and escalate damage and burns on optical surfaces.

An important component of the laser transport system is the mirror-box. The mirrorbox is a construction that allows the beam to be reflected $90^{\circ}$ while maintaining the photon helicity. This is accomplished by having the beam bounce off of two mirrors instead of one for any change of direction, so that the polarization changes sign after the first bounce, and then changes sign again after the second bounce. Also, by using identical (i.e. manufactured at the same time) dielectric mirrors, any phase-delay introduced by surface effects in the first mirror is exactly compensated for and removed by the second mirror.

After the CLS laser beam passes through three such mirror-boxes, it enters the lens box, where a remotely moveable $5 \mathrm{~m}$ focal length lens focuses and steers the beam for collision with the electrons. After one more mirror-box reflection, the photons enter the SLC vacuum through a window at an angle of 10 milliradians with respect to the electron beam. At the CIP, the RMS electron beam size is 350 microns, and the photon beam size is 500 microns. After collision, the laser beam exits the SLC vacuum, and is reflected by an uncompensated mirror into the laser analysis box. The analysis box contains $\mathrm{CP}$ analysis optics in the same configuration as on the laser bench, see figure 2-17. The final uncompensated reflection introduces a large phase shift between the CIP and the analysis box, which is why the analysis box data cannot be taken as a direct measurement of the laser polarization at the CIP. 


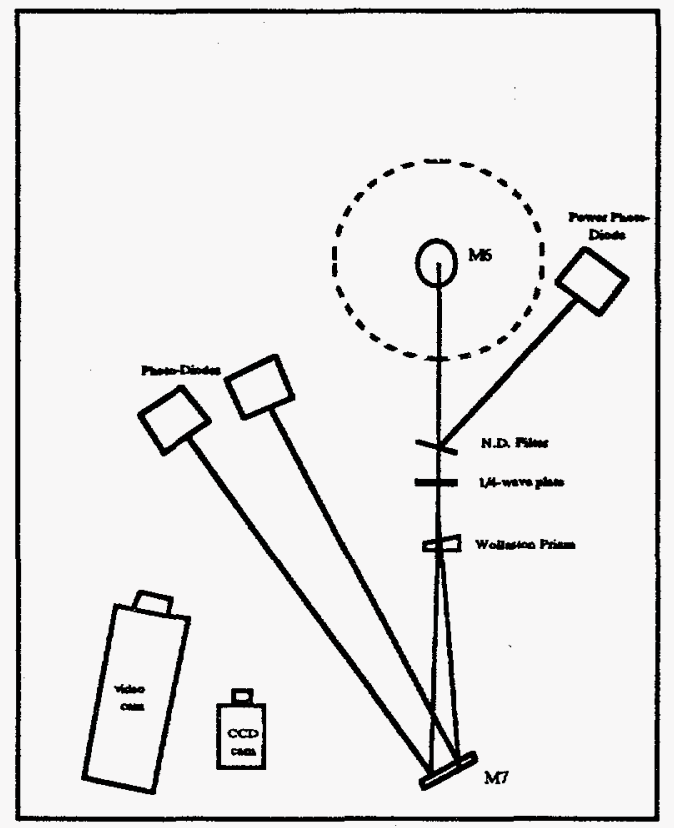

Figure 2-17: The CLS analysis box optics

\subsubsection{The Cherenkov Detector}

The ideal polarimeter detector requires good position resolution and background suppression. The position spectrum of the scattered electrons must be carefully measured in order to extract the beam polarization. With these requirements in mind, a simple Cherenkov design was selected. The Cherenkov detector consists of nine $1 \mathrm{~cm} \times 1 \mathrm{~cm}$ polished aluminum channels, filled with static sys-trans-2-butene gas at atmospheric pressure, ending at nine Hamamatsu R1398 photomultiplier tubes with custom bases designed at the Lawrence Berkeley Laboratory.

The phototube base is also an important component of the overall detector design. In order to fully calibrate the detector, it is necessary to determine the linearity characteristics of the phototubes. This is done by monitoring the asymmetry in one channel with a fixed signal height, and comparing this to the asymmetry measure in a channel whose signal height is varied. As the phototube saturates, the larger (parallel electron-photon spins) signal is measured lower than its true value, and the asymmetry in this channel decreases relative to the control channel. The problem is, that in order to change the signal height in 


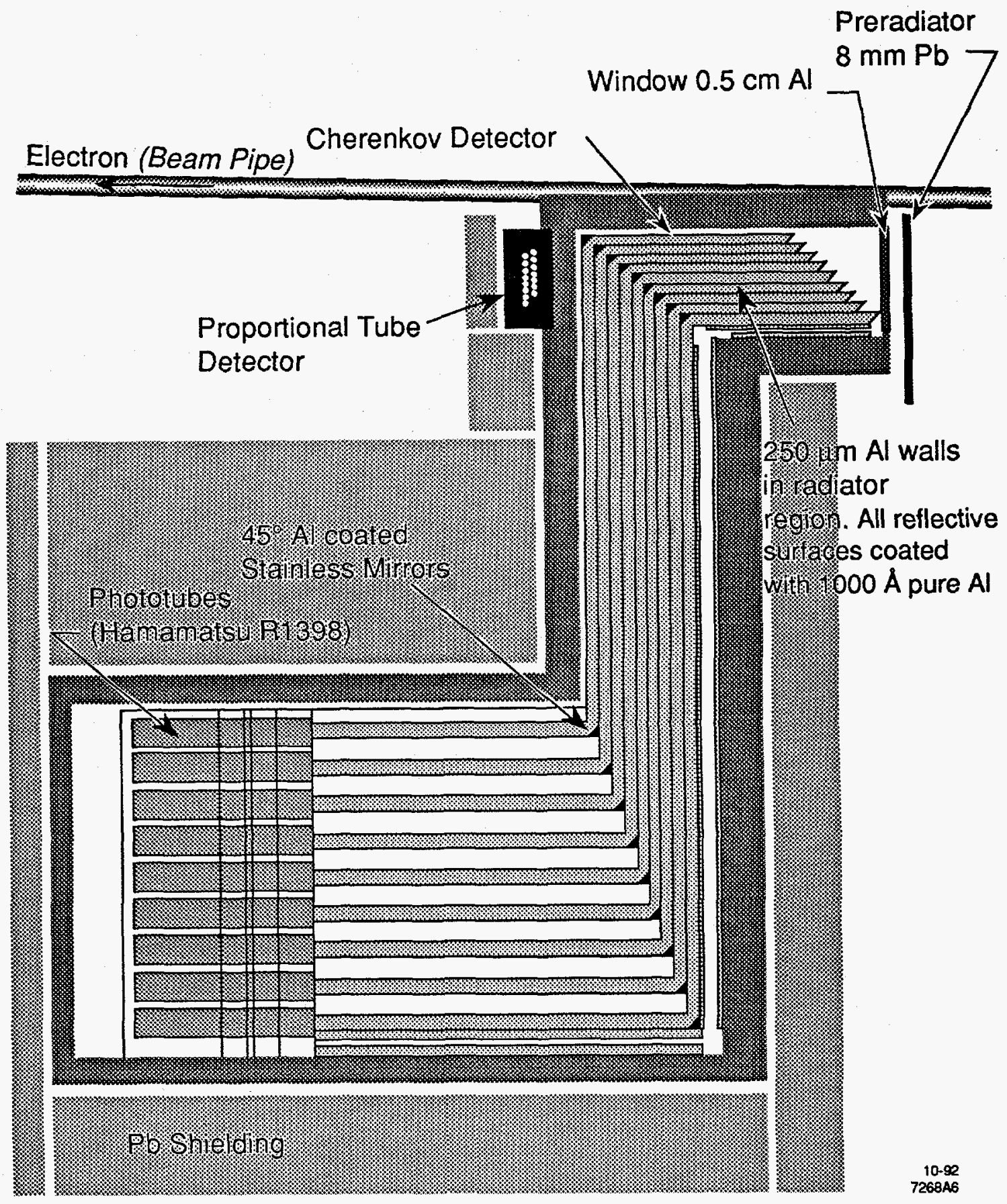

Figure 2-18: Overhead view of the Compton Cherenkov and proportional tube detectors. 
a channel, one must change the gain of the phototube, which in turns changes the spacecharge characteristics of the amplifying stages, which in turn changes the saturation characteristic. The purpose of the LBL phototube base design is to allow the voltage on the front-end of the dynode chain to be varied, thus changing the gain of the phototube, and yet keep the back-end voltages fixed, thus maintaining a constant space-charge and thereby a constant saturation characteristic. Without this important characteristic, any measurement of the phototube linearity involving gain adjustments would be suspect. This base design is discussed in greater detail in Reference [39].

When Compton-scattered electrons leave the SLC vacuum through a thin window, they pass through a lead radiator. The high energy shower products enter the Cherenkov channels through a $0.5 \mathrm{~cm}$ aluminum window, where they travel faster than the speed of light in that medium and thus emit Cherenkov light (the Cherenkov threshold of the gas is approximately $10 \mathrm{MeV}$ ). This light travels down the aluminum channels, is reflected off of polished, aluminum-coated stainless steel mirrors, and is detected in the photomultiplier tubes. The amount of Cherenkov light produced is proportional to the number of Compton scattered electrons.

The Cherenkov detector housing is surrounded by lead in order to shield the phototubes from any backgrounds. The detector assembly rests on a thick aluminum plate, which moves perpendicular to the beam-line on a set of precision bearings. The plate (henceforth referred to as the table) is driven by a stepping motor and its position is monitored by a linear potentiometer. The table is nominally at the inner-most position (as close to the main beam as possible), resting against a physical stop. The potentiometer reference voltage and position voltage are read by a SAM (smart analog module) at the end of every polarimeter run, so that the detector has no systematic uncertainty associated with the table being out of position.

The table movement is a useful tool for the determination of the absolute location of the detector relative to the main beam, which is in turn an essential input for the calibration of the detector analyzing powers. Such a determination is called an edge scan. Edge scans rely on the fact that the Compton cross-section has a sharp kinematic endpoint, corresponding 
to the full recoil of scattered electrons in the center-of-mass frame and the maximum amount of energy the electrons can lose. As the detector table is moved, the signal in the outer channels systematically drops. By comparing this data to Monte Carlo, the absolute position of the detector is precisely calibrated (see section 3.4.1 for further details and the estimate of systematic errors).

Another important piece of detector hardware is the lead pre-radiator assembly. This assembly allows the remote insertion or removal of two lead plates of different thicknesses $(1 / 3$ " and $2 / 3$ ") for a total of four different pre-radiator thicknesses. The purpose of the preradiator is to enhance the signal-to-background ratio. An unfortunate side-effect is that the Compton spectrum is smeared across channel boundaries, which does affect the analyzing power of each channel. The affect of these different radiator thicknesses is modelled in a Monte Carlo simulation, but the correction to the analyzing powers is only on the order of $1 \%$ in the outer channels (again, see the calibration section for a more complete discussion).

\subsubsection{The Proportional Tube Detector}

When the extraction line Møller project was shelved in 1991, the detector, electronics, and data acquisition for this system were already in place. It was decided to try to adapt these components as a back-up detector for the Compton polarimeter. The original XLM proportional tube detector proved to be very sensitive to backgrounds in the Compton area, so a new detector, optimized for that location, was constructed.
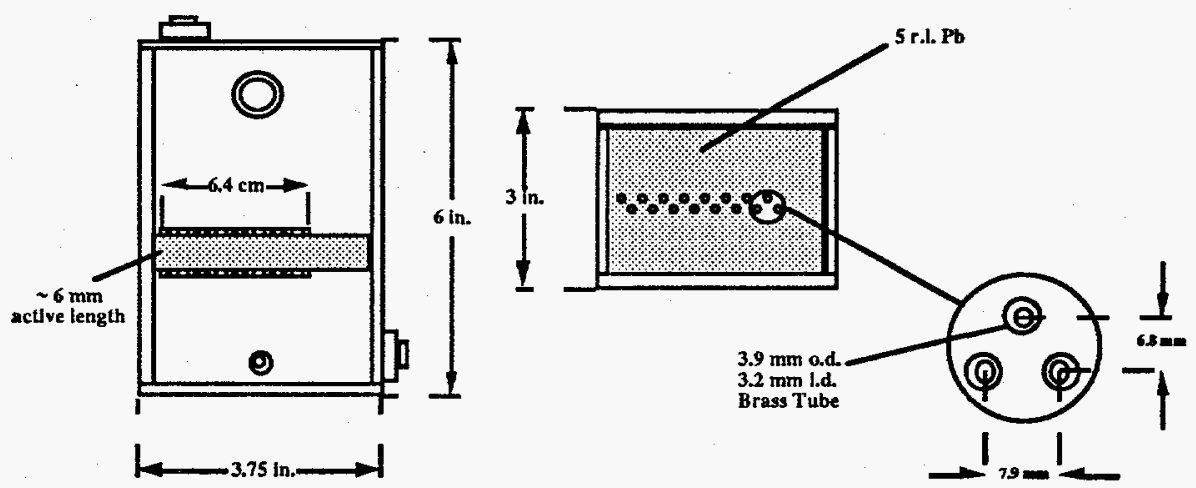

Figure 2-19: The Compton proportional tube detector. 
The Compton proportional tube detector (PTD) consists of sixteen brass tubes imbedded in a lead brick which provides 5 radiation lengths of radiator for Compton scattered electrons that have already passed through the Cherenkov detector. In the middle of each tube is a 20 micron diameter wire which is charged to -750 volts. Particles ionize in the detector gas (so called $H R S$ gas, composed of $89 \% \mathrm{Ar}, 10 \% \mathrm{CO}_{2}$, and $1 \% \mathrm{CH}_{4}$ as a quencher), and the ionized particles cause a charge avalanche to occur in the very high electric fields near the sense wire.

The induced signal is capacitively coupled to the same prototype amplifiers that were designed for the calorimeter tophats (see section 2.5.2). These amplifiers shape the signal into a long $(2 \mathrm{~ms})$ pulse which is transmitted over twisted pair cables with signal pulse transformers on either end for noise reduction. The final signal is read by a LeCroy 2259B peak-sensing ADC. Unfortunately, as it was later discovered, the linearity characteristics of the $2259 \mathrm{~B}$ are particularly bad in the low signal range.

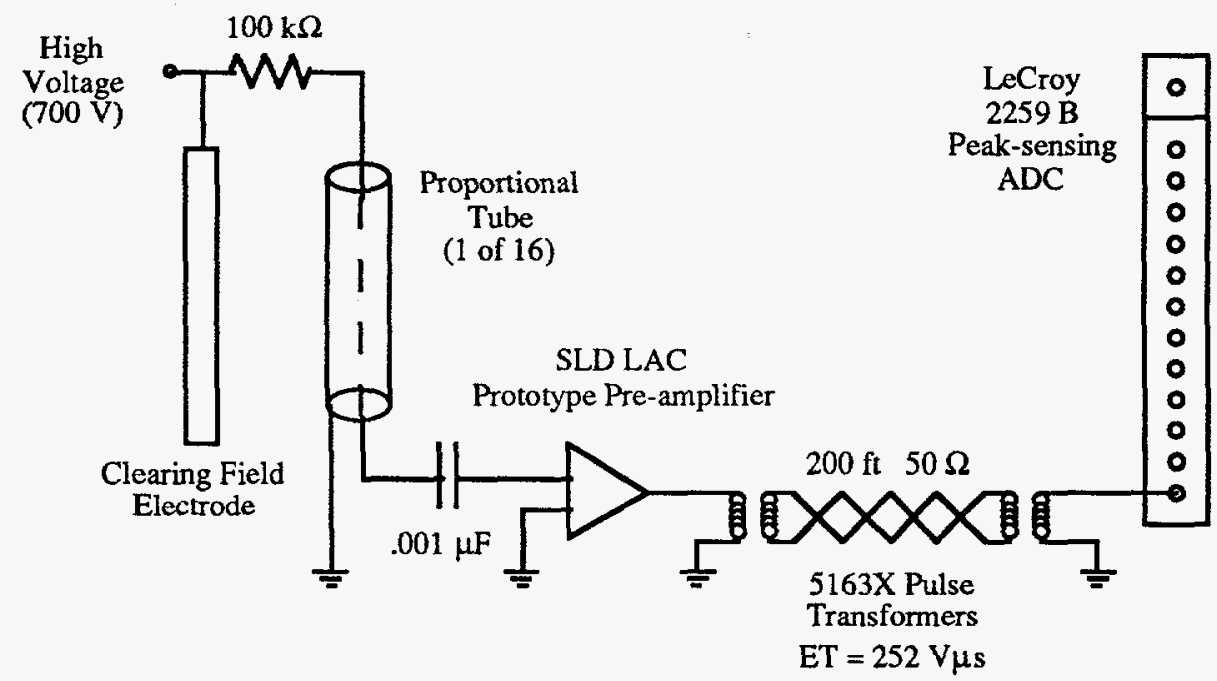

Figure 2-20: The PTD electronics

These linearity problems are demonstrated in figure 2-21, which shows the asymmetry measured in an PTD channel, normalized to Cherenkov channel 6 (whose linearity is well- 
understood, see section 3.3.2) as a function of the PTD pulse height. So far, the PTD system has not provided a precise independent measurement of the beam polarization, as hoped.

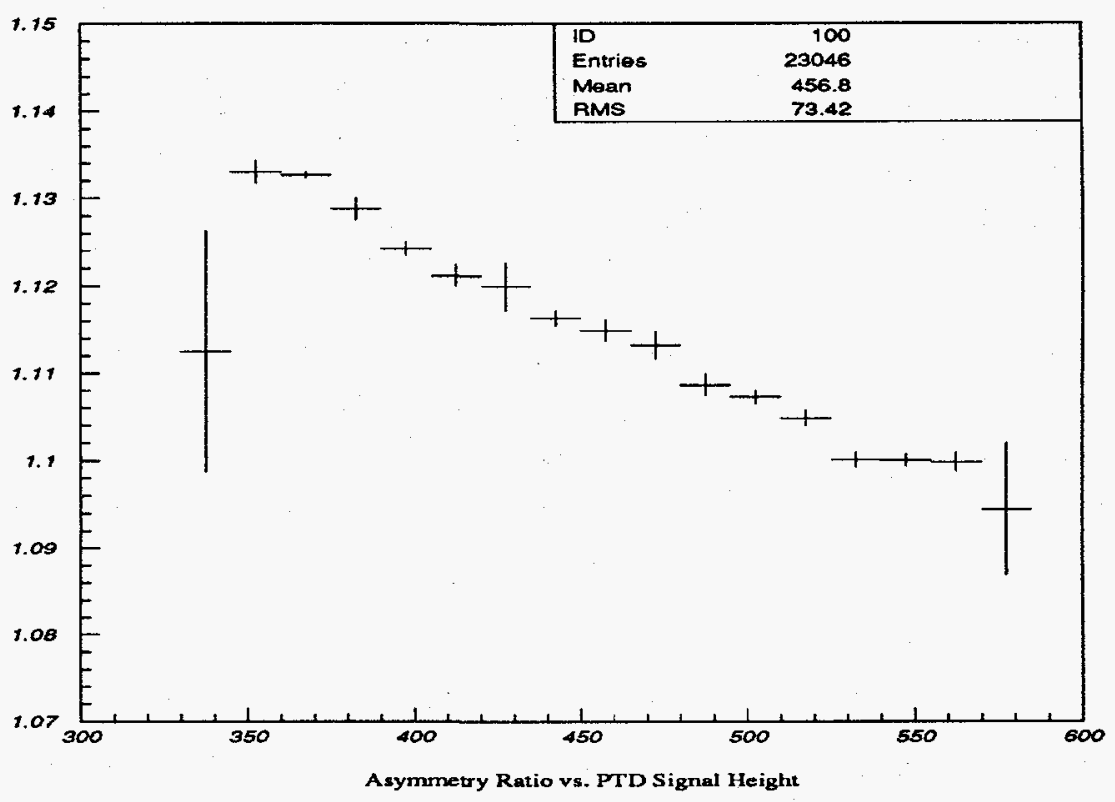

Figure 2-21: The PTD linearity from 1993 data.

\subsubsection{Data Acquisition}

The polarimeter data acquisition, represented in figure 2-22, consists of three stages: The CAMAC crates, the M2ELN microVax, and the SLDACQ VAX. One CAMAC crate is located in the mezzanine above the SLD control room; two others are located in the laser shack. The microVax is also located in the SLD mezzanine, directly above the first CAMAC crate. The interface between the microVax and the CAMAC crates is a Kinetic Systems Serial Highway driver. Ribbon cables connect M2ELN to the first CAMAC crate. A fiber optic line connects crate one to the laser shack crates 2 and 3. M2ELN is in turn linked to the SLDACQ VAX cluster via an ethernet connection. 


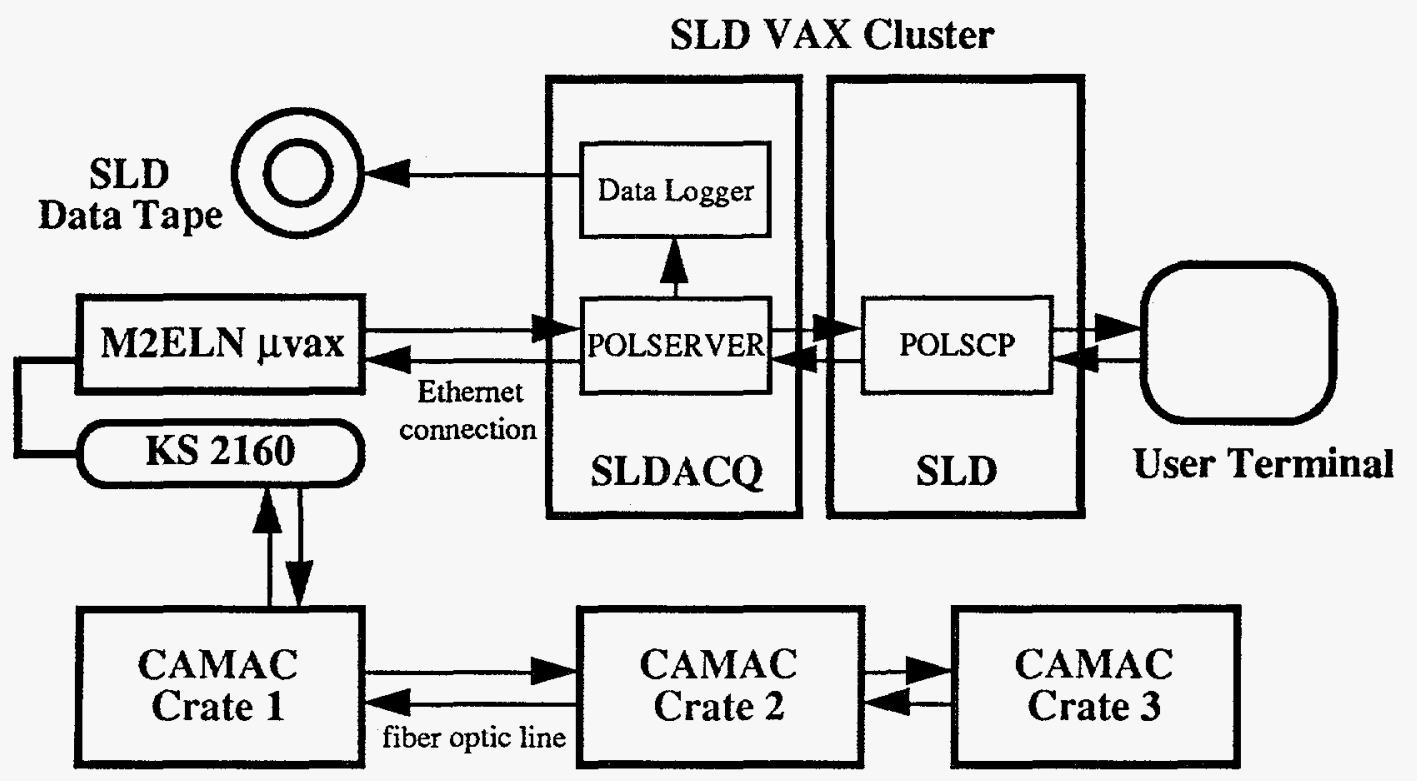

Figure 2-22: Schematic representation of the Compton polarimeter data acquisition

Every beam crossing, an SLC base rate trigger (TRBR) triggers ADC gates on modules in the three CAMAC crates. These triggers are farmed out through delay generators in order to properly gate each module. The very last gate to be initiated triggers a CAMAC LAM ("Look-At-Me") which causes the M2ELN microVax to read all of the modules. Fifty-three words (106 bytes) of Polarimeter data are collected every beam crossing. This raw data is stored in a local ring buffer. Once 150 beam crossings have been accumulated in the ring buffer, M2ELN sends the entire buffer to the SLD VAX via ethernet, where it is handled by the POLSERVER process.

POLSERVER logs data in two modes simultaneously. In the first mode, the raw data packet of 150 beam crossings is written to SLD data tape in a sparsified manner, with only one background (laser off) state per signal (laser on) state, instead of the available ten to one ratio, in order to minimize tape input/output. Background pulses are chosen two beam crossings before the signal crossing, in order to measure background on the same (even or odd) time slot, with no signal-related bias. During 1993, raw polarimeter data accounted for ten to twenty percent of data written to SLD tape. 
In the second mode, histograms of sums and sums-squared are accumulated by the POLSERVER process. The PLS helicity state is decoded from the crate 1 register and can have three possible values: left-handed, right-handed, and error. During the 1993 run, the Polarimeter relied on the Mach line transmission of the PLS helicity bits. The CLS helicity state is decoded from the crate 3 data register and can have four possible values: lefthanded, right-handed, unpolarized, and off. Machine data (toroids and beamstrahlung) are binned in histograms based on the PLS helicity state. Laser data (photodiodes) are binned based on the CLS helicity state. Detector data (Cherenkov and PTD channels) are binned based on the CLS and PLS helicity states.

If any of the following conditions are true for a given beam crossing, the data from that beam crossing is not summed into any histogram: North electron toroid less than $100 \mathrm{ADC}$ counts (eliminates missing electron pulses), north positron toroid less than 100 ADC counts (eliminates missing positron pulses), and Cherenkov channel 9 signal greater than 200 $\mathrm{ADC}$ counts (eliminates very noisy pulses in the Cherenkov detector).

The veto conditions are selected by operators through the POLSERVER process. Operators interface with the POLSERVER process through SCPs (SLAC Control Panels). Although the default running conditions automatically activate all vetoes, it is possible for data to be accumulated with one or many of the vetoes inactive. Polarimeter runs for which the normal vetoes were inactive are removed during the analysis phase (see section 3.7).

After a designated number of accumulated beam crossings (typically 20,000), POLSERVER requests a read of the crate 3 SAM, which monitors the table position and detector voltages, and a read of an IDIM which monitors the lead pre-radiator status. Then the summary buffers and SAM data are also written to SLD data tape. The main polarimeter analysis makes use of these summary data banks.

In addition to the data acquisition described above, which is driven by beam crossing triggers, there is data taken on an hourly basis, initiated automatically. Each hour, the read and write lines of each CAMAC crate are tested with SLD crate verifiers. Additionally, the Pockels cell voltages are scanned about the nominal points, and the resulting photodiode readings are written to SLD data tape. 
There is one further complication in normal data acquisition. Since April 27, 1993, the so-called AUTOPOCKSCAN running mode was made the default running condition. In this mode, the CLS Pockels cell voltages are set away from nominal for two out of three polarimeter runs. In this manner, we effectively scan the Compton asymmetry as a function of Pockels cell voltage. These scans are fit off-line for the laser polarization as a function of the voltage settings (refer to section 3.5 for full details). The implementation of this scanning mode has enabled us to reduce the systematic error on laser polarization to below $1 \%$. In theory, all runs could be used, if properly corrected for the voltage offsets. However, the error associated with the correction is a steep function of the offset itself; therefore electron polarizations for the $A_{L R}$ analysis are calculated only using runs in which both the CP and PS Pockels cells are at their nominal voltage settings (we will hereby refer to such runs as nominal runs).

\subsection{The Energy Spectrometer}

The last hardware element encountered by electrons before they enter the beam dump is an energy spectrometer [40]. The layout of this spectrometer is shown in figure 2-23. The positron energy is measured by an identical spectrometer in the positron extraction line.

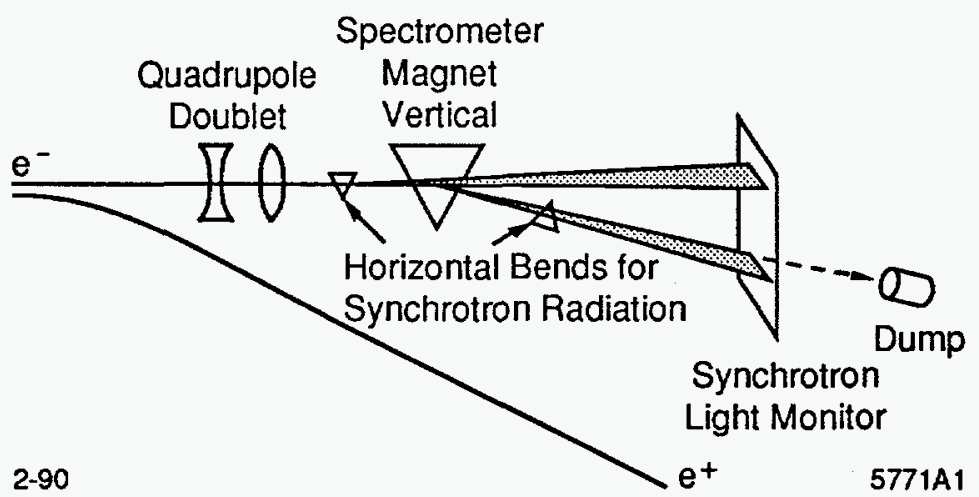

Figure 2-23: The electron extraction line energy spectrometer.

In each spectrometer, the beam passes through three dipole magnets, of which the second is a precision analysis magnet. The first and third magnets bend the beam perpendicular 
to the bend plane of the analysis magnet, and cause the beam to emit to parallel stripes of synchrotron radiation. A detector known as the WISRD (Wire Imaging Synchrotron Radiation Detector) is located approximately $15 \mathrm{~m}$ downstream of the analysis magnet. The WISRD consists of two screens of 96 copper wires each (the synchrotron stripes are detected via Compton scattering of electrons off of the wires) which measure the distance between the two synchrotron stripes. This distance (approximately $26 \mathrm{~cm}$ ) determines the deflection angle of the beam, $\delta$, through the analysis magnet which is in turn related to the beam energy, $E_{b}$ through

$$
E_{b}=\frac{c}{\delta} \int|\vec{B} \times d \vec{l}|
$$

where $B$ is the magnetic field of the analysis magnet and $d l$ lies along the beam path.

Because $A_{L R}$ is a function of center-of-mass energy, a precise measurement of this quantity is essential to the experiment. The spectrometer is capable of measuring an instantaneous energy of a single beam to within $22 \mathrm{MeV}$. Most of this error is due to electronic noise, which averages out over many pulses. Therefore the average energy of a single beam is measure to within $12 \mathrm{MeV}$ [41]. During the 1993 run, the energy spectrometer measured the average center-of-mass energy at $91.26 \pm 0.02 \mathrm{GeV}$, and the average electron beam energy at $45.64 \pm 0.01 \mathrm{GeV}$.

\subsection{The SLD Detector}

The SLC Large Detector, or SLD, was proposed in 1984 [42] and was intended to be the main detector for SLC $Z$ physics. The detector was completed in 1991 and replaced the Mark II detector at the main interaction point. A cutaway view of the detector is shown in figure 2-24. The design incorporates all the elements of modern detectors, including a precision silicon vertex detector and a Cherenkov ring imaging detector.

The $A_{L R}$ measurement is not dependent on an analysis of the $Z$ decay final states, and need not make use of the tracking and particle identification systems. It has been suggested that, for the purpose of this measurement, the entire detector could be replaced by a few 


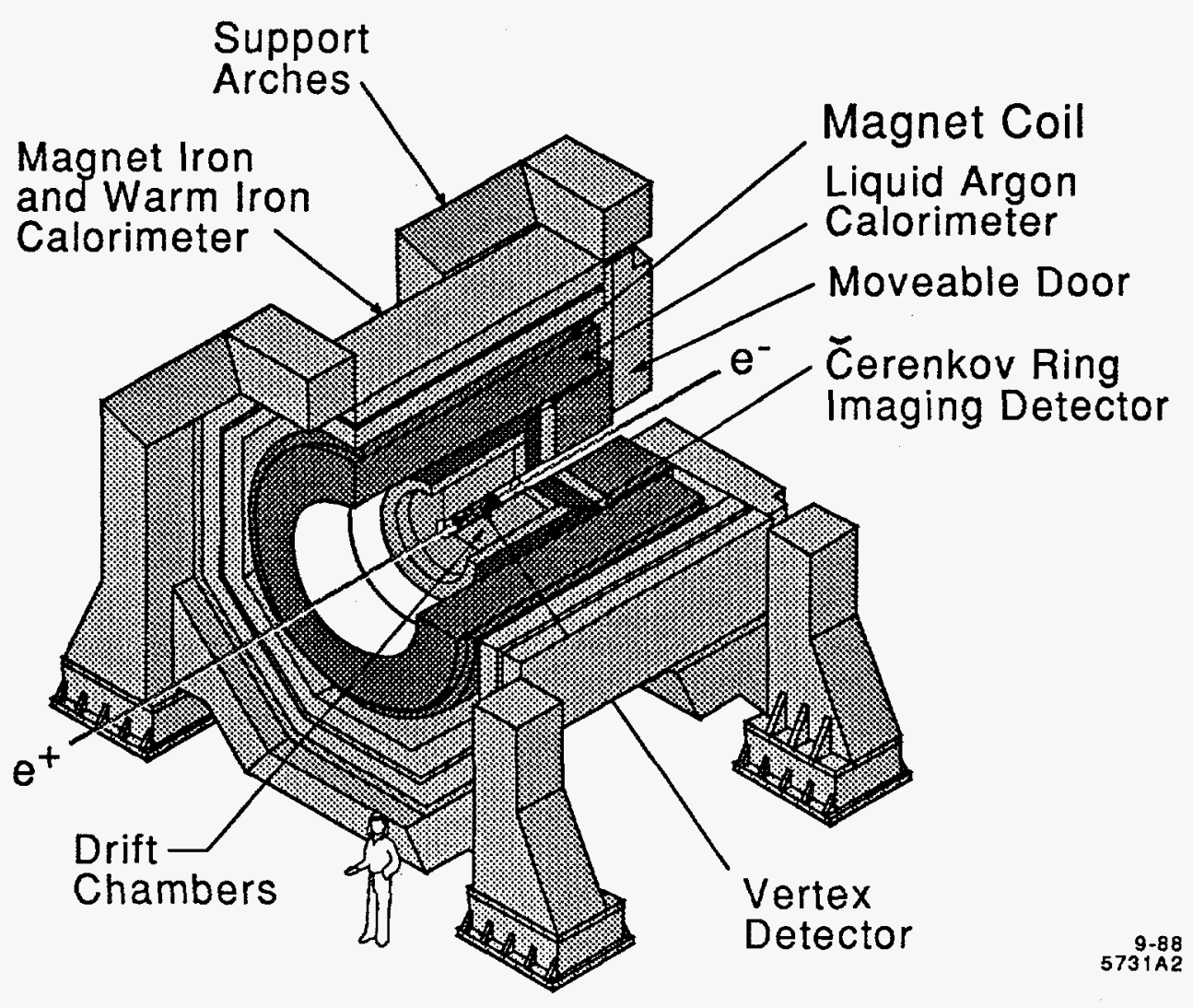

Figure 2-24: Cutaway view of the SLD detector.

phototubes. Rather than going that far, the analysis rests on one subsystem of the SLD, the liquid argon calorimeter. Given this fact, we will give a brief overview of each detector subsystem, and then a more detailed description of the liquid argon calorimeter.

\subsubsection{SLD Subsystems}

The radius of the SLD beampipe is $2.55 \mathrm{~cm}$. The SLD silicon vertex detector (VXD) [43] consists of four concentric layers of overlapping charge coupled devices (CCDs) from a radius of $2.95 \mathrm{~cm}$ to $4.15 \mathrm{~cm}$. With a total of 120 Megapixels, the VXD has a position resolution of 5 microns, which makes it a very important tool for finding secondary vertices, particularly the decay vertices of $b$ quarks. The device has an acceptance roughly equal 
to that of the central drift chamber, and data from these two systems can be combined in order to improve the overall momentum measurement.

The luminosity monitor (LUM) [44] is encountered by particles with a very small scattering angle ( $35 \mathrm{mrad}<\theta<68 \mathrm{mrad}$ ). This system has a small angle, high precision electromagnetic calorimeter at both the north and south ends of the detector. Each calorimeter consists of 23 tungsten radiator plates approximately one radiation length thick, instrumented with silicon detectors in the plate gaps. The $\mathrm{e}^{+} \mathrm{e}^{-}$scattering process is dominated by $t$-channel photon exchange at small angles, and the LUM system detects these electrons at a rate which is approximately two times the $Z$ production rate, with an energy resolution of approximately $3.0 \%$ for a $50 \mathrm{GeV}$ electron.

A particle traveling with slightly larger scattering angle ( $>700 \mathrm{mrad})$ encounters the central drift chamber (CDC). The drift chamber is a cylindrical array of sense wires, extending from a radius of $0.2 \mathrm{~m}$ to $1.0 \mathrm{~m}$. In the presence of the 0.6 Tesla solenoidal magnetic field provided by the SLD magnet, the CDC provides momentum and position measurements of charged particles. The measured momentum resolution for the $\mathrm{CDC}$ was approximately $(0.0095)^{2} / p^{2}+(0.0049)^{2}$ with a local spatial resolution of 100 microns. In addition to the CDC, the SLD is also instrumented with four endcap drift chambers (EDCs) which were to complement the $\mathrm{CDC}$ measurement in the forward region $(|\cos \theta|>0.71)$.

The next radial system is the Cherenkov Ring Imaging Device (CRID) [45], which was designed to provide particle identification over a large range of momenta. The CRID employs a hybrid system of gas $\left(\mathrm{C}_{5} \mathrm{~F}_{12}\right)$ and liquid $\left(\mathrm{C}_{6} \mathrm{~F}_{14}\right)$ Cherenkov radiators and TMAE-based TPC detectors in order to achieve the widest possible momentum acceptance. The CRID also has separate central and endcap subsystems, neither of which is yet used in physics analysis.

The SLD calorimetric system is a hybrid of lead-liquid argon and iron-gas sampling calorimeters. The inner system, the Liquid Argon Calorimeter (LAC), is described in the next section. The massive outer structure of the Warm Iron Calorimeter (WIC) [46] acts as a muon identifier and a magnetic flux return, as well as a calorimeter, and is also subdivided into central and endcap subsystems. The central system consists of eight chambers arranged 
octagonally, each chamber consisting of seven alternating steel/air-gap layers. The air gaps are instrumented with streamer tubes. The WIC system provides a $\sigma / E$ measurement of $80 \% / \sqrt{E}$, as well as some tracking information.

\subsubsection{The Liquid Argon Calorimeter}

Liquid argon sampling calorimeters have several desirable properties that lend themselves to $Z$ physics. They allow large solid angle coverage and have excellent energy resolution and uniformity of response. They may have arbitrarily fine segmentation and they offer good radiation resistance, allowing for operation close to the beamline within the magnetic fields.

The location of the SLD liquid argon sampling calorimeter with respect to the rest of the detector is most clearly shown in figure $2-25$. The LAC is composed of a cylindrical barrel calorimeter and two endcap calorimeters [47]. The barrel section covers the region of $\theta \geq 33^{\circ}(|\cos \theta| \leq 0.84)$ and is composed of 288 modules mounted in a cylindrical cryostat. In the radial direction, two separate types of modules are mounted on top of each other, the electromagnetic (EM) modules and the hadronic (HAD) modules. The endcap calorimeters cover the region $8^{\circ}<\theta<35^{\circ}(0.82>|\cos \theta|>0.99)$, and are composed of 16 wedge-shaped modules, each module also containing an EM and HAD section.

Endcap modules differ from barrel modules in design and construction, but are functionally identical, so we will describe the barrel-type module only, which is pictured in figure 2-26. The modules are constructed as parallel plate ionization chambers, with alternate planes of lead sheets and segmented lead tiles serving as the absorber, with liquid argon filling the gaps between the planes. The tile geometry forms projective towers, represented by dashed lines in figure $2-25$, which point toward the interaction point.

The LAC is longitudinally divided into four compartments, two electromagnetic followed by two hadronic. The first EM compartment contains 8 cells for a total of 6 radiation lengths $\left(6 \mathrm{X}_{0}\right)$. The second $\mathrm{EM}$ compartment contains 20 cells for a total of 15 radiation lengths. This total EM thickness contains $98 \%$ of the energy from a $50 \mathrm{GeV}$ electron. The EM sections are followed by two HAD compartments of 13 cells and 1 absorption length 


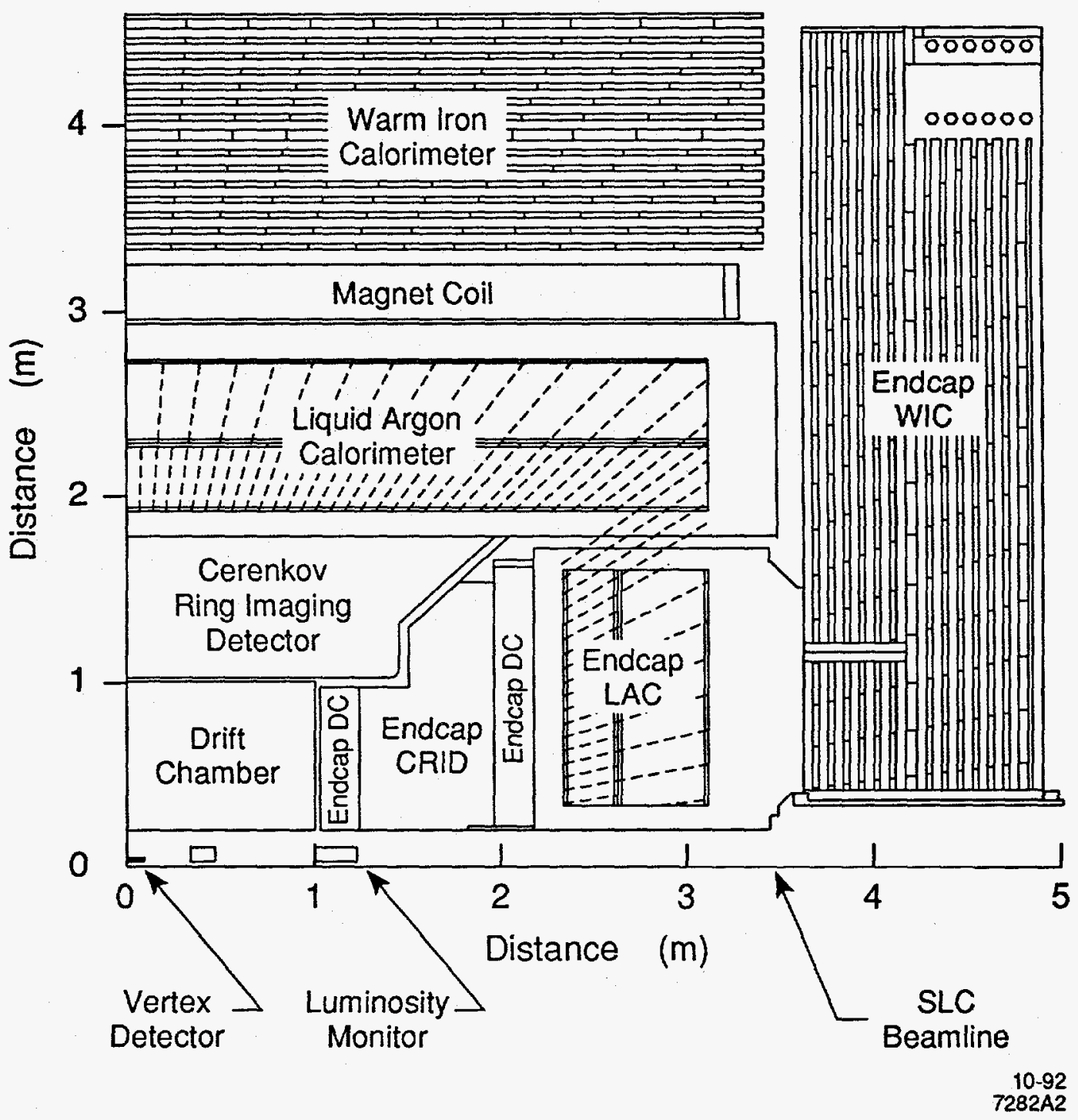

Figure 2-25: Quadrant view of the SLD detector.

(1 $\left.\lambda_{0}\right)$ each. The total thickness of the EM and HAD sections is $2.8 \lambda_{0}$, which contains 80 $90 \%$ of the energy from a hadronic shower. The LAC is divided transversely into 32,448 towers in the barrel section, 8,640 towers in the endcap, and covers the full solid-angle up to $|\cos \theta| \leq 0.98$.

The first step LAC data acquisition chain is a set of electronics called tophats, which are mounted on the detector itself and provide amplification and digitization of the tower 


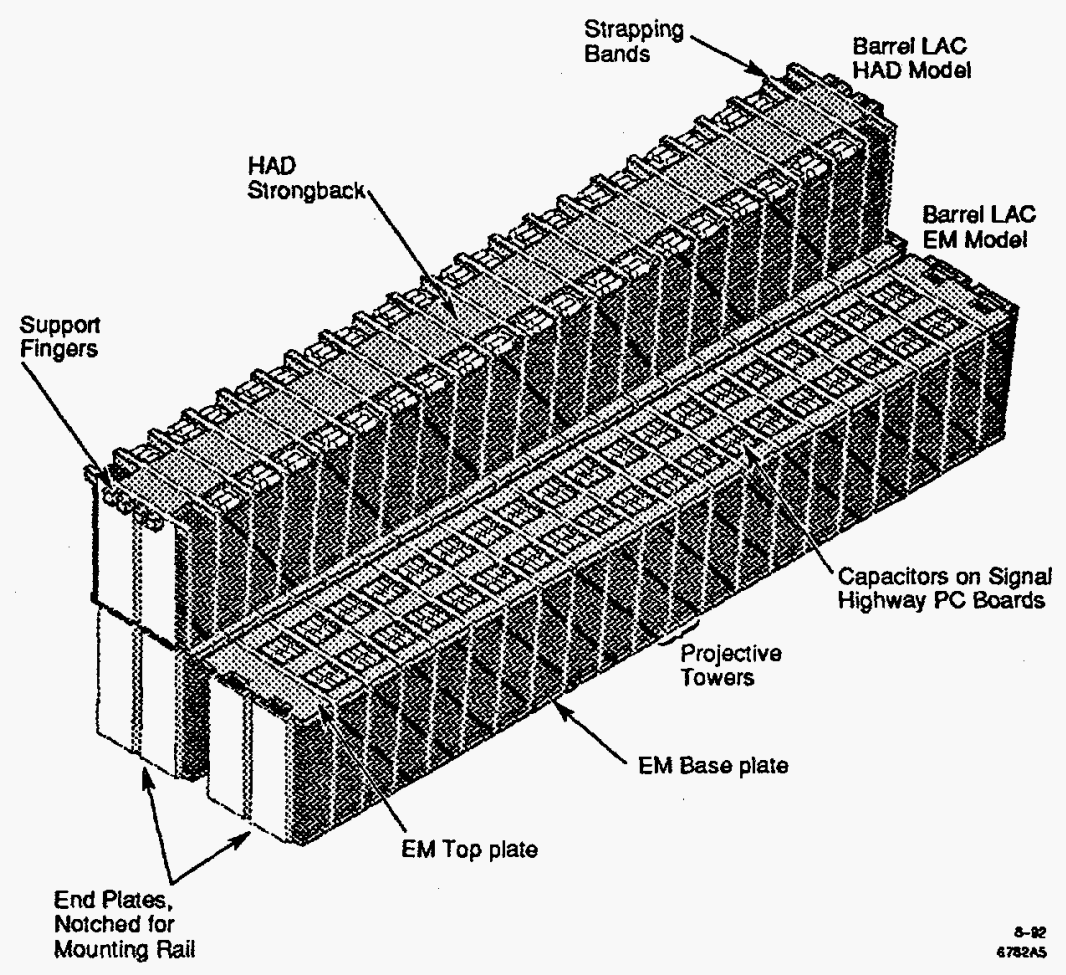

Figure 2-26: LAC barrel EM and HAD modules

data. This data is read out through fiber optic connections by 32 FASTBUS slave modules, under the coordination of one master module. If an event is triggered, the FASTBUS modules send the information to the SLD data acquisition VAX. One process places the event into a shared pool, after which a second process writes the entire data record to tape.

The subsequent off-line analysis of LAC data is the subject of Chapter 4. 


\section{Chapter 3}

\section{Compton Polarimetry}

Electron polarization is the most unique and important aspect of the $A_{L R}$ experiment and also contributes the largest systematic uncertainty to the experiment. The Compton Polarimeter provides a precise monitor of the longitudinal beam polarization through the wellunderstood QED process of Compton scattering. This chapter will address the details of Compton scattering kinematics, polarization extraction (polarimetry), polarimeter data selection, and systematic errors.

\subsection{Compton Scattering Kinematics}

The elastic scattering of an electron and photon, known as Compton scattering, is described, to lowest order in QED, by an $s$-channel and $u$-channel electron exchange, as illustrated in figure 3-1.

As was previously described, the energy of the photon in the laboratory frame is $2.33 \mathrm{eV}$, while the energy of the electron is $45.6 \mathrm{GeV}$. This makes working in the laboratory frame somewhat cumbersome. However, the analyzing magnets and polarimeter detectors live in the laboratory frame, so we require cross sections in this frame. We will start with the known cross section in the rest frame of the electron and then outline the transformation of variables from the rest frame to the laboratory frame. 


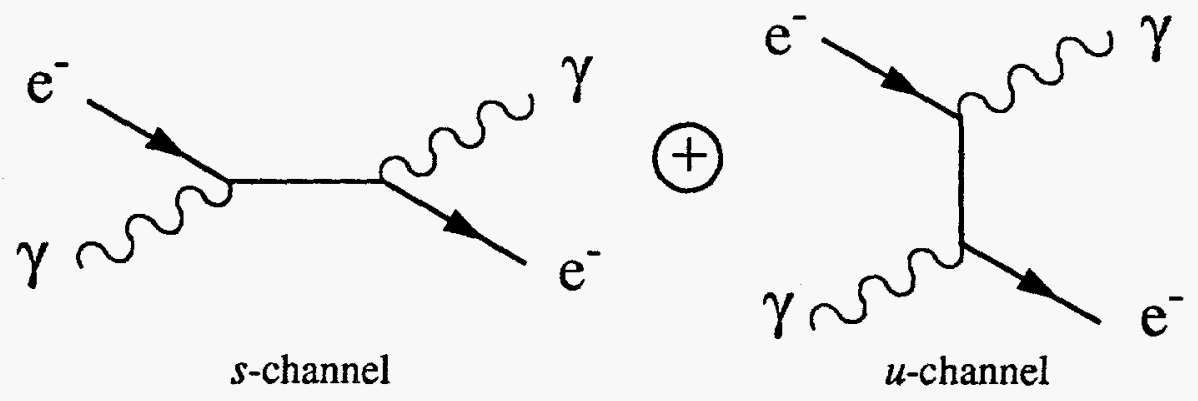

Figure 3-1: Tree-level Compton scattering.

The differential cross section in the rest frame of the electron is given by [48]

$$
\left(\frac{d \sigma}{d \Omega}\right)=\frac{1}{2} r_{0}^{2}\left(\frac{k^{\prime}}{k}\right)\left[\frac{\left(k-k^{\prime}\right)^{2}}{k k^{\prime}}+1+\cos ^{2} \theta_{0}\right]\left\{1-P_{\gamma} P_{e} A_{e \gamma}\left(\vec{k}, \vec{k}^{\prime}\right)\right\}
$$

where $r_{0}$ is the classical radius of the electron, $k$ and $k^{\prime}$ are the momenta of the incident and scattered photons, and $\theta_{0}$ is the photon scattering angle. $P_{\gamma}$ is the circular polarization of the photon (only photon helicity states couple to the electron polarization), $P_{e}$ is the polarization of the electron, and $A_{e \gamma}$ is the asymmetry function, defined below. Note that $P_{\gamma}$ and $P_{e}$ are signed quantities. We use the convention that either $P$ is positive when the angular momentum is aligned with the momentum of the particle. Such states are designated righthanded or positive helicity states throughout this document, and are labelled by an " $R$ ". The opposite case, when the spin or angular momentum is anti-parallel to the momentum, is referred to as the left-handed or negative helicity state, and is labelled by an " $L$ ".

The asymmetry function $A_{e \gamma}$ is defined by

$$
A_{e \gamma}\left(\vec{k}, \overrightarrow{k^{\prime}}\right)=\frac{\left(\frac{1}{k^{\prime}}-\frac{1}{k}\right)\left[\vec{k} \cos \theta_{0}+\vec{k}^{\prime}\right] \cdot \hat{s}}{\frac{\left(k-k^{\prime}\right)^{2}}{k k^{\prime}}+1+\cos ^{2} \theta_{0}}
$$

This function is the scalar product of a linear combination of the photon momenta and the electron spin direction, $\hat{s}$. This allows for the possibility of three-axis polarimetry, which is difficult with the present Compton polarimeter geometry, because one is required to 
detect the scattered photons, which diverge only minimally from the beamline. Nevertheless, research and development for a three-axis Compton polarimeter at SLAC is already underway. The 1993 Compton polarimeter, which detects only scattered electrons, is only useful for longitudinal polarization measurements, a point which is discussed in section (3.2.2) below.

\subsubsection{Laboratory Frame Scattering Kinematics}

We define $E$ and $K$ to be the incident energies of the electron and photon, and $E^{\prime}$ and $K^{\prime}$ to be the scattered energies of the electron and photon. We now introduce the Compton scattering $y$ parameter which is the ratio of the minimum scattered electron energy to the incident electron energy:

$$
\begin{aligned}
y & \equiv\left(1+\frac{4 K E}{m^{2}}\right)^{-1} \\
K_{\text {max }}^{\prime} & =E(1-y) \\
E_{\text {min }}^{\prime} & =E y
\end{aligned}
$$

The electron has it's minimum energy in the case of complete backscattering in the centerof-mass frame. The emission angle of the scattered photon is then related to the scattered photon energy through the following expression

$$
K^{\prime}=K_{\max }^{\prime}\left[1+y\left(\frac{E \theta_{K}}{m}\right)^{2}\right]^{-1} \equiv K_{\text {max }}^{\prime} \cdot x
$$

wherein the kinematic variable $x$ is defined. The values of $x$ range from zero (no scattering) to one (full backward scattering, the kinematic endpoint).

The transformation between the electron rest frame and the laboratory frame variables is defined through the following equations:

$$
\begin{aligned}
& k=\frac{2 E}{m} K \\
& x=\frac{K^{\prime}}{K_{\text {max }}^{\prime}}=\frac{1-\cos \theta_{0}}{2 y+(1+y)\left(1-\cos \theta_{0}\right)}
\end{aligned}
$$


Applying this transformation to equations (3.1) and (3.2) yield the expression for the cross section in the laboratory frame, in terms of the laboratory variables $x$ and $y$ :

$$
\left(\frac{d^{2} \sigma}{d x d \phi}\right)_{C o m p}=\left(\frac{d^{2} \sigma}{d x d \phi}\right)_{\text {unpol }}\left\{1-P_{\gamma}\left[P_{e}^{z} A_{e \gamma}^{z}(x)+P_{e}^{t} \cos \phi A_{e \gamma}^{z}(x)\right]\right\}
$$

where $\phi$ is the is the azimuthal angle of the photon with respect to the transverse polarization component of the electron, and the superscripts $z$ and $t$ refer to the longitudinal and transverse polarization components, respectively. The unpolarized cross section is given by

$$
\left(\frac{d^{2} \sigma}{d x d \phi}\right)_{\text {unpol }}=r_{0}^{2} y\left[\frac{x^{2}(1-y)^{2}}{1-x(1-y))}+1+\left(\frac{1-x(1+y)}{1-x(1-y)}\right)^{2}\right]
$$

and the longitudinal and transverse asymmetries are defined by

$$
\begin{gathered}
A_{e \gamma}^{2}=r_{0}^{2} y[1-x(1+y)]\left[1-\frac{1}{[1-x(1-y)]^{2}}\right] \cdot\left(\frac{d^{2} \sigma}{d x d \phi}\right)_{\text {unpol }}^{-1} \\
A_{e \gamma}^{t}=r_{0}^{2} y x(1-y)\left[\frac{\sqrt{4 x y(1-x)}}{1-x(1-y)}\right] \cdot\left(\frac{d^{2} \sigma}{d x d \phi}\right)_{\text {unpol }}^{-1}
\end{gathered}
$$

Figure 3-2 shows these asymmetries and the cross section plotted as a function of the kinematic variable $x$.

\subsubsection{Radiative Corrections}

The first-order radiative corrections to polarized Compton scattering have been calculated [49]. The effects are less than $0.3 \%$ on the unpolarized cross section and less than $0.1 \%$ on the asymmetry of the outer channels. It is conceivable that these corrections will become important in a future analysis that would use the full Compton spectrum and attempt to measure the polarization to within $0.5 \%$. However, radiative corrections are ignored in the 1993 analysis. 


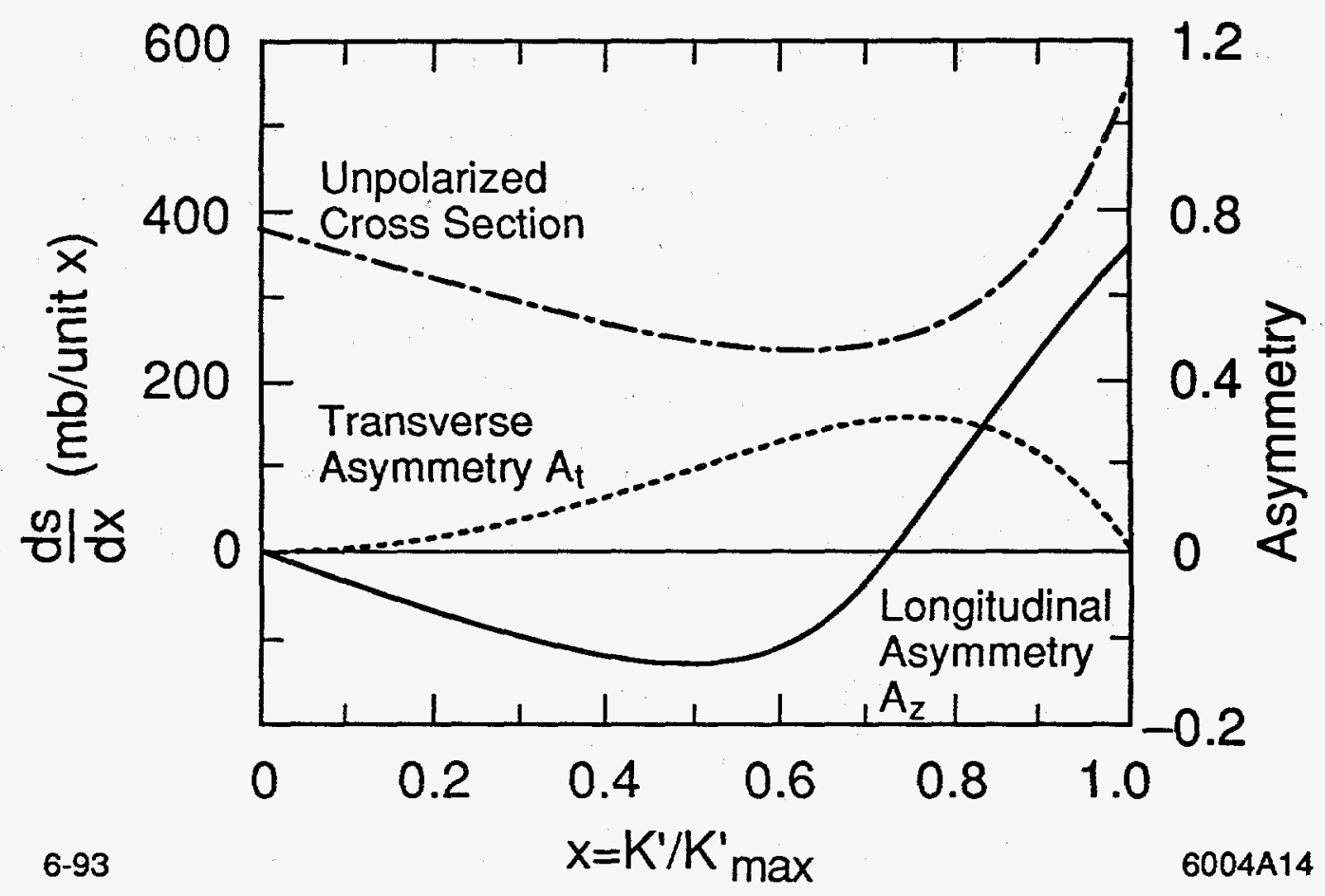

Figure 3-2: The Compton cross section and asymmetries.

\subsection{Extracting the Beam Polarization from Compton Scattering}

The remainder of this chapter deals with the specifics of Compton polarimeter analysis, including the bend magnets, the target, and the detectors. The first detailed account of Cherenkov detector analysis appears in Reference [50]. An updated account for the 1993 run follows, much of which is derived from the content of Reference [51].

We have shown that the QED process of Compton scattering has an intrinsic asymmetry and that the cross section can be separated into polarized and unpolarized parts. In this section we discuss how the analyzing bend magnet of the polarimeter translates an asymmetry as a function of energy into an asymmetry as a function of distance. We then describe how this new function is measured as an experimental asymmetry in a detector. 


\subsubsection{The Analyzing Bend Magnet}

In the laboratory frame, Compton electrons are scattered very much in the forward direction. The maximum electron scattering angle of $9.1 \mu$ radians is considerably less than the beam divergence. Thus the Compton scattered electrons travel colinearly with the main electron beam until they pass through the first dipole bending magnets (B31 and B32, the so-called soft and hard bending magnets), the combination of which is referred to as the analyzing bend magnet of the polarimeter. These magnets provide a transverse momentum kick to the electrons and thereby couple the scattered electron energy $E$, with a spatial distance $s$, measured perpendicularly from the infinite momentum line, through the following relation

$$
s=\frac{B D}{E}=\frac{B D}{E_{b}[1-x(1-y)]}
$$

where $B$ is the effective bend strength of the two magnets, $D$ is the distance from the effective bend center of the magnets along the neutral beam line from which $s$ is measured, $E_{b}$ is the beam energy, and $x$ and $y$ are the kinematic parameters defined in section 3.1.1. In the case of the polarimeter, $s$ is measured at the entrance of the Cherenkov detector channels. For the nominal beam and target energies, $y=0.3804$.

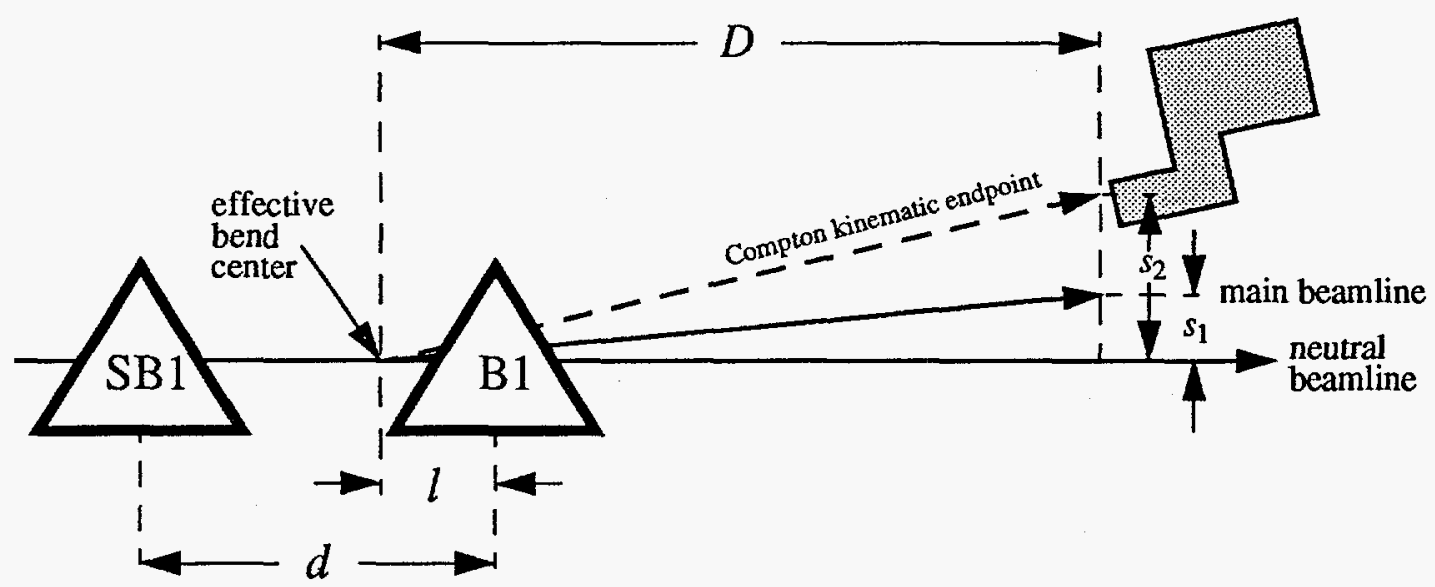

Figure 3-3: The analyzing bend magnet of the Compton polarimeter. 
The geometry of the analyzing bend magnet is clarified in figure 3-3 (the angles and distances are not drawn to scale). The design bend angle of magnet SB1 is $1.0 \mathrm{mrad}$. The design bend angle of magnet B1 is $17.27 \mathrm{mrad}$. The distance $d$ between the bend centers of the magnets is $385.7 \mathrm{~cm}$. Thus the effective bend center occurs at a distance $l=22.3 \mathrm{~cm}$ from the center of B1. The effective bend angle of the combined analyzing magnet is $18.27 \mathrm{mrad}(833.2 \mathrm{MeV} / \mathrm{c})$. The distance from the effective bend center to the face of the detector, $D$, has been measured to be $355.8 \mathrm{~cm}$. Therefore $s_{1}$ (the distance from the neutral beamline to the main beamline) is $(B D) / E_{b}=6.50 \mathrm{~cm}$, and $\mathrm{s}_{2}$ (the distance from the neutral beamline to the Compton kinematic endpoint) is $(B D) /\left(E_{b} y\right)=17.08 \mathrm{~cm}$.

The combination of these numbers with equation (3.10) gives us the transformation Jacobian necessary to go from the cross section as a function of the energy variable $x$ to the cross section as a function of the space variable $s$ :

$$
\frac{d \sigma}{d s}=\left(\frac{d \sigma}{d x}\right) \cdot\left(\frac{d x}{d s}\right), \quad \frac{d x}{d s}=\frac{B D}{E_{b}(1-y) s^{2}}
$$

where the differential cross section with respect to $x$ is fully defined by equations (3.6) through (3.9).

\subsubsection{The Experimental Asymmetry}

Because the laboratory scattering angles are so small in the Compton polarimeter, all scattered electrons are swept into the same energy-space correlation by the bending magnets, regardless of their azimuth. Thus the detector effectively integrates over the azimuthal distribution and eliminates the transverse asymmetry term in the cross section. We simplify the expression for the cross section even further by absorbing the unpolarized cross section (including $d x / d s$ ) into the function $U(s)$, and the remaining polarization-dependent part into the function $A(s)$, writing

$$
\left(\frac{d \sigma}{d s}\right)_{\text {Compton }}=U(s)\left[1+P_{\gamma} P_{e}^{C} A(s)\right]
$$

where $s$ is the transverse spatial coordinate at the detector. 
It follows from (3.12) that the observed or measured asymmetry in a given channel $i$, $A_{i}^{m}$, is directly related to the product of the electron and photon polarizations

$$
\begin{aligned}
& A_{i}^{m}=\frac{N_{i}^{L L}+N_{i}^{R R}-N_{i}^{L R}-N_{i}^{R L}}{N_{i}^{L L}+N_{i}^{R R}+N_{i}^{L R}+N_{i}^{R L}}=P_{\gamma} P_{e}^{C} a_{i} \\
& \text { where } N_{i}^{X Y}=O_{i}^{X Y}-B_{i}^{X}
\end{aligned}
$$

The number of observed Compton electrons in channel $i, N_{i}^{X Y}$, is equal to the average number of counts observed in the channel in the given helicity combination $\mathrm{XY}, O_{i}^{X Y}$, minus the background observed in that channel when the laser is off, $B_{i}{ }^{X}$. Note that the background term does depend on the electron helicity state; this takes into account any background-related asymmetry. The two superscripts refer to the helicity state of the electrons and photons respectively. The $\mathrm{LR}$ and $\mathrm{RL}$ states corresponds to a $J_{3 / 2}$ spin combination, when the electron and photon angular momenta are parallel. The LL and RR states represents the $\mathrm{J}_{1 / 2}$ combination, where the photon and electron angular momenta are anti-parallel. The $\mathrm{J}_{3 / 2}$ case is the larger cross section in Compton scattering [52]. $P_{\gamma}$ is the circular polarization of the laser, $P_{e}{ }^{C}$ is the electron polarization measured at the Compton interaction point, and $a_{i}$ is the analyzing power of channel $i$.

The analyzing power is defined by the following expression

$$
a_{i}=\frac{\int U(s) A(s) \rho_{i}(s) d s}{\int U(s) \rho_{i}(s) d s}
$$

where $\rho$ is response function (acceptance as a function of $\mathrm{s}$ ) for channel $i$. The analyzing power of a channel is the average of the Compton asymmetry, weighted by the unpolarized cross section (which is relatively flat across the detector acceptance) and the detector response function, $\rho$, for that channel. If we recast equation (3.13), we see that the measurement of the beam polarization, $P_{e}{ }^{C}$, rests on the measurement of the experimental 
asymmetry, the determination of the analyzing powers, and the measurement of the laser polarization.

$$
P_{e}^{C}=\frac{A_{i}^{m}}{a_{i} P_{\gamma}}
$$

The quantities on the right hand side of equation (3.15) are the topics of the following sections.

\subsection{Measuring the Experimental Asymmetry}

This section describes four potential problems facing the experimental asymmetry measurement: contamination by other asymmetries such as photon and/or electron current asymmetries, the effects of non-linear detector response, the effects of signal-correlated background noise, and the effects of saturated detector pulses.

\subsubsection{Compton Asymmetries}

Measurement of the experimental asymmetry rests on a proper accounting of the four Compton asymmetries that affect the observed asymmetry: the left-right asymmetries in the electron and photon currents, and the left-right polarization asymmetries of the electron and photon beams. We define these asymmetries (the $\Delta$ quantities) through the following relations:

$$
\begin{aligned}
P_{e}^{L}=\left(1+\Delta P_{e}\right) P_{e}^{C} & P_{e}^{R}=\left(1-\Delta P_{e}\right) P_{e}^{C} \\
P_{\gamma}^{L}=\left(1+\Delta P_{\gamma}\right) P_{\gamma}^{C} & P_{\gamma}^{R}=\left(1-\Delta P_{\gamma}\right) P_{\gamma}^{C} \\
I_{e}^{L}=\left(1+\Delta I_{e}\right) I_{e} & I_{e}^{R}=\left(1-\Delta I_{e}\right) I_{e} \\
I_{\gamma}^{L}=\left(1+\Delta I_{\gamma}\right) I_{\gamma} & I_{\gamma}^{R}=\left(1-\Delta I_{\gamma}\right) I_{\gamma}
\end{aligned}
$$

It can be shown that the measured asymmetry $A_{i}^{m}$ defined in the previous section is independent of all the $\Delta$ quantities defined above, to first order in those asymmetries. Therefore the measured asymmetry $A_{i}^{m}$ is the correct quantity to use in the $A_{L R}$ analysis, as described in section 1.5.1 However, we are also interested in making corrections to the 
assumption that the polarization asymmetry is zero, in which case it is necessary to use more polarimeter information.

It can also be shown that the "asymmetry asymmetries" in a given channel $i$ are related to the $\Delta$ quantities in the following manner:

$$
\begin{aligned}
& \frac{N_{i}^{L L}-N_{i}^{R L}}{N_{i}^{L L}+N_{i}^{R L}}-\frac{N_{i}^{R R}-N_{i}^{L R}}{N_{i}^{R R}+N_{i}^{L R}}=2\left[\Delta I_{e}+A_{i}^{e} \Delta P_{\gamma}-A_{i}^{e} A_{i}^{e}\left(\Delta I_{e}+\Delta P_{e}\right)\right] \\
& \frac{N_{i}^{L L}-N_{i}^{L R}}{N_{i}^{L L}+N_{i}^{L R}}-\frac{N_{i}^{R R}-N_{i}^{R L}}{N_{i}^{R R}+N_{i}^{R L}}=2\left[\Delta I_{\gamma}+A_{i}^{e} \Delta P_{e}-A_{i}^{e} A_{i}^{e}\left(\Delta I_{\gamma}+\Delta P_{\gamma}\right)\right]
\end{aligned}
$$

Each channel makes an independent measurement of the quantities defined in equations (3.16) and (3.17). There are four unknowns, and therefore a combination of measurements from two channels is sufficient to extract the Compton asymmetries through a system of linear equations. Channels 1 and 6 were used in the 1993 analysis for this purpose; channel 6 was used because it was the best understood of the outer channels, and channel 1 was included because the greatest statistical power is obtained by using two channels whose measured asymmetries are maximally different. Note that the determination of the $\Delta$ quantities is independent of the analyzing powers and laser polarization.

The $\Delta$ quantities are calculated, along with the measured asymmetry, $A_{i}^{m}$, for each channel, for every nominal polarimeter run. These numbers are luminosity weighted by associating each $Z$ event with the measurement made closest in time to that event, and averaging the resulting list of measurements. The results are shown in figure 3-4. Of particular interest is the electron polarization asymmetry, measured to be

$$
\Delta P_{e}=(-3.3 \pm 0.1) \times 10^{-3}
$$

\subsubsection{Detector Linearity}

The Cherenkov detector phototubes employ a special base design that allows one to vary the voltage on the photocathode, and thereby vary the gain, while maintaining a con- 

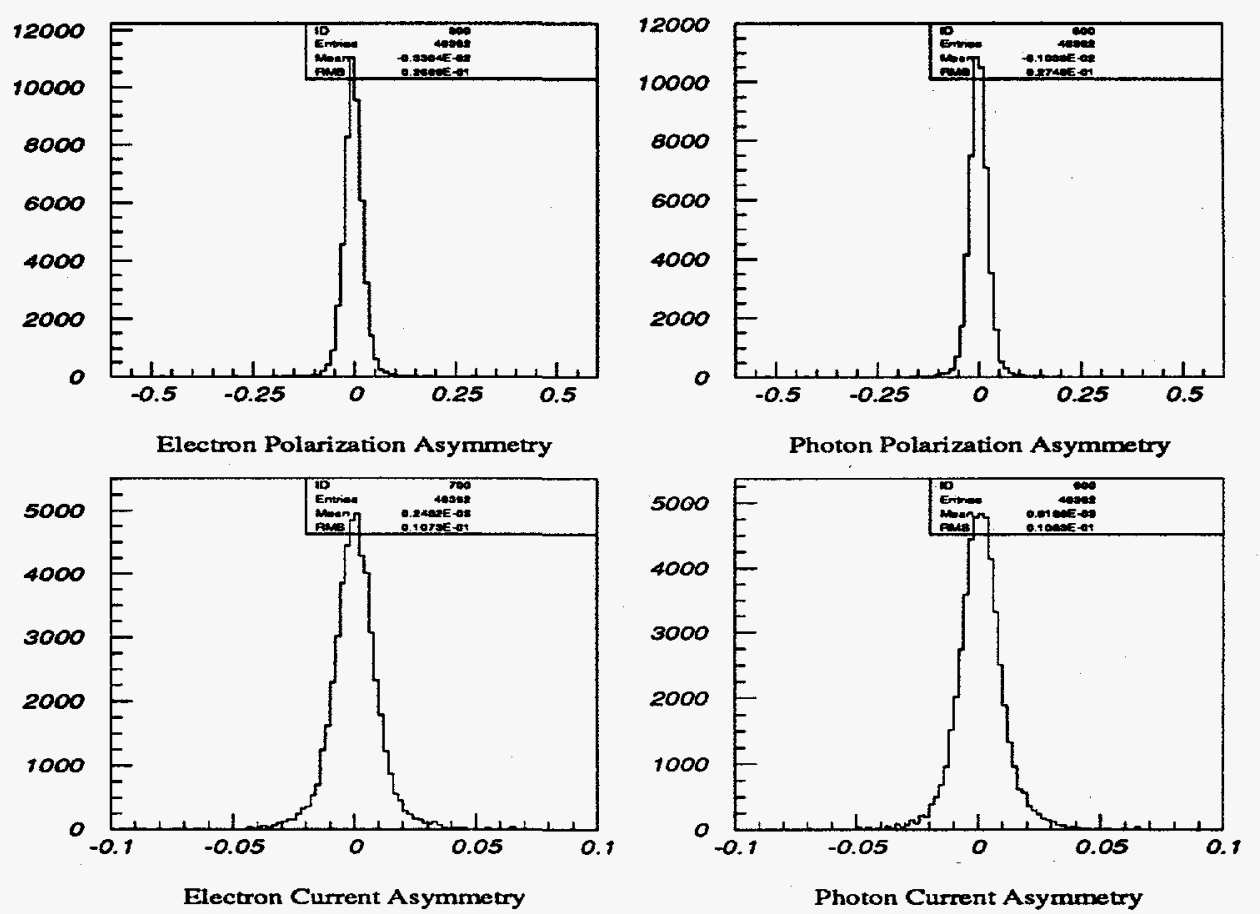

Figure 3-4: Luminosity-weighted Compton Asymmetries

stant voltage on the latter dynode stages, thereby avoiding any space charge effects that alter the saturation characteristics of the tube. Futhermore, the linearity is calibrated with the Compton signal itself, thereby removing any uncertainty associated with differences in a laboratory based calibration versus the true response in the accelerator environment.

The linearity of Cherenkov channel 6 was measured by varying the gain of the photocathode and comparing the measured asymmetry to that in channel 7 , whose signal height was constant throughout the measurement. Results of this experiment are shown in figure 3-5, where the normalized asymmetry, $A^{m}{ }_{6} / A^{m}{ }_{7}$, is plotted versus the pedestal subtracted signal height for the average $\mathrm{J}_{3 / 2}$ (largest signal) state, $\mathrm{PH}_{6}^{+}$, where $\mathrm{PH}^{+}$is defined by

$$
P H^{+}{ }_{i}=\frac{\left(O_{i}^{L L}+O_{i}^{R R}\right)}{2}-\text { Pedestal }_{i}
$$




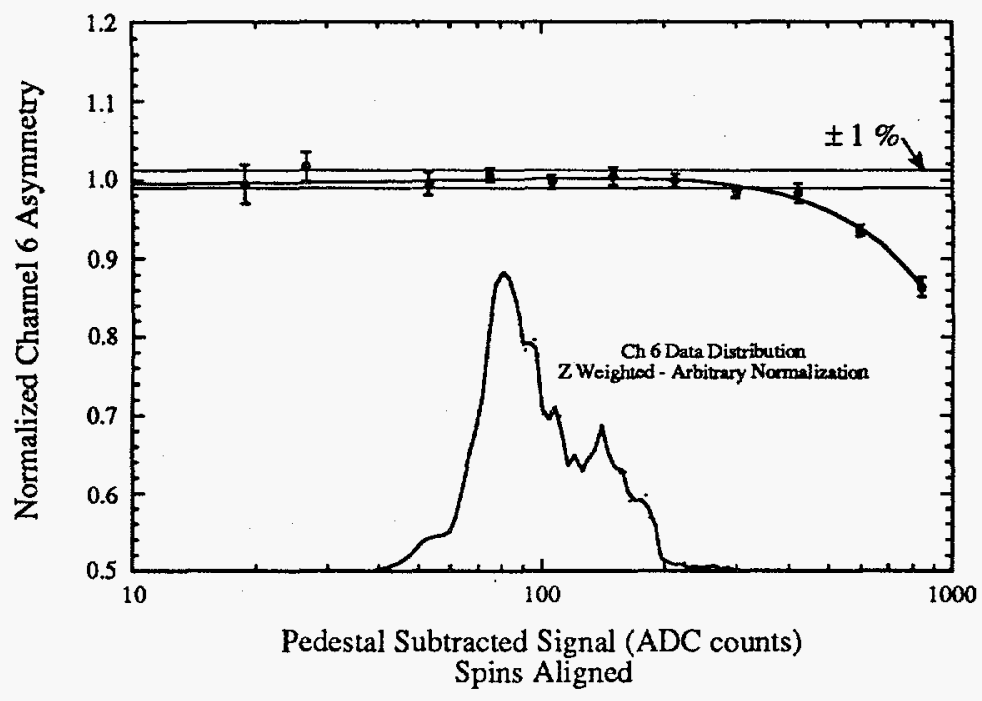

Figure 3-5: Cherenkov Channel 6 Linearity Calibration

for the observed signal plus background in the given photon/electron helicity state, $O^{X Y}$, in channel $i$. Also plotted in figure 3-5 is a histogram of the average $\mathrm{PH}_{6}^{+}{ }_{6}$ for every 1993 polarimeter run, which indicates that nearly all of the 1993 data is within the very linear response range of the phototube. Data outside the range of $\mathrm{PH}_{6}^{+}{ }_{6}>145$ counts is corrected based on the best-fit curve shown. A systematic error of $0.7 \%$ is associated with the uncertainty of the channel 6 linearity calibration.

Once the linearity of channel 6 has been characterized, it is possible to determine the linearity of channel 7 by comparing the channel 7 asymmetry to the corrected channel 6 asymmetry, over the entire polarimeter data sample. The results are shown in figure 3-6. A significant non-linearity is evident in the channel 7 response. To check the testing procedure, the same procedure is applied to channels 4 and 5 , which are seen to be quite linear. Thus the channel 7 non-linearity is a real effect, not an artifact of an inadequate calibration procedure. In any case, deviations from linearity lie within the $\pm 1 \%$ band over the range of channel 7 signals. The asymmetries are corrected to the best-fit curve for all signal heights, and a systematic uncertainty of $0.7 \%$ due to non-linearity is also assigned to channel 7 . 


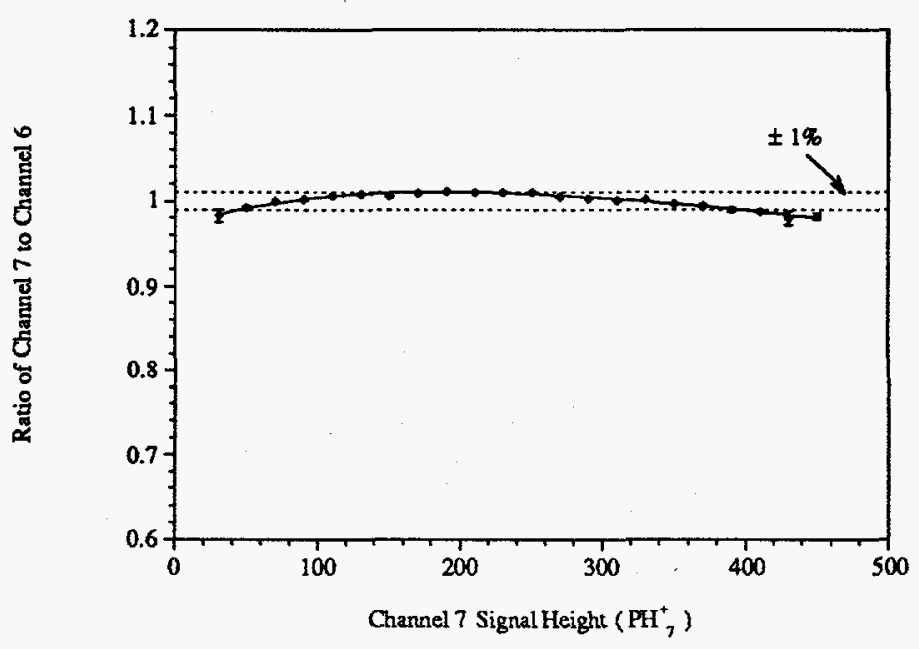

Figure 3-6: Cherenkov Channel 7 Linearity Calibration

\subsubsection{Electronic and Laser Noise Pickup}

It was observed during the 1992 SLD run that the firing of the laser Q-switch electronics induces approximately one count of signal in the Cherenkov detector ADCs. This pickup occurs only when the laser is fired, and must be subtracted to avoid diluting the measured asymmetries. The effect is especially significant when the signal itself is small.

The laser noise pick-up is monitored continually throughout the run, using raw data pulses from when the laser was fired but the electron beam was missing. The electron beam toroids included in the Compton data stream reliably identify these missing electron pulses. There were in fact 29 polarimeter runs in 1993 during which the electron toroids were not working. These runs were identified by plotting $\mathrm{PH}_{6}^{+}$versus all toroid signals less than 100 counts. Runs in which $\mathrm{PH}_{6}^{+}$was greater than 5 counts under these circumstances were removed from the analysis.

For the remaining runs in which the electron toroids were operational, the laser noise pick-up correction was determined for each channel, approximately once per hour. Each nominal polarimeter background is adjusted with the laser noise correction associated most closely in time with the polarimeter run. The time history of this correction is shown in figure 3-7. 


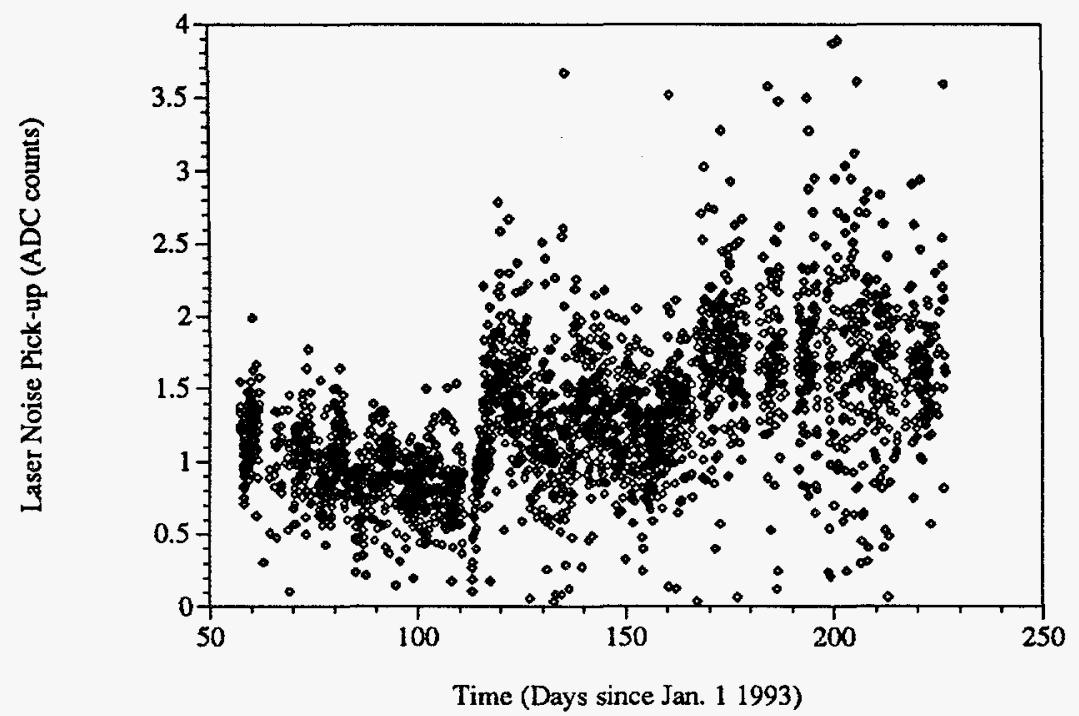

Figure 3-7: A time history of the laser noise pick-up for Cherenkov channel 6.

From the local spread of the measurements, we infer a statistical error of approximately 0.3 counts on any given correction. However, the ultimate number of significance in the $A_{L R}$ analysis is the average polarization. This means that only the average correction directly affects the error on $A_{L R}$, and the error on the average correction shrinks with the number of measurements, which is on the order of 3000 . Therefore the error on the laser noise pick-up correction has a negligible contribution to the overall systematic error, once the correction is applied.

Electronic noise arising from channel-to-channel cross-talk has been studied in two ways: first by searching for signal in all other tubes when only one tube has voltage applied, and second by searching for signal in one unenergized tube when all other tubes have a voltage applied. The first study showed that no unenergized tube measured more than $0.1 \%$ of the signal from the energized channel. The complementary study showed no measurement greater that $0.5 \%$ of the normal signal in an unenergized tube induced by cross-talk from all other energized tubes. However, the effect of such cross-talk on the measured asymmetry in a given channel is much smaller. We therefore assign a conservative estimate of $0.2 \%$ systematic uncertainty due to electronic noise. 


\subsubsection{Saturated Pulses}

At first consideration, it seems that pulses that saturate or nearly saturate the Cherenkov ADCs should be of some concern. Such pulses are either extraneous noise unrelated to the Compton signal, or unusually large Compton signals that drive the phototubes into their non-linear regions. Either case might be expected to bias the average asymmetry for the run in which the anomalous pulses are included. The Cherenkov channel 9 threshold veto vetoes any pulse in which the signal in channel 9 is greater than 200 ADC counts. A study of the raw data shows that this veto does remove a large percentage of anomalously large pulses from channels 6 and 7, but not all. Out of a sample 8 million channel 7 pulses, approximately 30 pulses above $1000 \mathrm{ADC}$ counts survive the channel 9 veto.

However, a Monte Carlo study of the saturation effect shows that, even though some saturated pulses may pass the veto, they do not bias the average asymmetry measurement, as long as such pulses occur on signal and background pulses with equal probability. This condition is assured by the $11 \mathrm{~Hz}$ operation of the target laser, which is unlikely to be correlated with any periodicity and randomly samples all 120 machine time slots.

The reason for the non-effect is obvious upon some consideration: the slight decrease in the measured asymmetry induced by saturated signal pulses is exactly cancelled by an increase in the measured asymmetry induced by saturated background pulses. On average, there is no asymmetry bias, and the saturated pulses merely inflate the statistical error of the run in which they are included. Results of the Monte Carlo showing the average ratio of the measured asymmetry to the true asymmetry as a function of the number of saturated pulses per run are shown in figure 3-8.

\subsection{Determination of the Analyzing Powers}

Equation (3.14) shows that in order to determine the analyzing power of a given channel, it is essential to understand the response function $\rho$ of the detector. Also implicit in this equation is the necessity of knowing the limits on the integrals, which is to say, to know where the detector channels are located relative to the Compton spectrum. The problem of 


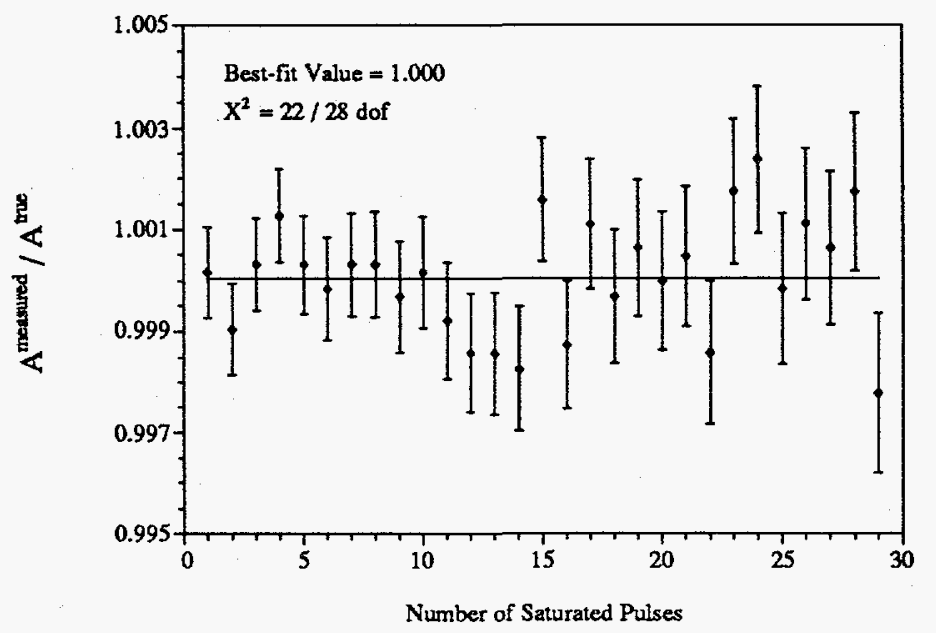

Figure 3-8: Monte Carlo results showing the effect of saturated pulses.

locating the detector relative to the scattering spectrum is referred to as the detector endpoint calibration.

\subsubsection{Endpoint Calibration}

The platform which supports the Cherenkov detector is mounted on bearings and can thereby be moved perpendicular to the beamline with a remotely controlled stepping motor. The position of the platform is monitored by a precision linear potentiometer. The nominal position of the platform is fixed by a physical stop and is additionally monitored by a stepping motor limit switch.

The analyzing powers in the outer channels depend primarily on the location of the detector with respect to the Compton scattering spectrum. This location is determined by scanning the detector across the spectrum, observing the fall-off of the signal, and comparing this response to a Monte Carlo simulation. The signal is normalized to an inner channel to remove any luminosity fluctuations from the scan. The change in the cross section seen by each channel is taken into account by the Monte Carlo, which also includes the effect of the detector response function $\rho$.

The endpoint was scanned three times during the 1993 run. An example of such a scan is shown in figure 3-9. The uncertainty on the determination of the edge position on a given 


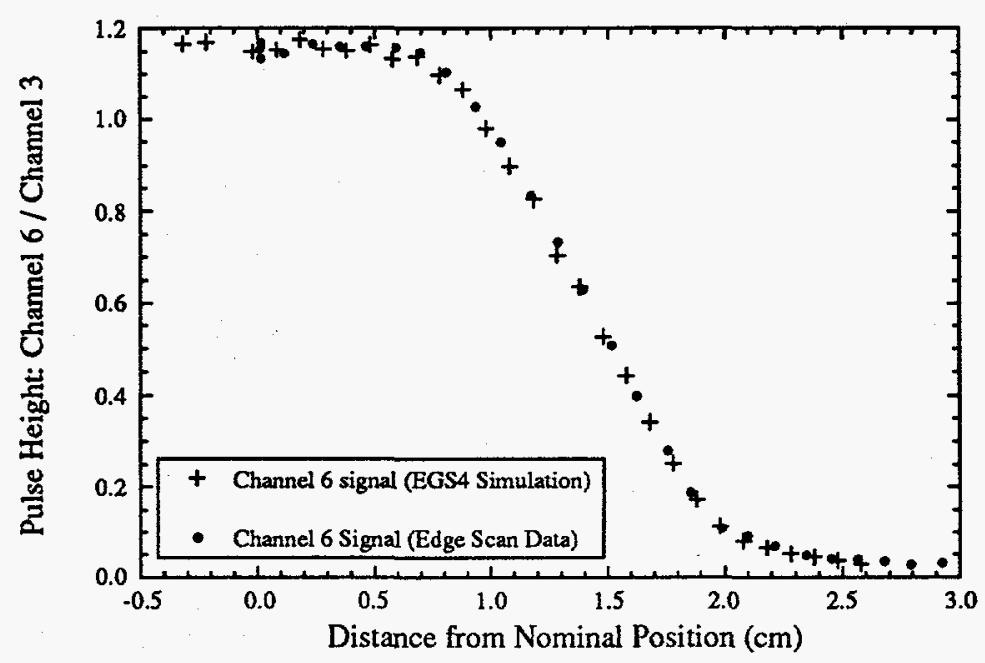

Figure 3-9: Compton kinematic edge scan.

\begin{tabular}{|c|c|}
\hline Date & Edge Position $(\mathrm{cm})$ \\
\hline \hline $4 / 26 / 93$ & $0.86 \pm 0.04$ \\
\hline $5 / 02 / 93$ & $0.84 \pm 0.02$ \\
\hline $7 / 15 / 93$ & $0.87 \pm 0.02$ \\
\hline
\end{tabular}

Table 3-1: 1993 Endpoint Scan Results

scan is less than 200 microns. The results of the three scans are shown in table 3-1; the quoted position is the distance from the kinematic endpoint to the inner (closest to the beam line) wall of channel 7.

\subsubsection{Zero Asymmetry Point}

It is not sufficient to determine the location of the endpoint at specific times during the run; we also require a relative position monitor throughout the run in order to bridge the gap between scans and take into account any potential beam motion that may have occurred during these periods. Such a monitor is provided by following the quantity

$$
A_{0}=\left|\frac{A_{2}}{A_{3}-A_{2}}\right|
$$


where $A_{i}$ is the experimental asymmetry observed in channel $i$. This number is related to the zero asymmetry point of the Compton scattering spectrum, and is thus an independent monitor of the detector position. $A_{0}$ has the additional advantage of being independent of channel gain. During 1992, an additional monitor of beam motion was provided by the ratio of signal heights between an inner and outer channel. This type of monitor was not viable in 1993 because there was significant degradation of channel gain, presumably arising from polymerized gas being deposited on the reflective aluminum surfaces of the detector. However, the signal height ratio method can be used on time scales over which gain degradation is small, on the order of one week.

Figure 3-10 shows the time history of $A_{0}$ for the 1993 run. The jump in $A_{0}$ around day 190 is consistent with the 300 micron difference noted between the May 2 nd and July 15 th edge scans. The jump near day 215 corresponds to the removal of the lead beamstrahlung shield (see the next section) from the detector enclosure and is not due to beam motion. This is confirmed by observing the signal height in channel 7 relative to channel 4 during the short time period around this day.

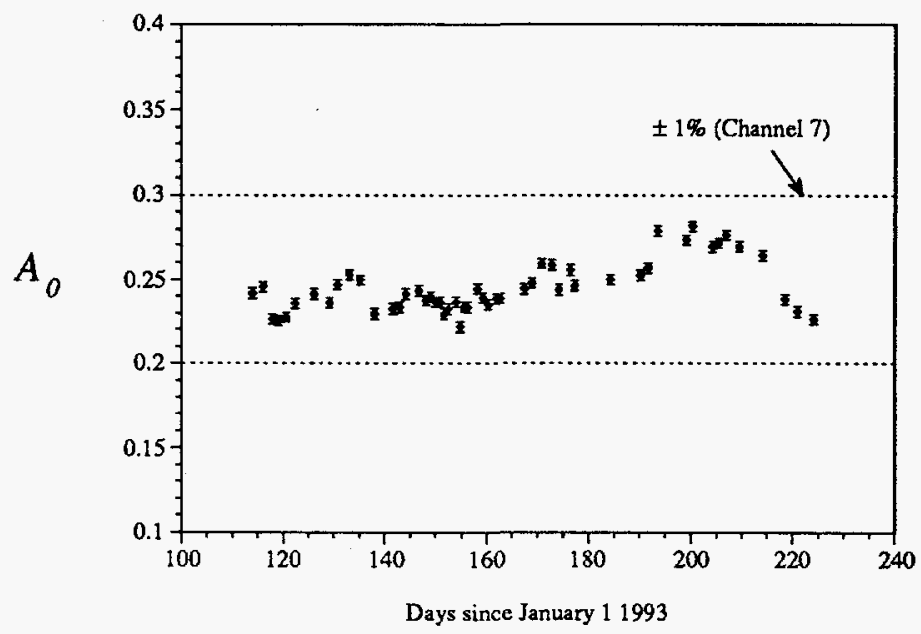

Figure 3-10: Time history of the location of the Compton zero-asymmetry point. 
Figure 3-10 shows that the beam is position is quite stable between edge scans. The combination of data from edge scans and the $A_{0}$ monitor were used to set three calibration periods, and three sets of analyzing powers were calculated based on the determined positions during these periods. The results are presented in table 3-2.

\begin{tabular}{|c|c|c|c|}
\hline Period & Calibrated Position & $a_{6}$ & $a_{7}$ \\
\hline \hline $4 / 24-7 / 10$ & $0.84 \pm 0.025$ & 0.6151 & 0.7020 \\
\hline $7 / 10-8 / 4$ & $0.87 \pm 0.025$ & 0.6118 & 0.7007 \\
\hline $8 / 4-9 / 1$ & $0.88 \pm 0.025$ & 0.6107 & 0.7003 \\
\hline
\end{tabular}

Table 3-2: Nominal Positions and Analyzing Powers by Period

\subsubsection{The Beamstrahlung Shield}

For the majority of the 1993 run, a 0.1 inch thick lead shield sat between the innermost Cherenkov detector channel (channel 1) and the aluminum gas containment cannister of the detector. This lead was inserted in order to absorb soft radiation from the beamstrahlung monitor, which is located directly across the beam pipe from the Compton detectors.

During the run, it was discovered that the ratio of the asymmetry measured in a given channel with the lead radiator in place to that measured with no radiator was slightly higher than expected. More detailed EGS simulations indicated that the presence of the beamstrahlung shielding was very likely contaminating the inner detector channels with low asymmetry showering, because the shielding itself was located very near the minimum of the Compton asymmetry curve. The extent of the contamination could not be precisely modeled due to the strong dependence on the shield position and angle, which were not well known. However, the simulations predicted negligible effects on the asymmetries measured in the outer channels.

The beamstrahlung shield was removed on July 15, 1993. Comparison of data taken after the removal versus data taken before the removal agrees with the EGS Monte Carlo predictions and confirms the hypothesis that the outer channels were unaffected by lowasymmetry contamination. An upper limit of $0.2 \%$ is placed on the uncertainty in the outer 
channel analyzing powers arising from this effect. However, because such contamination has a larger effect in the inner channels, there is a more significant uncertainty on the overall shape of the Compton spectrum.

\subsubsection{Detector Response Function}

For an ideal detector, the response function $\rho$ for a given channel is equal to a step function over the width of the channel in real space, $s$. In reality, the response function is a complicated function of the overall detector geometry, which controls the extent to which signal is shared between channels. The lead pre-radiator is the major contributor to this effect, but additional showering in the beam pipe, channel walls, etc., all serve to smear the response function beyond the channel width. These effects are modelled by an EGS4 Monte Carlo of the detector geometry. A detailed account of these calculations are found in reference [39]. Figure 3-11 shows the EGS4 generated response function for channel 7. The spikes represent signal enhancement in from showering in the aluminum channel walls, and the tails show how there is some signal cross-talk between channels.

It should be noted, however, that the total correction introduced by the Monte Carlo response function is only on the order of $1 \%$ in the outer channels, as shown in table 3-3.

Verification of the validity of the EGS modelling comes from two sources. First, the agreement of the predicted and measured tails in an edge scan, described in section 3.4.1 and shown in figure 3-9, gives qualitative support for the EGS model. Additionally, the comparison of $3 / 10$ " pre-radiator data with $1 / 10$ " pre-radiator data (table 3-4) gives more quantitative verification.

The corrections given by the response function model, which is completely dominated by the effect of the pre-radiator, are verified by the data at the level of $0.5 \%$. We further quantify this uncertainty by comparing the measured Compton asymmetry spectrum with the response function prediction, as discussed in the following section. 

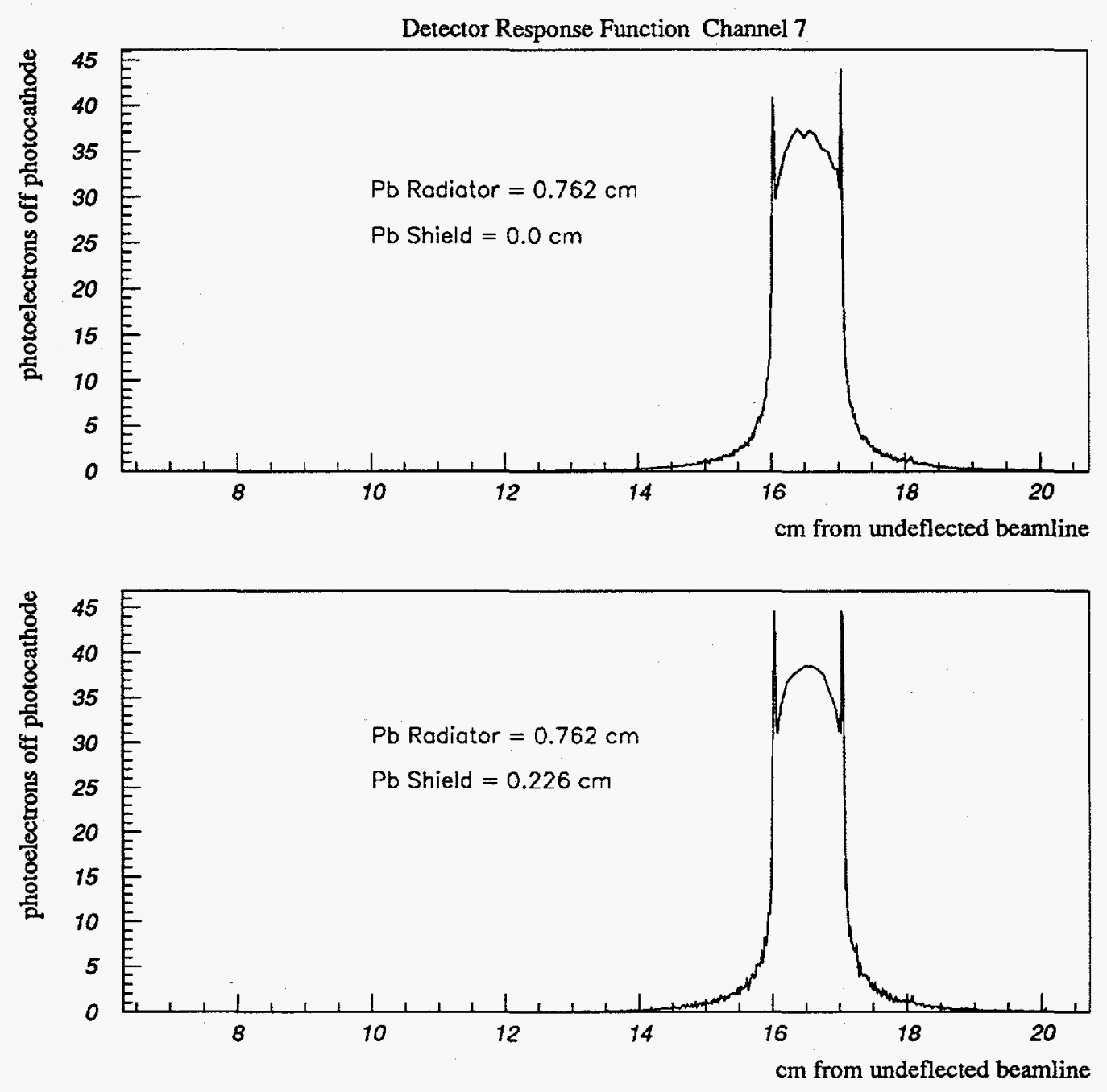

Figure 3-11: Cherenkov detector response function for channel 7. The first graph does not include the beamstrahlung shield, the second does.

\begin{tabular}{|c|c|c|c|}
\hline Channel & Ideal $a$ & Corrected $a$ & \% difference \\
\hline \hline 6 & 0.6179 & 0.6107 & 1.17 \\
\hline 7 & 0.7120 & 0.7003 & 1.64 \\
\hline
\end{tabular}

Table 3-3: Ideal and Corrected Analyzing Powers 


\begin{tabular}{|c|c|c|}
\hline Channel & $\mathrm{A}^{1 / 10} / \mathrm{A}^{3 / 10}($ Data) & $\mathrm{A}^{1 / 10} / \mathrm{A}^{3 / 10}$ (Model) \\
\hline \hline 6 & $1.008 \pm 0.0047$ & 1.007 \\
\hline 7 & $1.008 \pm 0.0045$ & 1.009 \\
\hline
\end{tabular}

Table 3-4: Thick and Thin Pre-radiator Comparison

\subsubsection{Interchannel Consistency}

To first order, the measured Compton asymmetry spectrum is a function of the detector position, and the dipole field-strength of the analyzing bend magnet. The effects of the detector response function are second-order corrections to the spectrum.

In order to compare the measured spectrum with the theoretical spectrum, we restrict ourselves to data taken after the beamstrahlung shield was removed which minimizes the Monte Carlo uncertainties on the inner channel response functions. We measured the average asymmetry in all seven channels, for all data, including runs where the laser polarization was not maximized in order to increase the statistical power. We then formed a $\chi^{2}$ function with an asymmetry curve that was a function of bend strength and polarization-product (the normalizing factor). We fit for the values of bend-strength and polarization product by minimizing this $\chi^{2}$, defined for various detector positions. The results are shown in table 3-5.

\begin{tabular}{|c|c|c|c|c|}
\hline $\begin{array}{c}\text { Fixed Edge } \\
\text { Position } \\
(\mathrm{cm})\end{array}$ & $\begin{array}{c}\text { Fit value of } \\
P_{e} \cdot P_{\gamma} \\
(\%)\end{array}$ & $\begin{array}{c}\text { Fit value of } \\
\text { bend-strength } \\
(\mathrm{MeV} / \mathrm{c})\end{array}$ & $\chi_{\text {min }}^{2}$ & Comments \\
\hline \hline 0.88 & 59.01 & 833.2 & 844.1 & bend fixed at nominal \\
\hline 0.88 & 59.18 & 825.2 & 329.7 & best-fit bend strength \\
\hline 0.85 & 58.99 & 820.2 & 334.6 & edge move by 10 \\
\hline
\end{tabular}

Table 3-5: Results of best fits to the Cherenkov spectrum.

There are several interesting observations to be made from these results. Allowing the bend-strength to be a free parameter decreases the $\chi^{2}$, but only changes the polarization by $0.3 \%$ (relative). The best-fit bend-strength is within $1 \%$ of the nominal value. Additionally, 


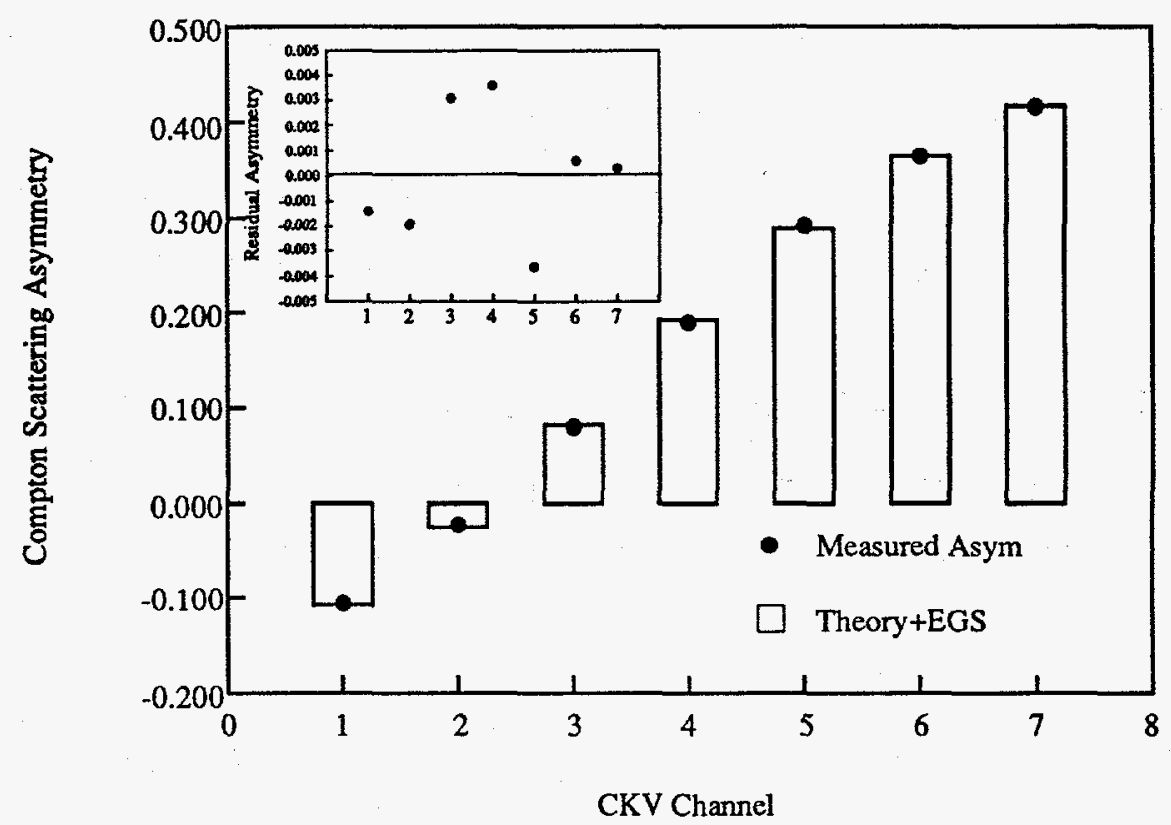

Figure 3-12: Comparison of the measured Compton asymmetry and the theoretical asymmetry, including the EGS detector response function. The residuals are shown in the inset.

moving the edge by 300 microns (about one standard deviation) has almost no effect on the $\chi^{2}$, and also only changes the polarization by $0.3 \%$. This is a good confirmation that the polarization is well understood within the systematic error of the detector calibration.

Figure 3-12 shows the data and the calculated asymmetry curve for the detector position determined by edge scan and the best-fit bend strength. The data is shown as dots, and the calculated values as vertical bars. The differences are shown in the inset residual plot. Note that the residuals are very small in the outer channels, but are more significant in the inner channels, particularly if considered as a percentage of the asymmetry measured in that channel.

The residuals shown in figure 3-12 are much larger than the statistical uncertainty on the channel measurements, and indicate additional small systematic effects that are not understood. We take the RMS residual of \pm 0.0026 to be a reasonable estimate of unknown channel-to-channel modeling uncertainties. This translates into interchannel consistency systematic errors of $0.71 \%$ and $0.62 \%$ in channels 6 and 7 respectively. 


\subsection{Target Polarization}

The largest systematic error affecting the polarimeter is the determination of the circular polarization of the target laser at the Compton interaction point, $P_{\gamma}$. The problem is that the CIP is inside the SLC vacuum and analysis optics cannot be placed there. $P_{\gamma}$ is measured at two other points: on the optics bench, after the beam has been circularly polarized, and in the analysis box, after the beam has exited the SLC vacuum. However, phase shifts induced by the laser transport optics prevent either of the monitors from directly measuring $P_{\gamma}$ A complete description of the 1993 Compton target polarization determination is found in Reference [53], the highlights of which are presented in this section.

\subsubsection{Optics}

At this point, a brief clarification of some classical optics is called for. A monochromatic, coherent packet of arbitrarily polarized (also called elliptically polarized) photons can be described by the superposition of linear polarizations about orthogonal axes:

$$
\vec{E}(t)=\left[\begin{array}{l}
E_{x} \\
E_{y}
\end{array}\right]=\left[\begin{array}{cc}
A_{x} e^{i \omega t} & e^{i \varphi_{x} t} \\
A_{y} e^{i \omega t} & e^{i \varphi_{y} t}
\end{array}\right]
$$

where $E$ is the electric field, $t$ is time, $\omega$ is the classical frequency of the light, and $\varphi_{x, y}$ are the absolute phases of the $\mathrm{x}$ and $\mathrm{y}$ components. The light is considered linearly polarized when the phase difference between the $\mathrm{x}$ and $\mathrm{y}$ components of the electric field vector, $\Delta \varphi$ $\left(=\varphi_{x}-\varphi_{y}\right)$, is zero. The light is considered circularly polarized if $\Delta \varphi=\pi / 2$ and the magnitude of each component is equal, that is, $A_{x}=A_{y}$. This state has a physical interpretation in terms of photons: it is the one in which all photons in the bunch are in the same helicity eigenstate. The circular polarization (i.e. the degree to which the light is in a helicity eigenstate) for the arbitrary light described in equation (3.21) is given by

$$
P_{\gamma}=2 \cdot \frac{\left(\operatorname{Re}\left\{E_{x}\right\} \operatorname{Im}\left\{E_{y}\right\}-\operatorname{Im}\left\{E_{x}\right\} \operatorname{Re}\left\{E_{y}\right\}\right)}{\left|E_{x}\right|^{2}+\left|E_{y}\right|^{2}}
$$


It will be shown that the combination of two Pockels cells is capable of producing arbitrary phase shifts and rotations in the polarization of the beam, that is, light of arbitrary helicity. Consider the optics arrangement shown in figure 3-13.

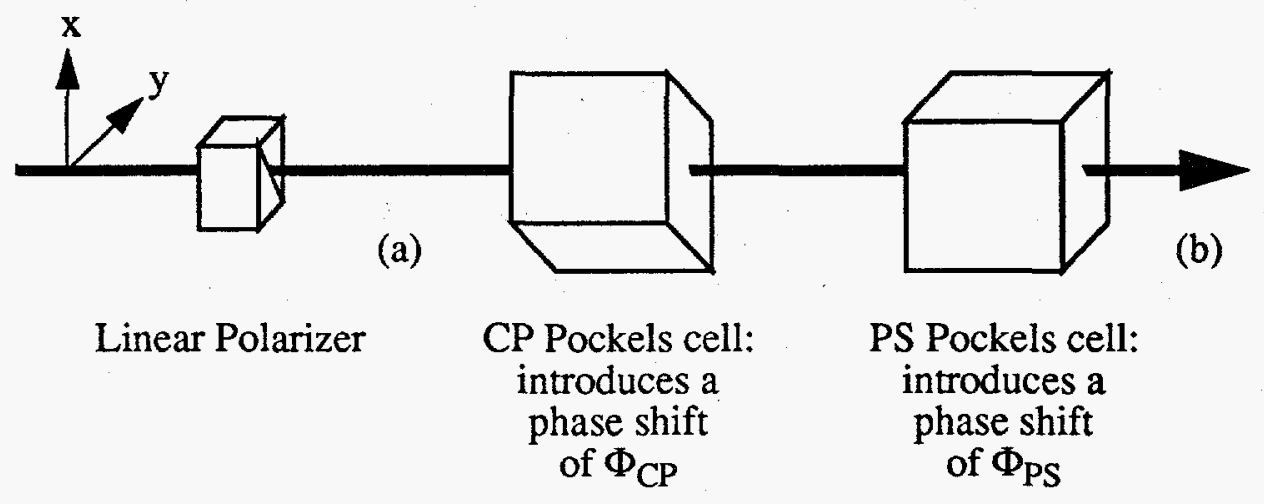

Figure 3-13: Introduction of an arbitrary phase shift.

Each optical element is mathematically represented by a $2 \times 2$ matrix which operates on the beam vector $\vec{E}$. If we define the $\mathrm{x}$-axis along the axis of the linear polarizer, the transfer matrix for the linear polarizer, $L P$ is given by

$$
L P=\left[\begin{array}{ll}
1 & 0 \\
0 & 0
\end{array}\right]
$$

Assuming that the time-dependent part, $e^{i \omega t,}$ is implicit in any beam vector, the beam vector at point (a) can be written generally as

$$
\vec{E}_{(a)}=C\left[\begin{array}{l}
1 \\
0
\end{array}\right]
$$


The CP Pockels cell has its principle axes aligned $45^{\circ}$ with respect to the linear polarizer axis (the $\mathrm{x}$-axis). Therefore its effect on the beam vector, represented by the matrix $C P$, is given by

$$
C P=\left[\begin{array}{cc}
\cos \frac{\pi}{4} & -\sin \frac{\pi}{4} \\
\sin \frac{\pi}{4} & \cos \frac{\pi}{4}
\end{array}\right]\left[\begin{array}{cc}
1 & 0 \\
0 & e^{i \Phi_{C P}}
\end{array}\right]\left[\begin{array}{cc}
\cos \frac{\pi}{4} & \sin \frac{\pi}{4} \\
-\sin \frac{\pi}{4} & \cos \frac{\pi}{4}
\end{array}\right]=\frac{\sqrt{2}}{2}\left[\begin{array}{cc}
1+e^{i \Phi_{C P}} & 1-e^{i \Phi_{C P}} \\
1-e^{i \Phi_{C P}} & 1+e^{i \Phi_{C P}}
\end{array}\right]
$$

where the rotation matrices are shown explicitly. The PS Pockels cell has its principle axes aligned with the $\mathrm{x}-\mathrm{y}$ axes, so its transformation matrix, $P S$, is simply given by

$$
P S=\left[\begin{array}{cc}
1 & 0 \\
0 & e^{i \Phi_{P S}}
\end{array}\right] .
$$

The beam vector at point $(b)$ is therefore

$$
\vec{E}_{(b)}=(P S)(C P) \vec{E}_{(a)}=C^{\prime}\left[\begin{array}{c}
\cos \left(\Phi_{C P} / 2\right) \\
i e^{i \Phi_{P S}} \sin \left(\Phi_{C P} / 2\right)
\end{array}\right] .
$$

Thus the CP Pockels cell controls the relative amplitude of the $x-y$ components, and the PS Pockels cell controls the relative phase between the components $\left(\Delta \varphi=\Phi_{\mathrm{PS}}\right)$. The circular polarization of this light is calculated through equation (3.22) to be

$$
P_{\gamma}=\sin \Phi_{C P} \cos \Phi_{P S}
$$

\subsubsection{Pre-AUTOPOCKSCAN Analysis}

At the beginning of the 1993 run, we took the following approach: An intrusive measurement was made at the Compton IP by breaking the SLC vacuum. $P_{\gamma}$ was found to be $(98 \pm 1) \%$. It was then assumed that the transport line phase shifts were constant in time. Under this assumption, the analysis box optics could measure the stability of $P_{\gamma}$ although not $P_{\gamma}$ itself. This period of time during which this assumption was the basis of the laser light analysis is now called the pre-AUTOPOCKSCAN era, which lasted through April 27, 
1993. All running beyond this date is referred to as the AUTOPOCKSCAN era, which is described in the next section.

As it turns out, the phase shifts induced by the laser transport system were not constant in time. This fact is illustrated in figure 3-14, which is a time history of the laser polarization measured in the analysis box, $P_{\gamma}{ }^{A B}$, for the first forty days of the 1993 run. This era is divided into seven epochs, based on the stability of the asymmetry measurements. The jumps in $P_{\gamma}^{A B}$ during a given epoch do not correspond to any changes in the measured Compton asymmetry and are therefore due to changes in the transport line phase shifts rather than real changes in $P_{\gamma}$. The larger jumps were probably a result of optical damage (or the replacement of damaged optical components) caused by laser "hot-spots" (points of unusually high intensity within the beam profile). These hot-spots were in turn caused by a failing Q-switch mechanism in the laser head, which was eventually replaced. The small jumps (between epochs II and III for instance) are probably related to Pockels cell alignment, and may also be related to environmental factors such as temperature.

During the first three epochs of this era, there was only one Pockels cell (the CP cell) installed on the laser bench. However, between epochs II and III the principal axes of this cell were rotated by $45^{\circ}$. This means that the combination of the manual voltage scans taken in each of these two eras actually serves to map the phase shifts along two orthogonal axes, and thus an overall $P_{\gamma}$ for the two eras is determined. (The method by which $P_{\gamma}$ is determined through Pockels cell voltage scans is described in the next section.)

Starting with epoch IV, the PS Pockels cell was installed on the CLS laser bench. Manual voltage scans of both cells in epochs IV, V, and VI, allow determinations of $P_{\boldsymbol{\gamma}}$ for each of those epochs. Automated scans were implemented at the end of epoch VII, and because there were no optics or transport line changes between epoch VII and the start of the scanning era, the determinations made during the first auto-scans are projected backward in time to include epoch VII. A summary of the $P_{\gamma}$ determinations and methods used for this era is given in table 3-6.

The systematic error on all $P_{\gamma}$ measurements in the pre-AUTOPOCKSCAN era is estimated to be $2.1 \%$. Approximately $27 \%$ of all $Z$ data was taken during this era. 


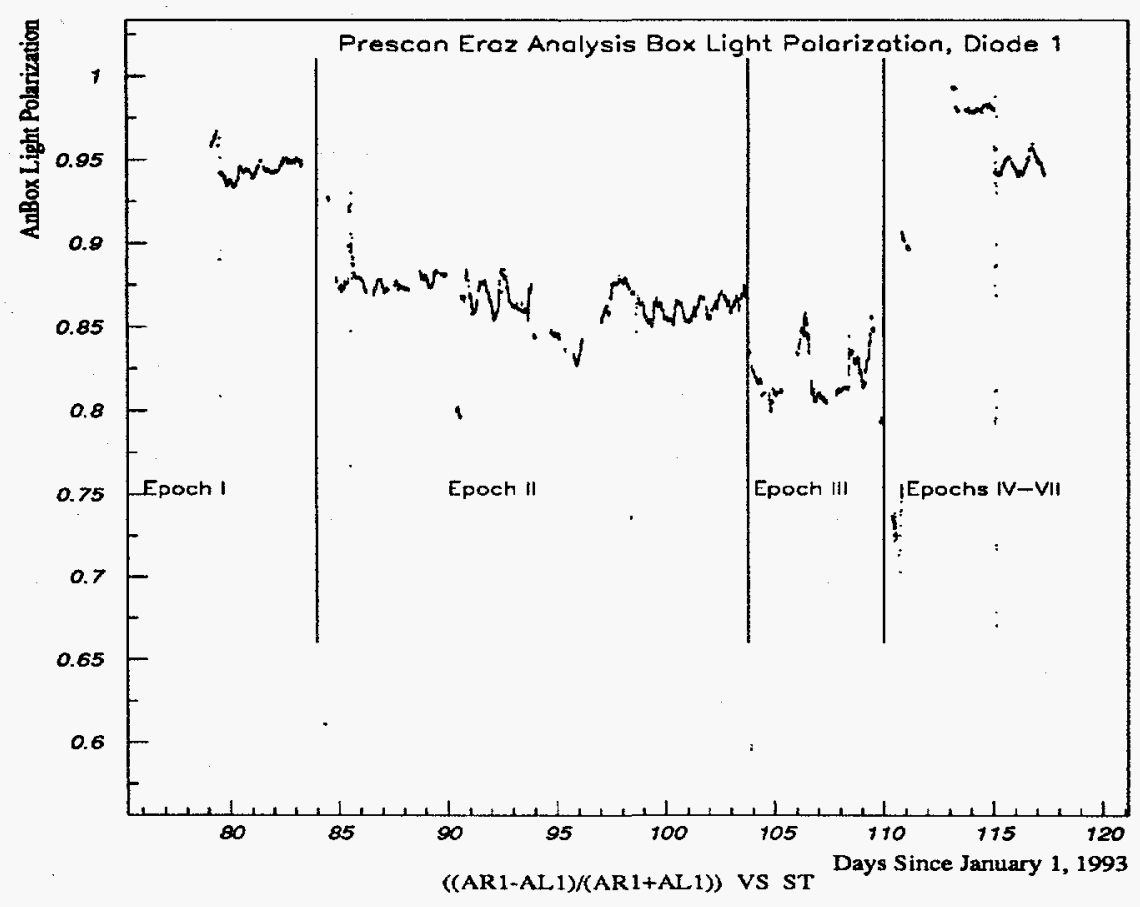

Figure 3-14: $P_{\gamma}^{A B}$ in the Pre-AUTOPOCKSCAN era.

\begin{tabular}{|c|c|c|}
\hline Epoch & $P_{\gamma}$ & Method by which $P_{\gamma}$ is determined \\
\hline \hline I & 0.98 & Intrusive direct measurement at CIP \\
\hline II & 0.96 & Manual scan of CP phase \\
\hline III & 0.96 & Manual scan of PS phase \\
\hline IV & 0.93 & Manual scan of both cells \\
\hline V & 0.99 & Manual scan of both cells \\
\hline VI & 0.97 & Manual scan of both cells \\
\hline VII & 0.99 & Automated scanning implemented \\
\hline
\end{tabular}

Table 3-6: $P_{\gamma}$ measurements during the pre-AUTOPOCKSCAN era. 


\subsubsection{AUTOPOCKSCAN Analysis}

Once the phase shift instability of the transport optics became apparent, a new strategy for monitoring said phase shifts and measuring $P_{\gamma}$ was implemented. This strategy involves two different types of automated scanning: one in which the Pockels cell voltages are scanned versus the measured Compton asymmetry (EPOL scan), and the other in which the voltages are scanned versus the analysis box photodiode signals (LP scan). EPOL scans allow us to determine how far from the maximum achievable polarization we are at a given voltage setting. LP scans allow us to determined the value of the maximum achievable polarization. Only the combination of both types of scans allows an absolute measurement of $P_{\gamma}$ for a given time period.

Nominal voltages on the Pockels cells are defined by those voltages which maximized the Compton asymmetry during a previous scan. In both types of scans, the voltages on each Pockels cell are scanned independently, that is, one cell is held at nominal voltage while the other cell is scanned ( $V_{\text {offset }}= \pm 800, \pm 600, \pm 400$, and \pm 200 volts) beyond its nominal setting. When the $\mathrm{CP}$ cell was scanned, it voltage was flipped pseudo-randomly during each run between $\pm\left(\mathrm{V}_{\text {nominal }}^{\mathrm{CP}}+\mathrm{V}_{\text {offset }}\right)$, while the PS cell was held at its nominal voltage. When the PS cell was scanned, the CP cell alternated between $\pm\left(\mathrm{V}^{\mathrm{CP}}{ }_{\text {nominal }}\right)$ while the PS cell was set to $\left(\mathrm{V}^{\mathrm{PS}}{ }_{\text {nominal }}+\mathrm{V}_{\text {offset }}\right)$.

EPOL scans take place continually. The cell voltages are set, and a normal 20,000 event Compton run is taken. A nominal run (i.e. both cells set to nominal voltages) is taken in between each pair of scan points. That means ( 2 cells) $x(4$ voltage offsets) $x$ ( 3 voltage settings per offset $\left[ \pm V_{\text {offset }}\right.$ and $\left.\left.V_{\text {nominal }}\right]\right)=24$ runs per scan, which takes slightly more than one hour to complete. During this time, eight nominal runs are taken, approximately one nominal run every eight minutes.

At the end of each EPOL scan, an LP scan is scheduled. The LP scan consists of eighty points, forty scanning the $\mathrm{CP}$ voltages and 40 scanning the PS voltages. Each point consists of 100 beam crossings, therefore the total length of the scan is 8000 beam crossings, or just over one minute. 
A combination of off-line best-fits to the two types of scans determines $P_{\gamma}$ The photodiode signals measured during an LP scan, $P D_{ \pm}{ }^{i}$, during which the Pockels cell voltages are set to $V_{C P}^{i}$ and $V_{P S}^{i}$, are fit to the following function

$$
P D_{ \pm}^{i}=G_{ \pm}\left(1+U \pm \sin \left[\left(\frac{V_{C P}^{i}-\Phi_{C P}^{A B}}{V_{C P}^{\lambda / 4}}\right) \frac{\pi}{2}\right] \cos \left[\left(\frac{V_{P S}^{i}-\Phi_{P S}^{A B}}{V_{P S}^{\lambda / 4}}\right) \frac{\pi}{2}\right]\right)
$$

Here, the " + " ("-") photodiode is defined to be the one which sees a large signal with a positive (negative) voltage on the $\mathrm{CP}$ Pockels cell. $G$ is the gain of the photodiode, $U$ is the fraction of unpolarized light in the laser beam, and the $\Phi^{\mathrm{AB}}$ are the uncompensated phase shifts along the $C P$ and $P S$ axes observed in the analysis box, in units of volts.

There are two phases of LP scan analysis. During the first phase, the unpolarized fraction $U$ is set to zero, and $\Phi^{\mathrm{AB}}$ and $V^{\lambda / 4}$ are free parameters of the fit. During the second phase, all variables except $U$ are fixed, and $U$ is determined by fitting only the data very near the minimum of the photodiode extinction curve. This is done because all information on the unpolarized fraction, which is related to the deviation of the minimum from zero, comes from points in this neighborhood, and because the fit convergence improves greatly with this restriction.

EPOL scans are fit to the following function

$$
A_{6}^{m, i}=\left(P_{e} P_{\gamma}\right) \sin \left[\left(\frac{V_{C P}^{i}-\Phi_{C P}^{C I P}}{V_{C P}^{\lambda / 4}}\right) \frac{\pi}{2}\right] \cos \left[\left(\frac{V_{P S}^{i}-\Phi_{P S}^{C I P}}{V_{P S}^{\lambda / 4}}\right) \frac{\pi}{2}\right]
$$

where the measured asymmetry $A^{m}$ is as defined in equation (3.13) and the polarization product is a single parameter. For this fit the quarter-wave voltages are fixed at the values determined by the most recent $L P$ scan. The $\Phi^{C I P}$ represent the uncompensated phase shifts at the Compton interaction point. Note that as the $\Phi^{C I P}$ become larger, the uncertainties on the $\Phi^{C I P}$ have a greater effect on the determination of $P_{\gamma}$. For this reason, the nominal voltages were reset by polarimeter experts whenever either $\Phi^{C I P}>200 \mathrm{~V}$. Ideally, we would set $V_{C P}^{\text {nominal }}=V_{C P}^{\lambda / 4}+\Phi_{C P}^{C I P}$ and $V_{P S}^{\text {nominal }}=\Phi_{P S}^{C I P}$ at the end of each EPOL scan, but this 
is not possible without on-line fitting and would be dangerous because of the possibility of a bad fit setting the voltages far away from the true nominal.

The laser polarization from a set of LP and EPOL scans is now given by

$$
P_{\gamma}=(1-U) \sin \left[\left(\frac{V_{C P}^{\text {nominal }}-\Phi_{C P}^{C I P}}{V_{C P}^{\lambda / 4}}\right) \frac{\pi}{2}\right] \cos \left[\left(\frac{V_{P S}^{\text {nominal }}-\Phi_{P S}^{C I P}}{V_{P S}^{\lambda / 4}}\right) \frac{\pi}{2}\right]
$$

where the quarter-wave voltages are determined by the first phase of the LP fits, $U$ is determined by the second phase of the LP fits, and the $\Phi^{C I P}$ are determined by the EPOL fits.

Figure 3-15 shows the time history of all $P_{\gamma}$ measurements in the AUTOPOCKSCAN era. Approximately $73 \%$ of the $Z$ data was taken during this time period. The systematic uncertainty on $P_{\gamma}$ during this era is $0.6 \%$.

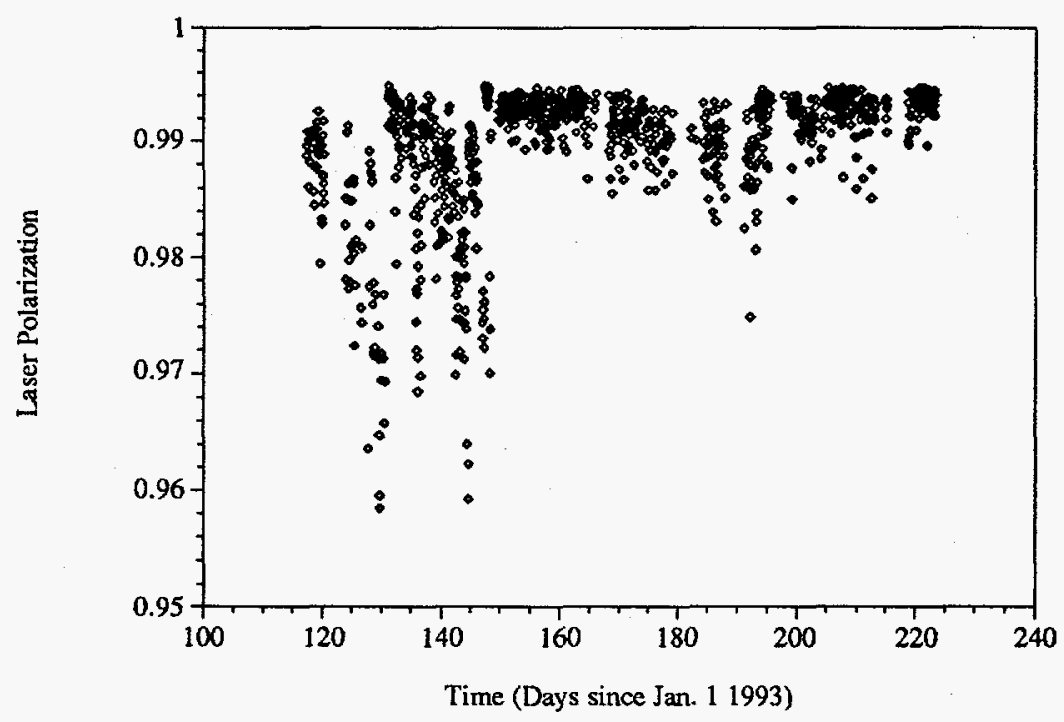

Figure 3-15: Time history of $P_{\gamma}$ during the AUTOPOCKSCAN era.

\subsection{Absolute Sign Determination}

The determination of the absolute sign of the electron polarization is of course essential to the $A_{L R}$ analysis, since $A_{L R}$ requires counting the number of left and right-handed $Z s$. Recall 
from equation (3.13) that the measured asymmetry is equal to the product of the laser polarization, the electron polarization, and the analyzing power Given these inputs, we have sufficient information to determine the sign of the electron polarization. We have discussed the asymmetry measurement in section 3.3 . The analyzing power is calculated from the theoretical Compton asymmetry, this sign of which is discussed below. The absolute target helicity is monitored on a pulse-by-pulse basis, and is also described in this section.

\subsubsection{Sign of the Compton Scattering Asymmetry}

In 1992, there was a period of excitement due to the fact that the measured value of $A_{L R}$ was negative, contrary to the expectations of the Standard Model. This discrepancy was traced to a theoretical error on the sign of the Compton scattering asymmetry. Most references deftly avoid mentioning the sign of the asymmetry, and of those that do make a claim, some are incorrect. The correct result is that fully backward Compton scattering (corresponding to the Compton kinematic endpoint) has a larger cross section when the electron and photon helicities are parallel [52].

This result can be understood through the following argument: Consider the highenergy limit of fully-backward Compton scattering, when both the electron and photon exist in helicity eigenstates. Given that the longitudinal component of angular momentum, $j_{z}$, must be conserved, it follows that the spin direction of the electron and photon in the final state must be the same as in the initial state. This means that the helicities of the photon and electron will change sign. If angular momentum is also conserved at each vertex of the exchange diagrams (figure 3-1), it also follows that the $j_{2}=3 / 2$ initial state must be preferentially occur through the $u$-channel exchange, while the $j_{2}=1 / 2$ initial state must preferentially occur through the $s$-channel diagram. All that remains to be seen is that the contribution from the $u$-channel, whose propagator (in the denominator) is proportional to the electron mass-squared, is greater than the $s$-channel, whose propagator is proportional to the center-of-mass energy-squared.

To summarize, for fully backward scattering, the $u$-channel dominates the $s$-channel, and only the $j_{z}=3 / 2$ state contributes to the $u$-channel. Therefore, the state in which the inci- 
dent photon and electron helicities are parallel (corresponding to the $j_{z}=3 / 2$ state) has the larger cross section at the Compton kinematic endpoint.

\subsubsection{Helicity of the Target Photons}

A quarter-wave plate is an optical element which, for a given wavelength, imparts $90^{\circ}$ of phase advance for fields projected along the fast axis relative to those along the slow axis. In order to measure the helicity of polarized light, one requires a quarter-wave plate with an identified slow axis. In 1992, the PLS and CLS quarter-wave plates were calibrated by several optical techniques, which are discussed in detail in Reference [54], and are briefly described below.

\section{Fresnel Total Internal Reflection}

This method relies on the fact that after a total internal reflection, the components of linear polarization parallel and perpendicular to the plane of reflection undergo different phase shifts. This is considered the primary method of quarter-wave plate calibration because the results are derived from first-principles [55]. Subsequent techniques rely on commercial optics equipment and standards.

\section{Circular Dichroism}

This technique relies on the fact that certain materials in solution preferentially transmit one handedness of polarized light. We used a standard reference solution of cyano-cobalamin (vitamin B-12) and a commercial circular-dichroism spectropolarimeter at a Stanford biochemistry laboratory, and tested four quarter-wave plates.

\section{Berek Compensator}

A Berek Compensator is an adjustable retarder plate consisting of a single birefringent crystal $\left(\mathrm{MgF}_{2}\right)$ whose slow axis is oriented perpendicular to the plate surface. At the proper setting, the Berek Compensator acts as a quarter-wave plate with an identified slow axis, and such a quarter-wave plate can be used to find the slow axis of a second quarter-wave plate. Two of the CLS quarter-wave plates were tested in a commercial laboratory using this technique. 


\section{Liquid Crystal Polarizer}

Each of the above slow-axis determinations agreed with the other, as well as with the manufacturer's marking of the slow axis, on those plates that had unambiguous markings. From this extensive research came a relatively simple tool for photon helicity determination: the liquid-crystal polarizer, or LCP technique.

A liquid crystal polarizer is a commercial device that transmits light of right-handed helicity and reflects light of left-handed helicity with an extinction ratio of several hundred to one. In 1992, two LCPs purchased by SLAC were used to test quarter-wave plates, and agreed with the previous calibrations. In 1993 a LCP optimized to the Compton laser wavelength was installed on the optics bench to provide a continuous monitor of the light helicity. Because all light transport between the laser bench and the Compton interaction point involves mirror pairs mounted in mirror boxes (discussed in section 2.3.1), it is guaranteed that there are an even number of reflections between these points. Since each single reflection changes the handedness of the polarization, it therefore follows that the handedness at the CIP must be the same as that measured on the bench. A study of the LCP photodiode signals for the entire run shows that the CLS 01 state corresponded to the righthanded photon state, and that the meaning of this assignment never changed.

Given this information, it is possible to infer the electron beam helicity assignments for the entire run, given the sign of the measured Compton asymmetry at the kinematic endpoint (measured by $A_{7}^{m}$ ). The sign of $A^{m} 7$ changed only once during the run, on June 10 , 1993. This corresponded to the time at which the current on the LTR solenoid was reversed, changing the orientation of spins in the damping ring, and reversing the meaning of the CLS helicity assignments.

\begin{tabular}{|c|c|c|c|}
\hline Era & $\begin{array}{c}\text { CLS 01 state corresponds } \\
\text { to this helicity: }\end{array}$ & $\begin{array}{c}\text { sign of } \\
A^{e}\end{array}$ & $\begin{array}{c}\text { PLS 01state corresponds to } \\
\text { this helicity: }\end{array}$ \\
\hline \hline $4 / 10-6 / 10$ & $\mathrm{R}$ & negative & $\mathrm{R}$ \\
\hline $6 / 10-9 / 1$ & $\mathrm{R}$ & positive & $\mathrm{L}$ \\
\hline
\end{tabular}

Table 3-7: Inferred Electron Helicity during 1993 run 
Changing the sign of the LTR solenoidal field is one of many methods by which systematic errors affecting $A_{L R}$ may be reduced. Future experiments may benefit by more frequent reversals of this type. The LCP provides a simple and safe monitor of the PLS and CLS helicity assignments, which will change when such reversals are made.

\subsection{Polarimeter Data Selection}

The operation of the polarimeter has been described in chapter 2 . The important details are the following: data for each Cherenkov channel are acquired in twelve histograms, based on 3 possible electron beam states (left polarized, right polarized, and unpolarized) and 4 possible Compton target states (left polarized, right polarized, unpolarized, and off). A typical polarimeter run consists of 20,000 beam crossings, or approximately three minutes of live beam time. Runs may be cut short by loss of the electron beam or operator overrides. At the end of each run, a number of run-stable quantities are read, such as detector voltages and detector position, and are included in the data structure for the run.

The data are required to satisfy a number of run-specific and channel-specific selection criteria. Every run must pass all run-specific cuts in order to be included in the analysis. If neither channel 6 nor channel 7 passes all of the channel-specific cuts then the run is dropped from the analysis. If both channels 6 and 7 pass all of the channel-specific cuts, then the polarization for the run is the average measurement for the two channels (this has the effect of lowering the total systematic error; refer to the following section). Otherwise the measurement is reported only for the channel that passed all of the channel-specific cuts.

Runs which pass all run-specific cuts and have at least one channel that passes the channel-specific cuts form a data set from which the luminosity-weighted polarization, $\left\langle P_{e}^{C}\right\rangle$, is calculated. 


\subsubsection{Run-specific Cuts}

\section{Number of Events}

We require at least 100 events in each of the four possible helicity combinations of electrons and photons ( $\mathrm{LL}, \mathrm{RR}, \mathrm{LR}, \mathrm{RL}$ ). This cut primary assures that the calculated statistical error on the run is valid, and also removes the operator "one-shots" (test runs with the run length set to 100) at an early stage of the analysis.

\section{Nominal Runs}

Only runs during which the CLS Pockels cells are set to their nominal voltage are included in the polarimeter analysis. During the automated Pockels cell scanning era, a only one-third of the total polarimeter data were taken at nominal voltages. The systematic error on the determination of the laser polarization is smallest for nominal runs, because these runs occur at points where the derivative of the EPOL fit function [equation (3.29)] approaches zero. We could include all off-nominal runs and their corresponding $P_{\gamma}$ determinations, but there is nothing to gain by this except an inflated systematic error, since we are in no way statistically limited by the number of nominal runs (again a result of needing to know only the average polarization for the entire run).

Pockels cell nominal voltages were determined based on database entries and a careful reconstruction of Polarimeter logbook entries. The nominal voltages were checked by observing the continuity (in the mathematical sense) of the polarimeter data. Eras during which the incorrect nominal voltages were asserted would appear as gaps of no data or data with anomalously low polarization.

\section{Veto Status}

We require that the electron toroid veto was set, which is to say, we require that missing-electron pulses were not summed in the histograms of the run in question (vetoes in general were described in section 2.3.4). Because the beam helicity code for the electrons is based on the electron source, and has no information about whether the electrons were dumpered at the end of the linac, runs for which the toroid veto was not operational would 
erroneously include the missing-electron pulses in one of the four signal states. This would effectively lower the measured asymmetries and thus the extracted electron polarizations for such runs.

\section{Lead Pre-radiator Status}

We require that there be $2 / 10 ", 3 / 10 ", 1 / 3 "$, or 1 " of lead pre-radiator in front of the Cherenkov detector, as these are the thicknesses for which the Cherenkov analyzing powers have been calibrated. At this point in the analysis, the base analyzing powers are selected for the given pre-radiator configuration and calibration era.

\section{Detector Position}

We require that the detector platform be within $1.36 \mathrm{~mm}$ of its nominal position. Within this range, the base analyzing powers are corrected for any deviation from the nominal position. Any runs with the platform outside of this range are dropped.

\subsubsection{Channel-specific Cuts}

\section{Signal-plus-Background}

The average signal plus background must be greater than 35 counts. This cut eliminates runs in which the laser noise pick-up correction becomes very significant.

\section{The Spin-aligned Pedestal-subtracted Signal $\left(\mathrm{PH}^{+}\right)$}

This quantity is required to be greater than 40 and less than 440 . The linearity of both channels has not been measure with sufficient statistics below $\mathrm{PH}^{+}<40$. The uncertainty on the linearity corrections was deemed too large for $\mathrm{PH}^{+}>440$. Channel 7 asymmetries are corrected to the measured linearity curve for $40<\mathrm{PH}^{+}<440$. Channel 6 asymmetries are corrected when $150<\mathrm{PH}^{+}<440$. 


\section{Statistical Error}

The statistical error on the measurement of a given state is given by

$$
\sigma^{X Y}=\sqrt{\frac{1}{N^{X Y}}\left(\frac{\sum\left[O_{i}^{X Y}\right]^{2}}{N^{X Y}}-\left[\frac{\sum o_{i}^{X Y}}{N^{X Y}}\right]^{2}\right)+\frac{1}{N_{B}^{X}}\left(\frac{\sum\left[B_{j}^{X}\right]^{2}}{N_{B}^{X}}-\left[\frac{\sum B_{j}^{X}}{N_{B}^{X}}\right]^{2}\right)}
$$

where $O_{i}^{X Y}$ is the ith observation of signal-plus-background in the XY state, $N^{X Y}$ is the total number of signal observations in that state, $B_{j}^{X}$ is the jth observation of background in the $\mathrm{X}$ state, and $N_{B}{ }^{X}$ is the total number of background observations in the $\mathrm{X}$ state. The dominant statistical effect in the 1993 polarimeter was the intensity-jitter of the target laser. Thus the variance of the signal-plus-background is much greater than the variance of the background alone. Additionally, the background was measured much more often than the signal; on average, $N_{B}{ }^{X} \approx 20 \cdot N^{X Y}$. Therefore the statistical error on a background measurement makes a negligible contribution to the statistical error for a run.

The polarization product measured by a given channel $\mathrm{i}, P P_{i}$, is given by

$$
P P_{i}=\left(P_{\gamma} P_{e}^{C}\right)_{i}=\frac{A_{i}^{e}}{a_{i}}
$$

where the experimental asymmetry and the analyzing power were defined earlier in this chapter. It follows from equations (3.13) and (3.33) that the statistical error on the polarization product, $\sigma_{i}^{P P}$ is given by

$$
\sigma_{i}^{P P}=\frac{\sqrt{\left(1-P P_{i}\right)^{2}\left(\left[\sigma_{i}^{L L}\right]^{2}+\left[\sigma_{i}^{R R}\right]^{2}\right)+\left(1+P P_{i}\right)^{2}\left(\left[\sigma_{i}^{L R}\right]^{2}+\left[\sigma_{i}^{R L}\right]^{2}\right)}}{a_{i} \cdot\left(N_{i}^{L L}+N_{i}^{R R}+N_{i}^{L R}+N_{i}^{R L}\right)}
$$

The statistical error on the polarization product is required to be less than $3 \%$ absolute in order for the run to be included in the calculation of the luminosity-weighted polarization. Note that, as the luminosity-weighted polarization is a weighted average of over 20,000 polarimeter runs, the statistical error on an individual run is irrelevant, and the statistical error on the luminosity-weighted polarization is negligible compare to the systematic error. 


\subsubsection{The Final Compton Polarization}

If a polarimeter run has passed the data quality cuts, the polarization product is calculated from the measured Compton asymmetry, as indicated in equation (3.33). The laser polarization is then read from a database. If the polarimeter run occurs during the pre-scan era, the laser polarizations are drawn from table 3-6. If the run occurs during the scan era, the laser polarization measurements (shown in figure 3-15) are searched, and the determination made closest in time to the polarimeter run is applied to the polarization product, in order to extract the final Compton polarization, $P_{e}{ }^{C}$.

\subsection{Polarimeter Systematic Errors}

To summarize the systematic errors discussed in this chapter:

The detector calibration error reflects the uncertainty of the location of the detector relative to the Compton spectrum, and includes the error on an edge scan determination and the drift in electron beam position between edge scans. The interchannel consistency error reflects the uncertainty on the shape of the measured asymmetry, and includes contributions from the uncertainty in the analyzing bend magnet field strength and the detector response function simulation. As each channel makes and independent asymmetry measurement, this error is uncorrelated between channels. The linearity uncertainty reflects the statistical limit on the measurement of the asymmetry versus pulse-height curves. Because the channel 7 linearity curve relies on a normalization to the corrected channel 6 data, these uncertainties are partially correlated between the two channels. The inner shielding uncertainty is an upper limit on low asymmetry background contamination coming from the beamstrahlung shield. The electronic noise uncertainty is an upper limit on electronic crosstalk between channels. The laser polarization error is the weighted mean of the $0.6 \%$ error on the laser polarization during the automated Pockels cell scanning era and the $2 \%$ laser polarization determination during the pre-scan era.

Contributions to the total polarimeter systematic error are summarized in table 3-8. Note that there is indeed an advantage to the averaging of channel 6 and channel 7 polarization measurements, which brings the total systematic error down to $1.3 \%$. 


\begin{tabular}{|c|c|c|c|c|}
\hline Source & $\begin{array}{c}\text { Channel 6 } \\
\text { Error (\%) }\end{array}$ & $\begin{array}{c}\text { Channel 7 } \\
\text { Error (\%) }\end{array}$ & correlation & $\begin{array}{c}\text { Combined } \\
\text { Error (\%) }\end{array}$ \\
\hline \hline IC Consistency & 0.71 & 0.62 & 0.00 & 0.47 \\
\hline Linearity & 0.70 & 0.70 & 0.50 & 0.61 \\
\hline Calibration & 0.50 & 0.14 & 1.00 & 0.32 \\
\hline Inner Shielding & 0.20 & 0.20 & 1.00 & 0.20 \\
\hline Electronic Noise & 0.20 & 0.20 & 1.00 & 0.20 \\
\hline Laser Polarization & 1.00 & 1.00 & 1.00 & 1.00 \\
\hline \hline TOTAL: & 1.52 & 1.41 & & 1.33 \\
\hline
\end{tabular}

Table 3-8: Total Systematic Error for Individual Channels and Combined Channels

\subsection{The Luminosity-weighted Polarization}

It was shown in chapter 1 that the measurement of $A_{L R}$ depends only on the luminosityweighted polarization, $\left\langle P_{e}\right\rangle$. We estimate this value through the following relation

$$
\left\langle P_{e}^{C}\right\rangle=\frac{1}{N_{Z}} \cdot \sum_{i=1}^{N_{Z}}\left|P_{i}^{C}\right|
$$

where $N_{Z}$ is the total number of $Z$ s observed, and $P_{i}^{C}$ is the polarization measurement most closely associated in time with the $i$ th $Z$. We will discuss the selection of $Z$ s in the next chapter; taking the Zs as given, the time history of $P_{i}^{C}$ is shown in figure 3-16 (A).

The one-dimensional projection of this time history is shown in figure 3-16 (B). The luminosity-weighted Compton measured electron polarization for the 1993 run is

$$
\left\langle P_{e}^{C}\right\rangle=0.6190 \pm 0.0082
$$

where the quoted error is purely systematic. 


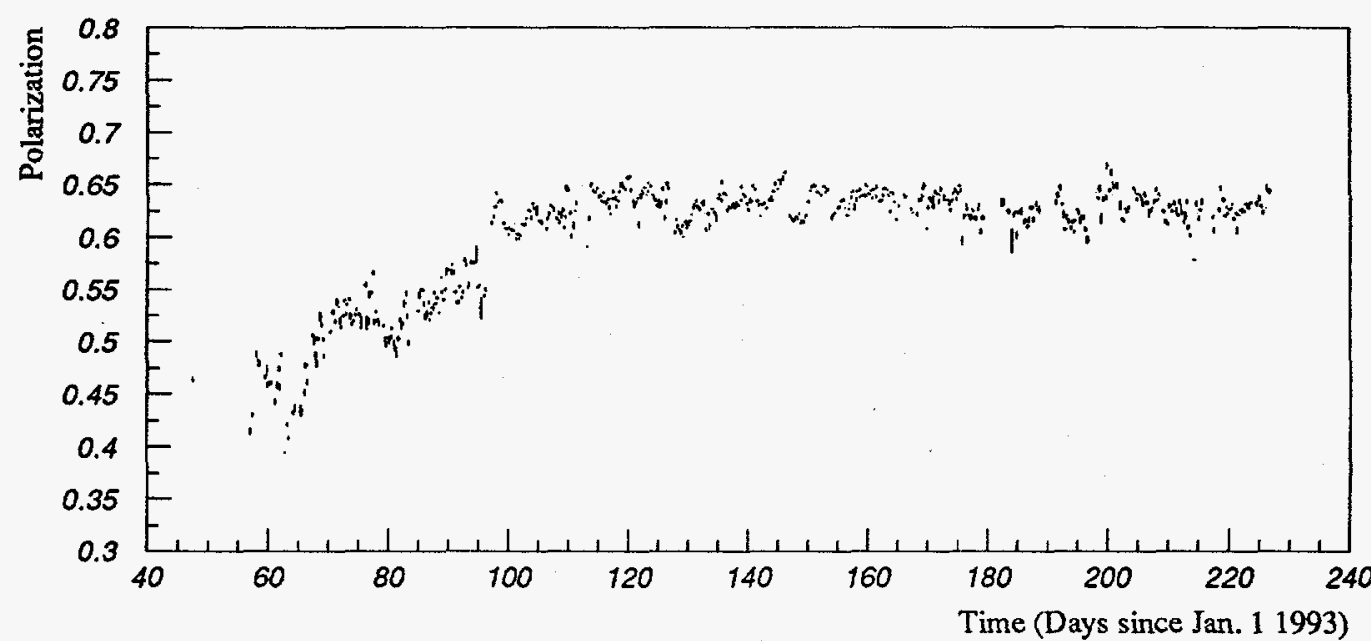

(A)

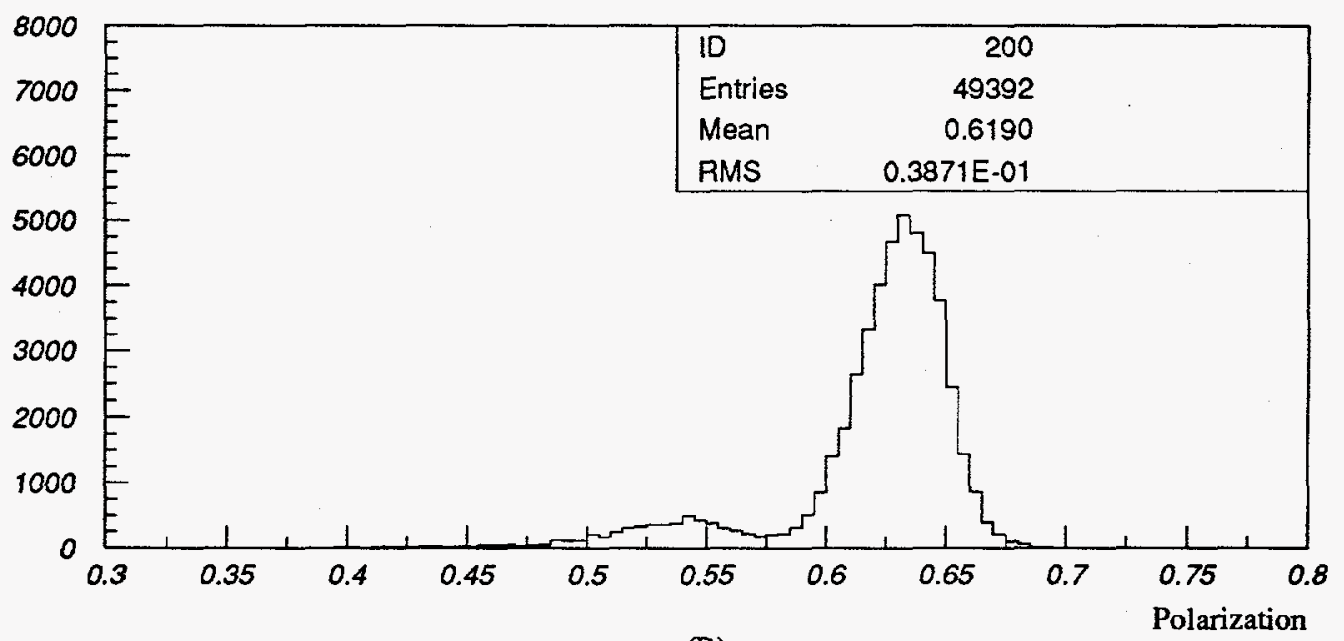

(B)

Figure 3-16: The 1993 Compton measured polarization.

(A) Time history of the polarization in 6-hour blocks.

(B) Histogram of luminosity-weighted polarization. 


\section{Chapter 4}

\section{Z Event Selection and Background Estimation}

This chapter will provide the details of the 1993 event selection strategy and background estimation. The analysis presented is unique in that it depends on calorimetric data only; no tracking is required. The calorimeter has a large acceptance (up to $\cos \theta=0.98$ ), and a large livetime, because the calorimeter triggers can be read out in less than a single SLC cycle $(8.3 \mathrm{~ms}$ ). As a result a high overall detection efficiency (approximately $94 \%$ ) is obtained.

The $A_{L R}$ measurement does not have particularly stringent event selection requirements. Because $A_{L R}$ is independent of the final state of the $Z$ decay, we can accept almost any produced $Z$ event. The exception is the electron-positron final state, also referred to as the Bhabha final state. This is the dominant background in the measurement. Other backgrounds to be considered are: beam-related background events, muon halo events, cosmicrays, and so-called "two-photon" events.

\subsection{The LAC Energy Scale}

In order to describe the event selection criteria, we must first discuss the calibration of the calorimeter energy scale. The signal size that is associated with a LAC tower is converted 
into an energy based on the minimum ionizing energy scale (or $\mu$ scale). This scale is based on the assumption that the charge deposited arises from a minimum ionizing particle that loses energy in the lead and liquid argon.

The $\mu$ scale conversion factors were determined by normalizing the minimum ionizing peak, seen in the LAC EM sections from tracks in hadronic events, to the expected value [56]. These conversion factors are $2.28 \times 10^{-3} \mathrm{GeV} / \mathrm{ADC}$ count in the EM sections and $5.99 \times 10^{-3} \mathrm{GeV} / \mathrm{ADC}$ count in the HAD sections. All energies discussed in the event selection criteria of this chapter are defined in terms of this minimum ionizing scale.

The LAC is a sampling calorimeter, which means the total energy passing through the calorimeter is not measured. Some energy is absorbed by the radiator plates themselves, and what fraction of the total energy is sampled is a function of the shower type. For instance, hadronic showers can lose energy through neutrons in hadronic showers, neutrinos in pion decay, and also through nuclear binding energy in hadronic production. Therefore the ratio of the hadronic energy scale (the $\pi$ scale) to the $\mu$ scale, $\pi / \mu$, is less than one. Similarly, in electromagnetic showers, the very soft (low momentum) shower products have a lower efficiency for charge conversion in the calorimeter. Some of this energy is not sampled, and the ratio of the electromagnetic energy scale to the minimum ionizing scale, $\mathrm{e} / \mu$, is also less than one.

Because of these different mechanisms of energy loss, the ratio of the electromagnetic to hadronic energy scales, e/ $\pi$, is not equal to one. An analysis of the 1992 data [57] measures this ratio to be approximately 1.7. As a result, leaving calorimeter energies in the $\mu$ scale means that there is a greater separation between purely electromagnetic final states $\left(\mathrm{e}^{+} \mathrm{e}^{-}\right)$and hadronic final states. This is actually an advantageous situation for reasons discussed in section 4.4.1.

\subsection{Event Triggers}

It is clearly not feasible to write out the state of the SLD detector for every beam crossing. We must have some criteria by which the large quantity of noise is ignored while still writ- 
ing a large percentage of real $Z$ events to tape. One of the most pervasive sources of noise in the SLD detector are the beam halo muons.

\subsubsection{Muons}

When off-course electrons or positrons scrape any material in the transport lines, they can create high energy photons, which may in turn scatter from nuclei to create muon pairs or pions which decay into muons. These muons (called beam halo muons) can be trapped in the beam transport at large radii and accompany the parent beam all the way to the SLC interaction point. They then pass through the SLD parallel to the beam line and deposit energy in both the endcap and barrel sections of the LAC.

A selection of luminosity monitor triggers is used as a luminosity-weighted random sample of LAC backgrounds, in order to estimate the muon rate and create an SLC muon pattern recognition algorithm [58]. Since the halo muons travel parallel to the beam axis, they can deposit several $\mathrm{GeV}$ in the calorimeter, but distributed over many towers. The typical muon energy distribution is around 20 to 30 ADC counts per tower. The LAC ENERGY trigger takes advantage of these characteristics in order to minimize the number of events triggered by beam halo muons.

\subsubsection{LAC ENERGY Trigger}

The LAC ENERGY trigger relies on a set of trigger sums in order to decide whether the contents of the LAC should be written to tape. There are two types of sums, the LO sums and the HI sums. In order to be included in the HI trigger sum, tower ADC counts must be over thresholds of $60 \mathrm{ADC}$ counts in the EM sections and $120 \mathrm{ADC}$ counts in the HAD sections (since HAD towers are roughly twice the volume of EM towers). Similarly, towers are added to the LO trigger sums when they are above the LO thresholds ( $8 \mathrm{ADC}$ counts in the EM sections, 12 ADC counts in the HAD sections).

The sensitivity to muons is reduced in two ways: first, by requiring the HI sums (which are effectively blind to muons) to be above some threshold, and second, by requiring the LO sums to be below some threshold, thus cutting out events wherein too many towers are 
"lit up" by the longitudinally travelling muons. The specific trigger sums used are the following:

- EHI: the sum of energy in all towers above the HI threshold

- ELO: the sum of energy in all towers above the LO threshold

- NLO: the number of towers above the low threshold

- NEMHI: the number of towers in the EM section above the high threshold The final ENERGY trigger requires that EHI be greater than $8 \mathrm{GeV}$, and that NEMHI be greater than or equal to ten towers. The trigger is vetoed if NLO is greater than 1000 towers. During the 1993 run, approximately three million events satisfied the ENERGY trigger.

\subsection{Selection Criteria}

Three million events is clearly an unwieldy number to reconstruct, especially given that the total $Z$ content of these triggers is on the order of $2 \%$. In order to narrow down the number of potential candidates, the trigger sum requirements are tightened to form the so-called Pass One selection. Then, given the nature of the SLD backgrounds, we construct a second set of criteria, called Pass Two, which acts on reconstructed data to select a pure sample of hadronic $Z$ decays. This approach to LAC event selection was first described in Reference [56].

\subsubsection{Pass One Selection}

The Pass One selection operates on the raw trigger sums written with the events that have satisfied the ENERGY trigger described above. Pass One "tightens" the ENERGY trigger by placing the following additional requirements on the trigger sums:

- $\mathrm{ELO}<140 \mathrm{GeV}$

- $\mathrm{EHI}>15 \mathrm{GeV}$

- $\mathrm{EHI}>1.5 \cdot($ ELO - 70) 


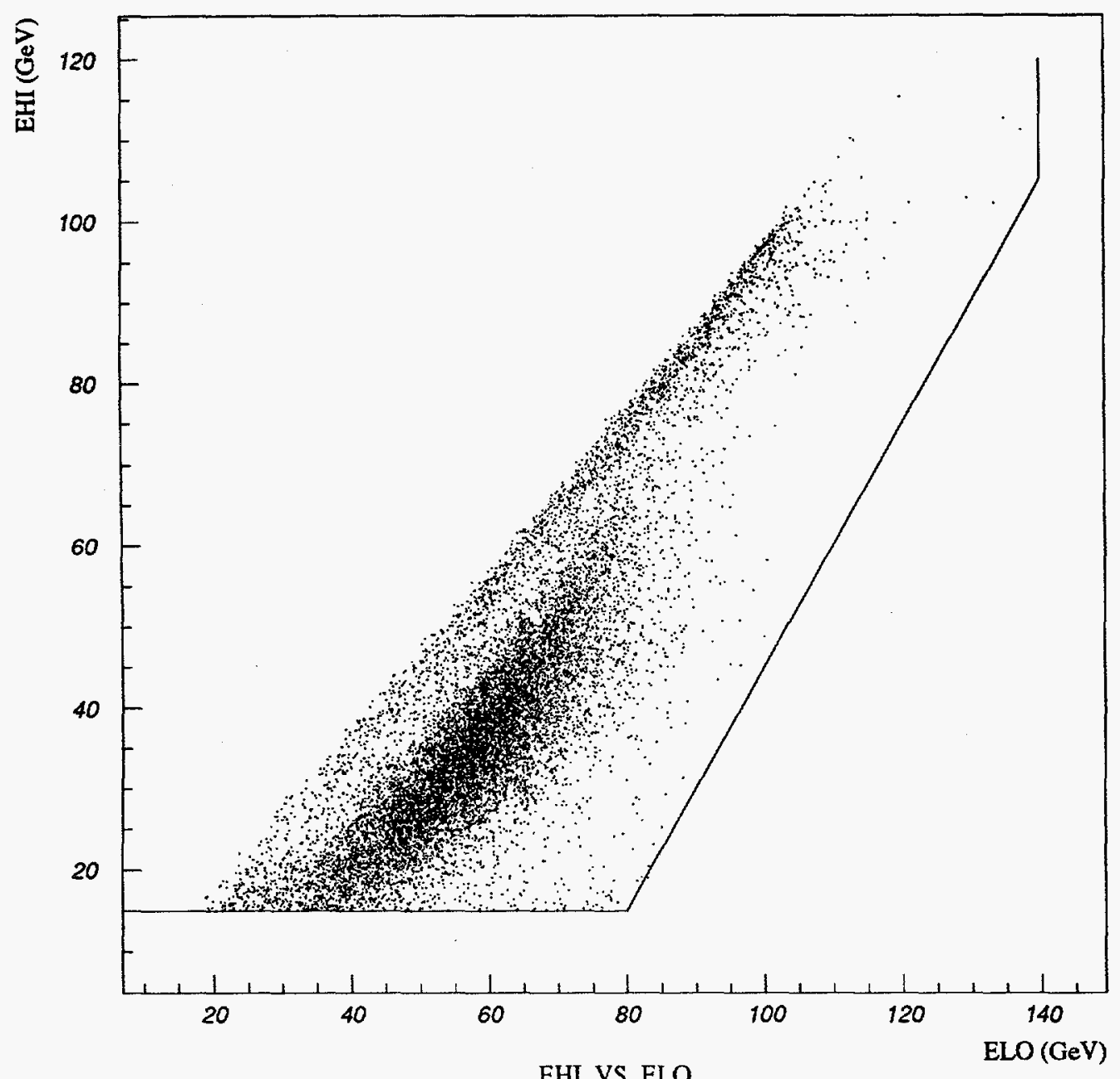

Figure 4-1: Pass One events in EHI-ELO space

Figure 4-1 shows a scatter plot of EHI versus ELO for the Pass One events ${ }^{1}$. The energy cuts are shown as solid lines. Application of this selection reduces the data sample to 63,552 events, from which we wish to distinguish the $Z$ signal.

1. This scatter plot and all other scatter plots in this chapter contain a random subsample of approximately $25 \%$ of the data, in order to avoid saturating the resolution of the plots. Of course all analyses were applied to the full data sample. 


\subsubsection{Event Reconstruction}

While the 1992 event selection was based purely on raw calorimeter data, the 1993 selection uses a reconstruction algorithm on the raw data to form vector energy clusters, on which straightforward and intuitive cuts are made.

All Pass One events are processed through the calorimeter reconstruction. Only towers with more than $7 \mathrm{ADC}$ counts in the EM section and $9 \mathrm{ADC}$ counts in the HAD section are included in the algorithm. Towers closest to the beampipe are ignored. The reconstruction groups the tower hits into clusters, based on the spatial association of the hits. In the first stage, all contiguous hits are associated into coarse clusters. During the second stage, the algorithm searches the coarse clusters for minima, breaking these clusters into smaller refined clusters if it appears as though the energy profile is the result of more than one incident particle. A refined cluster is intended to correspond to a single particle.

In order to be counted as a final cluster, a refined cluster must have the following characteristics:

- not tagged by the SLC muon recognition algorithm

- total cluster energy $>100 \mathrm{MeV}$

- total energy in EM sections $>0 \mathrm{MeV}$

The total energy and energy-weighted mean $\phi$ and $\theta$ are calculated for each final cluster,

forming a list of energy vectors, $\vec{E}_{i}$. The total number of final clusters is referred to the cluster multiplicity, $N_{\text {clus }}$.

\subsubsection{Pass Two Selection}

We must now consider the calorimeter signature of signal events. Leptonic decays each have a distinct signature. Bhabhas deposit the most energy in the LAC of any event type, and each hit is usually concentrated in one or two towers of the EM sections. Muons are sufficiently long-lived that they decay outside of the detector. They deposit little or no energy in the LAC, leaving their signature in the WIC instead. However, muon pairs represent only $3 \%$ of the $Z$ decays. Taus on the other hand decay almost immediately and always have neutrinos in the final state. Tau events often have a large missing energy com- 
ponent, and also low cluster multiplicity. Hadronic $Z$ decays are distinguished by a large number of charged tracks, which translates into a high cluster multiplicity, and by the deposition of tens of GeV in the LAC. As the primary decays do not involve neutrinos, much of the total energy is visible in the calorimeter.

The visible energy of a $Z$ decay event is a significant fraction of the $Z$ mass. In order to reject low energy backgrounds, such as two-photon events and beam-related backgrounds (see section 4.4 for details), it is required that all events contain at least $15 \mathrm{GeV}$ (minimum ionizing energy scale) of total observed energy, $E_{t o t}$.

Furthermore, the total momentum of an event arising from electron-positron scattering (as apposed to, for instance and event in which an electron scatters off a stationary target such as the beampipe) should be zero. We therefore define a dimensionless calorimeter quantity $I$, called the energy imbalance, which is related to the momentum sum:

$$
I=\frac{\left|\sum_{\text {clusters }} \vec{E}_{i}\right|}{\sum_{\text {clusters }}\left|\vec{E}_{i}\right|}
$$

where $\vec{E}_{i}$ is the energy vector of the $i$ th cluster.

For $Z$ events with all energy visible in the calorimeter, $I$ should be zero. Events resulting from beam interactions with residual gases in the beampipe or structures near the IP are highly boosted in the laboratory frame, which is to say, they have a high energy imbalance. Most of this energy is lost down the beam pipe. Similarly, most cosmic ray events deposit energy in only one hemisphere of the detector and will therefore also have a large energy imbalance. The imbalance threshold has been chosen at $I=0.6$. Figure $4-2$ shows the Pass One events in $E_{\text {tot }}$ versus I space, with the solid lines showing the cuts. The points with high $E_{\text {tot }}$ and low $I$ are the Bhabhas, which, as noted previously, deposit the most calorimeter energy of any event type.

The energy and imbalance cuts do not remove the Bhabha final states, for the very good reason that these are real $Z$ decays. Nevertheless, these events must be considered a background to the $A_{L R}$ analysis. Bhabha scattering occurs through both $Z$ and photon exchange, 


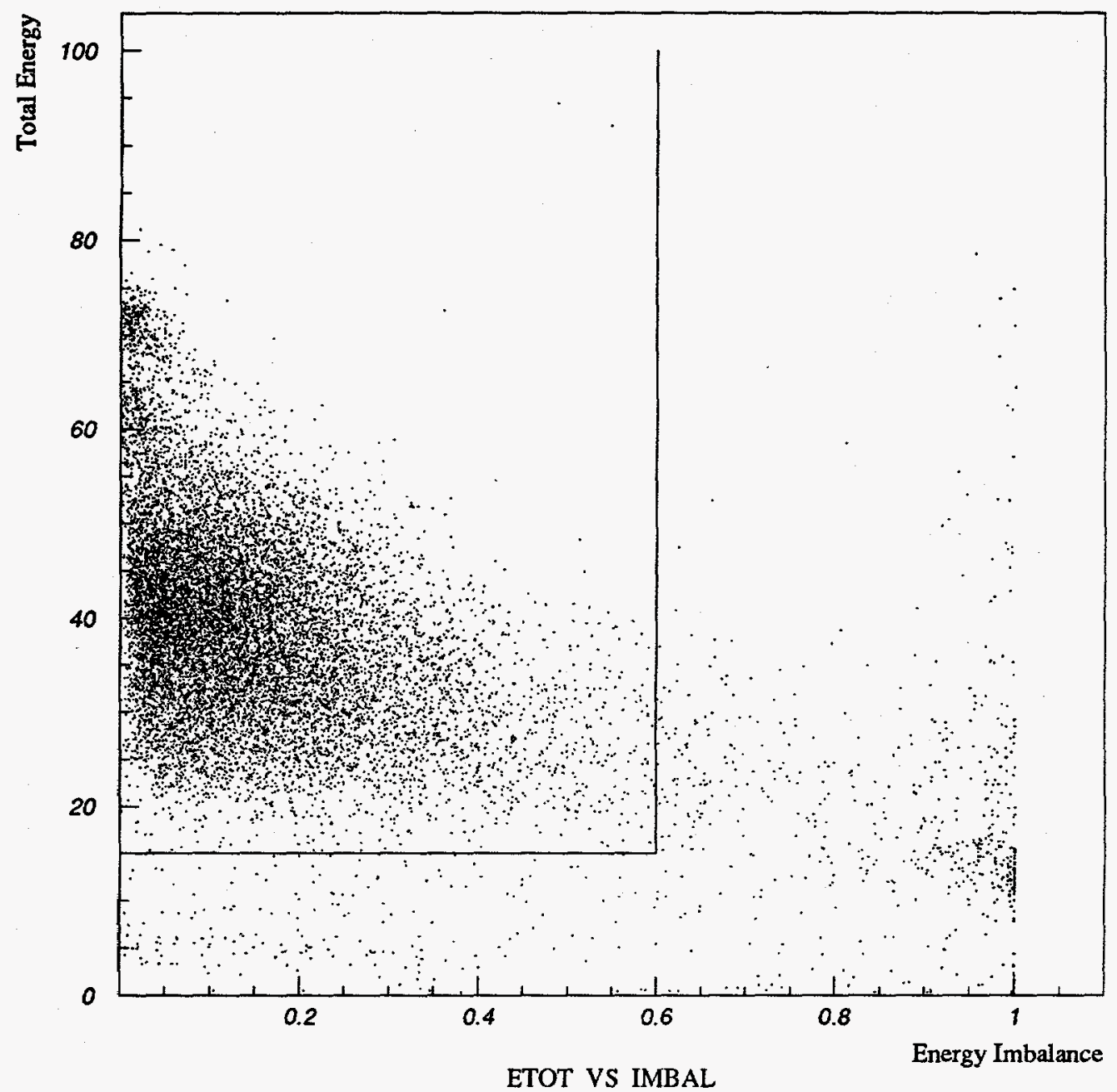

Figure 4-2: Pass One events in Energy versus Imbalance space

as illustrated in figure 4-3. The photon exchanges, of which the $t$-channel process dominates the cross section at small scattering angles, do not exhibit the asymmetry characteristic of the $Z$ exchange. Thus the asymmetry of Bhabha events is diluted as a function of angle, and cannot be included in the hadronic event sample.

The cluster multiplicity in these events is very low because high-energy electrons (or positrons) tend to deposit all of their energy in a single tower of the electromagnetic section 


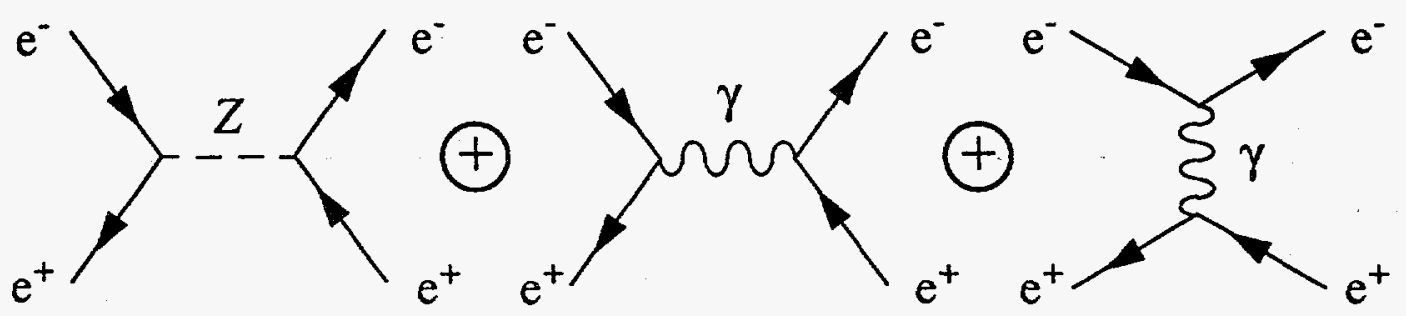

Figure 4-3: Tree-level Bhabha scattering

of the calorimeter. However, since the response of the calorimeter changes as $\cos \theta$ increases, primarily due to pre-showering in inner detector material [59], and since the Bhabha background increases rapidly with $\cos \theta$, separate cluster cuts are applied to different ranges of polar angle. Trial and error shows the best separation for each sub-sample occurs around the $(\cos \theta=0.8)$ boundary. Therefore, the following cuts are defined for the two different regions:

$$
\begin{array}{ll}
0.0 \leq|\cos \theta|<0.8 & N_{\text {clus }}>8 \\
0.8 \leq|\cos \theta| \leq 1.0 & N_{\text {clus }}>11
\end{array}
$$

The $(\cos \theta=0.8)$ boundary forms the logical definition of the barrel region and endcap region, which will be referred to throughout this chapter, and which do not exactly correspond with the physical regions, whose boundary occurs around $\cos \theta=0.7$. Figure 4-4 shows a summary of the Pass Two calorimeter cuts. In each one-dimensional projection, all other cuts have already been applied.

\subsubsection{Polarimeter Cuts}

There are two final cuts on the event sample related to the polarization data. Each $Z$ candidate is matched with a polarization by finding the valid polarimeter measurement most closely associated in time with the $Z$ event. We require that this polarization magnitude be greater than $10 \%$, which has the effect of removing $30 \mathrm{Zs}$ from the beginning of the run, during which the beams were unpolarized. In addition we require that the polarimeter run be within 0.0415 days ( $\approx 1$ hour) of the event. This cut removes $1285 \mathrm{Zs}$ from the final sample. This measures the polarimeter live-time to be $(50677-1285) / 50677=97.5 \%$. It will be shown in Chapter 5 that the measured asymmetry is unbiased by this time cut. 

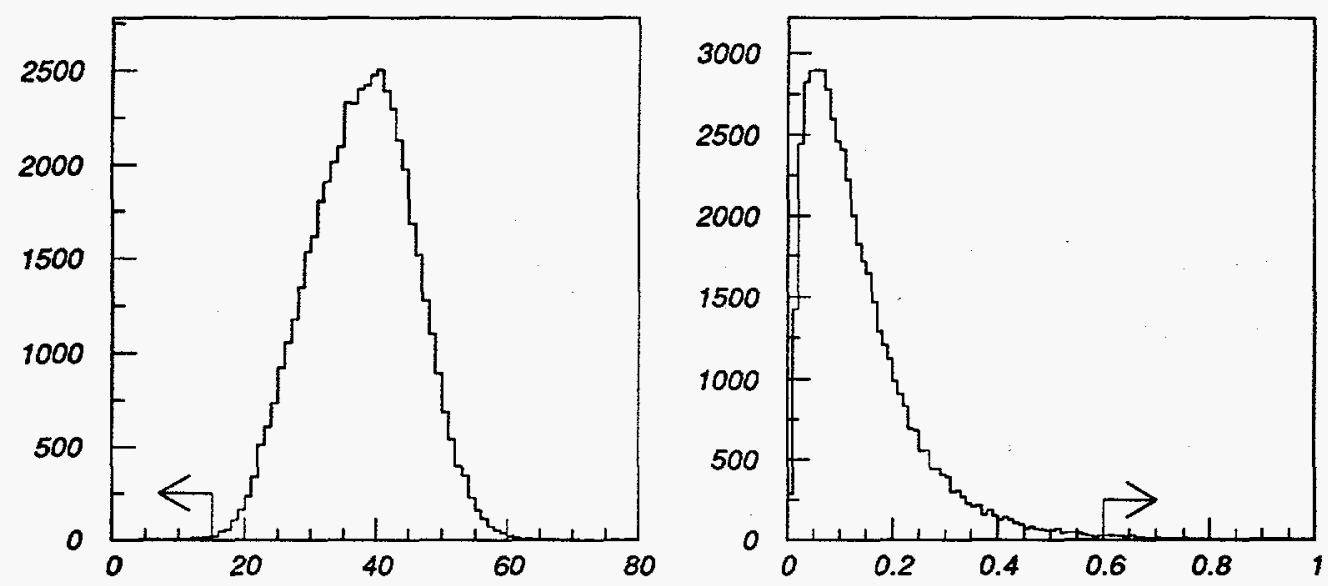

Total Energy

Energy Imbalance

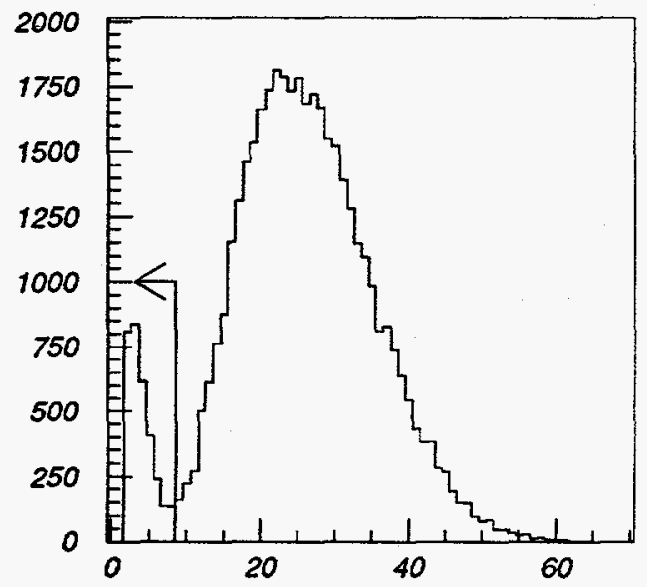

Cluster Multiplicity (cth less than 0.8)

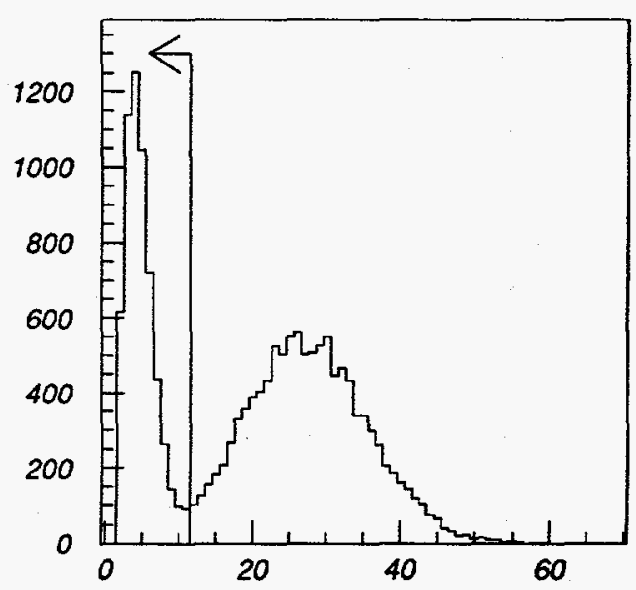

Cluster Multiplicity (cth greater than 0.8)

Figure 4-4: A summary of the Pass Two event selection. 


\subsubsection{Result}

A total of 49,392 primarily hadronic $Z$ events remain after the Pass Two selection and polarimeter cuts, of which 27,225 are associated with a left-handed electron beam, and 22,167 are associated with a left-handed electron beam. The measured asymmetry $A_{N}$ is then

$$
A_{N}=\frac{N_{L}-N_{R}}{N_{L}+N_{R}}=0.1024 \pm 0.0045
$$

where the error quoted is a statistical only. Extracting $A_{L R}$ from this measured asymmetry will be the subject of Chapter 5 .

\subsubsection{Tracking-based Event Selection}

It can be argued that a tracking-based event selection is a much cleaner and more straightforward method for an $A_{L R}$ analysis. The backgrounds for such a selection are less than $0.1 \%$, with an even smaller associated systematic error [60]. However, because noisy SLC conditions force the drift chambers to be off or under-voltage for non-negligible periods of time, and because of the inherent dead-time associated with the drift chamber data acquisition, the use of tracking information incurs a $20 \%$ loss in statistics.

Nevertheless, we present a very simple tracking-based analysis here as a cross-check on the calorimeter analysis. From the Pass One events with tracking information available, tracks are required to have the following properties:

- momentum $\geq 250 \mathrm{GeV}$

- $\rho<5 \mathrm{~cm}$

- $|z|<5 \mathrm{~cm}$

where $\rho$ is the transverse distance between the track's point of closest approach to the nominal IP and the beam axis, and $z$ is the distance from the point of closest approach to the IP along the beam axis. The total number of tracks that meet these requirements is denoted by $N_{\text {track }}$. The tracking-based event selection then simply consists of all events with $N_{\text {track }} \geq 6$. 
Of the events which meet this requirement, we find 20867 left-handed events and 16974 right-handed events, for a measured asymmetry of

$$
A_{N}^{\text {track }}=0.1029 \pm 0.0051 .
$$

This number agrees with the calorimetric data selection measured asymmetry of 0.1024 , within the statistical errors (the errors are correlated, since the tracking events are almost entirely a subsample of the calorimeter events). The tracking data selection is susceptible to different backgrounds, and is entirely free of the Bhabha background which dominates the calorimeter analysis. The agreement confirms that the result of the calorimeter analysis is not an artifact caused by backgrounds peculiar to that data selection.

\subsection{Background Estimation}

Events that do not arise from $Z$ decays do not have an inherent asymmetry, and therefore dilute the measured asymmetry if included in the final sample. We require a quantitative estimate of the total background in order to correct the measured asymmetry for this effect and to estimate the associated systematic uncertainty. There are several types of backgrounds to consider but their effects on the measured asymmetry are very small. The first comprehensive work on LAC backgrounds is found in Reference [61]. The updated 1993 background analysis is documented in Reference [62], from which this description follows.

In the following sections, the estimated number of background events is referred to as $N_{b}$, and the resulting background fraction is referred to as $f_{b}$. The background contributions from different sources (subsamples of $N_{b}$ and $f_{b}$ ) are identified by superscripts.

\subsubsection{Bhabhas}

As noted previously, Bhabha events have a $\theta$ dependent asymmetry, and are therefore must be removed from the analysis. However, the separation between Bhabhas and hadronic events in the cluster multiplicity space is not great (as illustrated in figure 4-4). As a result, residual Bhabhas are the largest background to the $A_{L R}$ analysis. However, because these events do have an asymmetry, the resulting background correction to the measured 
asymmetry in somewhat mitigated. These background events are also referred to as the WABs (wide-angle Bhabhas), which distinguishes them from the Luminosity Bhabhas (low-angle Bhabhas detected by the Luminosity Monitor).

Bhabhas are characterized by large energy depositions in the EM section of the LAC. The clusters appear back-to-back, which is to say, the events have a low energy imbalance. The fact that the energy deposition is usually concentrated in a few towers is used to remove a majority of the Bhabhas from the sample, through the $N_{c l u s}$ cut. We use a Monte Carlo simulation to estimate the efficiency of the $N_{\text {clus }}$ cut and thereby the number of Bhabhas that are not removed by this cut.

Unfortunately, the present LAC Monte Carlo simulation, which has been well-tuned for the energy versus $\cos \theta$ distribution, does a poor job of reproducing the $N_{\text {clus }}$ distributions. Results from the simulation are shown in figure 4-5. Hadronic $Z$ events were reconstructed with the identical algorithm described in section 4.3.2., and the cluster multiplicity is overestimated in both regions of the detector. Bhabhas were reconstructed with the same algorithm, with one slight change: the minimum energy required for a cluster to be counted as a final cluster was raised from $100 \mathrm{MeV}$ to $200 \mathrm{MeV}$. This has the effect of modelling the cluster multiplicity well in the endcap region, but poorly in the barrel region.

These results are indicative of the primary problem with the detector simulation: the addition of extra material necessary to properly model the energy distribution causes additional fragmentation of the jets, which in turn leads to too many reconstructed clusters. It therefore seems sensible to use energy-related quantities, for which the Monte Carlo has been tuned, in an attempt to isolate the Bhabhas and estimate the sample contamination.

\section{Method 1}

This approach is based on the following observation: Bhabhas tend to deposit most or all of their energy in only a few towers in the EM section of the calorimeter. Other final states (including most taus) have more energy in the hadronic section, and this energy is spread over more towers. 


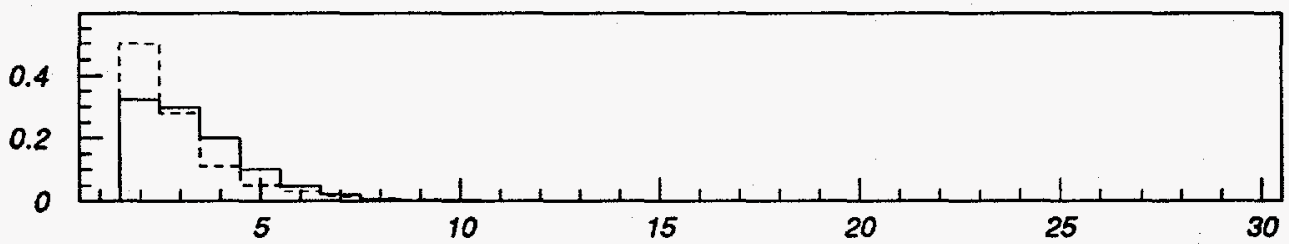

Bhabha Cluster Multiplicity (Barrel)

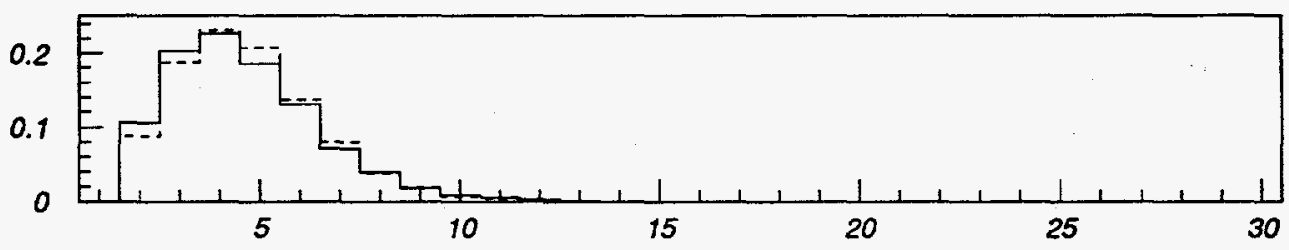

Bhabha Cluster Multiplicity (Endcap)

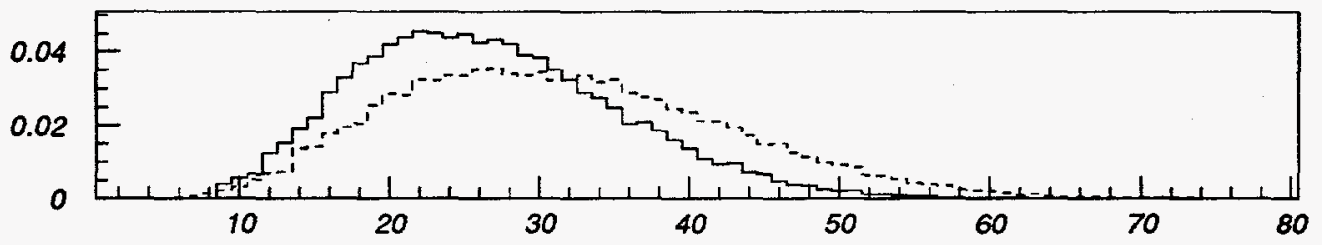

Hadronic Cluster Multiplicity (Barrel)

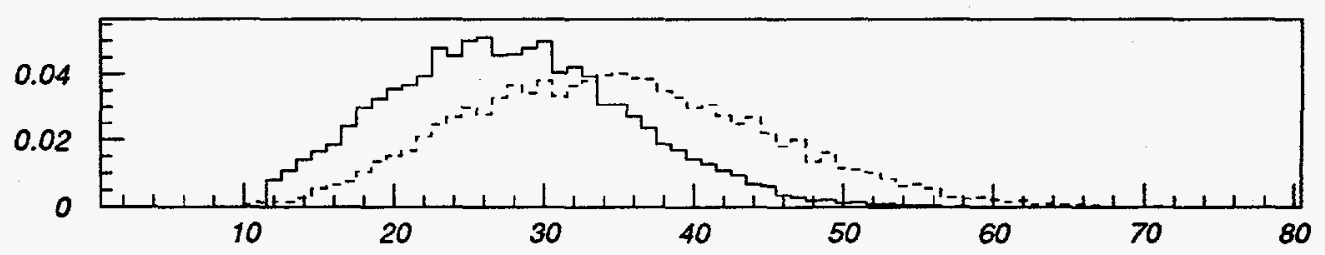

Hadronic Cluster Multiplicity (Endcap)

Figure 4-5: Normalized cluster multiplicity distributions for Bhabha and hadronic events.

Solid lines are data, dotted lines are Monte Carlo. 
These observations lead us to select the following quantities:

- the total HAD energy of an event (EHTOT)

- the EM energy in the two highest-energy clusters (EEHI1+EEHI2)

A scatter plot of the data in this variable space is shown in figure 4-6 for the barrel and endcap regions. Note that the total energy and energy imbalance cuts have already been applied to this data.

The lines in figure 4-6 separate region A, the region that should be predominantly Bhabhas, from region $B$, the region that should be mostly hadronic $Z s$. These regions have different definitions in the barrel and endcap, specifically:

$$
\begin{aligned}
& \text { Barrel region A: }(\text { EEHI } 1+\text { EEHI2 })>(40+8 \cdot \text { EHTOT }) \\
& \text { Barrel region B: }(\text { EEHI }+ \text { EEHI })<(40+8 \cdot \text { EHTOT }) \\
& \text { Endcap region A: }(\text { EEHI } 1+\text { EEHI } 2)>(20+13.33 \cdot \text { EHTOT }) \\
& \text { Endcap region B: }(\text { EEHI } 1+\text { EEHI })<(20+13.33 \cdot \text { EHTOT })
\end{aligned}
$$

The Bhabha Monte Carlo distributions of EHTOT and EEHI1+EEHI2 match the data (that is, the data in region A) fairly well in both the barrel and endcap regions. The $e / \mu$ energy scaling has already been accounted for in the Bhabha Monte Carlo, whereas the hadronic Monte Carlo is based purely on the minimum ionizing energy scale, and therefore adjustments for the $e / \mu$ and $\pi / \mu$ scaling must be made (r.e. section $4-1)$. The hadronic Monte Carlo distribution of EHTOT matches the data (in region B) well in both the barrel and endcap regions when the energy is scaled by a factor of 1.316 . The hadronic Monte Carlo distribution of EEHI1+EEHI2 matches the data (in region B) well in both the barrel and endcap regions when the energy is scaled by a factor of 1.202 . These normalized distributions are shown in figure 4-7, where the solid line represents data and the dashed line is Monte Carlo.

The results from the data and Monte Carlo simulations are summarized in tables 4-1 and 4-2. The number of Monte Carlo events has been scaled to match the data in each region. The subscripts $W$ and $H$ refer to Bhabha (WAB) and hadronic samples, respectively, while $A$ and $B$ refer to the regions defined in equation (4.4) above. 

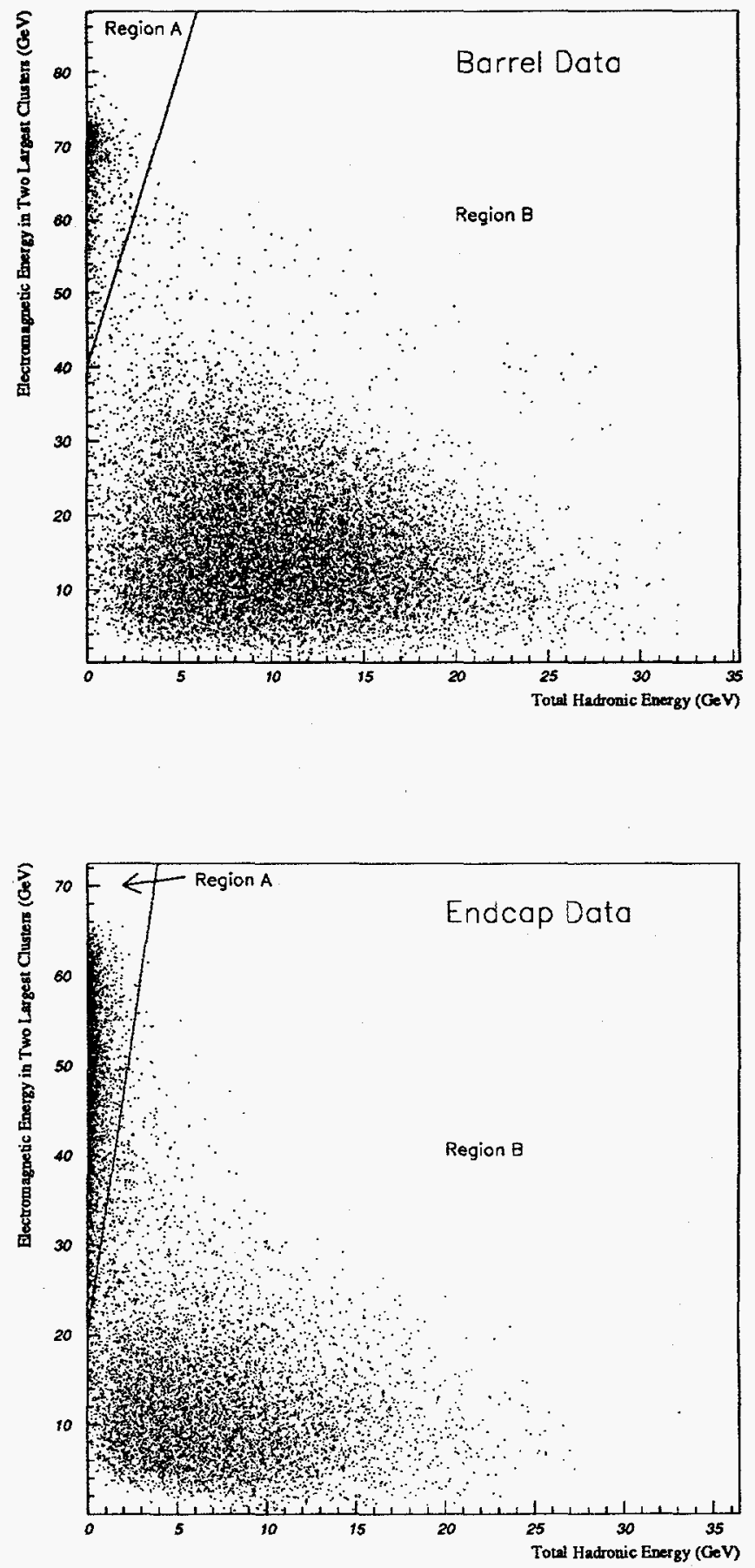

Figure 4-6: Bhabha selection Method 1 


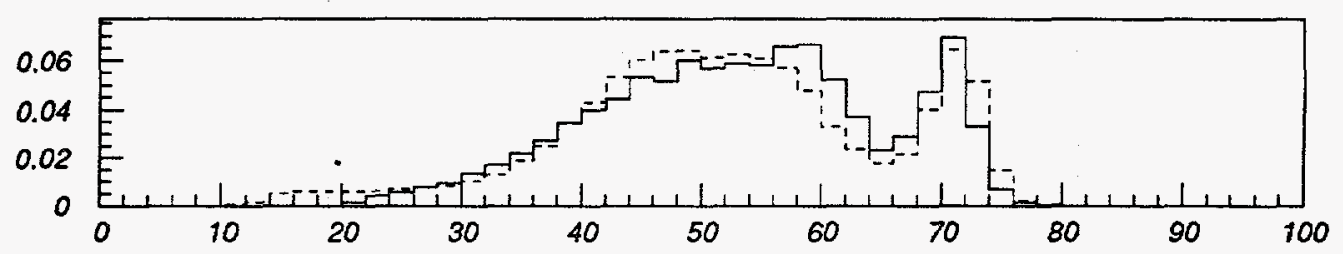

Bhabha EEHI1+EEHI2 distribution

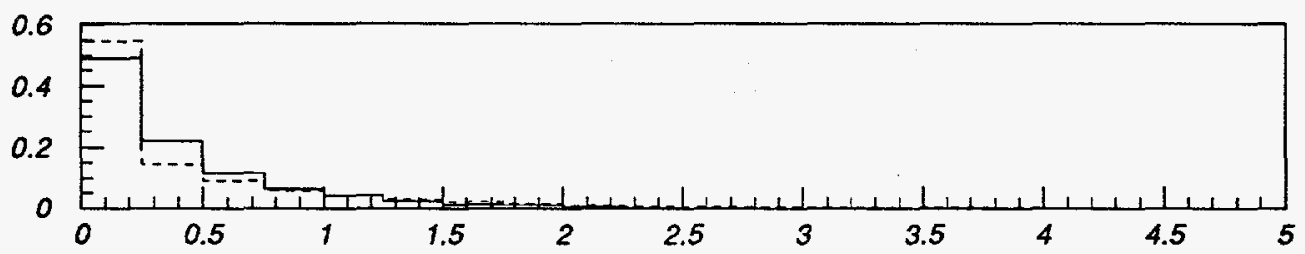

Bhabha EHTOT distribution

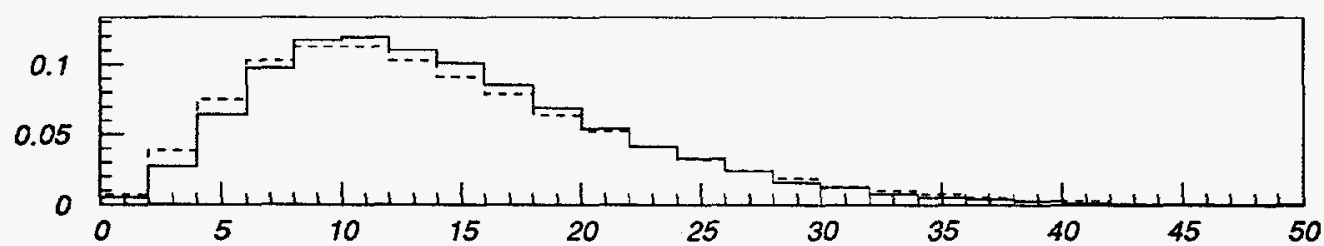

Hadronic EEHI1+EEHI2 distribution

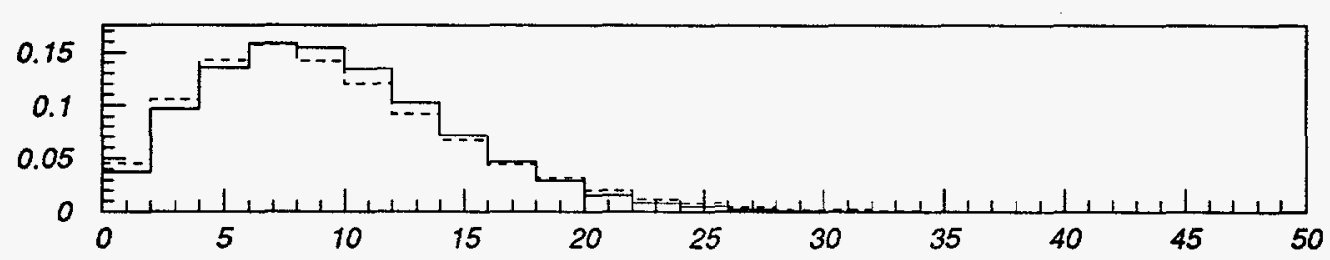

Hadronic EHTOT distribution

Figure 4-7: The distributions of EEHI1+EEHI2 and EHTOT for barrel and endcap regions; both data and normalized Monte Carlo are shown. 


\begin{tabular}{|c|c|c|c|c|}
\hline Quantity & $\begin{array}{c}\text { Monte Carlo } \\
\text { pre } N_{\text {clus }} \text { cut }\end{array}$ & $\begin{array}{c}\text { Monte Carlo } \\
\text { post } N_{\text {clus }} \text { cut }\end{array}$ & $\begin{array}{c}\text { Data } \\
\text { pre } N_{\text {clus }} \text { cut }\end{array}$ & $\begin{array}{c}\text { Data } \\
\text { post } N_{\text {clus }} \text { cut }\end{array}$ \\
\hline \hline$N_{W, A}$ & 2005 & 6.1 & 2005 & 8 \\
\hline$N_{H, A}$ & 9.6 & 9.6 & & \\
\hline$N_{W, B}$ & 60.2 & 1.8 & & 39689 \\
\hline$N_{H, B}$ & 39794 & 39689 & 40861 & \\
\hline
\end{tabular}

Table 4-1: Results of Method 1 Bhabha background estimate: Barrel Region

\begin{tabular}{|c|c|c|c|c|}
\hline Quantity & $\begin{array}{c}\text { Monte Carlo } \\
\text { pre } N_{\text {clus }} \text { cut }\end{array}$ & $\begin{array}{c}\text { Monte Carlo } \\
\text { post } N_{\text {clus }} \text { cut }\end{array}$ & $\begin{array}{c}\text { Data } \\
\text { pre } N_{\text {clus }} \text { cut }\end{array}$ & $\begin{array}{c}\text { Data } \\
\text { post } \mathrm{N}_{\text {clus }} \text { cut }\end{array}$ \\
\hline \hline$N_{W, A}$ & 4619 & 5.5 & 4619 & 41 \\
\hline$N_{H, A}$ & 19.6 & 19.6 & & \\
\hline$N_{W, B}$ & 560.2 & 2.0 & & 10969 \\
\hline$N_{H, B}$ & 10998 & 10969 & 12195 & \\
\hline
\end{tabular}

Table 4-2: Results of Method 1 Bhabha background estimate: Endcap Region

In the endcap, and after the cluster multiplicity cut, there were 41 events remaining in the Bhabha region (region A) of the energy space shown in figure 4-6. We hypothesize that these events are WABs, but there are in fact hadronic events that spill over from region $B$ into region $A$, so these events are actually a mixture of WABs and hadronic $Z$ decays. We use the hadronic Monte Carlo to estimate the hadronic contamination in region $\mathrm{A}$, which is predicted to be 19.6 events. We also need to know if there are any hidden background events, that is, WABs occurring in the hadronic region (region B) of the energy space. The Bhabha Monte Carlo estimates 2.0 events of this type. Thus the estimate for Bhabhas in the endcap region is 41 observed events plus 2 hidden events, minus an expected background of 19.6 events, for a total of 23.4 events.

The same procedure is applied to the barrel, in which 8 events are observed in region $\mathrm{A}$ after the cluster multiplicity cut. The respective Monte Carlo simulations predict 9.6 events 
as hadronic contamination in region $\mathrm{A}$, and 1.8 events as the hidden wide-angle Bhabha background in region $B$.

We are interested in an upper bound on the Bhabha contamination from these predictions. To summarize:

Barrel: $\quad 8$ observed +1.8 hidden, background of 9.6: $\quad 7.45$ events at $95 \%$ c.l.

Endcap: 41 observed +2.0 hidden, background of 19.6: 35.85 events at $95 \%$ c.l.

Total WAB background $=(7.45+35.85) /(49392)=0.000854$

Which is to say, the total background fraction due to wide-angle Bhabhas, $f_{b}{ }^{W}$, is less than $0.09 \%$ at the $95 \%$ confidence level. This result can also be expressed in terms of a central value symmetrized about the $16.6 \%$ and $83.3 \%$ confidence contours:

$$
\begin{aligned}
& N_{b}^{W}=27.0 \pm 8.8 \\
& f_{b}^{W}=(0.053 \pm 0.017) \%
\end{aligned}
$$

A major drawback in this method is the reliance on the Monte Carlo prediction of the hidden backgrounds; that is, those Bhabhas that remain in the hadronic region (region B) after the cluster multiplicity cut. Specifically, the Bhabha cluster multiplicity is underestimated by the Monte Carlo, which means that the hidden background $N_{W, B}$ is likely to be underestimated in the barrel region. Additionally, the Bhabha Monte Carlo does not reproduce certain types of observed events wherein a Bhabha slips through a crack and deposits significant energy in the hadronic section of the calorimeter, which again implies an underestimate of the hidden background.

\section{Method 2}

An alternative technique for extracting the WAB background was independently developed from that described above. The approach, however, is essentially the same: Develop criteria to further isolate the WAB background within the $A_{L R}$ sample in such a way that $f_{b}{ }^{W}$ can be reliably estimated.

The quantity used here to perform the additional separation is the ratio $R$ of the energy in all LAC electromagnetic clusters (EETOT) to the total LAC cluster energy (ETOT). Fig- 
ure 4-8 (A) shows the distribution of $R$ for the data (Pass One plus the energy and imbalance cuts). The Bhabhas are clustered tightly about $R=1$, but there is not very good separation between the WABs and the hadronic events. We therefore invent a new ratio R1, which is the sum of all electromagnetic energy in clusters with greater than $1 \mathrm{GeV}$ total energy, divided by the total energy in the event. Figure 4-8 (B) gives the distribution of R1 for the data. We see that the WAB-hadron separation with this modified electromagnetic ratio is greatly improved. This is because hadronic events include of a relatively large number of low-energy electromagnetic clusters compared to WABs.

Using the $1 \mathrm{GeV}$ cluster threshold, we may also define a new cluster multiplicity, $N_{c l u s, 1}$, which is always less than or equal to $N_{\text {clus. }}$. Figure 4-8 (C) shows the distribution of $N_{c l u s, 1}$ for the data (that is, for Pass One events that pass the energy and imbalance cuts, but fail the cluster multiplicity cuts) as a solid histogram, and the Bhabha Monte Carlo overlaid as a dotted histogram. The agreement is so good that the Monte Carlo is barely visible. This is consistent with the hypothesis that the problem with modelling $N_{c l u s}$ arises in the low energy fragmentation in the Monte Carlo. In any case both data and Monte Carlo suggest that more than $99 \%$ of all WABs have $N_{\text {clus }, 1}$ less than 6 . Figure 4-8 (D) shows the data with the Pass Two energy and imbalance cuts, plus an $N_{\text {clus, }, 1}<6$ cut, from which a WAB threshold cut of $R 1>0.86$ is derived. Figure 4-8 (E) shows the R1 distribution for Pass Two events with $N_{c l u s, 1}$ less than 6 , with the backgrounds selected for $\mathrm{R} 1>0.86$. Thus in the Pass Two data sample, $0.34 \%$ (172/50707) of the events are found by the Method 2 Bhabha selection. It is clear from 4-8 (E) that not all selected events are WABs, so the remaining question is what fraction of the $0.34 \%$ is due to non-WABs. Both tau and hadron events are expected to contribute at a low level.

Based on $\sigma_{\tau}=1.41 \mathrm{nb}$, we expect about 2550 tau events produced in the 1993 run. With the $1 \mathrm{GeV}$ cluster threshold cut discussed above, tau Monte Carlo predicts 61 tau events which are mis-identified as WABs. This corresponds to $0.12 \%$ of the $A_{L R}$ sample. Subtracting this from above gives $f_{b}{ }^{W}=(0.22 \pm 0.05) \%$

To get an idea of how many hadron events are mis-identified as WABs, tracking information is examined where available. The events selected by Method 2 as WAB candidates 


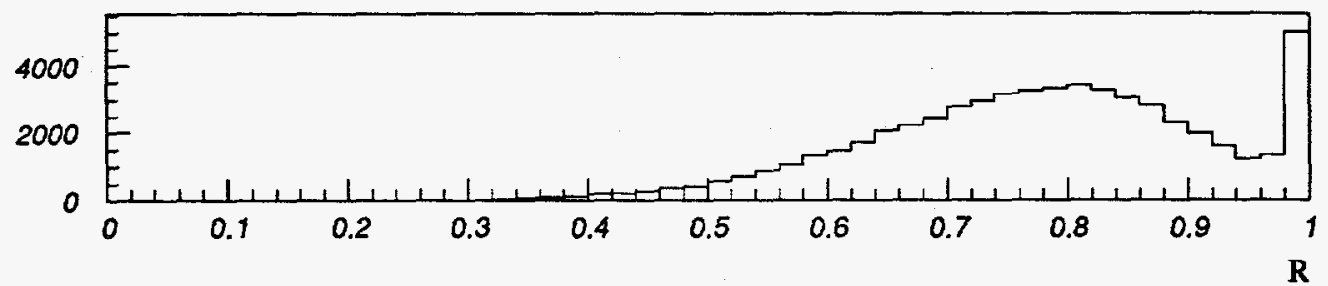

(A)

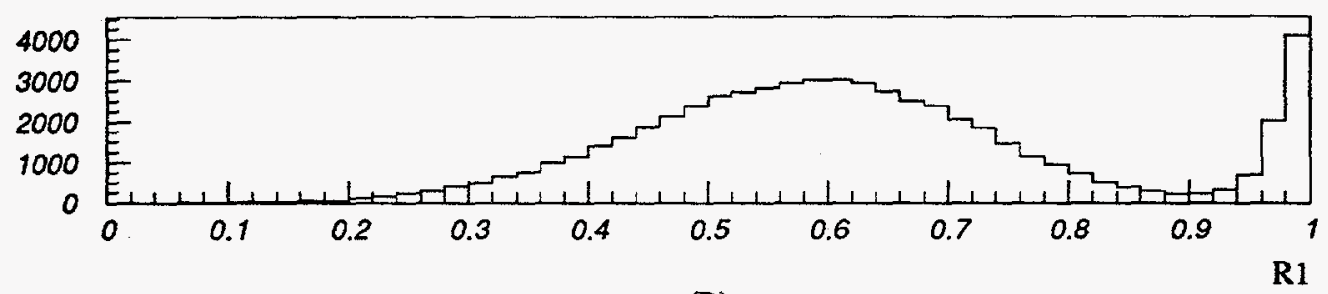

(B)
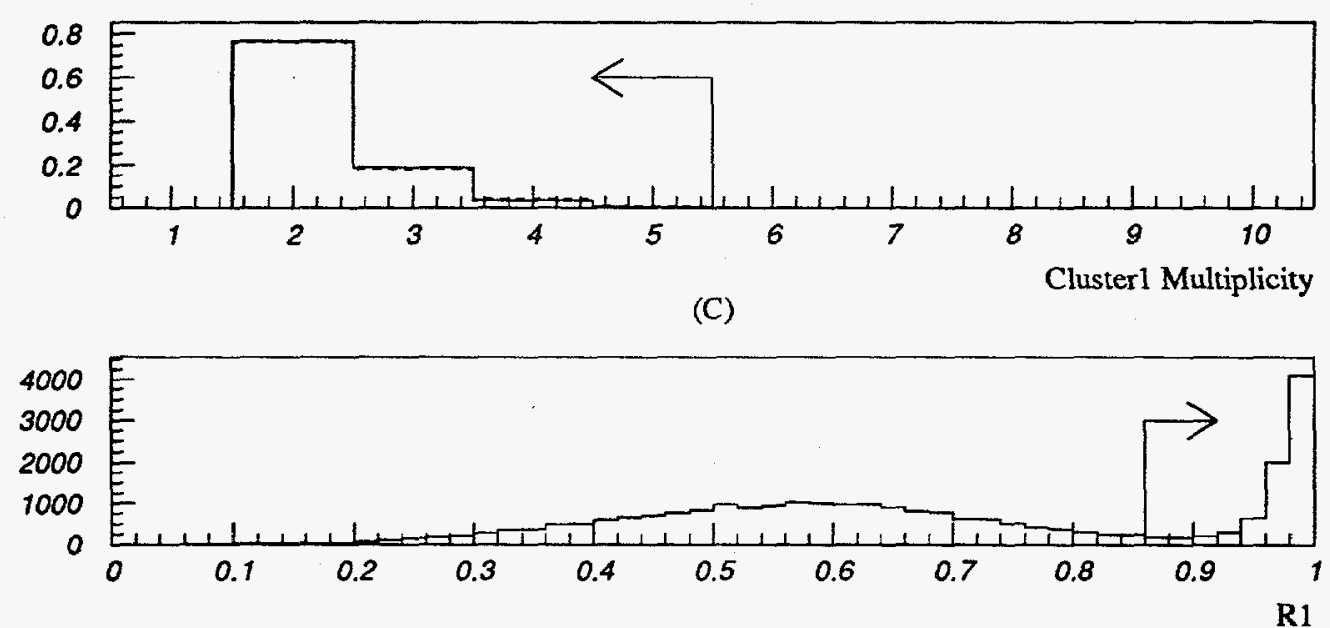

(D)

R1

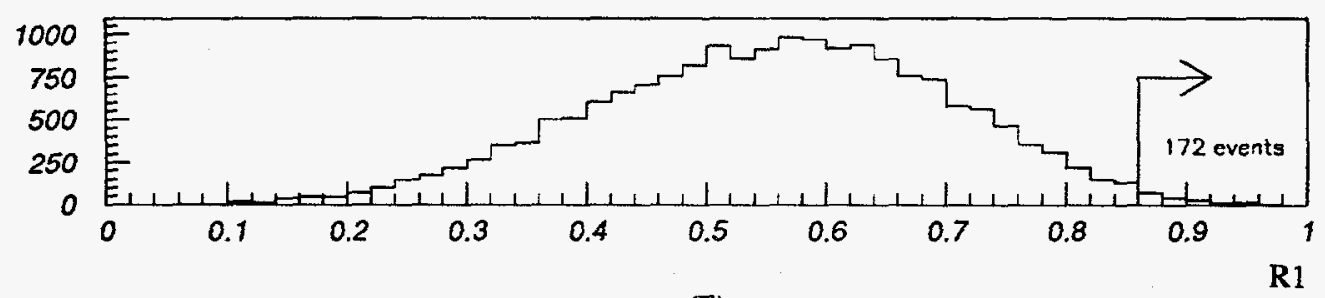

(E)

Figure 4-8: Bhabha selection Method 2

(complete description in text) 
were examined for their CDC tracking content. 58\% of the events did have CDC information. Of these events, the majority, $63 \%$, have more that 6 good tracks, and hence are primarily hadronic events. That is to say, that at least $36 \%$ of the estimate are definitely not Bhabhas. Because of the uncertainty in extrapolating the track multiplicity analysis to those events without $\mathrm{CDC}$ information, we cannot make a quantitative estimate of the hadronic contamination in the endcap region. Therefore we subtract only those events positively identified as hadronic decays in the barrel region, for a final Method 2 estimate of

$$
f_{b}^{W}=(0.14 \pm 0.05) \%
$$

This estimate represents an upper limit for the WAB contamination in the $A_{L R}$ sample.

\section{Result}

It is not straightforward to reconcile the results of these two different Bhabha background estimates. Method 1 is probably an underestimate, due to its dependence on the poorly modelled cluster multiplicities needed to determine the hidden backgrounds. Method 2 on the other hand, is an overestimate, in that its selected background is certainly contaminated by real hadronic events. The final background fraction is taken to be the average between theses two:

$$
f_{b}^{W}=(0.10 \pm 0.06) \%
$$

with a systematic uncertainty inflated to account to some degree for the discrepancy between the two methods.

\subsubsection{Cosmic Rays}

It is possible for cosmic rays to deposit more than $20 \mathrm{GeV}$ in the LAC. The dominant mechanism is through the radiation of a hard photon by a cosmic muon. The effects of other types of cosmic ray particles (electrons, neutrons, protons) is approximately two orders of magnitude smaller than this muon contribution [63]. The total number of muons with energy $\geq 20 \mathrm{GeV}$ that cross the interaction point during the 1993 run is estimated to be 8400 , based on the size of the interaction point, the total live-time of the SLD detector, and 
a prediction of the flux of cosmic-ray muons [64]. Of these, the number likely to radiate more than $20 \mathrm{GeV}$ in the LAC is expected to be $\leq 2.5$.

However, in order to satisfy the energy imbalance cut, the radiation must take place on one side of the detector and shower all the way through to the other side of the interaction point. The probability for this is much less than one, and this number should be taken as an upper limit only, that is

$$
N_{b}^{C} \leq 2.5 \quad f_{b}^{C} \leq 0.005 \% \text {. }
$$

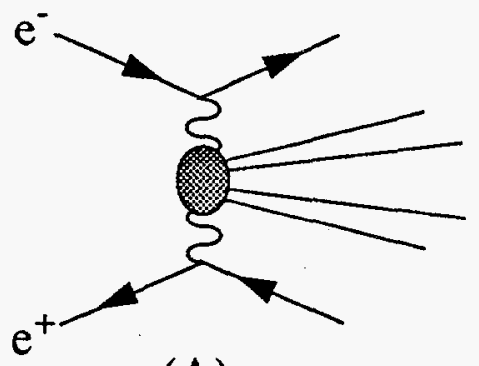

(A)

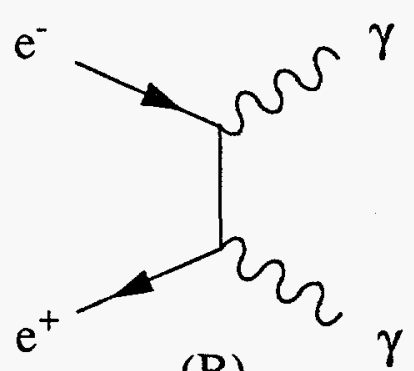

(B)

Figure 4-9: (A) The $2 \gamma$ process. (B) The $\gamma \gamma$ process.

\subsection{3 $2 \gamma$ Events}

The two-photon (or $2 \gamma$ ) process should not be confused with the gamma-gamma $(\gamma \gamma)$ process. Refer to figure 4-9 for clarification. $2 \gamma$ events are the result of the fusion of two photons that have been radiated from the incoming electrons and positrons.

In these events, the final state electron and positron usually travel unobserved down the beampipe, while the photon products deposit low and likely unbalanced energy in the LAC. Approximately $4 \mathrm{pb}^{-1}$ of $2 \gamma$ events were generated by the TPC/ $2 \gamma$ collaboration Monte Carlo [65] and put through the normal event reconstruction and filter. None of the Monte Carlo events passed the filter. This places an upper limit of 1.5 events at the $95 \%$ confidence limit on this type of background, or

$$
N_{b}^{2 \gamma} \leq 1.5 \quad f_{b}^{2 \gamma} \leq 0.003 \%
$$




\subsection{4 $\gamma$ Events}

The $\gamma$ (gamma-gamma) process is a simple QED electron exchange illustrated in figure 4-9 (B) This process has a well-understood cross section given by [66]

$$
\frac{d \sigma}{d \Omega}=\frac{\alpha^{2}}{s}\left(\frac{1+\cos ^{2} \theta}{1-\cos ^{2} \theta}\right)
$$

where $\alpha$ is the electromagnetic charge, $s$ is the center-of-mass energy-squared, and $\theta$ is the angle of the emerging photons.

Such events appear as two extremely balanced depositions of electromagnetic energy, exactly as Bhabhas (there are of course no tracks, but tracking is ignored in this analysis). We integrate equation (4.3) over the acceptance of the LAC to determine that the total cross section is $\sigma=61_{-9}^{+4} \mathrm{pb}$, which is approximately $0.1 \%$ of the total Bhabha cross section. Since these events look just like Bhabhas in the LAC, we assume that they are rejected by the cluster multiplicity cut with the same efficiency as the Bhabhas, and therefore represent a truly negligible contribution to the total background.

\subsubsection{Beam Background Events}

Beam background events include collision of beam electrons with the beam pipe, beam gas, or other apparatus. Such events are characterized by low energy deposition and a large energy imbalance. Such events are clearly visible in figure 4-2. However, one event from each beam, occurring on the same beam crossing could look like a balanced $Z$ event.

The beam background is by nature difficult to model with Monte Carlo simulation. We instead exploit the unbalanced nature of these beam related events and use the data itself to estimate the background. We estimate how many background events survive the Pass Two cuts by observing and comparing the shapes of the energy imbalance distributions in the data. First we note that the unbalanced events tend to have low cluster multiplicity. This fact is illustrated in figure 4-10. We therefore expect that the imbalance distribution for events with $N_{\text {clus }}>20$ is devoid of beam backgrounds, and provides a calibration of the shape of the background-free distribution. 


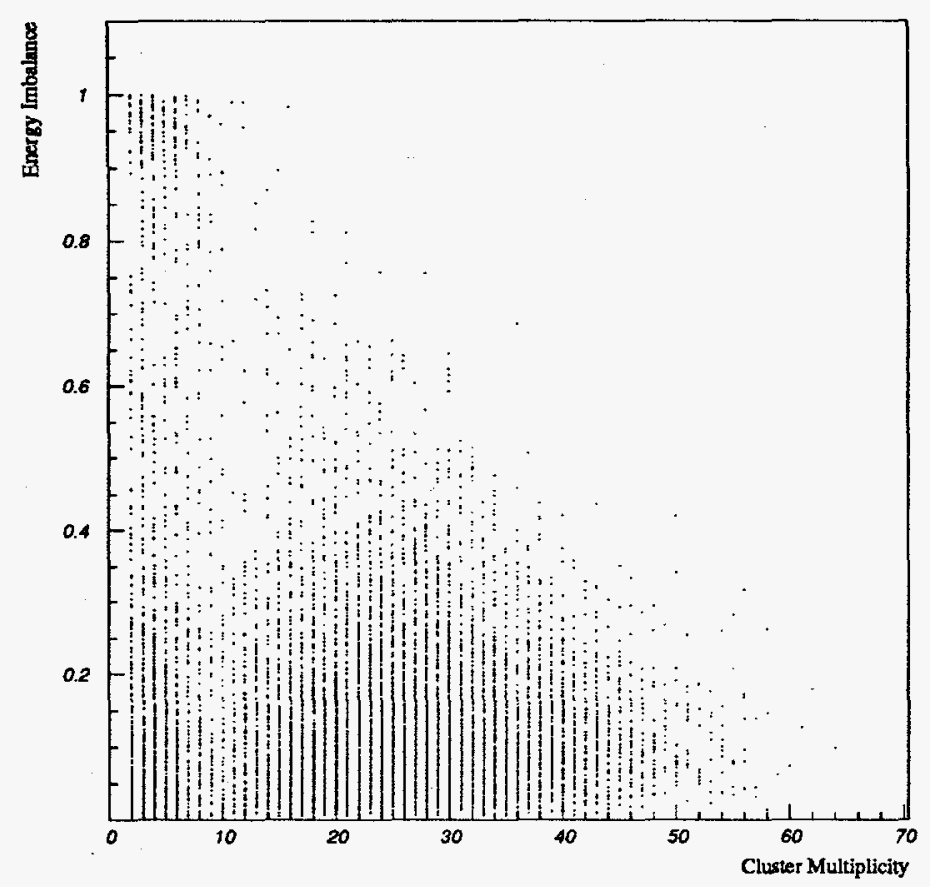

Figure 4-10: The Pass One data in $I$ versus $N_{c l u s}$ variable space.

Figures 4-11(A) and 4-11(B) show the normalized imbalance distributions for data with $21 \leq N_{\text {clus }} \leq 25$ and $12 \leq N_{\text {clus }} \leq 20$, respectively.

Assuming the background is primarily in the region $0.4<I<0.6$, then the expected signal fraction is estimated from figure 4-11(B) by the ratio of the number of events in the 0.4 $<I<0.6$ range $\left(N_{b, 1}\right)$ to the number of events in the $\mathrm{I}<0.4$ range $\left(N_{b, 2}\right)$. Let $N_{a, 1}$ and $N_{a, 2}$ be the numbers in the corresponding ranges in figure 4-11(A). The estimated number of backgrounds and resulting background fraction $\left(f_{b}{ }^{B}\right)$ is now

$$
\begin{gathered}
N_{b}^{B}=N_{a, 1}-N_{b, 2}\left(\frac{N_{a, 1}}{N_{b, 2}}\right)=58.1 \\
f_{b}^{B}=(0.12 \pm 0.08) \%
\end{gathered}
$$



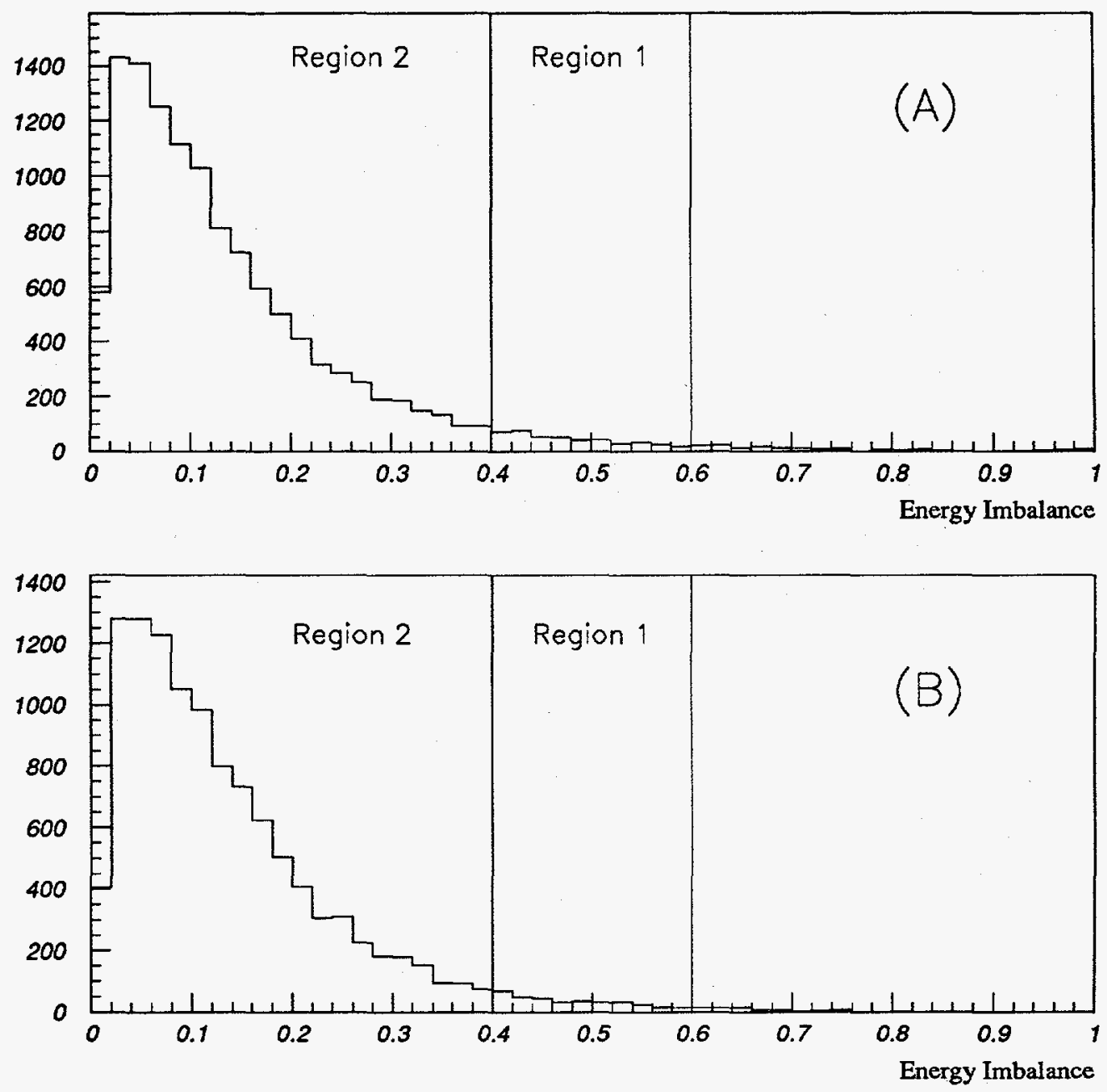

Figure 4-11: Imbalance distributions:
(A) $12 \leq N_{\text {clus }} \leq 20$
(B) $21 \leq N_{\text {clus }} \leq 25$ 
where the error primarily reflects the systematic uncertainty associated with this method of determination, in addition to the statistical error on a small number of selected events.

\subsubsection{Background Summary}

The background estimates from each source discussed above are summarized in table 4-3. The total fractional background, $f_{b}$, is estimated to be $(0.22 \pm 0.10) \%$.

\begin{tabular}{|c|c|c|c|}
\hline Source & $\begin{array}{c}\text { Number } \\
N\end{array}$ & $\begin{array}{c}\text { Fraction of total } \\
\text { background }\end{array}$ & $\begin{array}{c}f_{b} \\
(\%)\end{array}$ \\
\hline \hline Bhabha & 51 & $0.45 \pm 0.27$ & $0.10 \pm 0.06$ \\
\hline Cosmic Ray & 2.5 & 0.02 & $<0.005$ \\
\hline $2 \gamma$ & 1.5 & 0.01 & $<0.003$ \\
\hline$\gamma$ & negligible & 0 & 0 \\
\hline Beam-related & 58 & $0.52 \pm 0.35$ & $0.12 \pm 0.08$ \\
\hline \hline Totals: & 113 & 1.00 & $0.22 \pm 0.10$ \\
\hline
\end{tabular}

Table 4-3: Summary of 1993 background estimate.

The fraction of total background for Bhabhas given in table 4-3 is a number required for the calculation of the background asymmetry.

\subsection{The Background Asymmetry}

The asymmetry of the background is due entirely to the Bhabha content. It would be dangerous, however, to assume that the background Bhabhas have the same angular distribution as the theoretical distribution. Therefore we base our asymmetry estimate on a convolution of the angular distribution of our identified Bhabhas (see section 4.4.1) with the theoretical asymmetry distribution. The experimental angular distribution is shown in figure 4-12.

The theoretical asymmetry distribution is calculated in two steps. First we use the Alibaba Monte Carlo program [67] to predict the relative contributions of the $Z$ and photon exchanges to the total cross section as a function of angle. Then the measured asymmetry 


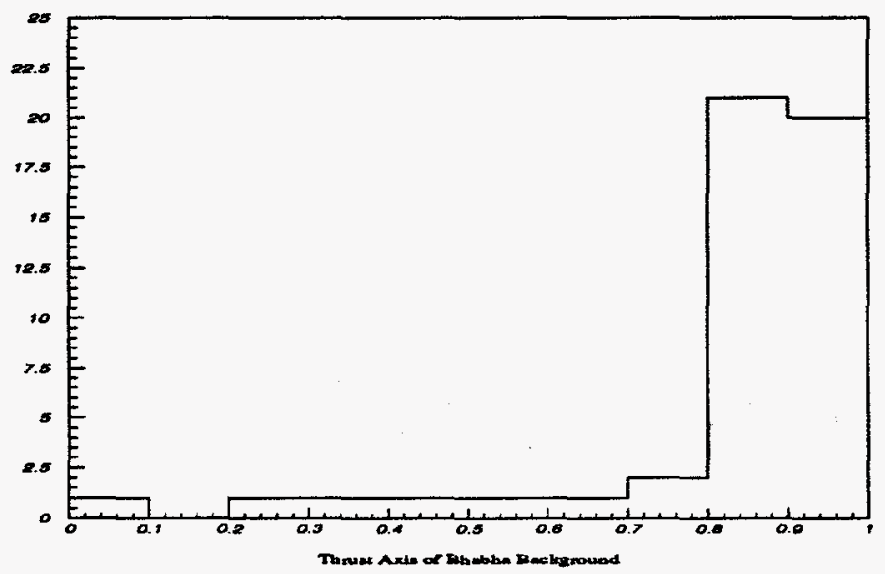

Figure 4-12: The angular distribution of the identified Bhabha sample.

[equation (4.2)] is assumed for the $Z$ contribution, and diluted by the photon contribution. The resulting Bhabha asymmetry integrated over polar angle is

$$
A_{\text {Bhabha }}=0.046 \pm 0.010
$$

which must be combined with the Bhabha background fraction (see table 4-3) to find the background asymmetry $A_{b}$ :

$$
A_{b}=(0.45 \pm 0.27) \cdot(0.046 \pm 0.010)=0.021 \pm 0.013
$$

This number, along with the total background fraction, is used to correct the measured asymmetry $A_{N}$ in the next chapter. 


\section{Chapter 5}

\section{Analysis and Results}

It was shown in Chapter 1 how the measured asymmetry $A_{N}$ is related to $A_{L R}$. It was assumed that there no other asymmetries that might contaminate the measurement. An additional implicit assumption was that the average Compton measured asymmetry, $\left\langle P_{e}^{C}\right\rangle$, is equal to the true luminosity-weighted polarization at the SLC interaction point, $\left\langle P_{e}\right\rangle$. In turns out that in 1993 this may not have been the case. Both of these assumptions will be addressed in this chapter, and some systematic cross-checks which have been made over the last two years will also be discussed, after which the final result will be presented.

\subsection{The Chromaticity Effect}

Because the SLC was run in flat-beam mode, with very small vertical emittance, the vertical size of the beam at the IP may have been limited by 3rd order chromatic aberrations. This is to say, that the focussing may have been energy-dependent. As a result, the luminosity may also have been energy dependent. As discussed in section 2.2, the spin orientation is certainly energy dependent. Therefore there could be a luminosity-polarization correlation where the lower energy tail of the beam has lower polarization and also does not contribute significantly toward the creation of $Z$ s. This implies that the instanta- 
neous polarization at the SLC interaction point, $P_{e}$, would be a weighted-average over number density and luminosity

$$
P_{e}=\frac{\int N(E) P(E) L(E) d E}{\int N(E) L(E) d E}
$$

where $N$ is the number density of electrons, $P$ is the polarization, and $L$ the luminosity, all functions of the energy $E$.

At the Compton polarimeter interaction point, on the other hand, the electron beam has zero nominal dispersion. Also, the Compton target laser spot is large compared to the electron beam, and as a result the polarimeter measures the polarization of the entire beam profile:

$$
P_{e}^{C}=\frac{\int N(E) P(E) d E}{\int N(E) d E}
$$

Therefore there can be a difference between $\left\langle P_{e}^{C}\right\rangle$ and $\left\langle P_{e}\right\rangle$ that should be taken into account, a difference encompassed in the parameter $\xi$, which we call the chromaticity correction.

$$
\left\langle P_{e}\right\rangle=(1+\xi)\left\langle P_{e}^{C}\right\rangle
$$

Although the chromaticity effect may vary over time during the run, only the average luminosity-weighted polarization is important to the analysis, and thus a single correction parameter is sufficient.

\subsubsection{The Chromaticity Model}

The various inputs necessary to calculate $\xi$ are shown in figure 5-1. The first is the intensity $(N)$ versus energy profile, which is measured by wire scans. The second is the 
energy dependence of the spot size; what is shown is a calculation based on simple accelerator modeling. The luminosity is then given by

$$
L(E)=\frac{N_{e^{-(E)} N_{e^{+}}(E)}}{4 \pi \sigma_{x}(E) \sigma_{y}(E)}
$$

where $N$ is the number density for the subscripted current, and $\sigma$ is the RMS size of the beam in the subscripted dimension.

The third input to the model is the polarization versus energy dependence which was measured directly (see section 2.1.4). The model predicts that $\xi$ is very small $(<0.002)$ for the Gaussian core of the beam $(\Delta E / E \approx 0.2 \%)$. However, as figure 5-1(a) shows, $N(E)$ has a low energy tail extending to $\Delta E / E=1 \%$, at which energy electrons have significantly lower polarization, but are less focused. The complete chromaticity correction is estimated to be $\xi=0.019 \pm 0.005[68]$.

However, for the correction of the observed Compton polarization, we prefer to employ a conservative and essentially model-independent estimate which is based on experimental observations. The chromaticity correction is rigorously limited by the following relation:

$$
(1+\xi) \leq\left(\frac{P_{e}}{P_{e}^{l i n a c}}\right)_{\max } \cdot\left(\frac{P_{e}^{\text {linac }}}{P_{e}^{C}}\right)_{\max }
$$

where $P_{e}^{\text {linac }}$ is the polarization at the end of the linac, and the other polarizations are defined in equations (5.1) and (5.2). We determine an upper limit on $\xi$ by finding the upper limits of the two polarization ratios defined in this equation.

To compare $P_{e}^{\text {linac }}$ and $P_{e}{ }^{C}$ we may rely on direct measurements. On July 2 , a special test was used to directly measure $P_{e}^{\text {linac }}$. Keep in mind that there is only one major effect which serves to make $P_{e}{ }^{C}$ less than $P_{e}$ linac and that is spin diffusion, particularly that arising from the low energy tail. For this experiment, the SLC was run in a special low current mode, in which the core width of the energy distribution was narrowed $(\leq 0.1 \%)$ and the low energy tail was removed. Under these conditions, the Compton polarimeter effectively measures the polarization at the end of the linac. 


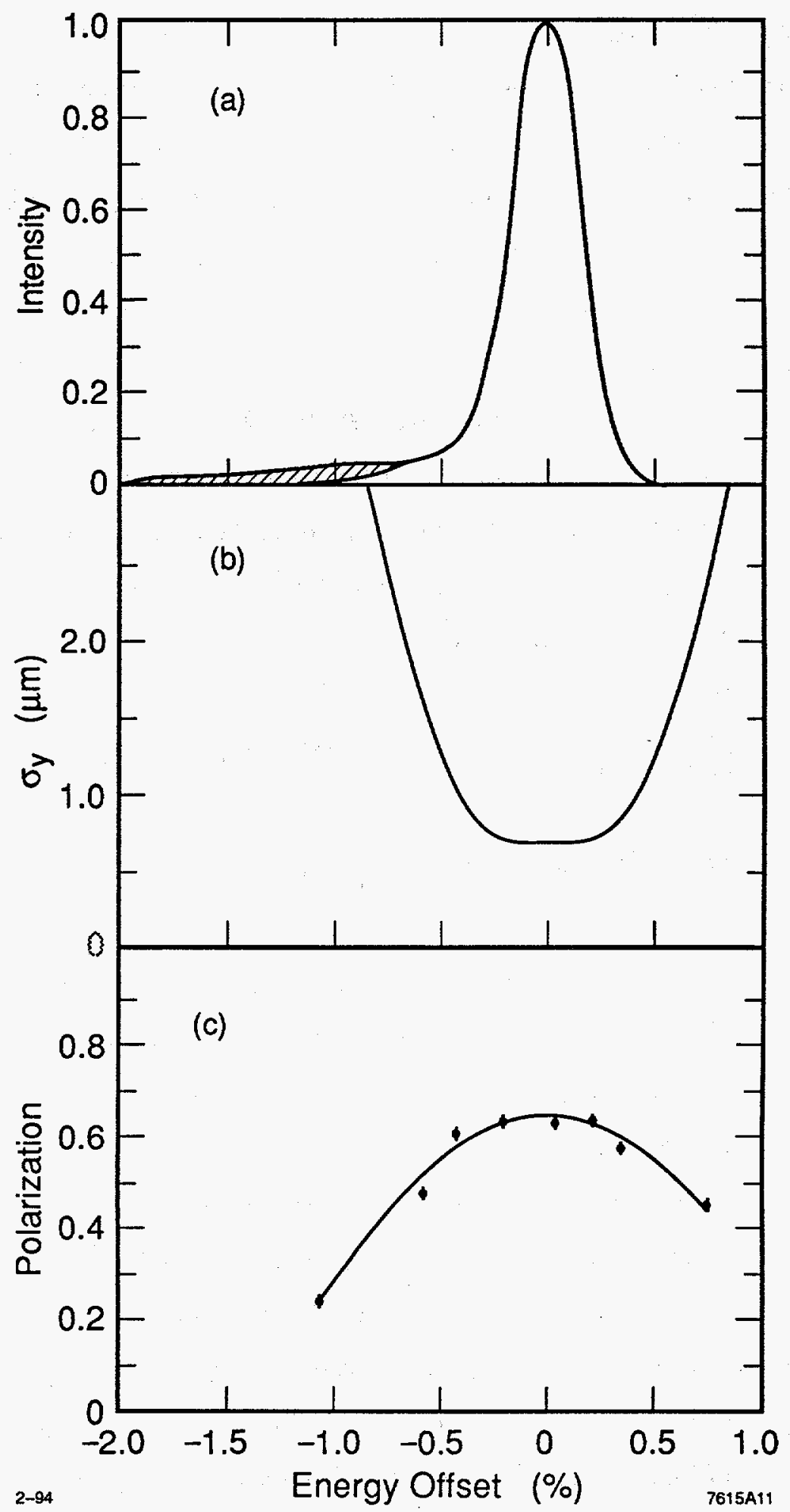

Figure 5-1: (a) $N_{e-}(E),\left(\right.$ b) $\sigma_{y}(E)$, and (c) $P_{e}(E)$ in the chromaticity model. 
The measurement was repeated on July 15th [37]. The combined results of these measurements gives $P_{e}^{\text {linac }}=(65.7 \pm 0.6) \%$, where the error is statistical only. Since we plan to take the ratio of two Compton measured polarizations, systematic errors cancel.

If we refer to the linac measurements made with the diagnostic Møller polarimeter, recorded after the source laser wavelength was optimized at $865 \mathrm{~nm}$, we find that the linac polarization is consistent with a constant number. Also the relative stability of the Compton measured polarization (see figure 3-17) indicates that the source polarization was stable in time. This is not entirely the case, of course; for instance, we know there are variations in source polarization when the cathode is freshly cesitated. But for the estimate of $P_{e}^{\text {linac }} / P_{e}^{C}$ we assume that the linac polarization is constant and the variation in the Compton measured polarization is primarily a function of the energy collimation of the electron beam.

The luminosity-weighted average Compton polarization for the post-PLS-wavelength change is $(63.15 \pm 0.01) \%$. The ratio is then

$$
\frac{P_{e}^{l i n a c}}{P_{e}^{C}}=\frac{(65.7 \pm 0.6)}{(63.15 \pm 0.01)}=1.0438 \pm 0.0095<1.0628(95 \% \text { c.1. })
$$

where we approximate the $95 \%$ confidence limit by the two standard-deviation upper limit on the ratio.

It is desirable to test the hypothesis that the fluctuations in Compton polarization are indeed related to the energy collimation of the electron beam. Fortunately, a careful study of the correlation of polarization with SLC machine parameters has already been carried out [69]. It was discovered that the position of the SL-3 collimator, known to intercept the low energy tail of the electron beam, is correlated with the measured Compton polarization. Figure 5-2 shows the Compton polarization versus the difference between the SL-3 collimator position and the beam offset, measured by a beam profile monitor, for data taken after the source laser wavelength change (it is assumed that the source polarization and hence the linac polarization is stable after this point in time). A strong correlation is evident over the ranges of collimator settings shown. As the relative collimator position becomes more negative, more of the beam tail is occluded, and the measured Compton polarization 
increases, as expected, since the off-energy and low polarization components of the beam are removed.

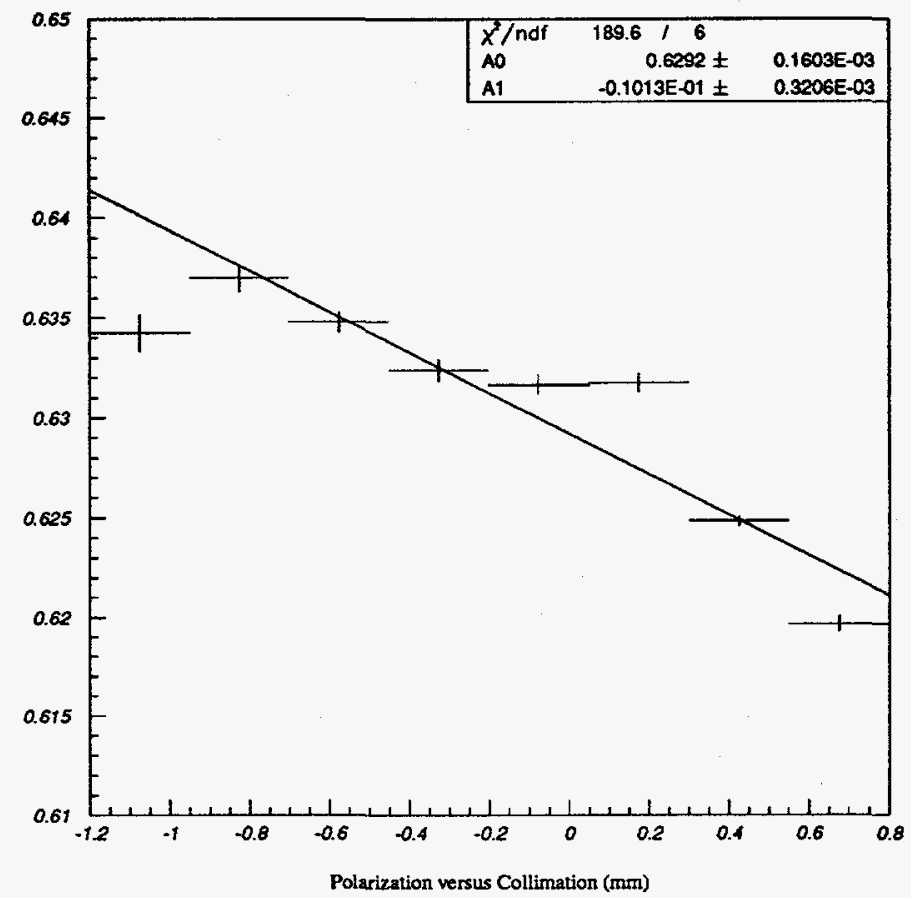

Figure 5-2: The position of the SL-3 collimator vs. the measured Compton polarization.

Now we must estimate the ratio of the luminosity-weighted IP polarization to the linac polarization. Chromaticity effects aside, $P_{e}$ must be less than $P_{e}^{\text {linac }}$, because of spin diffusion in the arc. Therefore the ratio is bounded by 1.0 , and we must look for effects that maximize $P_{e}$. This means that the most conservative estimate is one which ignores the energy tail of the beam and assumes that all of the polarization comes from the core of the $P_{e}(E)$ distribution. The upper bound on this ratio is determined by a TURTLE transport simulation of the arc and final focus region, with the very conservative (that is, tend to maximize the ratio) beam parameters listed in table 5-1. This simulation predicts an upper limit of 0.986 on the ratio of IP polarization to linac polarization [70]. 


\begin{tabular}{|c|c|}
\hline Parameter & Assumed Limit \\
\hline \hline$\theta_{y}^{r m s}$ & $<200 \mu \mathrm{rad}$ \\
\hline$\theta_{x}^{r m s}$ & $<350 \mu \mathrm{rad}$ \\
\hline$\varepsilon_{y}$ & $>650 \mu \mathrm{m}-\mu \mathrm{rad}$ \\
\hline$\varepsilon_{x}$ & $>100 \mu \mathrm{m}-\mu \mathrm{rad}$ \\
\hline$\sigma_{E}$ & $>0.15 \%$ \\
\hline \hline$P_{e} / P_{e}^{\text {linac }}$ & $<0.986$ \\
\hline
\end{tabular}

Table 5-1: Parameters of beam transport simulation.

The final limit on the chromaticity corrections is

$$
\begin{gathered}
1 \leq(1+\xi) \leq(1.0628 \cdot 0.986) \\
\text { or } \\
0 \leq \xi \leq 0.048
\end{gathered}
$$

We take the central value of the allowed range as the correction, and the $62.8 \%$ width of the allowed range as the error (since the correction has a more or less equal probability of lying anywhere in the allowed range) to find

$$
\xi=0.024 \pm 0.016 .
$$

Figure 5-3 shows the predictions of the full accelerator model developed by F. Decker

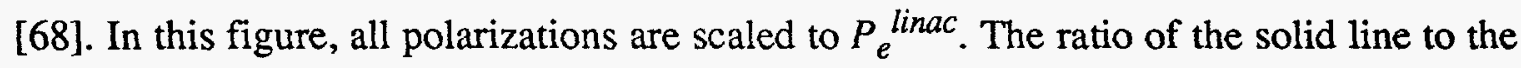
dashed line (for a given year) is equal to $(1+\xi)$. It was decided by the collaboration not to use this model as the correction because many inputs remain untested by data. The point of interest is that, in 1994, the chromaticity effect should be negligible, for two reasons. First, an upgrade of the final focus optics will reduce the chromatic aberrations. And second, the so-called "over-compression" technique will be used in the damping ring to remove the low-energy tail of the electron bunch (the amount of energy-tail is represented by the degree to which the dotted lines deviate from 100\%). 


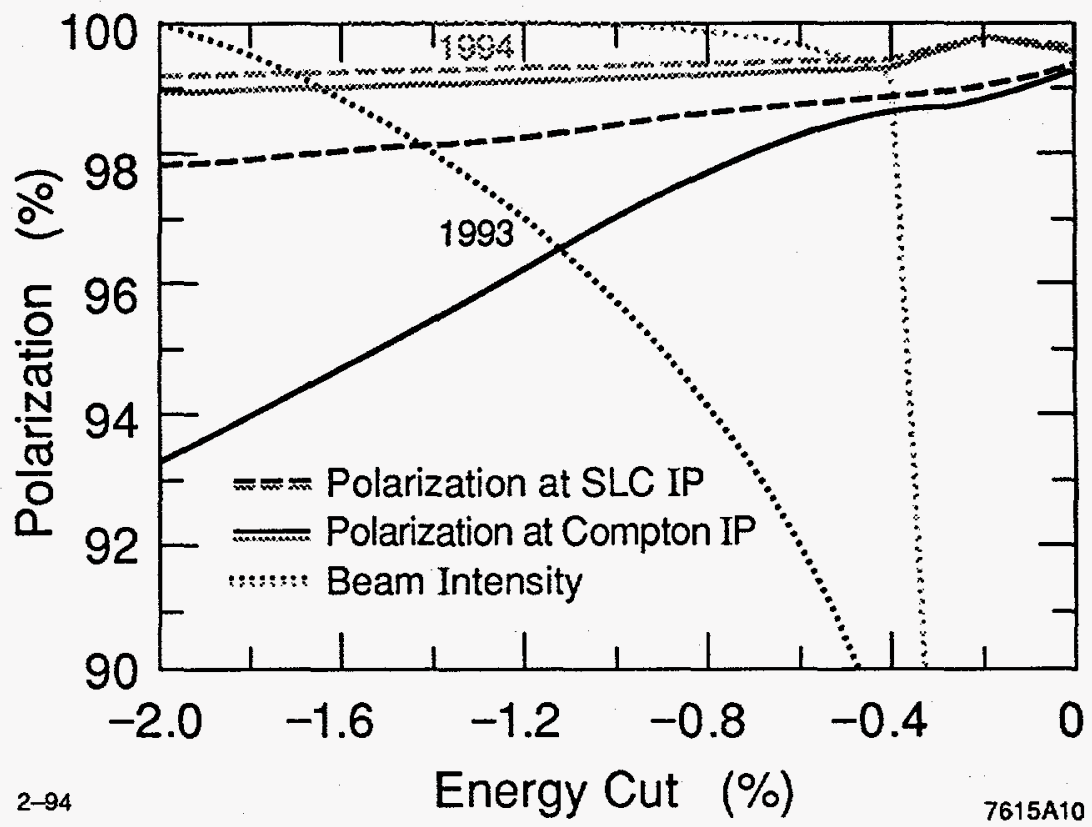

Figure 5-3: Predictions of the chromaticity model.

\subsection{Additional Corrections to $A_{L R}$}

The following equation shows the first order effect of various asymmetries on $A_{L R}$ :

$$
\begin{aligned}
A_{L R}=\frac{A_{N}}{\left\langle P_{e}\right\rangle}+\frac{1}{\left\langle P_{e}\right\rangle}[ & f_{b}\left(A_{N}-A_{b}\right)-A_{L}-A_{N}^{2} A_{P} \\
& \left.-E_{c m} \frac{\sigma^{\prime}\left(E_{c m}\right)}{\sigma\left(E_{c m}\right)} A_{E}-A_{\varepsilon}+\left\langle P_{e}\right\rangle P_{P}\right]
\end{aligned}
$$

In this equation, $A_{N}$ is the measured asymmetry defined in equation (1.29), $\left\langle P_{e}\right\rangle$ is the luminosity-weighted average polarization defined in equation $(1.30), f_{b}$ is the background fraction in the sample, and $A_{b}$ is the asymmetry of that background, as discussed in section 4.5. The polarization asymmetry, $A_{p}$, is directly measured by the polarimeter, as discussed in section 3.3.1. Other terms in equation (5.9) include the luminosity asymmetry $\left(A_{L}\right)$, the center-of-mass energy $\left(E_{c m}\right)$ and the energy asymmetry $\left(A_{E}\right)$, the cross section $(\sigma)$ and the derivative of the cross section $\left(\sigma^{\prime}\right)$ at the center-of-mass energy, the efficiency asymmetry $\left(A_{\varepsilon}\right)$, and the positron polarization $\left(P_{P}\right)$, which are discussed in the sub-sections below. 


\subsubsection{Luminosity Asymmetry $\left(A_{L}\right)$}

It has been shown empirically that the current extracted from strained cathodes varies with the orientation of linearly polarized light with respect to the strain axis. Therefore residual linear polarization in the source laser could result in an asymmetry in the extracted electron current, which in turn leads to a net left-right luminosity asymmetry $A_{L}$. This luminosity asymmetry is estimated through the $120 \mathrm{~Hz}$ data of beam current toroids and radiative Bhabha monitors located in the North Final Focus region. We find the asymmetry to be $A_{L}=(3.8 \pm 5.0) \times 10^{-5}$ which leads to a correction of $(-0.037 \pm 0.049) \%$ to $A_{L R}$ [71].

The beam current asymmetry and $A_{Q}$ of all other quantities correlated with it were reduced by once reversing the sign of the field in the LTR solenoid. By changing the sign of the field in the LTR solenoid, electrons that were previously stored with spins up in the damping ring are now stored down. Those same electrons now have the opposite helicity at the IP. In other words, the meaning of the source helicity bits has changed, and the sign of any source-related asymmetry also changes.

A cross check on the luminosity asymmetry is performed by examining a sample of 125,375 small-angle Bhabha events measured by the LUM system. The observed left-right counting asymmetry, $A_{N}$, for small angle Bhabha events is very small $\left(\approx-1.5 \times 10^{-4} \cdot P_{e}\right.$ for the LUM acceptance), and therefore this quantity is a direct measure of $A_{L}$. The measured value of $A_{N}{ }^{L U M}=(-32 \pm 28) \times 10^{-4}$ is consistent with the more precisely determined value of the luminosity asymmetry.

\subsubsection{Energy Asymmetry $\left(A_{E}\right)$}

In equation (5.9), $\sigma$ and $\sigma$ ' are the cross section and derivative of the cross section with respect to energy, respectively, evaluated at the average operating energy. The energy asymmetry is measured directly by the energy spectrometer (see section 2.4$) 120 \mathrm{~Hz}$ data to be $(4.4 \pm 0.1) \times 10^{-7}$. This asymmetry follows the beam current asymmetry due to beam loading in the accelerator. At the measured value of $91.26 \mathrm{GeV}$ the fractional derivative of the cross section with respect to energy is not terribly steep $\left(0.023 \mathrm{GeV}^{-1}\right)$. Thus the fractional correction to $A_{L R}$ of $(0.00090 \pm 0.00002) \%$ is negligible. 


\subsubsection{Efficiency Asymmetry $\left(A_{\varepsilon}\right)$}

The detector itself could induce an false asymmetry by preferentially accepting Zs with one handedness. The only difference between left-handed and right-handed $Z s$ is that the polar-angular distribution of outgoing fermions is reversed; or, to put it another way, the distribution of fermions for a left-handed event is the same as the distribution of anti-fermions in a right-handed events. Therefore the only way to have a non-zero $A_{\mathcal{\varepsilon}}$ is if fermions and anti-fermions are not accepted equally in the calorimeter.

First of all, it is assumed that the process of calorimetry itself is symmetric with respect to matter and anti-matter. Given this assumption, it is sufficient to have either an azimuthally symmetric magnetic field or uniform detection efficiency for a given polar angle in order for the determination of $A_{L R}$ to be independent of efficiency effects. In the case of the SLD, both of these conditions are true: The LAC is highly uniform and symmetric (both in $\theta$ and $\phi$ ) in its construction, and the SLD solenoidal field implies magnetic azimuthal symmetry.

Even without the assumption of matter/anti-matter detection efficiency, it is sufficient that the efficiency be polar-angle symmetric, that is, that $\varepsilon(q, \theta)=\varepsilon(q, \pi-\theta)$, which is also a symmetry of the SLD construction. Even if there were some non-uniformity in the calorimeter energy response, beyond the symmetries already described, our energy cut-off is so low (see figure 4-4) that such a bias would have a negligible effect. For all of these reasons, the $A_{\varepsilon}$ correction term is assumed to be negligible.

\subsubsection{Positron Polarization $\left(P_{P}\right)$}

It has already been discussed in section 2.1.1 that any residual polarization of positrons is correlated with the scavenger electron polarization and has no effect on $A_{L R}$. Therefore, in order for there to be an effect, the positrons must have some fixed-sign polarization building up somewhere. The only known mechanism for the build-up of fixed-sign positron polarization is the Sokolov-Turnov effect: the induced quantum spin-flip transitions in the magnetic field of the positron damping ring. 
The polarization as a function of time in a storage ring is given by [72]

$$
\begin{aligned}
P(t) & =\frac{8}{5 \sqrt{3}}\left(1-e^{-t / \tau}\right) \\
\tau & =\frac{4 m^{2} c^{2} R^{3}}{5 \sqrt{3} \pi h e^{2} \gamma^{5}}
\end{aligned}
$$

where $t$ is the storage time in the ring, $m$ is the mass of the stored particle (in this case the electron mass), $\gamma$ is the Lorentz factor, and $R$ is the radius of the ring. This effect is limited by the ratio of the storage time ( $t=16.6 \mathrm{~ms})$ to the polarizing time ( $\tau=960 \mathrm{~s})$, which is to say that the maximum polarization of a positron bunch is $\leq 1.6 \times 10^{-5}$.

\subsubsection{Summary of Small Corrections}

A summary of the small corrections to $A_{L R}$ discussed in this section is presented in table 5-2.

\begin{tabular}{|c|c|c|c|}
\hline Correction & Parameter & Value $\left(10^{-4}\right)$ & $\Delta A_{L R} / A_{L R}(\%)$ \\
\hline \hline $\begin{array}{c}\text { Background fraction } \\
\text { Background Asymmetry }\end{array}$ & $\mathrm{f}_{\mathrm{b}}$ & $22 \pm 10$ & $+0.18 \pm 0.09$ \\
& $\mathrm{~A}_{\mathrm{b}}$ & $210 \pm 130$ & \\
\hline Efficiency Asymmetry & $\mathrm{A}_{\varepsilon}$ & $\approx 0$ & $\approx 0$ \\
\hline Luminosity Asymmetry & $\mathrm{A}_{\mathrm{L}}$ & $0.38 \pm 0.50$ & $-0.037 \pm 0.049$ \\
\hline Polarization Asymmetry & $\mathrm{A}_{\mathrm{P}}$ & $-33 \pm 1$ & $-0.034 \pm 0.001$ \\
\hline Energy Asymmetry & $\mathrm{A}_{\mathrm{E}}$ & 0.0044 & $0.00090 \pm 0.00002$ \\
\hline Positron Polarization & $\mathrm{P}_{\mathrm{p}}$ & $<0.16$ & $<0.010$ \\
\hline \hline Total Correction & & & $0.12 \pm 0.10$ \\
\hline
\end{tabular}

Table 5-2: Summary of corrections to $A_{L R}$.

The total correction applied to $A_{L R}$ is $0.12 \%$, with a systematic error of the same order. This correction is negligible compared to the chromaticity correction and the uncertainty on the polarization measurement. 


\subsection{Systematic Cross-checks}

In addition to the systematic effects discussed above, there are several "non-effects" to be considered, that is, effects that could conceivably affect the measurement but have been shown not to do so.

\subsubsection{Fixed Polarizer Test}

The cross-checks discussed in this section and the section following were motivated by the one-time discrepancy between the Linac Møller Polarimeter and Compton polarization measurements, which was on the order of $10 \%$. Thus these checks were designed to look for a large systematic error of essentially unknown origin, unlike the precision checks on polarimetry discussed in Chapter 3.

It is possible that the circular polarization delivered by the CLS transport line is less than the reported 99\%, that somehow the Pockels cell scans are only finding a local maximum rather than the absolute maximum circular polarization. In order to discount this possibility, a special test (called the "fixed polarizer test") was prepared [73].

In this test, the circularly polarizing Pockels cells were removed, and linearly polarized light was transmitted to the SLC vacuum entrance window. At this point a quarter wave plate was introduced, and the circular polarization of the beam immediately before the entrance window was measured to be $0.995 \pm 0.005$. Then nominal beam polarization measurements were made (with fixed photon helicity), and the results of those measurements are shown in figure 5-4.

The average beam polarization measured before the quarter-wave plate was installed was $0.607 \pm 0.004$. The average beam polarization measured after the quarter-wave plate was installed was $0.601 \pm 0.005$. Thus the Pockels cell polarization technique is eliminated as a possible source of large unknown systematic error.

\subsubsection{Current Asymmetry Test}

Having eliminated the target polarization as a source of large unknown systematic error, the only remaining possibility was in the measurement of the experimental asymme- 


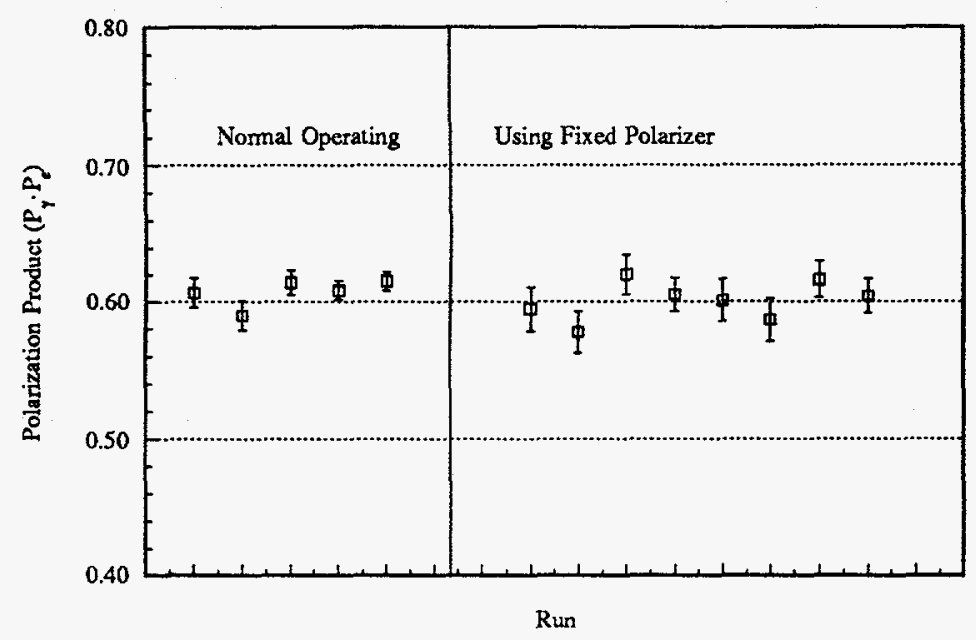

Figure 5-4: The fixed-polarizer test.

try itself. In order to test the polarimeter data acquisition and analysis, an experiment was conceived wherein a left-right current asymmetry is induced in an unpolarized electron beam. The polarimeter then measures the resulting Compton luminosity asymmetry and compares with the current asymmetry measured by a beam toroid [74].

First the PLS Pockels cell was set to its half-wave voltage, resulting in linearly polarized light illuminating the photocathode and extracting unpolarized electron bunches. By adding a linear polarizer to the PLS optics, immediately after the Pockels cell, the cell could act as a variable attenuator. A L/R current asymmetry was introduced by asymmetrically setting the $L / R$ voltages of the Pockels cell, which means that the cathode was illuminated with different intensities on left and right pulses. The LTR solenoid in the damping ring extraction line was then turned off in order to insure the randomization of the electron bunch polarization, which was then measured by the Compton polarimeter to be consistent with zero.

The induced current asymmetry was measured by the polarimeter and compared against measurements of the FF11-1275 beam toroid. The result of the test is shown in figure 5-5. 
The polarimeter is demonstrated to measure the correct current asymmetry to better than $3 \%$, so again, a large unknown systematic effect is ruled out.

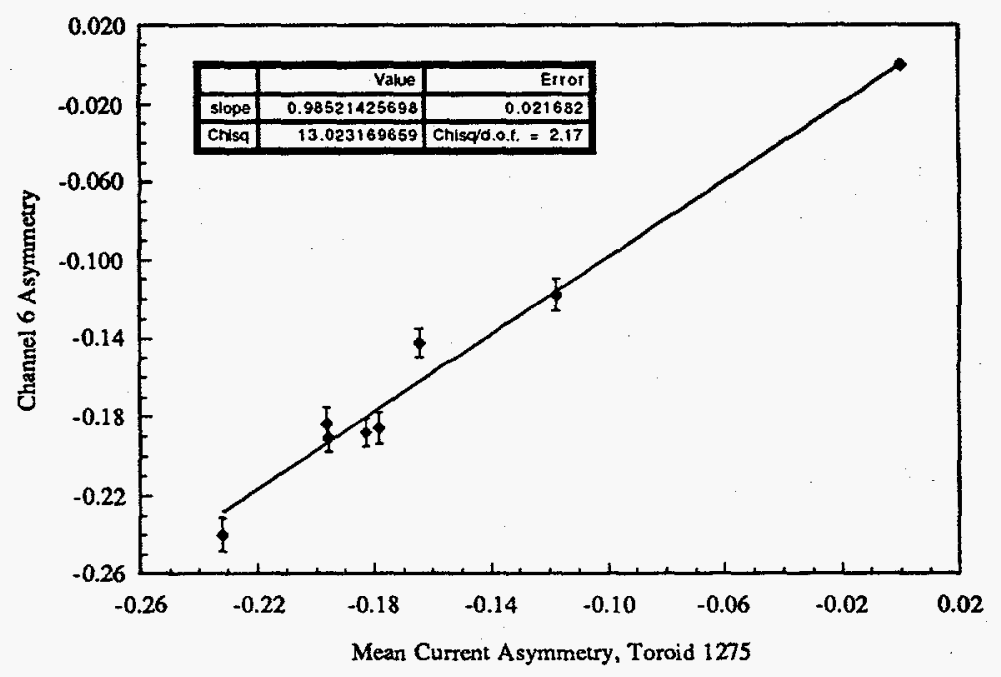

Figure 5-5: The induced current asymmetry test.

\subsubsection{IP-Compton Spin Precession}

Aside from the chromaticity effect, there are two other possibilities that could make the Compton measured polarization differ from the IP polarization. One is depolarization caused by beam-beam interactions. This effect is expected to be small [75]. Unfortunately, it is not possible to measure this effect directly through raw polarimeter data, because when the positron beam drops out, the beam loading effect in the accelerator changes the energy (and hence longitudinal polarization) of the electron beam. Summed polarimeter data is protected from this second effect by the positron current veto.

Another possibility is simple spin-precession. Although there are no dipole fields between the IP and the CIP, there are quadrupole magnets. However, the total beam deflection caused by the quadrupoles has been directly measured to be less than $100 \mu \mathrm{rad}$, which means the spin precession is less than $10 \mathrm{mrad}$. The maximum fractional effect on $P_{e}$ is less than $0.01 \%$, which is negligible compared to other polarization systematics. 


\subsubsection{Bit Integrity}

It is essential that the state of the PLS Pockels cell (which controls the electron beam helicity) for each beam crossing be properly incorporated into the SLD data stream. One potential source of error is an isolated incorrect bit within the data stream. There are two ways in which we are protected against this type of error.

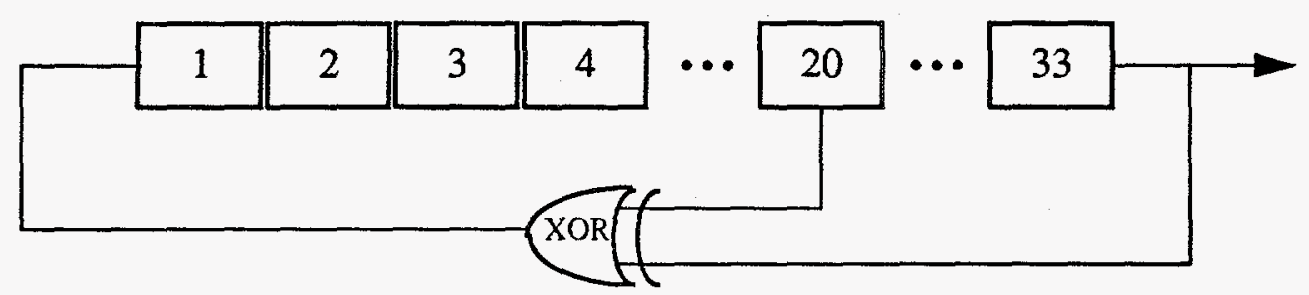

Figure 5-6: The PLS/CLS pseudo-random number generator.

The pseudo-random number generator used at the PLS and CLS is represented in figure 5-6 and has several important properties [76]. It is a 33-bit shift register which shifts every SLC pulse. The generator is unbiased; it generates an equal number of ones and zeros with no correlation between the output bit and any time-slot frequency. Any 33-bit sequence read by the SLD is sufficient to calculate every following bit for the entire sequence length $\left(2^{33}-1\right)$. Furthermore, the sequence is polarized. If a logical not were applied to the XOR output (equivalent to reversing the LIMO cable inputs of a register), the error could be detected within 34 beam crossings.

A real-time SLD VAX process uses this particular feature of the pseudo-random sequence to check of transmission reliability and flag errors [77]. This process counts and flags any pulse whose state is not predicted by the 33 bit seed sequence.

The integrity of the source bit transmission is further assured by the use of three redundant and independent transmission systems: the Klystron-veto-module (KVM) system, the direct transmission (Mach) line, and the PMON system.

A hierarchy determines which system is used to determine the sign of the $Z$ event. If the PMON helicity information is available and valid, then this information is assigned to the event record. Otherwise, if the Mach line data is available, it is assigned to the event record. 
The KVM is used only in the case that both PMON and Mach line data are missing, a condition which occurred during an early phase of the run, before PMON was installed.

In all the $Z$ data, the PMON bits were never observed to disagree with the Mach line bits. In sample of 500,000 triggers, the PMON disagreed with the MACH line only once, and in that case the PMON indicated an internal error state.

\subsubsection{Helicity Synchronization}

A different potential problem that is not detected by the bit sequence test or by the redundant system check is the possibility that the PLS bit sequence is not properly synchronized with the SLD data stream during some time period. This would cause all polarization information to be lost, and $A_{N}$ would be measured to be zero during such a period.

In 1992, two ingenious tests were devised to prove that the SLD data acquisition was synchronized with the PLS source bits [78]. The first is referred to as the beam dumper test. During this test, the beam was delivered only every 12 th pulse (that is, at $10 \mathrm{~Hz}$ ), and the PLS source bit pattern was set from random to RLLLLLLLLLLL. The right-handed pulse was then synchronized at the source with the $10 \mathrm{~Hz}$ beam delivery. Over the course of three runs, there were 6,146 events with ELO (defined in chapter 4) over $8 \mathrm{GeV}$, of which all were tagged ' $R$ '. This puts a limit on the synchronization error rate during the test at less than $0.05 \%$ at the $95 \%$ confidence level.

This test alone was not taken as conclusive. It was still possible (however unlikely) that the SLD data acquisition system could mix LAC and polarization data from different triggered events. In order to eliminate this possibility, a second experiment called the beam extinction test was devised. During this test, the beam helicity is chosen randomly, but the PLS optics are set extinguish left-handed pulses. Electrons only were delivered to the IP. The detector triggers were set to a low threshold, in order to trigger on beam-related radiation. Under these conditions, all triggered events should have had the right-handed helicity bit set, and these were called expected events. Events which had the left-handed helicity bit set were called unexpected events. 
The combined results of the beam extinction tests showed 5896 expected events with 9 unexpected events, which gives a conservative error rate of $(0.16 \pm 0.06) \%$, or less than $0.3 \%$ at the $95 \%$ confidence level. We call this conservative because it is probably a gross over-estimate: the unexpected events are almost certainly due to noise in the LAC firing the trigger and not an actual error in helicity assignment.

In early 1993 the beam extinction test was repeated and again confirmed that the SLD data acquisition was properly synchronized with the PLS helicity information.

\subsubsection{Cut Bias}

It would be alarming if the value of the measured asymmetry were somehow a function of the variables with which we select the data. Figure 5-7 shows the measured asymmetry in bins of the Pass Two cut variables. The solid line shows the value of $A_{N}$ on each graph. There are clearly no significant statistical anomalies in the energy and energy imbalance distributions.

At first sight, there appears to be a significant deviation of $A_{N}$ in the smallest bin of cluster multiplicity. We have carefully studied events in this bin. A hand scan of all events reveals nothing unusual in the event topologies. One might postulate that this bin could have a large number of Bhabhas that have just missed being removed by the multiplicity cut. However, the angular distribution of the events shows that they are mostly in the barrel region, the region in which Bhabhas do not have a significantly degraded asymmetry. Other beam backgrounds with null asymmetries are also unlikely to be concentrated in the barrel region. We are therefore forced to conclude that there is no excessive contamination in this particular $N_{c l u s}$ bin, and the observed deviation of $A_{N}$ is a two-sigma statistical fluctuation.

It is worthwhile to note that $A_{N}$ is also unbiased by the cut on the time difference between the $Z$ event and the polarization measurement. It can be further shown that, to the extent that the polarimeter randomly samples the true beam polarization, far fewer measurements than are made are actually necessary. This is again a direct consequence of the dependence of $A_{L R}$ on the average rather than the instantaneous polarization. Nevertheless, 

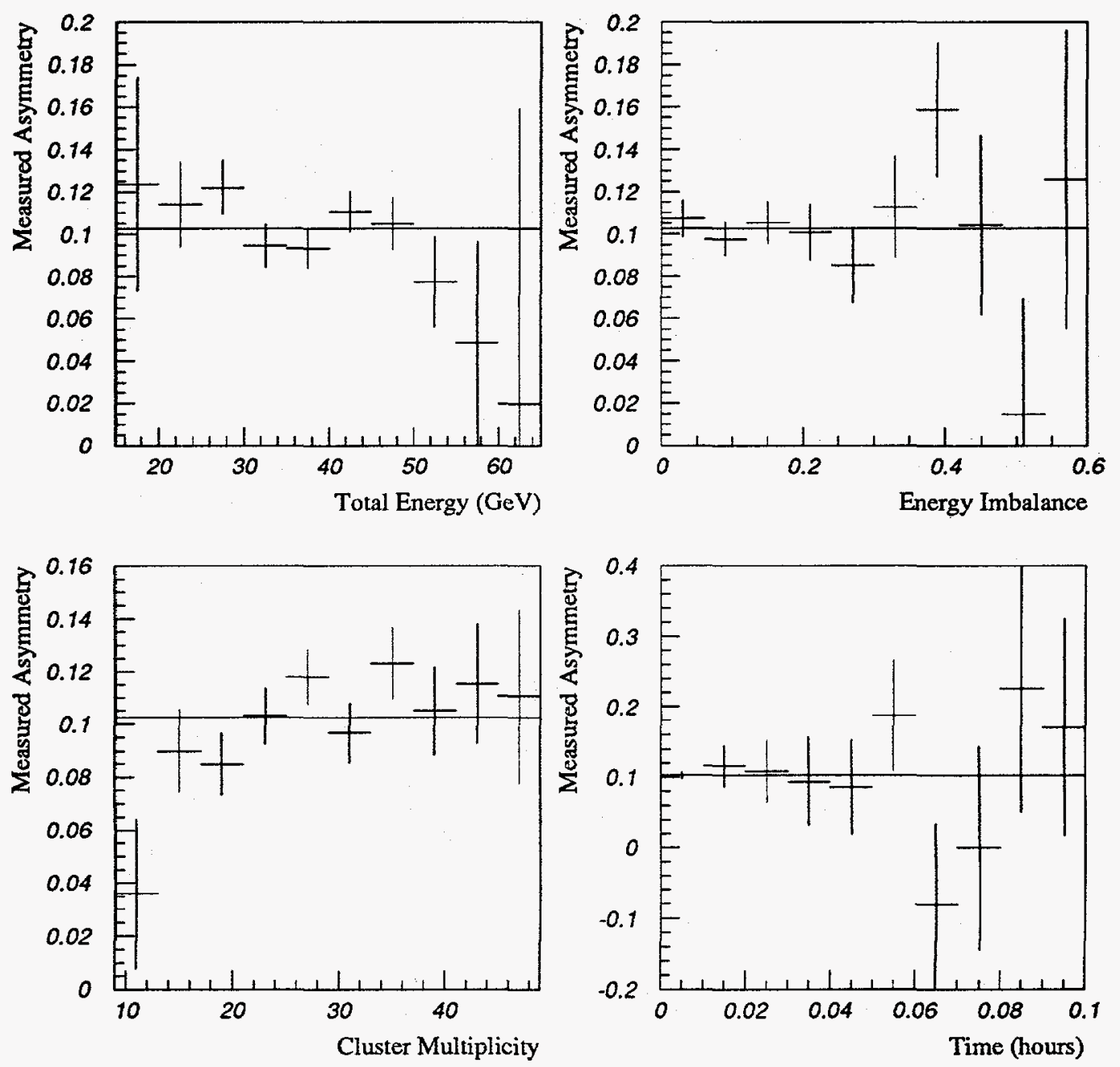

Figure 5-7: The measured asymmetry versus Pass Two cut variables. Solid lines indicate $A_{N}=0.1024$, the measured asymmetry of the entire $Z$ sample. 

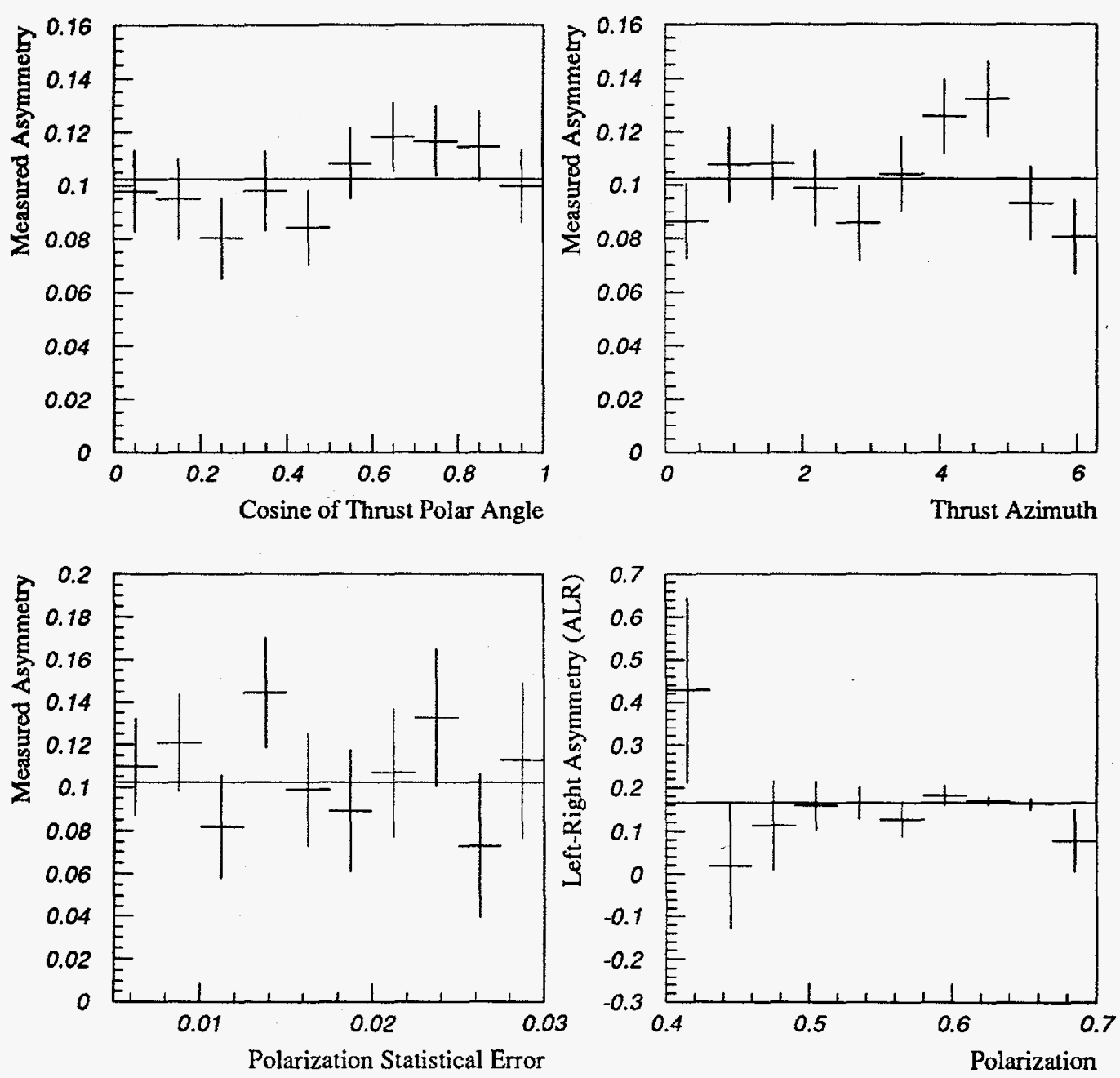

Figure 5-8: The measured asymmetry versus other event variables. 
in the unlikely case that the polarization did vary wildly during polarimeter down time, it remains safe to employ the time cut.

Figure 5-8 shows the measured asymmetry in bins of some other quantities of interest. The $\cos \theta$ and $\phi$ distributions indicate no unusual geometrical acceptance effects. There is no apparent correlation between $A_{N}$ and polarization error, which could appear if some background contamination were correlated with polarimeter noise and thus polarimeter statistical error. The value of $A_{L R}$ in polarization bins (that is, the measured asymmetry for events in the given range of polarization divided by the average polarization in that range) is also seen to be consistent with the average value for the run (the chromaticity correction has not been applied to the data shown).

\subsubsection{Total Systematic Uncertainty}

Table 5-3 shows the sum of all systematic uncertainties. Our total systematic error is 2.1\%. For the 1994 run, the chromaticity effect will be gone and the polarimeter error will be less than or equal to $1 \%$, so we expect our total systematic error will be $1 \%$ or less.

\begin{tabular}{|c|c|c|}
\hline Systematic Uncertainty & $\delta P_{e} / P_{e}(\%)$ & $\delta A_{L R} / A_{L R}(\%)$ \\
\hline \hline Laser Polarization & 1.0 & \\
\hline Detector Linearity & 0.6 & \\
\hline Interchannel Consistency & 0.5 & \\
\hline Analyzing Power Calibration & 0.4 & \\
\hline Electronic Noise Correction & 0.2 & \\
\hline \hline Total Polarimeter Uncertainty & 1.3 & 1.3 \\
\hline Chromaticity Correction $(\xi)$ & & 1.6 \\
\hline Asymmetry Corrections & & 0.1 \\
\hline \hline Total Systematic Uncertainty & & 2.1 \\
\hline
\end{tabular}

Table 5-3: Total systematic uncertainty on the $A_{L R}$ measurement. 


\subsection{Results and Comparisons}

We have now compiled sufficient information to extract $A_{L R}$ from the data. We will compare the result of this measurement with other electroweak measurements and the Standard Model. It is important to note, however, that $A_{L R}$ on its own makes no prediction about the Standard Model; only a comparison of at least two independent measurements can do so.

\subsubsection{Extracting $A_{L R}$}

From the results cited in chapter 4 , we find the measured asymmetry, $A_{N}$ :

$$
A_{N}=\frac{\left(N_{L}-N_{R}\right)}{\left(N_{L}+N_{R}\right)}=\frac{(27225-22167)}{(27225+22167)}=0.1024 \pm 0.0045
$$

where the error quoted is purely statistical.

From the results cited in Chapter 3, including the chromaticity correction derived in this chapter, we find the average polarization:

$$
\left\langle P_{e}\right\rangle=(1+\xi) \cdot \frac{1}{N_{Z_{i}}} \sum_{i}^{N_{z}}\left|P_{i}^{C}\right|=0.634 \pm 0.013
$$

where the error is purely systematic, a combination of the polarimeter systematic error and the chromaticity error.

Inserting these results and those of table 5-3 into equation (5.9) we determine the following result for $A_{L R}$ :

$$
A_{L R}(91.26 \mathrm{GeV})=0.1617 \pm 0.0071 \text { (stat.) } \pm 0.0033 \text { (syst.) }
$$

\subsubsection{Extracting the Weinberg angle from $A_{L R}$}

In order to compare to other electroweak experiments, we would like to convert our energy-dependent value of $A_{L R}$ into an observation of the effective Weinberg angle, $\sin ^{2} \theta_{W}^{\text {eff }}$. We use the ZFITTER program [24] to model initial-state radiation, and to account for the photon exchange and electroweak interference that occurs as a result of being off 


\begin{tabular}{|c|r|}
\hline Source of Correction & $A_{L R}=0.1617$ \\
\hline \hline$\sqrt{s}=91.26 \mathrm{GeV} \rightarrow \sqrt{s}=m_{Z}$ & -0.0015 \\
\hline$Z-\gamma$ interference & +0.0044 \\
\hline$\gamma$ exchange asymmetry dilution & +0.0003 \\
\hline weak box diagrams & negligible \\
\hline \hline Resulting value of $A_{L R}^{\circ}:$ & $=0.1649$ \\
\hline
\end{tabular}

Table 5-4: Converting the observed $A_{L R}$ into $A_{L R}^{o}$

the $Z$-pole energy, and convert our observed value of $A_{L R}$ into a value at the $Z$ pole. A summary of the contributions from these corrections is given in table 5-4. This calculation also agrees with results given by the EXPOSTAR program [79]. The result is

$$
A_{L R}^{o} \equiv \frac{2\left(1-4 \sin ^{2} \theta_{W}^{e f f}\right)}{1+\left(1-4 \sin ^{2} \theta_{W}^{e f f}\right)^{2}}=0.1649 \pm 0.0071 \text { (stat.) } \pm 0.0033 \text { (syst.) }
$$

We invert this relation and find the effective weak mixing angle to be

$$
\sin ^{2} \theta_{W}^{\text {eff }}=0.2292 \pm 0.0009 \text { (stat.) } \pm 0.0004 \text { (syst.). }
$$

When we combine this result with the 1992 SLD result [80], we find

$$
\begin{aligned}
\sin ^{2} \theta_{W}^{\mathrm{eff}} & =0.2295 \pm 0.0010 \\
A_{L R}^{o} & =0.1629 \pm 0.0078
\end{aligned}
$$

\subsubsection{Comparison with LEP data}

Figure 5-9 shows the SLD $\sin ^{2} \theta_{W}^{\text {eff }}$ along with the LEP collaboration measurements presented at the 1994 Moriond conference [8]. The SLD measurement is the most precise single measurement of this parameter. It also differs somewhat, by 2.2 standard deviations, from the average of the LEP measurements. 


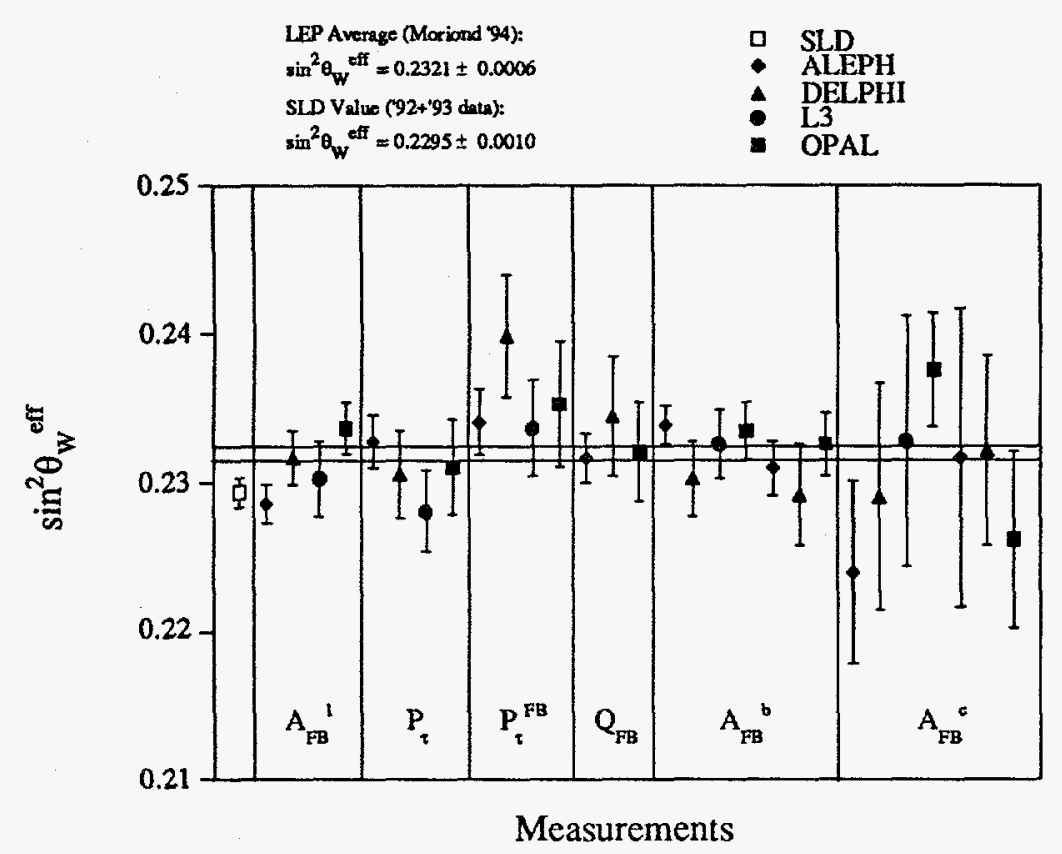

Figure 5-9: A comparison of LEP and SLD $\sin ^{2} \theta_{\mathrm{W}}{ }^{\text {eff }}$ measurements.

\subsubsection{Comparison with the Standard Model}

It is desirable to compare our results to those predicted by the Standard Model. In order to do this, we use a set of observables (excluding the SLD measurement) plus an assumed Higgs mass as inputs to ZFITTER, which then finds the best-fit top quark mass, and calculates the Standard Model prediction for $A_{L R}^{o}$. The results for various Higgs masses are presented in table 5-5 (predictions are presented with symmetrized errors). From this table of values we extract a prediction of $A_{L R}$ which is insensitive to the Higgs mass:

$$
A_{L R}^{o}(\text { Standard Model })=0.1409 \pm 0.0044
$$

which is over two standard deviations away from the SLD measurement [equation (5.16)].

Another way to compare the SLD result to the Standard Model is to use the S-T parameterization of Peskin and Takeuchi [17], described in chapter 1. Neutral current observables are independent of the $U$ parameter. It is therefore reasonable to plot the trajectories of observables in $S$ - $T$ space, and use such plots to draw conclusions about the oblique correc- 


\begin{tabular}{|c|c|c|}
\hline$m_{H}(\mathrm{GeV})$ & $m_{t}(\mathrm{GeV})$ & $A_{L R}^{o}$ \\
\hline \hline 60 & $149.3 \pm 12.0$ & $0.1424 \pm 0.0029$ \\
\hline 300 & $167.3 \pm 10.9$ & $0.1402 \pm 0.0029$ \\
\hline 1000 & $184.2 \pm 10.6$ & $0.1395 \pm 0.0030$ \\
\hline
\end{tabular}

Table 5-5: ZFITTER predictions for top mass and $A_{L R}$ at the $Z$ peak

tions implied by such measurements. Figure 5-10 shows the projection of three electroweak observables in $S-T$ space. The diamond shape in the middle of the graph delineates the effective shift in the $S$ and $T$ origin, relative to the reference point $\left(m_{t}=150 \mathrm{GeV}, m_{H}=\right.$ $1 \mathrm{TeV}$ ), for the range of top and Higgs masses noted. The range of top mass is chosen in light of the recent CDF measurement [9].

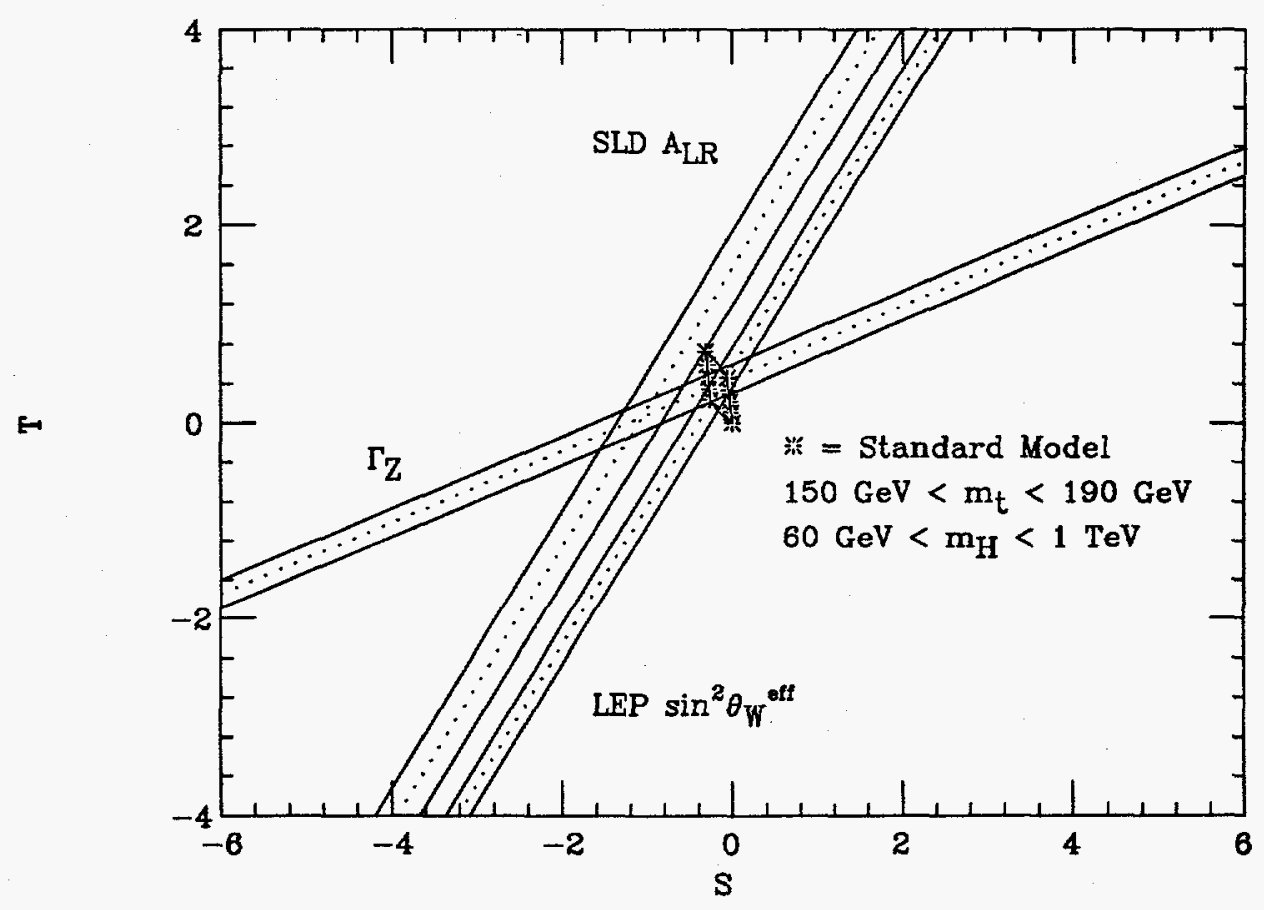

Figure 5-10: The projection of electroweak observables in S-T space. 
In this graph, the incompatibility of the SLD measurement with the LEP measurements and the Standard Model is most obvious. It is interesting to note that if the SLD value of $\sin ^{2} \theta_{W}^{\text {eff }}$ is correct, the measurement favors a region of negative $S$, which is theoretically challenging to explain. That is, it is difficult to create models which predict negative values of $S$. Supersymmetric and technicolor models, for instance, both predict positive $S$ [17].

Figure 5-11 shows the result of fitting the best values of $S, T$, and $\alpha_{\mathrm{S}}$ to 13 observables, including the SLD measurement of $A_{L R}$ [81]. Again, the Standard Model is encompassed by the parallelogram for the same range of top and Higgs masses given in figure 5-10. The fit results are

$$
\begin{gathered}
S=-0.35 \pm 0.23 \\
T=+0.24 \pm 0.22 \\
\alpha_{s}=0.124 \pm 0.005 \\
\chi^{2} / \text { d.o.f. }=14.8 / 10, \text { probability }=13.9 \%
\end{gathered}
$$

The overall fit is consistent with the Standard Model, as a result of the statistical power of the LEP measurements. However, we again see a slight but not terribly significant tendency towards regions of negative $S$.

\subsection{Future Experimental Precision}

SLD has been approved for four more years of polarized running, and with even higher electron polarization and increased luminosity, we hope to measure the effective Weinberg angle to a precision of 0.0003 . The projected error on $\sin ^{2} \theta_{W}^{\text {eff }}$ for and $80 \%$ polarization cathode and a systematic error of $1 \%$ is shown in figure 5-12. If the same central value of $A_{L R}$ reported in this document is measured in future running, the Standard Model will be in trouble, and electroweak physics will become even more interesting than it already is. 


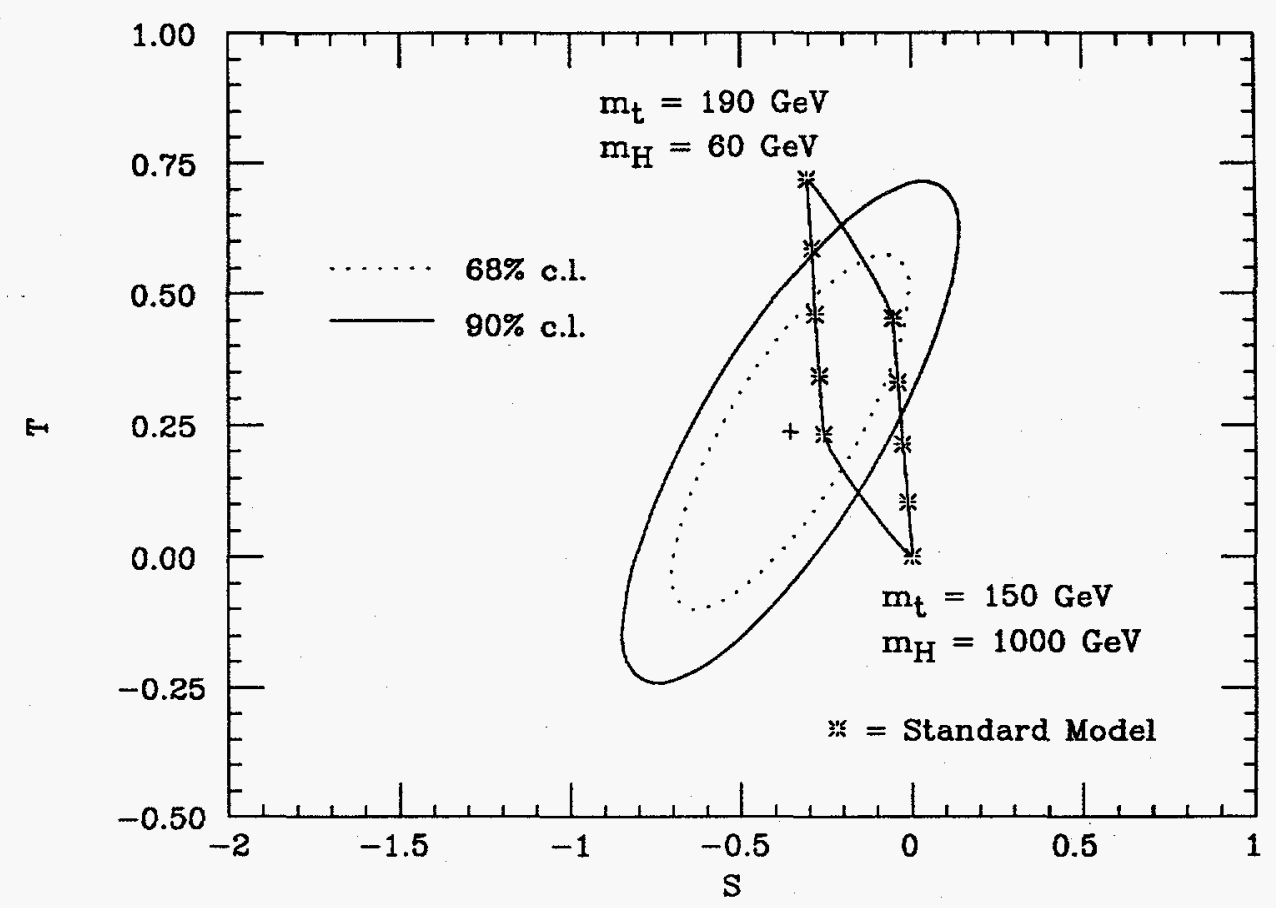

Figure 5-11: Standard Model fit to electroweak observables.

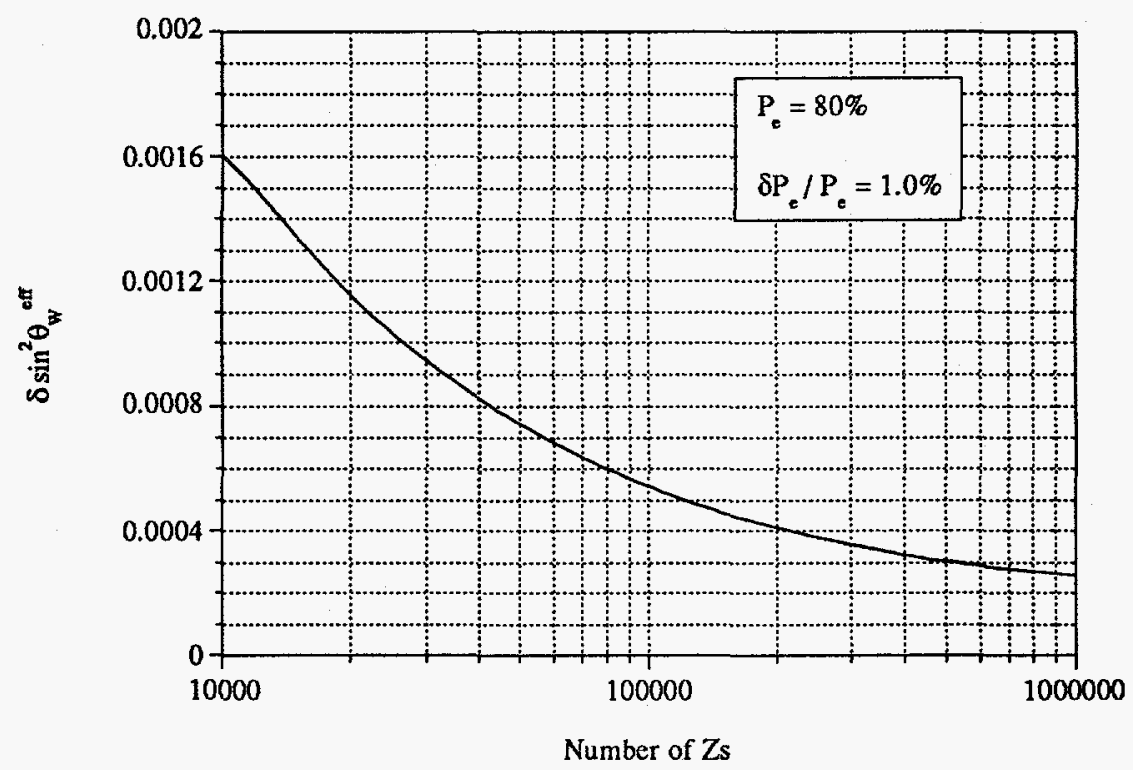

Figure 5-12: The projected error on the effective weak mixing angle. 


\section{References}

[1] A concise description of the 1993 experiment is found in:

K. Abe et al., Phys. Rev. Lett. 73, 25 (1994).

It must be noted that the analysis presented in this thesis differs in some details from that described in the citation.

[2] S. L. Glashow, Nucl. Phys. 22, 579 (1961).

[3] A. Salam and J. C. Ward, Phys. Lett. 13, 168 (1964);

A. Salam, Elementary Particle Theory: Relativistic Groups and Analyticity (8th Nobel Symposium), edited by N. Svartholm (Almqvist and Wiskell, Stockholm, 1968), p. 367.

[4] S. Weinberg, Phys. Rev. Lett. 19, 1264 (1967).

[5] We follow the notation and exposition of C. Quigg, Gauge Theories of Strong, Weak, and Electromagnetic Interactions, The Benjamin/Cummings Publishing Company, Inc., Reading, Massachusetts, 1983.

[6] P. W. Higgs, Phys. Rev. Lett. 12, 132 (1966); 13, 508 (1964);

P. W. Higgs, Phys. Rev. 145, 1156 (1966);

F. Englert and R. Brout, Phys. Rev. Lett. 13, 321 (1964);

G. S. Guralnik et al., Phys. Rev. Lett. 13, 585 (1964);

T. W. B. Kibble, Phys. Rev. 155, 1554 (1967). 
[7] Particle Data Group, Phys. Rev. D45, 11 (1992), Part II.

[8] B. Pietrzyk, LAPP-EXP-94.07, May 1994.

Summary of LEP results presented at the 1994 Moriond conference.

[9] F. Abe et al., FERMILAB-PUB-94-097-E, April 1994.

[10] A review of the properties of $A_{L R}$ can be found in:

D. C. Kennedy et al., Nucl. Phys. B321, 83 (1989).

[11] M. Swartz, SLAC-PUB-5219, March 1990.

[12] For an comprehensive discussion of the $Z$ lineshape and initial-state radiation, see M. E. Peskin, SLAC-PUB-5210, March 1990, and Proceedings of the 17th SLAC Summer Institute on Particle Physics, edited by E. C. Brennan, p. 71, (1990).

[13] E. A. Kuraev and V. S. Fadin, Sov. J. Nucl. Phys. 41, 466 (1985).

[14] G. Bonneau and F. Martin, Nucl. Phys. B27, 381 (1971).

[15] R. Cahn, Phys. Rev. D36, 2666 (1987).

[16] W. Marciano, Phys. Rev. D20, 274 (1979).

[17] M. E. Peskin and T. Takeuchi, Phys. Rev. D46, 381 (1992).

[18] D. C. Kennedy and B. W. Lynn, Nucl. Phys. B322, 1 (1989).

[19] B. W. Lynn and C. Verzegnassi, Nuovo Cim. 94A, 15 (1986).

[20] A. Sirlin, Phys. Rev. D22, 971 (1980).

[21] M. Demarteau et al., Combining W Mass Measurements, DONOTE 2115.

[22] B. Lynn, M. Peskin, and R. G. Stuart, Physics at LEP, Proceedings of the Workshop, Geneva, Switzerland, edited by J. Ellis and R. Peccei (CERN Report \# CERN 86-02, Geneva, 1986), Vol. 1. 
[23] M. Consoli and W. Hollik, $Z$ Physics at LEP 1 , Proceedings of the Workshop, Geneva, Switzerland, edited by G. Altarelli, R. Kleiss, and C. Verzegnassi (CERN Report \# CERN 89-08, Geneva, 1989), Vol. 1.

[24] D. Bardin et al., CERN-TH. 6443/92, May 1992. See also D. Bardin et al., Z. Phys. C44, 493 (1989).

[25] A. Olshevski, P. N. Ratoff, and P. B. Renton, CERN-PPE/93-88, May 1993.

[26] N. Phinney, Int. J. Mod. Phys. A, Proc. Suppl. 2A, 45 (1993).

[27] D. Schultz et al., SLAC-PUB-6275, June 1993.

[28] D. T. Pierce and F. Meier, Phys. Rev. B13, 5484 (1976).

[29] L. G. Levchuk, KHFTI-92-32, June 1992.

[30] R. E. Kirby et al., SLAC-PUB-6006, December 1992.

[31] M. Woods et al., SLAC-PUB-5894, September 1992.

[32] C. Adolphson, F.-J. Decker, and J. Seeman, Flat Beam Studies in the SLC Linac, Proceedings of the 1993 Part. Acc. Conf., Washington, D.C., 1993.

[33] C. Adolphson et al., SLAC-PUB-6118, May 1993.

[34] K. Pitts, Ph.D. Thesis, University of Oregon, SLAC Report 446, 1994.

[35] J. D. Jackson, Classical Electrodynamics, 2nd ed., J. Wiley and Sons Inc., New York, 1975, p. 558.

[36] T. Limberg, P. Emma, and R. Rossmanith, SLAC-PUB-6210, May 1993.

[37] F.-J. Decker et al., SLD Note \#238, January 1994. It must be explained that at the time this document was released, the final Compton polarimeter calibration was not yet available. All numbers in this thesis have been corrected for the final calibration, and differ slightly from those in the Note cited. 
[38] M. Swartz et al., SLAC-PUB-6467, June 1993.

Contains a complete treatment of SLAC Møller polarimetry.

[39] R. Elia, Ph.D. Thesis, Stanford University, SLAC Report 429, 1994.

[40] J. Kent et al., SLAC-PUB-4922, March 1989.

[41] G. Blaylock, SLD Physics Note \#22, June 1993, and private communication.

[42] The SLD Design Report, SLAC Report 273, 1984.

[43] C. J. S. Damerell et al., Nucl. Inst. and Meth. A328, 472 (1987).

[44] S. C. Berridge et al., IEEE Trans. Nucl. Sci. NS-39, 242 (1992).

[45] K. Abe et al., SLAC-PUB-6226, June 1993.

[46] P. N. Burrows et al., SLAC-PUB-5305, August 1990. See also

A. C. Benvenuti et al., SLAC-PUB-5332, November 1990;

D. C. Williams, Ph.D. Thesis, Massachusetts Institute of Technology, SLAC Report 445, 1994.

[47] D. Axen et al., Nucl. Inst. Meth. A328, 472 (1993).

[48] M. Swartz, SLAC-PUB-4656, June 1988, and Proceedings of the 15th SLAC Summer Institute on Particle Physics, edited by E. C. Brennan, p. 83, (1988).

[49] H. Veltman, Phys. Rev. D40, 2810 (1989).

[50] B. Schumm and R. Elia, SLD Note \#222, November 1992.

[51] B. Schumm, R. King, and E. Torrence, SLD Note \#241, March 1994.

[52] S. D. Drell, SLD Note \#226, September 1992.

[53] A. Lath and M. Woods, SLD Note \#236, June 1994.

[54] P. C. Rowson, M. Fero, M. Woods, and M. Zolotorev, SLD Note \#216 (revised), October 1992. 
[55] M. Woods, SLD Note \#223, October 1992.

[56] J. M. Yamartino, SLD Physics Note \#14, November 1992.

[57] S. González, SLD Physics Note \#24, October 1993.

[58] J. M. Yamartino, Ph.D. Thesis, Massachusetts Institute of Technology, SLAC Report 426, 1994. See Appendix A.

[59] K. T. Pitts, SLD Physics Note \#20, April 1993.

[60] H. Park et al., SLD Physics Note \#19, March 1993.

[61] R. Ben-David and H. Park, SLD Physics Note \#18, July 1993.

[62] R. Frey et al., SLD Note \#243, in progress.

[63] J. F. Ziegler, Nucl. Inst. and Meth. 191, 419 (1981).

[64] R. K. Adair and H. Kasha, "Cosmic-Ray Muons," Muon Physics, Volume 1: Electromagnetic Interactions, edited by V. W. Hughes and C. S. Wu, Academic Press (1977).

[65] H. Aihara et al., Z. Phys. C34, 1 (1987).

[66] F. E. Low, Phys. Rev. Lett., 14, 238 (1965).

[67] W. Beenakker, F. A. Berends, and S. C. van der Marck, Nucl. Phys. B349, 323 (1991).

[68] F. J. Decker, Luminosity Weighted Polarization Corrections, CN-399, 1994.

[69] B. Schumm, SLD Note \#234, October 1993.

[70] B. Schumm et al., SLD Note \#240, March 1994, and Paul Emma, private communication.

[71] P. Rowson, SLD Note \#244, in progress.

[72] A. A. Sokolov and I. M. Turnov, Dokl. Akad. Nauk. SSSR. 153, 1052 (1963). 
[73] M. Fero and D. Calloway, SLD Note \#233, August 1993.

[74] M. Fero et al., SLD Note \#232, August 1993.

[75] K. Yokoya and P. Chen, SLAC-PUB-4692, September 1988.

[76] T. Junk and T. Johnson, SLD Note \#227, November 1992.

[77] Tom Junk, private communication.

[78] P. C. Rowson, et al., SLD Note \#219, September 1992, and Peter Rowson, private communication.

[79] D. C. Kennedy et al., Z. Phys. C53, 617 (1992).

[80] K. Abe et al., Phys. Rev. Lett. 70, 2515 (1993).

The result reported in this citation:

$\sin ^{2} \theta_{W}^{\text {eff }}=0.2378 \pm 0.0056$ (stat.) \pm 0.0005 (syst.)

[81] The following measurements are included in the Standard Model fit:

$\sigma_{o}^{\text {had }}, \Gamma_{Z}, \mathrm{R}_{l}, A_{F B}^{\text {leptons }}, A_{F B}^{\text {b quarks }}, A_{F B}^{\text {c quarks }}, \mathrm{Q}_{F B}, A_{e}$ and $A_{\tau}$ (from $P_{\tau}$ ) [8];

$m_{W}[21], \mathrm{Q}_{W}[82], \mathrm{R}_{\mathrm{v}}[83], A_{L R}$ [this document].

[82] M. C. Noecker et al., Phys. Rev. Lett. 61, 310 (1988); [experiment]

S. A. Blundell et al., Phys. Rev. Lett. 65, 1411 (1990). [theory]

[83] G. Altarelli et al., CERN-TH. 6770/93. 